University of Louisville ThinkIR: The University of Louisville's Institutional Repository

Electronic Theses and Dissertations

$5-2019$

\title{
Polydrug use among people who use opioids, national survey on drug use and health 2002 to 2017.
}

Diana Kuo Stojda

University of Louisville

Follow this and additional works at: https://ir.library.louisville.edu/etd

Part of the Epidemiology Commons

\section{Recommended Citation}

Stojda, Diana Kuo, "Polydrug use among people who use opioids, national survey on drug use and health 2002 to 2017." (2019). Electronic Theses and Dissertations. Paper 3208.

https://doi.org/10.18297/etd/3208

This Doctoral Dissertation is brought to you for free and open access by ThinkIR: The University of Louisville's Institutional Repository. It has been accepted for inclusion in Electronic Theses and Dissertations by an authorized administrator of ThinkIR: The University of Louisville's Institutional Repository. This title appears here courtesy of the author, who has retained all other copyrights. For more information, please contact thinkir@louisville.edu. 
POLYDRUG USE AMONG PEOPLE WHO USE OPIOIDS, NATIONAL SURVEY ON DRUG USE AND HEALTH 2002 to 2017

\author{
By \\ Diana Kuo Stojda \\ BS, The University of North Carolina - Chapel Hill, 2012 \\ MPH, Boston University, 2007 \\ BS, University of Connecticut, 2005 \\ BA, University of Connecticut, 2005
}

\begin{abstract}
A Dissertation
Submitted to the Faculty of the

School of Public Health and Information Sciences

of the University of Louisville

in Partial Fulfillment of the Requirements

for the Degree of

Doctor of Philosophy in Public Health Sciences

Department of Epidemiology and Population Health

University of Louisville

Louisville, Kentucky
\end{abstract}

May 2019 
Copyright 2019 by Diana K. Stojda

All rights reserved 

POLYDRUG USE AMONG PEOPLE WHO USE OPIOIDS, NATIONAL SURVEY ON DRUG USE AND HEALTH 2002 to 2017

\author{
By \\ Diana Kuo Stojda \\ BS, The University of North Carolina - Chapel Hill, 2012 \\ MPH, Boston University, 2007 \\ BS, University of Connecticut, 2005 \\ BA, University of Connecticut, 2005
}

A Dissertation Approved on

April 15, 2019

by the following Dissertation Committee

Richard N. Baumgartner, Ph.D.

Martin T. Hall, Ph.D., MSSW

Nicholas Peiper, Ph.D., MPH

Kira C. Taylor, Ph.D., MS

Anne Baber Wallis, Ph.D., MHS 


\section{ACKNOWLEDGMENTS}

"No one is useless in this world who lightens the burdens of another."

\section{- Charles Dickens}

Those who know me well, know that I've been a student longer than I've been a working professional. The journey to obtaining my doctorate was a long and crazy one, with emotional ups and downs, but the one aspect of this journey that has helped me hold on to my sanity was the support of my family, friends, school colleagues, and everyone else who contributed in some way for me to realize my dream of lightening the burdens of others. To my parents, I thank you for supporting me emotionally (and financially!) in my academic pursuits; to my loving husband, Piotr, for putting up with the long distance and time apart; to my closest childhood friends, Christine Ku and Maria Shi, for their emotional comfort during times of deep stress; to all faculty and staff at the School of Public Health and Information Sciences who I have gotten to know throughout my time here; and to my partners in crime at UofL, Chisom Odoh and Colette Davis, for their friendship and emotional support while trying to finish the doctoral program. And finally, to my fantastic dissertation committee members: Drs. Richard Baumgartner, Martin Hall, Nicholas Peiper, Kira Taylor, and Anne Wallis, thank you all for giving me your time, energy, and expertise in making this dissertation a reality. 


\title{
ABSTRACT \\ POLYDRUG USE AMONG PEOPLE WHO USE OPIOIDS, NATIONAL SURVEY ON DRUG USE AND HEALTH 2002 to 2017
}

\author{
Diana K. Stojda
}

April 15, 2019

Introduction: Mortality due to overdose has been increasing since 2010 in the U.S., with an increase in the reported use of heroin and co-use of heroin and prescription opioids. Trends and correlates of polydrug use need to be analyzed to propose policies to reduce overdose risk. The overall objective of this study is to characterize high-risk polydrug groups among people who use opioids (PWUO) to understand the patterns of co-used substances and overdose risk factors.

Methods: Publicly available data from the 2002-2017 National Survey on Drug Use and Health were analyzed to obtain weighted prevalence rates and trends for PWUO groups, core substance use among each group, as well as polydrug latent classes. Trend analyses consisted of Rao-Scott Chi-square $\left(\chi^{2}\right)$ tests and joinpoint regression modeling. Multinomial logistic regression was used to determine associations between latent class membership and correlates such as sociodemographic factors, past year opioid use disorder (OUD) and residential transience.

Results: Overall prevalence of all individuals who reported the use of either a prescription pain reliever (PPR) or heroin in the past month has declined since 2006, however, the prevalence of the combination opioid use group has been increasing since 
2002. Multiple substance use by the past month combination opioid use group was substantially greater than those who exclusively used PPR or heroin in the past month, a finding that aligns with past research. Although it was hypothesized the combination opioid class would be characterized as having the highest risk of past year OUD, this was not the case; the Class with the highest adjusted odds of having past year OUD had the highest probability of reporting the past month use of heroin, marijuana, and other illicit substances [aOR $(95 \% \mathrm{CI}): 11.03(6.46,18.82)]$.

Conclusion: The increasing prevalence of polydrug use populations among people who use opioids, particularly those who have reported heroin use, underscores the need for prompt action from local, state, and federal stakeholders to pursue more aggressive action to stem the opioid crisis. Policies should conform to a unified public health framework, (e.g., Cascade of Care) and address both individual- and population-level strategies. 
TABLE OF CONTENTS

Page

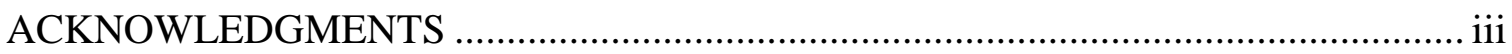

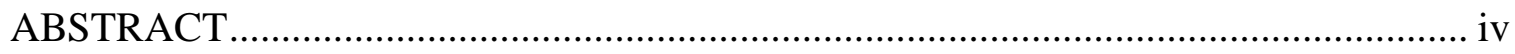

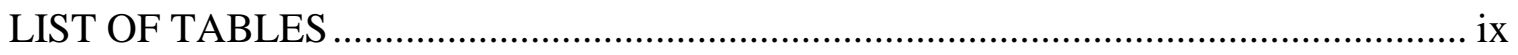

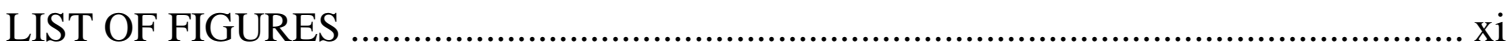

\section{CHAPTER I: INTRODUCTION AND STUDY OVERVIEW}

Historical Overview of Opioid Use in the United States ................................................1

Changing Trends of Prescription Opioid Misuse and Heroin Use in the United

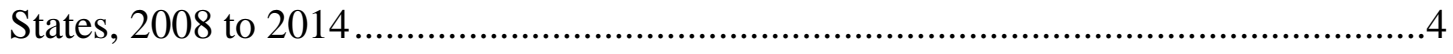

Defining Polydrug Use ……………………………………..................................

Rationale and Significance of Study ..................................................................6

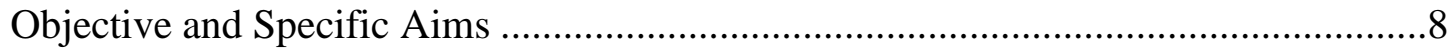

Hypotheses Testing for Aim 3 .....................................................................10

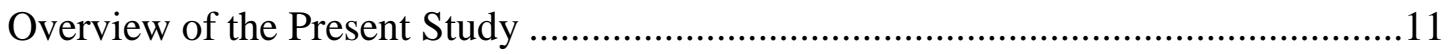

\section{CHAPTER II: LITERATURE REVIEW}

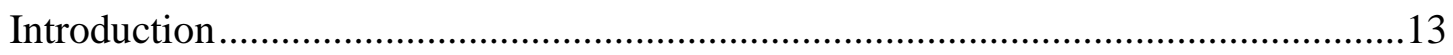

Opioid Literature from 1970 to $1990 \ldots \ldots \ldots \ldots \ldots \ldots \ldots \ldots \ldots \ldots \ldots \ldots \ldots \ldots \ldots \ldots \ldots \ldots \ldots \ldots \ldots \ldots \ldots \ldots \ldots \ldots \ldots \ldots \ldots . .13$

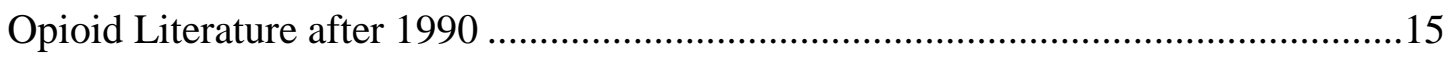


Epidemiology of People Who Use Opioids ...............................................................16

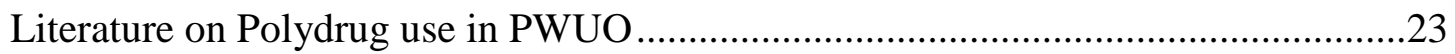

Drug, Set, and Setting Conceptual Framework........................................................2

Drug Factors: Commonly Co-used Substances in PWUO ........................................28

Set Factors: Individual Level Characteristics Associated with Opioid Misuse ............40

Setting Factors: Environmental Characteristics Associated with Opioid Misuse.........46

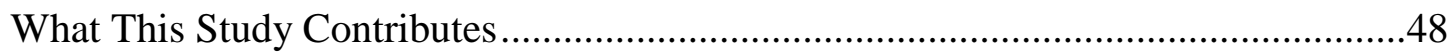

\section{CHAPTER III: RESEARCH DESIGN AND METHODOLOGY}

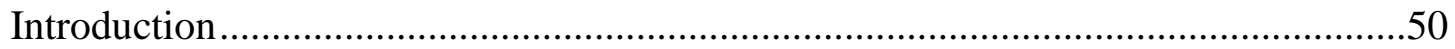

National Survey of Drug Use and Health (2002-2017) …….....................................50

The Current Study: Polydrug Use in PWUO, 2002-2017..........................................57

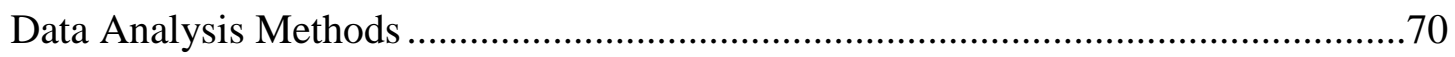

\section{CHAPTER IV: RESULTS}

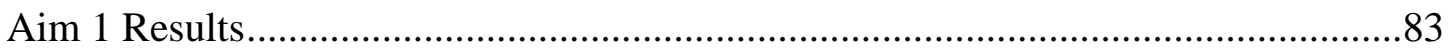

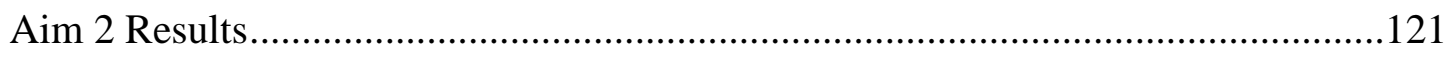

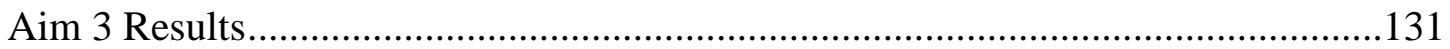

\section{CHAPTER V: DISCUSSION}

Longitudinal Prevalence of People Who Use Opioids and Their Substance

Use Patterns

Polydrug Latent Class Composition and Trends ...................................................153

Polydrug Latent Classes During Wave II of High Opioid Mortality and Associations with Set and Setting Correlates

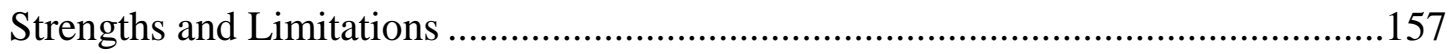

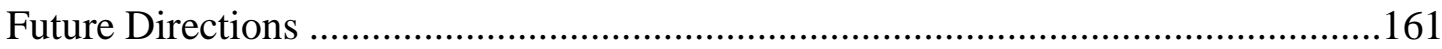




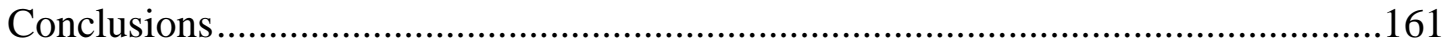

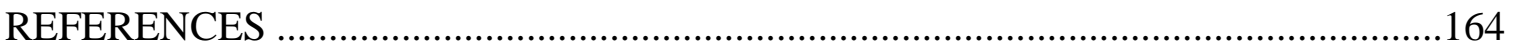

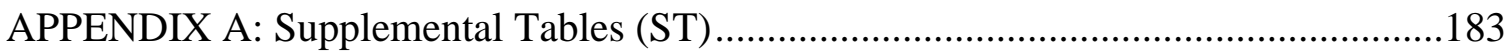

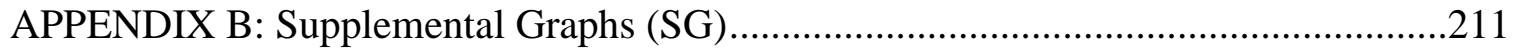

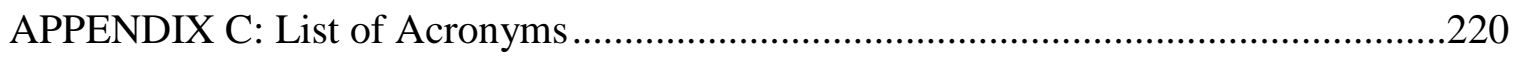

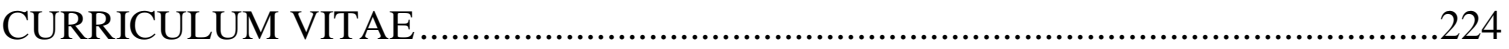




\section{LIST OF TABLES}

\section{Page}

2.1 Unadjusted prevalence estimates of past-year NMUPO by past-year drinking status for the overall sample.

2.2 Reported prevalence of lifetime hallucinogen and inhalant use among opioid use groups aged 18 years or older in $\operatorname{NESARC}(\mathrm{N}=9,140)$

2.3 Adjusted odds of NMUPO and SUD symptoms in early midlife as a function of medical use history with NMUPO during adolescence

3.1 Summary and Differences in Sampling Procedures in the NSDUH, 2002 to 2017 ....53

4.1 Weighted prevalence of all NSDUH participants by opioid use status and period ( $\mathrm{N}=621,571)$, NSDUH 2002 to 2017 .

4.2 Weighted prevalence of past month opioid use categories among PWUO by period ( $\mathrm{n}=16,985)$, NSDUH 2002 to 2017 ..

4.3 Rao-Scott $\chi^{2}$ analyses for the association of prevalence of each core substance and period for all PWUO $(\mathrm{n}=16,985)$, NSDUH 2002 to 2017

4.4 Rao-Scott $\chi^{2}$ analyses for the association of prevalence of each core substance and period for past month PPR-only use group $(n=15,897)$, NSDUH 2002 to $2017 \ldots .106$

4.5 Rao-Scott $\chi^{2}$ analyses for the association of prevalence of each core substance and period for past month heroin-only use group $(n=553)$, NSDUH 2002 to 2017

4.6 Rao-Scott $\chi^{2}$ analyses for the association of prevalence of each core substance and period for past month combination opioid use group $(\mathrm{n}=535)$, NSDUH 2002 to 2017

4.7 Overall size and weighted prevalence of the latent polydrug use subpopulations among PWUO ( $\mathrm{n}=16,985)$, NSDUH 2002 to 2017

4.8 Endorsement probabilities for each core substance by latent polydrug class ( $\mathrm{n}=16,985)$, NSDUH 2002 to 2017 
4.9 Weighted prevalence of polydrug latent classes in PWUO by period $(n=16,985)$, NSDUH 2002 to 2017.

4.10 Overall size and weighted prevalence of the polydrug latent classes among PWUO ( $\mathrm{n}=5,103)$, NSDUH 2010 to 2017.

4.11 Prevalence of set and setting correlates of polydrug latent classes $(\mathrm{n}=5,103)$, NSDUH 2010 to 2017

4.12 Rao-Scott $\chi^{2}$ analyses for the association of latent class membership and set and setting correlates among PWUO $(n=5,103)$, NSDUH 2010 to 2017.

4.13 Multivariable associations between latent class membership and sociodemographic factors $(n=5,103)$, NSDUH 2010 to 2017 .

4.14 Multivariable associations between latent class membership and age at prescription opioid initiation and past year OUD status $(n=5,103)$, NSDUH 2010 to 2017 146

4.15 Multivariable associations between latent class membership and county type and residential transience $(\mathrm{n}=5,103)$, NSDUH 2010 to 2017 


\section{LIST OF FIGURES}

Page

1.1 The Three Waves of the Rise in Opioid Overdose Deaths .............................................

1.2 Opioids Involved in United States Overdose Deaths, 2000 to 2016..............................3

2.1 Nonmedical Use of Past Year Heroin among Individuals Aged 26 and older, NSDUH 2016 to 2017.

2.2 Nonmedical Use of Past Year Prescription Pain Relievers among Individuals Aged 26 and Older, NSDUH 2016 to 2017 ................................................................20

2.3 Opioid-Related Overdose Deaths in the U.S. by State, 1999 to 2017 .........................22

2.4 Mortality Incidence Rate Ratios among Opioid Analgesic Users by

Benzodiazepine Use Status ............................................................................................30

2.5 Concurrent Opioid and Benzodiazepine Use by Prescription Attributes......................31

2.6 Heroin or synthetic opioid involved mortality rate trend from 2000 to 2015, National Vital Statistics System, United States

2.7 Drug Overdose Death Rates and Poverty Rates, United States 2016 ..........................47

3.1 Multistage Area Probability Sampling Procedure for the NSDUH …….....................52

3.2 NSDUH core substance indicators included in the latent class analysis model ...........75

3.3 Percentage of Times the Lowest Value Occurred in Each Class Model

for the AIC, CAIC, BIC, and Adjusted BIC

4.1 Weighted prevalence of people who reported past month use of opioids by period, $(\mathrm{n}=16,985)$, NSDUH 2002 to 2017.

4.2 Joinpoint analysis of the weighted prevalence of PWUO $(n=16,985)$

4.3 Weighted prevalence of all past-month opioid use categories among PWUO by period $(n=16,985)$, NSDUH 2002 to 2017 . 
4.4 Weighted prevalence of past-month heroin only and combination opioid groups among PWUO by period ( $\mathrm{n}=1,088)$, NSDUH 2002 to 2017 .

4.5 Joinpoint analysis of the weighted prevalence of past month PPR-only group $(\mathrm{n}=15.897)$

4.6 Joinpoint analysis of the weighted prevalence of past month heroin-only group $(\mathrm{n}=553)$.

4.7 Joinpoint analysis of the weighted prevalence of past month combination opioid Group $(\mathrm{n}=535)$

4.8 Overall weighted prevalence of past month core substance use among PWUO $(\mathrm{n}=16,985)$, NSDUH 2002 to 2017

4.9 Weighted prevalence of all levels of past month alcohol drinking among past month PWUO by period ( $\mathrm{n}=16,985)$, NSDUH 2002 to 2017

4.10 Weighted prevalence of reported past month cigarette smoking, other tobacco use, and marijuana use among past month PWUO by period ( $\mathrm{n}=16,985)$, NSDUH 2002 to 2017

4.11 Weighted prevalence of reported past month tranquilizer/sedative, cocaine, crack, hallucinogen/inhalant, and stimulant use among past month PWUO by period $(\mathrm{n}=16,985)$, NSDUH 2002 to 2017

4.12 Joinpoint analysis of the weighted prevalence of past month heavy alcohol use among all PWUO $(\mathrm{n}=16,985)$

4.13 Joinpoint analysis of the weighted prevalence of past month cigarette use among all PWUO $(\mathrm{n}=16,985)$

4.14 Joinpoint analysis of the weighted prevalence of past month other tobacco products use among all PWUO $(\mathrm{n}=16,985)$

4.15 Joinpoint analysis of the weighted prevalence of past month hallucinogen/inhalant use among all PWUO $(n=16,985)$

4.16 Joinpoint analysis of the weighted prevalence of past month stimulant use among all PWUO $(\mathrm{n}=16,985)$.....

4.17 Overall weighted prevalence of past month use of all core substances among the past month PPR-only group ( $\mathrm{n}=15,897)$, NSDUH 2002 to 2017 . 100

4.18 Overall weighted prevalence of past month use of all core substances among the past month heroin-only group $(\mathrm{n}=553)$, NSDUH 2002 to 2017 101 
4.19 Overall weighted prevalence of past month use of all core substances among the past month combination opioid group $(n=535)$, NSDUH 2002 to 2017

4.20 Weighted prevalence of all levels of past month alcohol drinking among past month PPR-only group by $(\mathrm{n}=15,897)$, NSDUH 2002 to 2017 .

4.21 Weighted prevalence of reported past month cigarette smoking, other tobacco use, and marijuana use among past month PPR-only group by period $(n=15,897)$, NSDUH 2002 to 2017

4.22 Weighted prevalence of reported past month tranquilizer/sedative, cocaine, crack, hallucinogen/inhalant, and stimulant use among past month PPR-only use group by period $(n=15,897)$, NSDUH 2002 to 2017

4.23 Joinpoint analysis of the weighted prevalence of past month heavy alcohol use among past month PPR-only group $(n=15,897)$

4.24 Joinpoint analysis of the weighted prevalence of past month cigarette use among past month PPR-only group $(\mathrm{n}=15,897)$

4.25 Joinpoint analysis of the weighted prevalence of past month other tobacco use among past month PPR-only group $(n=15,897)$

4.26 Joinpoint analysis of the weighted prevalence of past month hallucinogen/inhalant use among past month PPR-only group $(n=15,897)$.

4.27 Weighted prevalence of past month binge and heavy alcohol drinking among past month heroin-only group by period $(n=553)$, NSDUH 2002 to 2017 .

4.28 Weighted prevalence of reported past month cigarette smoking, other tobacco use, and marijuana use among past month heroin-only group by period $(n=553)$, NSDUH 2002 to 2017

4.29 Weighted prevalence of reported past month tranquilizer/sedative, cocaine, crack, hallucinogen/inhalant, and stimulant use among past month heroin-only group by period $(\mathrm{n}=553)$, NSDUH 2002 to 2017 .

4.30 Joinpoint analysis of the weighted prevalence of past month heavy alcohol use among past month heroin-only group $(\mathrm{n}=553)$

4.31 Joinpoint analysis of the weighted prevalence of past month cigarette use among past month heroin-only group $(\mathrm{n}=553)$.

4.32 Weighted prevalence of all levels of past month alcohol drinking among past month combination opioid group by period $(n=535)$, NSDUH 2002 to 2017 
4.33 Weighted prevalence of reported past month cigarette smoking, other tobacco use, and marijuana use among past month combination opioid group by period $(\mathrm{n}=535)$, NSDUH 2002 to 2017.

4.34 Weighted prevalence of reported past month tranquilizer/sedative, cocaine, crack, hallucinogen/inhalant, and stimulant use among past month combination opioid group by period $(\mathrm{n}=535)$, NSDUH 2002 to 2017

4.35 Joinpoint analysis of the weighted prevalence of past month crack use among past month combination opioid group $(n=535)$.

4.36 Joinpoint analysis of the weighted prevalence of past month hallucinogen/inhalant use among past month combination opioid group $(n=535)$

4.37 Joinpoint analysis of the weighted prevalence of past month stimulant use among past month combination opioid group $(n=535)$ 120

4.38 Information criteria comparisons for one to eight polydrug use classes in PWUO ( $\mathrm{n}=16,985)$, NSDUH 2002 to 2017.

4.39 Entropy comparisons for two to eight polydrug use classes in PWUO $(\mathrm{n}=16,985)$, NSDUH 2002 to 2017

4.40 Four Class Model Endorsement Probability Plot $(\mathrm{n}=16,985)$, NSDUH 2002 to 2017

4.41 Weighted prevalence of polydrug latent classes $(n=16,985)$, NSDUH 2002 to 2017

4.42 Joinpoint analysis of the weighted prevalence of Class $1(n=5,817)$.

4.43 Joinpoint analysis of the weighted prevalence of Class $2(n=8,709) \ldots$

4.44 Joinpoint analysis of the weighted prevalence of Class $3(n=553)$

4.45 Joinpoint analysis of the weighted prevalence of Class $4(n=1,906)$ 130 


\section{CHAPTER I}

\section{INTRODUCTION AND STUDY OVERVIEW}

\section{Historical Overview of Opioid Use in the United States}

Although pain-relieving substances, such as opioids, have been used by many civilizations throughout history, their use was relatively uncommon in the United States until the beginning of the American Revolution. ${ }^{1-4}$ Opioids used in the late $1800 \mathrm{~s}$ consisted predominantly of morphine and opium and were used widely for many reasons. ${ }^{5}$ For instance, mothers used opium tinctures to treat themselves and their children, soldiers used morphine to treat diarrhea and painful injuries, and Chinese immigrants imported opium to smoke in the U.S. ${ }^{5}$ Heroin was also administered iatrogenically, but because it was developed as a weaker pain reliever, it was not prescribed as liberally by medical doctors. ${ }^{3}$ Physicians also administered morphine to patients who suffered pain from conditions that were not well understood, such as infectious diseases, asthma, and gastrointestinal diseases. ${ }^{5}$ By the start of the $20^{\text {th }}$ century, opioids were being misused by the thousands. ${ }^{2}$ The rapid increase of people who used opium and heroin was eventually stemmed after the implementation of legislative policies (e.g., Harrison Act, 1909 Smoking Opium Exclusion Act) hindering the sale and import of these products, as well as discoveries by medical researchers (e.g., development of antibiotics and aspirin) which provided better treatment options. ${ }^{3,5}$ 
After the 1930s, heroin became the dominant opioid used in the U.S., which was likely the result of the rising cost of cocaine, as well as the effects of drug policies, like the Smoking Opium Exclusion Act. ${ }^{3}$ Also changing were the populations highest at risk of misusing opioids. Previously, Caucasian and Chinese populations were highest at risk, but after the 1950 s, the prevalence of heroin use increased among inner-city minorities. ${ }^{5}$ By the late 1980s, prescription opioid use began its slow ascent after Portenoy and Foley published results from their study that investigated the long-term safety of opioid pain relievers (OPR) in chronic non-cancer pain patients, concluding that OPRs may be used safely, a connection that was likely to be happenstance. ${ }^{6}$ However, a major factor in the steep use of OPRs came after 1995, when Purdue Pharma debuted a new product, OxyContin, and heavily campaigned the medical community for the next six years arguing for the importance of addressing pain symptoms among all patients. ${ }^{5}$

During the current opioid epidemic, it is estimated that more than 500,000 people died from drug overdoses from 2000 to 2015, with the majority involving an opioid. ${ }^{7}$ According to the National Center for Health Statistics, the number of overdose deaths involving opioids, including prescription opioids and heroin, has nearly quintupled from 1999 to $2016 .^{8}$ In the $21^{\text {st }}$ century, the opioid epidemic can be thought of as having three waves of high mortality, as shown in Figure 1.1. ${ }^{9}$ The first wave started in the 1990s and primarily involved prescription opioids, while the second wave of opioid mortality starting in 2010 involved mostly heroin overdoses. ${ }^{10}$ The third wave, characterized by a doubling of overdose death rates from 2013 to 2014, was related to synthetic opioids, including illicitly manufactured fentanyl and fentanyl analogs. ${ }^{10,11}$ And while mortality due to overdose has been increasing steadily since 2010 , the most recent data from the 
Centers for Disease Control and Prevention's (CDC) online database, the Wide-ranging ONline Data for Epidemiologic Research (WONDER), indicated that synthetic opioids have now surpassed all other categories of drugs involved in U.S. overdose death, as shown in Figure 1.2. ${ }^{12}$

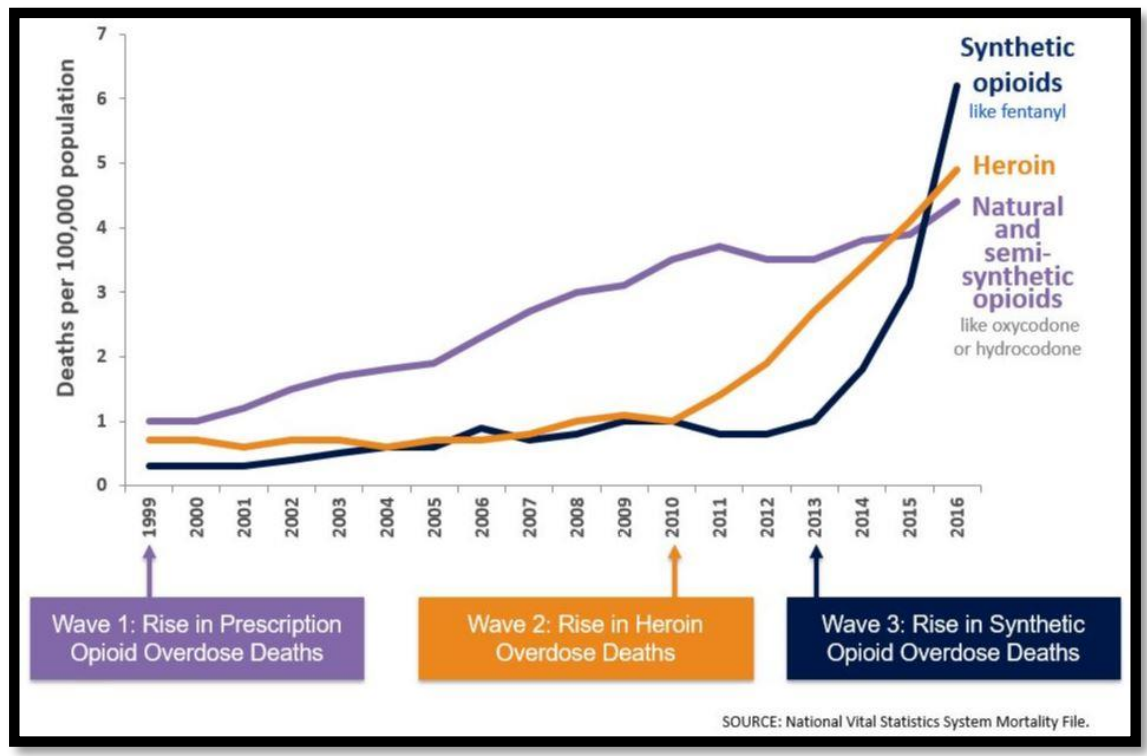

Figure 1.1 | The Three Waves of the Rise in Opioid Overdose Deaths

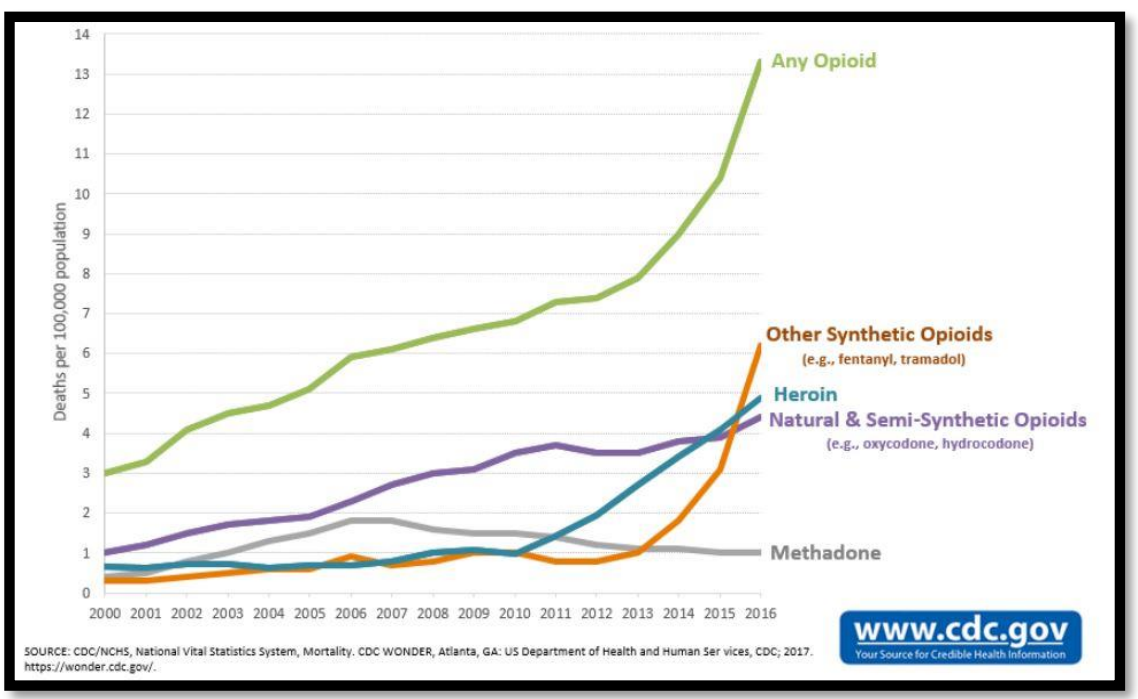

Figure 1.2 | Opioids Involved in United States Overdose Deaths, 2000 to $2016^{1}$

\footnotetext{
${ }^{1}$ Among the more than 64,000 drug overdose deaths estimated in 2016, the sharpest increase occurred among deaths related to fentanyl and fentanyl analogs (synthetic opioids) with over 20,000 overdose deaths. Source: CDC WONDER
} 
Based on nationwide data from health insurance claims, the total economic burden on the societal costs of prescription opioid use in the U.S. (including workplace, health care, and criminal justice costs) was estimated to be $\$ 55.7$ billion $^{2}$ per year from 1999 to $2007 .^{13}$ The workplace costs considered in this study were derived from loss of earnings due to premature death and reduced compensation or lost employment, while health care costs primarily reflected treatment costs. Finally, criminal justice costs included the cost of operating correctional facilities and employing police. ${ }^{13}$ However, the opioid epidemic has continued to progress. Based on the most current data from the National Vital Statistics System (NVSS) and the 2013 results of the National Survey on Drug Use and Health (NSDUH), it is now estimated that the total economic burden was $\$ 78.5$ billion in 2013. More than one-third of this cost is attributed to increased health care and substance abuse treatment costs, while approximately one quarter was borne by the public sector in health care, substance abuse treatment, and criminal justice costs. ${ }^{14}$

\section{Changing Trends of Prescription Opioid Misuse and Heroin Use in the United States, 2008 to 2014}

After the first wave of high opioid-related overdose deaths, there has been a noticeable downturn in the reported misuse of prescription opioids with a simultaneous increase in the use of heroin. ${ }^{15}$ In order to examine this relationship more closely, Cicero and colleagues collected data on a quarterly basis from January 1, 2008 through September 21, 2014 using self-administered surveys among independent cohorts with opioid dependence who were entering non-methadone maintenance programs

\footnotetext{
2 This cost was adjusted for inflation and represents the cost in United States dollars (USD) in 2009
} 
nationwide. ${ }^{16}$ The investigators found that rates of exclusive prescription opioid misuse decreased starting in 2010, while concurrent misuse of heroin and prescription opioids in the previous month increased by about $10.3 \%$ on average from 2008 (23.6\%) to 2014 $(41.8 \%) .{ }^{16}$ And although the use of heroin-only was low in the study population, the prevalence more than doubled during the study period. ${ }^{16}$ Also, while limited research has documented the polydrug use patterns and trends during the first wave of high opioid mortality (i.e., from 1999 to 2009), many subsequent studies indicated that multiple substance use was occurring prior to $2010 \cdot{ }^{17-20}$ Furthermore, it has been documented that those who co-use OPRs and heroin have a high burden of polydrug use compared to those who only use prescription opioids or heroin. ${ }^{21}$ This finding suggests that polydrug classes emerging in 2010 may have substance use characteristics that are affecting their increase in prevalence compared to those in Wave I of high opioid mortality in the United States.

\section{Defining Polydrug Use}

Because the main purpose of this study is to investigate polydrug use in PWUO, it is important to define the term. In 1975, the National Institute on Drug Abuse released a monograph titled "Operational Definitions in Socio-Behavioral Drug Use Research". ${ }^{22}$ While the monograph discussed a multitude of topics, such as the history of drug use, functional taxonomy of drugs, and conditions of drug use, it also included a chapter on defining "polydrug use". ${ }^{22}$ The chapter delineates the three different concepts listed below based on contemporaneous literature. ${ }^{22}$ 
(1) Any use of more than one mood-altering drug by an individual at any time in the past (i.e., ever use).

(2) Any use of more than one mood-altering drug during a delimited recent period (i.e., non-overlapping recent use).

(3) Any use of more than one mood-altering drug within an overlapping time frame (i.e., overlapping use).

It should also be noted that a fourth concept could be described, which would include polydrug use that (4) does not include the use of opiates. ${ }^{22}$

These concepts were later refined by substance use researchers to reflect concurrent versus simultaneous drug use. Concurrent polydrug use has been defined in the literature as using more than one drug in the same period (e.g., in the past year). ${ }^{23}$ On the other hand, simultaneous polydrug use refers to the co-ingestion of different drugs at the same intake event. ${ }^{23,24}$ However, it should be noted that the current study will only be able to address concurrent use of more than one substance in PWUO due to the nature of the NSDUH methodology.

\section{Rationale and Significance of the Study}

As this third wave of opioid-related mortality progresses, a major priority that public health professionals must address are the fundamental causes of opioid-related overdose, and to use this information to prevent adverse health outcomes related to polydrug use. While not a new concept in the literature, polydrug use in an adult population of people who use opioids (PWUO) has thus far been investigated and defined as non-opioid drug use (e.g., benzodiazepines, alcohol drinking, cigarettes, marijuana, 
and cocaine). ${ }^{25-27}$ Polydrug use patterns have been explored using a variety of analytic strategies, including the assessment of individual drug covariates or other substance use disorders among a certain subpopulations, ${ }^{28-30}$ using bivariate (yes/no) substance combinations, ${ }^{27}$ and composite scores. ${ }^{31,32}$ More recently, person-centered approaches such as latent class analysis (LCA) and latent profile analysis (LPA) have emerged in the literature. ${ }^{19,33-37}$ However, most of the studies that examine patterns of polydrug use limited the scope of the types of substances included (e.g., drinking behaviors, injection drugs, illicit drugs), with specific foci on different subpopulations, such as adolescents, people who inject drugs, chronic pain patients, etc.

Therefore, this study fills an important gap in the literature by examining the prevalence and trends of polydrug use in the U.S. among all PWUO using LCA. The advantage of using LCA over other non-person-centered approaches, is the ability to determine the substances that are most probabilistically associated with the co-use of opioids. Knowing the composition of each polydrug latent class will allow us to refine policies and interventions to decrease the overall prevalence of overdose risk.

Additionally, associations between correlates of interest in PWUO will be assessed using data that marks the beginning of the changing opioid overdose risk environment (i.e., 2010 to 2017), which will allow us to characterize the current subpopulations of PWUO.

The public health significance of examining the prevalence and correlates of polydrug use among PWUO includes understanding the latent classes of people who are more likely to have adverse health effects and also provide a basis for exploring multiple drug interactions from a pharmacological and physiological standpoint. Finally, the results of this study could act as a catalyst for restructuring national surveys such as the 
NSDUH to update their methodologies to allow the examination of simultaneous polydrug use or add additional substance use modules (e.g. fentanyl)..

Not only will this study utilize multiple NSDUH core substance categories in the analysis, it will also build upon prior knowledge related to polydrug use in PWUO by examining the longitudinal trends of specific polydrug combinations concurrently used by PWUO using joinpoint regression analysis. In the current literature, trends of polydrug use have been tracked using different statistical methods, including specifying linear time trends using a year variable in logistic regression models, calculating the difference in prevalence and percent change in prevalence for the period endpoints, and even using automated machine learning tools to employ iterative rounds of data gathering related to polydrug use using social media (e.g., Twitter). ${ }^{18,21,38,39}$ Limitations of the current studies that use the logistic regression method of trend analysis mainly center on the reliance of self-reported data, but also include the absence of using person-centered measures like latent classes. Additionally, the use of machine learning tools are hindered by selection bias and the background noise of communication on social platforms. A better understanding of the changes in trends of polydrug use will allow public health professions to track substances that are being reported by larger numbers of PWUO in order to inform proper intervention strategies.

\section{Objective and Specific Aims}

The overall objective of this study is to characterize high-risk polydrug groups in PWUO in the United States to understand the patterns of co-used substances and 
overdose risk factors. Therefore, three specific aims and their corresponding subaims are proposed to achieve this objective:

Specific Aim 1. To examine the prevalence of polydrug use among PWUO in a nationally representative sample in the U.S. over the period 2002-2017

1.1 To ascertain the prevalence of the all PWUO as well as opioid use groups (i.e., heroin only, prescription opioid only, and combination opioids) and determine whether the prevalence differ over time.

1.2 To ascertain the prevalence of all past month substance use in all PWUO and opioid use groups and determine whether the prevalence differ over time.

Specific Aim 2. To examine the prevalence of discrete latent classes of polydrug use in PWUO using data from the United States over the period 2002 to 2017.

2.1 To identify the latent class variable of polydrug use in PWUO using the 11 core substance indicators (pain relievers, heroin, alcohol, tranquilizers/sedatives, tobacco [cigarettes], other tobacco products, marijuana, cocaine, crack, hallucinogens/inhalants, and stimulants).

2.2 To determine whether the prevalence of each latent class has remained constant.

3. To examine factors related to substance use in the context of polydrug use in PWUO from 2010 to 2017. 
3.1 To determine the prevalence and bivariable associations of the following covariates using the latent class variable as an outcome: age, sex, race/ethnicity, total household income, education level, employment status, marital status, age at prescription pain reliever initiation, opioid use disorder, depression, health insurance status, county type, and residential transience.

3.2 To determine the multivariable associations of specific combinations of variables using the drug, set, and setting model of substance use behavior to predict membership of the polydrug latent class.

\section{Hypotheses Testing for Aim 3}

For Aim 3, three hypotheses and their related sub-hypotheses will be assessed:

Hypothesis 1: Polydrug latent classes will differ by sociodemographic characteristics. Sub-hypothesis 1.1: The differences between the associations will be driven by the types and number of substances used within each Class, with the 'combination opioid polydrug Class' more likely than other Classes to be male, non-Hispanic white, unemployed, younger at time of survey, uninsured, and having less education and less total household income.

Hypothesis 2: Polydrug latent classes will differ by their opioid use history and OUD status.

Sub-hypothesis 2.1: The differences between the associations will be driven by the types and number of substances used within each Class, with the 'combination 
opioid polydrug Class' more likely than other Classes to have initiated prescription opioid use at an early age and to have opioid use disorder in the past year.

Hypothesis 3: Polydrug latent classes will differ by their environment.

Sub-hypothesis 3.1: The differences between the associations will be driven by the types and number of substances used within each Class, with the "combination opioid polydrug Class' more likely than other Classes to reside in a large metro and to have moved at least twice in the past year.

\section{Overview of the Present Study}

To tackle these gaps in knowledge, publicly available data from 2002 to 2017 from the NSDUH was used to examine patterns of polydrug use in PWUO and to evaluate the effects of key covariates on high-risk polydrug use in that population. NSDUH is the premier surveillance system for monitoring drug use behavior because it is a cross-sectional survey administered every year by the Substance Abuse and Mental Health Services Administration (SAMHSA), in conjunction with the Research Triangle Institute (RTI). It utilizes a 50-state sampling design with an independent, multistage area probability sample for all states and the District of Columbia. ${ }^{40}$ Its primary purpose is to measure the prevalence and correlates of drug use among noninstitutionalized civilians in the United States. ${ }^{40}$ The participants recruited to complete the survey are aged 12 years and older, non-institutionalized, and English and Spanish speaking. ${ }^{40}$ Given the magnitude of topics covered by the survey, it is dataset to capture information 
relevant to polydrug use behavior, the covariates of interest, as well as sociodemographic characteristics. It was ultimately preferred over other publicly available datasets such as the National Comorbidity Survey (NCS) or the National Epidemiologic Survey on Alcohol and Related Conditions (NESARC) because of its ability to track reported substance use trends every year, without sacrificing data on covariates that were of most interest in this study. 


\section{CHAPTER II}

\section{LITERATURE REVIEW}

\section{Introduction}

There are three main goals of this literature review; the first is to summarize major themes that appear in opioid and polydrug use literature before and following the 1990s. The importance of discussing these studies is to set the historical context to the current opioid epidemic, which largely diverge into heroin and OPR studies. Second, recent studies related to populations at higher risk of opioid-related polydrug use will be presented to elucidate what is known about the co-use of opioids and other substances. Third, studies related to PWUO which evaluate covariates belonging to the drug, set, and setting framework will be described.

\section{Opioid Literature from 1970 to 1990}

Although literature on polydrug use has been around since the early 1970s, research related to opioids in this context did not begin to burgeon until $2012 .{ }^{41-43}$ Prior to 1990 , literature specific to polydrug use consisted of commentaries or editorials about drug addiction, treatment strategies, and patterns and distributions of drug use in different populations (e.g., adolescents, mothers, nationally representative populations). ${ }^{41-45}$ And while these themes are not exclusively confined to the 1970s, one unique aspect was that 
only one type of opioid was mentioned - namely, heroin. Heroin was of significant concern during this period, especially because of the "War on Drugs" sociopolitical sentiment of drug use in the $1970 \mathrm{~s} .{ }^{46}$ Populations most at risk of heroin and other polydrug use were predominantly African American, male, and between the ages of 21 to $25 .{ }^{45}$

It was not until the early 1980s that literature on the use of OPRs in humans began to surface, many of which focused on evaluating the efficacy of iatrogenically administered OPRs, such as morphine and pethidine. ${ }^{47,48}$ Other early studies focused on chronic pain patients because of the risk of their inappropriate or even excessive use of multiple pain medications. ${ }^{49-51}$ In 1979, Maruta et al. conducted a study that sought to characterize substance use in nonmalignant pain patients. One such commentary that was very influential in establishing the opioid prescribing practices that were common in the 1990s and 2000s came from two physicians (Porter and Jick) at the Boston University Medical Center. They noted that out of 11,882 patients who received one narcotic preparation, only four were documented to have developed an addiction, with no prior history of addiction. ${ }^{52}$ Therefore, they concluded that developing narcotic addiction was rare in patients with no prior history, despite the widespread use of narcotic drugs in the hospital. ${ }^{52}$ In 1982, Turner et al. completed a study that suggested that the overuse of pain medications in their study population led to many adverse social and physical consequences (e.g., higher hypochondriasis and hysteria scores, incurring higher costs per month on medication spending). ${ }^{50,51}$

Although the overall message from the results of Turner et al. echoed the sentiment that existed in the early 1900s regarding the dangers of pain medication use, 
this was quickly overturned by research conducted by pharmaceutical companies. ${ }^{51}$ In 1986, a study was published in Pain by two physicians who sought to weigh in on the controversial use of opioid analgesics in non-cancer patients. ${ }^{6}$ Their retrospective study involved reviewing case histories of a total of 38 patients from two separate sources: active and former patients who had previously been given non-opioid analgesics at the Pain Clinic of Memorial Sloan-Kettering Cancer Center. ${ }^{53}$ After reviewing all patient files, Portenoy and Foley found that $63 \%$ of the study population had notably enhanced comfort after the use of OPRs, the results of which aligned with other studies during the time. ${ }^{6,54-56}$ Not only did they conclude that pain medication is safe to administer on a long-term basis, but they also argued that 'addiction' differed from 'physical dependence,' arguing that 'addiction' should be called 'psychological dependence.'6

\section{Opioid Literature after 1990}

In the final decade of the $21^{\text {st }}$ century, before the onset of the first wave of high mortality in the current opioid epidemic, polydrug use in PWUO remained confined to patient populations and many studies focused on understanding biological effects of opioids and substance detoxification processes. ${ }^{57,58}$ After 1999, the distributions and patterns of opioid use were reassessed, and many studies no longer focused on heroin;

OPR misuse was now a hot topic in substance research. ${ }^{7,59-61}$ However, it was eventually revealed that a faction of individuals who misused OPRs would eventually begin to use heroin, which further complicated previous understanding regarding PWUO, because of the presupposition that the demographics of those who use heroin differed greatly from those who were prescribed pain medication. ${ }^{62,63}$ 
Of marked importance around the turn of the $21^{\text {st }}$ century was understanding the characteristics of individuals who used prescription opioids. ${ }^{64-66}$ These included special populations such as individuals with chronic infectious diseases (e.g., HIV, hepatitis), adolescents and young adults, and people with psychological conditions (e.g., depression, anxiety). ${ }^{34,67,68}$ Based on the literature search conducted, it was evident that researchers in this field were starting to move away from non-cancer chronic pain patients as a study population and expanding their research goals in other high-risk groups. Additionally, studies investigating the toxicological and pharmacologic effects of polydrug use in PWUO were more abundant, including the possible potentiating effects of benzodiazepines on opioids. ${ }^{69-72}$ Most recently, literature related to opioid misuse now included a multitude of case reports regarding the role of fentanyl in the massive increase of opioid-related overdose deaths in the United States and internationally. ${ }^{73,74}$

\section{Epidemiology of People Who Use Opioids}

\section{Sociodemographic characteristics}

In the United States, studies in PWUO have ranged from those who only use prescription opioids, to those who only use heroin, and finally, a combination of the two. This oversimplification may have been due to necessity, since 'complex' is an understatement for the characterization of affected and at-risk populations in this epidemic, partially due to the intertwining opioid use behaviors. ${ }^{75,76}$ It should also be noted that many studies and reports on the current opioid epidemic used mortality records or observational data, which may report different and contradicting information regarding populations most at risk. 
Many reports on the distribution and trends on opioid mortality rely heavily on surveillance data from sources like the CDC WONDER, which base their analyses and characterization on specific opioid categories (i.e., natural, semi-synthetic, fully synthetic). For example, a recent Morbidity and Mortality Weekly Report (MMWR) detailed an increase from 2014 to 2015 in natural and semi-synthetic opioid overdose deaths, with a significant increase in death in men of all ages, women between 25 and 44 years of age, and White non-Hispanic populations. ${ }^{7}$ Methadone overdose deaths differed slightly during this period in that both sexes of all ages, especially those over 65 years of age experienced increased death rates. ${ }^{7}$ However, beginning in 2015, deaths involving synthetic opioids (excluding methadone) and heroin were highest in men aged 25 to $44 .^{7}$ From 2015 to 2016, updated opioid mortality rates were reported in a 2018 MMWR, which showed increases among both men and women, as well as those greater than 15 years of age. ${ }^{77}$ Additionally, males aged 15 to 24 had the largest rate change in mortality rates, indicating that younger populations are now being affected by heroin and prescription opioid deaths. ${ }^{77}$

Other reports related to opioid use are generated from NSDUH data. Multiple SAMHSA reports predominantly focus on the single use of prescription opioids and heroin, with the most recent report providing some statistics on their combined use. ${ }^{78,79}$ According to the 2015 SAMHSA report, past month nonmedical use of pain relievers from 2002 to 2014 was on the decline for individuals 12 years and older in the most recent years. ${ }^{78}$ In contrast, reported heroin use from 2002 to 2014 was shown to increase slowly, and was reported by a higher percentage of young adults (aged 18 to 25), while the percentage of adolescents who reported its use remained at a lower, and steady trend 
from 2012 to $2014 .^{78}$ In the 2018 NSDUH report, the prevalence of individuals who use heroin remained steady in the same age groups from 2015 to $2017 .{ }^{79}$ Non-medical use of prescription pain relievers (PPR) was also discussed, but trend data was not presented. ${ }^{80}$

In terms of racial and ethnic distributions of the opioid epidemic, much of the research investigating racial differences focus on non-Hispanic Whites, non-Hispanic Blacks, and Hispanics, which may be due to low representation of other racial groups or the high proportion of non-Hispanic Whites who are affected in this epidemic. Morbidity due to prescription opioid overdose was previously understood as a White, non-Hispanic epidemic, driven by a multitude of factors which include (but are not limited to) structural racism and physician prescribing practices. ${ }^{15,81,82}$ Structural racism is generally defined as the "totality of ways in which societies foster [racial] discrimination, via mutually reinforcing [inequitable] systems...(e.g., in housing, education, employment, earnings, benefits, credit, media, health care, criminal justice, etc.) that in turn reinforce discriminatory beliefs, values, and distribution of resources". 83 The effects of structural racism has been shown to result in the vilification of Black and Brown opioid abuse and dependence, especially in the media, which ultimately drove different public responses and policy interventions (e.g., criminalization of substance use behaviors in non-White populations). ${ }^{84}$

However, based on a recent MMWR publication, racial and ethnic changes in either 'any opioid' or 'just prescription opioid' deaths have significantly increased in the non-Hispanic White, non-Hispanic Black, and Hispanic populations from 2015 to 2016, with non-Hispanic Blacks having the greatest percent rate change in heroin and 
prescription opioid mortality. ${ }^{77}$ This indicated a decrease in racial disparities in opioid use and mortality. ${ }^{77}$

Other significant sociodemographic indicators of substance use morbidity and mortality found in the literature are academic attainment and income. A recent study looking at the educational gradients in relation to drug overdose using data from the National Vital Statistics Survey (NVSS) and the National Death Index from 2002 to 2011 showed that individuals with less than high school education consistently had the highest death rates from drug overdose (per 100,000 persons) for each time period analyzed (i.e., 1992-1996, 1997-2001, etc.). ${ }^{85}$ It has also been shown that those with lower incomes, especially those on publicly funded health insurance policies, are more likely to have an opioid use disorder (OUD) and to misuse opioids when compared to the general U.S. population. ${ }^{86-89}$

\section{Geographic distribution of opioid-related morbidity and mortality}

Figures 2.1 and 2.2 show the geographic distribution of the number of reported heroin and nonmedical use of prescription opioids (NMUPO) among those aged 26 and older (i.e., the population with the highest risk of opioid overdose) using NSDUH data from 2016 to 2017 , respectively. ${ }^{90}$ Regions of the U.S. with the highest percentage levels of reported heroin use included the Midwest (Indiana, Ohio, West Virginia), the Northeast (Pennsylvania, Connecticut, New Hampshire, Maine), and the West (Washington, Nevada). ${ }^{90}$ 


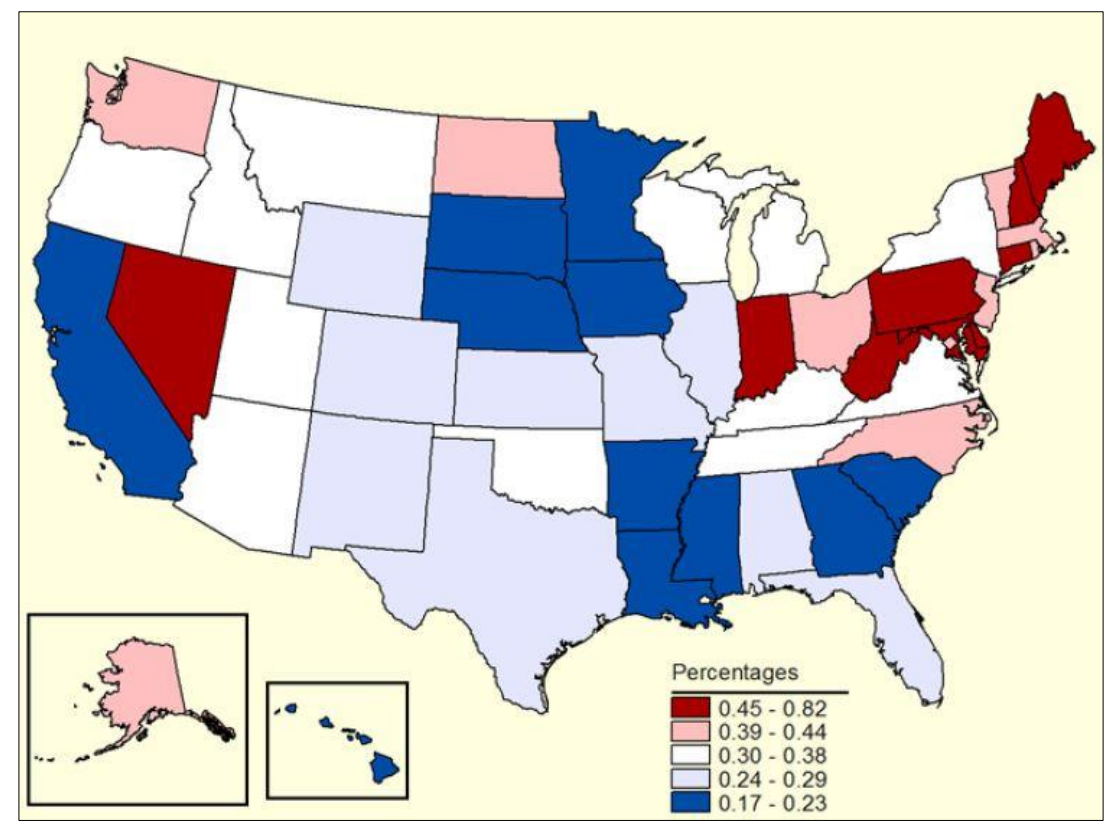

Figure 2.1 | Nonmedical Use of Past Year Heroin among Individuals Aged 26 and older, NSDUH 2016 to $2017^{90}$

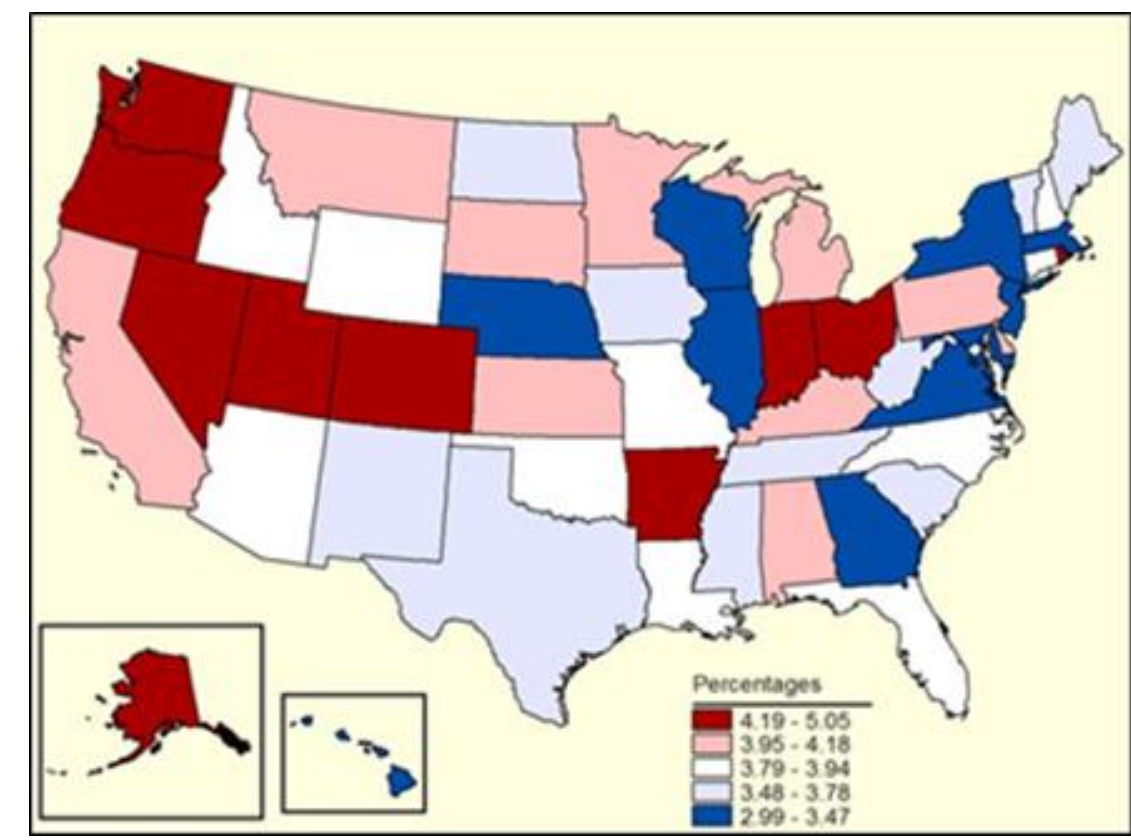

Figure 2.2 $\mid$ Nonmedical Use of Past Year Prescription Pain Relievers among Individuals Aged 26 and Older, NSDUH 2016 to $2017^{90}$

Figure 2.3 shows the opioid-related mortality rates using data from the 1999 to 2017 CDC WONDER as analyzed by the Kaiser Family Foundation. ${ }^{\S}$ Opioid deaths 
were defined using codes from the International Classification of Disease, $10^{\text {th }}$ Revision (ICD-10). ${ }^{\S}$ The ICD-10 codes included were opioids (T40.0, T40.1, T40.2, T40.3, T40.4, or T40.6); natural and semisynthetic opioids (T40.2); methadone (T40.3); synthetic opioids, other than methadone (T40.4); and heroin (T40.1). ${ }^{\S}$ As shown in Figure 2.3, states with the highest opioid-related mortality rates included Maine, New Hampshire, Ohio, and West Virginia. ${ }^{\S}$ 


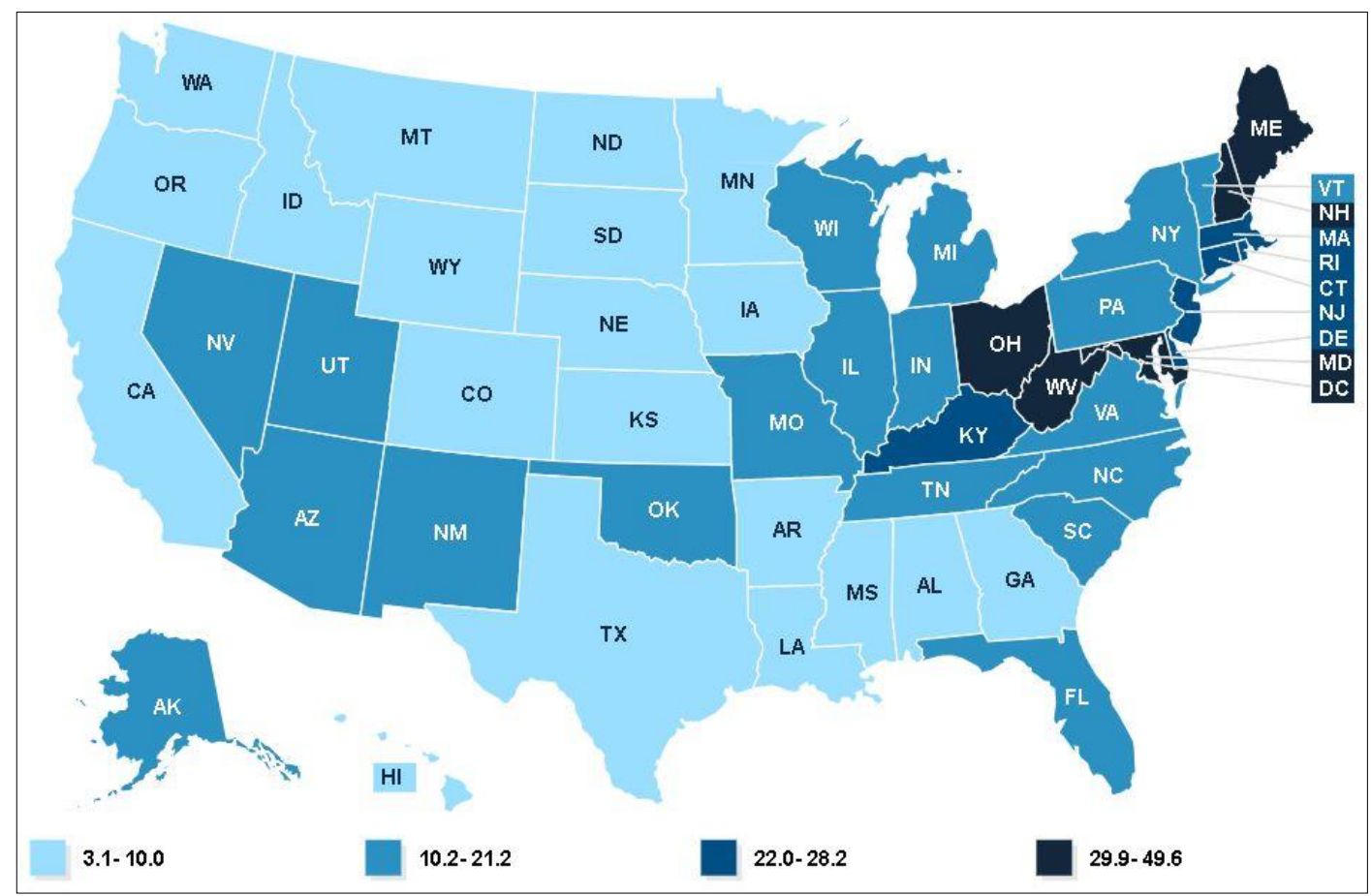

Figure 2.3 | Opioid-Related Overdose Deaths in the U.S. by State, 1999 to $2017^{\ddagger \S}$

\footnotetext{
The National Vital Statistics System multiple cause-of-death mortality files were used to identify drug overdose deaths. Drug overdose deaths were classified using the International Classification of Disease, Tenth Revision (ICD-10), based on the ICD-10 underlying cause-of-death codes X40-44 (unintentional), X60-64 (suicide), X85 (homicide), or Y10-Y14 (undetermined intent). Among the deaths with drug overdose as the underlying cause, the type of opioid involved is indicated by the following ICD-10 multiple cause-of-death codes: opioids (T40.0, T40.1, T40.2, T40.3, T40.4, or T40.6); natural and semisynthetic opioids (T40.2); methadone (T40.3); synthetic opioids, other than methadone (T40.4); and heroin (T40.1).
}

Age-adjusted death rates were calculated by applying age-specific death rates to the 2000 U.S. standard population age distribution. Death Rates are deaths per 100,000 population (ageadjusted).

Deaths from illegally-made fentanyl cannot be distinguished from pharmaceutical fentanyl in the data source. For this reason, deaths from both legally prescribed and illegally produced fentanyl are included in these data.

\section{${ }_{\text {Sources }}$}

Kaiser Family Foundation analysis of Centers for Disease Control and Prevention (CDC), National Center for Health Statistics. Multiple Cause of Death 1999-2017 on CDC WONDER Online Database, released 2018. Data are from the Multiple Cause of Death Files, 1999-2017, as compiled from data provided by the 57 vital statistics jurisdictions through the Vital Statistics Cooperative Program. Accessed at http://wonder.cdc.gov/mcd-icd10.html on January 10, 2019. 


\section{Literature on Polydrug use in PWUO}

Current polydrug use literature may be described as wide-ranging and includes a variety of populations (e.g., adolescents, young adults; HIV populations; those who use illicit substances; PWUO, people who inject drugs; etc.), but may not necessarily include opioids. ${ }^{91}$ However, because the focus of this study centers on PWUO, this section will review polydrug use studies primarily in the U.S. where participants have reported using either heroin or prescription opioids, with an emphasis on studies that use latent class analysis (LCA) to characterize these subpopulations.

\section{Opioid-related polydrug use in adolescents}

In a study by Katherine Keyes and colleagues, polydrug use was explored among young adults (aged 18-29) in the United States using data from the National Epidemiologic Survey on Alcohol and Related Conditions (NESARC) ${ }^{92}$ The results of their study found that $44 \%$ of people who currently use ecstasy reported taking at least three other classes of substances (i.e., cannabis, cocaine, opiates, tranquilizers), compared to only $1.6 \%$ in non-ecstasy users. ${ }^{92}$ Additionally, lifetime psychiatric comorbidities among current ecstasy users were much greater compared to non-illicit drug users. ${ }^{92}$

The idea that younger populations exhibited opioid-related polydrug use patterns was also validated several years later in a multi-site study by Lankenau et al. where the study population consisted of young adults (aged 16-25) who were known misusers of a prescription drug (e.g., opioids, tranquilizers, stimulants). ${ }^{93}$ Participants were asked questions within a multitude of key concepts including lifetime history of receiving a prescription medication, age at initiation of prescription drugs and other illicit drug use, 
lifetime history of using illicit drugs, and polydrug use with prescription drugs. ${ }^{93}$ Substances reported by participants as having been misused in the past 90 -days included a combination of opioids and tranquilizers, followed by stimulants (e.g., cocaine and amphetamines). ${ }^{93}$

\section{Polydrug use subpopulations among PWUO}

Nationally representative data has also been used to explore opioid-related polydrug groups. Wu et al. analyzed data from the 2001 and 2002 NESARC to determine whether there are subtypes of nonmedical opioid users and whether these subtypes may be characterized by distinct socioeconomic factors and other health-related variables. ${ }^{19}$ The target population of the NESARC is adults at least 18 years of age, and sampling for the survey involved a multistage cluster design. A total of 43,093 respondents were included in the analyses. ${ }^{19}$ Subtypes were determined using LCA techniques, and among this population, four LCA subtypes were identified: opioidmarijuana users (33\%), opioid-other prescription drug users (9\%), opioid-marijuanahallucinogen users (28\%), and opioid-polydrug users (30\%). ${ }^{19}$ Moreover, the prevalence of substance use disorders (SUD) by LCA-subtype were largely significant. ${ }^{19}$

A more recent study conducted by Bobashev et al. investigated patterns of polydrug use where participants were recruited from a syringe exchange program in Cleveland, Ohio. ${ }^{37}$ The overall purpose of the study was to establish the polydrug use patterns while distinguishing concurrent versus simultaneous polydrug use. ${ }^{37}$ The investigators also sought to characterize these subpopulations by their demographics and their attitudes regarding the different substances that they reported using. ${ }^{37}$ They found 
five polydrug group clusters using cluster analysis: 1) concomitant heroin, alcohol, and occasional crack use; 2) daily heroin and crack cocaine use; 3) mostly heroin; 4) daily heroin and marijuana use; and 5) part-time drug users. ${ }^{37}$ Bobashev et al. also found that polydrug use was facilitated by high availability and low cost of heroin and cocaine. ${ }^{37}$ Prescription opioid misuse and comorbid substance use were also explored using 2016 NSDUH data in a study published in early 2019 by Grigsby and Howard. ${ }^{94}$ The authors found that the majority of the participants reported prescription opioid and polydrug use $\mathrm{e}^{* *}$ [prevalence rate, PR $(95 \% \mathrm{CI}): 0.67 \%(0.56-0.79 \%)$, followed by: 1) prescription opioid only use; 2) prescription opioid and alcohol use; 3) prescription opioid and marijuana use; 4) prescription opioid and cigarette use; and 5) prescription opioid and hard drug use. ${ }^{94}$ Additionally, men, younger individuals, and those who reported an annual household income of less than $\$ 20,000$ per year were more likely to report past month prescription opioid misuse with an illicit drug or with polydrug use..$^{94}$

\section{Opioid-related polydrug use in people who inject drugs (PWID)}

Prescription opioid and heroin use has been documented in previous studies as precursors to other injection drug use. ${ }^{62,67,95,96}$ Furthermore, injection drug use (including opioids) is thought to increase a person's risk of contracting bloodborne infections that may lead to worse health outcomes. ${ }^{97,98}$ Using data from a longitudinal cohort study of PWID, Roth et al. examined distinct and habitual polydrug drug use (i.e., weekly or more frequent use) patterns among PWID living in San Diego, California using LCA to model the five substances that were reported to be most in use (i.e., heroin, methamphetamine,

\footnotetext{
** Grigsby and Howard defined polydrug use as past month misuse of prescription opioids and more than one recreational drug in the past month. ${ }^{73}$
} 
prescription drugs, alcohol, and marijuana) by different routes of administration (i.e., snorting, swallowing, injecting, and smoking). ${ }^{36}$

The LCA analysis conducted by Roth et al. identified two distinct classes of drug users: methamphetamine by multiple routes $(n=261 ; 51 \%)$ and heroin by injection $(n=$ $250 ; 49 \%) .{ }^{36}$ The conditional probabilities of prescription drugs and binge drinking were similar in both classes, but Class 1 members had a higher probability of marijuana use compared to Class 2 members (48.6\% versus $25.4 \%$, respectively). ${ }^{36}$ In their multivariable model, non-Hispanic Blacks [aOR (95\% CI): $3.23(1.53,6.84)]$ and Hispanics [aOR $(95 \% \mathrm{CI}): 1.84(1.14,3.01)]$ were more likely to belong in the primarily heroin injecting class, and those who reported ever overdosing on heroin, morphine, methadone, or oxycontin were more likely to belong in the primarily heroin injector class [aOR $(95 \% \mathrm{CI}): 1.89(1.23,2.89)] .^{36}$

In a study by Meacham et al., data from a prospective cohort study was used to identify substance use patterns among PWID in Tijuana. Four major criteria were used to decide which substance use variables went into the LCA model: type of substance, route of administration, co-injection, and frequency of use. ${ }^{33}$ From the LCA analysis, participants were classified into five subgroups ${ }^{33}$ :

1) Class 1 (polydrug and polyroute, plus cocaine; $5 \%$ of the sample)

2) Class 2 (polydrug and polyroute; $29 \%$ of the sample)

3) Class 3 (stimulant and heroin injection; 4\% of the sample)

4) Class 4 (methamphetamine and heroin injection, $10 \%$ of the sample)

5) Class 5 (predominantly heroin injection; $52 \%$ of the sample) 
Additionally, the investigators found that the two polydrug and polyroute classes (i.e., Classes 1 and 2) were more likely to report HIV risk behaviors (e.g., unprotected sex, having two or more sexual partners, drug use before or during sex, etc.) when compared to those who predominantly injected heroin (i.e., Class 5). Furthermore, Class 1

members were more likely than Class 5 members to report having had an overdose in the past six months [OR (p-value): $3.35(0.01)] .{ }^{33}$ And while the overall population from which the sample is drawn is not comparable to the U.S. population, the study results are consistent with the idea that those who have a greater amount of polydrug use behaviors are more likely to report needle sharing and overdose risk. ${ }^{99,100}$

\section{Drug, Set, and Setting Conceptual Framework}

A theoretical framework by Norman Zinberg, a professor in clinical psychiatry at Harvard Medical School will be used to guide the selection of correlates and to determine the analytical strategy. The drug, set, and setting model was used in one of his studies funded by The Drug Abuse Council (DAC), which sought to provide a description of participants who were categorized as controlled, marginal, and compulsive subjects based on preset conditions. ${ }^{101}$ The results of this study helped to pave the foundation of understanding opiate addiction. ${ }^{101}$

Drug in Zinberg's model refers to the type of substance used, how it is used (i.e., route of administration or whether other substances are combined during use), and the legal status of the substance. Set variables refer to the individual's unique traits (e.g., personality, preparation, intention, etc.) while setting factors may be broadly 
characterized as environmental components of an individual's life that influence the use or nonuse of substances (e.g., social, physical, and cultural environment). ${ }^{102}$

\section{Drug Factors: Commonly Co-used Substances in PWUO}

The purpose of the following subsections is to discuss research that has been conducted that explore the associations between opioid use and other substances that are surveyed in the NSDUH.

\section{Alcohol}

As with other medications, the effects of alcohol and opioid have been researched since the 1960s. ${ }^{103,104}$ The dangers of the co-use of these substances involve the significant respiratory distress that can occur due to the depressive effects of both substances on the central nervous system. ${ }^{105-107}$ The current extent of alcohol and opioid co-use has been documented in a study by McCabe et al. where data from the NESARC was analyzed to determine the prevalence of the misuse of four prescription drug classes, including prescription opioids. ${ }^{27}$ Of the 43,093 survey respondents, the unadjusted prevalence of past-year NMUPO by past-year drinking status for all participants is shown in Table 2.1. ${ }^{27}$ As shown in Table 2.2, the prevalence of individuals who misuse opioid analgesics across all categories of past-year drinking, was highest when compared to other prescription drug misuse categories. ${ }^{27}$ 
Table 2.1 | Unadjusted prevalence estimates of past-year NMUPO by past-year drinking status for the overall sample ${ }^{27}$

\begin{tabular}{|c|c|c|c|c|c|}
\hline $\begin{array}{c}\text { Past-year } \\
\text { drinking status }\end{array}$ & $\begin{array}{c}\text { Any } \\
\text { prescription } \\
\text { drug, \% } \\
\text { (SE) }\end{array}$ & $\begin{array}{c}\text { Opioid } \\
\text { analgesic, \% } \\
\text { (SE) }\end{array}$ & $\begin{array}{c}\text { Sedative, \% } \\
\text { (SE) }\end{array}$ & $\begin{array}{c}\text { Tranquilizer, } \\
\text { \% (SE) }\end{array}$ & $\begin{array}{c}\text { Stimulant, } \\
\text { \% (SE) }\end{array}$ \\
\hline Abstainer & $1.26(0.14)$ & $0.59(0.08)$ & $0.64(0.10)$ & $0.33(0.07)$ & $0.12(0.04)$ \\
\hline $\begin{array}{c}\text { Non-binge } \\
\text { drinking (no } \\
\text { AUDs) }\end{array}$ & $2.13(0.16)$ & $1.08(0.10)$ & $0.87(0.09)$ & $0.59(0.09)$ & $0.14(0.04)$ \\
\hline $\begin{array}{c}\text { Binge drinking } \\
\text { (no AUDs) }\end{array}$ & $4.01(0.30)$ & $2.23(0.24)$ & $1.27(0.15)$ & $1.19(0.17)$ & $0.70(0.12)$ \\
\hline $\begin{array}{c}\text { DSM-IV } \\
\text { alcohol abuse } \\
\text { only }\end{array}$ & $8.27(0.73)$ & $5.09(0.62)$ & $2.62(0.41)$ & $1.99(0.37)$ & $1.09(0.27)$ \\
\hline $\begin{array}{c}\text { DSM-IV } \\
\text { alcohol } \\
\text { dependence }\end{array}$ & $22.02(1.59)$ & $14.89(1.41)$ & $8.96(1.09)$ & $7.78(0.90)$ & $6.00(0.69)$ \\
\hline
\end{tabular}

\section{Benzodiazepines: Tranquilizers and Sedatives}

Benzodiazepines are a type of prescription drug commonly used to aid in relieving anxiety symptoms or insomnia and may be classified as either a tranquilizer or a sedative. ${ }^{108,109}$ When individuals take both substances, the effects include increased sedation, suppressed breathing, and impaired cognitive function, the interaction of which

may be additive or synergistic. ${ }^{108,110}$ Based on data from the IMS Health Vector One ${ }^{\circledR}$ Total Patient Tracker of patients from 2002 to 2014, the annual trend of concomitant prescribing of benzodiazepines and opioids rose from $6.8 \%$ to $9.6 \%$, which corresponded to a relative increase of $41 \% .^{111}$ The investigators also found that approximately half of all patients who were included in the study were prescribed both on the same day. ${ }^{111}$

The danger of benzodiazepine and opioid co-use was also assessed from a prospective observational cohort study in North Carolina. ${ }^{26}$ The study population 
consisted of all living North Carolina residents in $2010(\mathrm{n}=9,560,234) .{ }^{26}$ Data from the controlled substances prescription monitoring program was name-linked to mortality data from the North Carolina State Center for Health Statistics. ${ }^{26}$ The investigators found that $22.8 \%$ of all living residents in 2010 had been prescribed opioid analgesics; of these, $80 \%$ had also been prescribed benzodiazepines. ${ }^{26}$ The incidence rate ratios for overdose deaths involving opioid analgesics showed a dose-response relationship in all individuals, but especially in co-users of benzodiazepines, as shown in Figure 2.4. ${ }^{26}$ Overall, the rates of overdose death of residents who had been co-dispensed benzodiazepines and opioids were ten times higher than for opioid only users. ${ }^{26}$

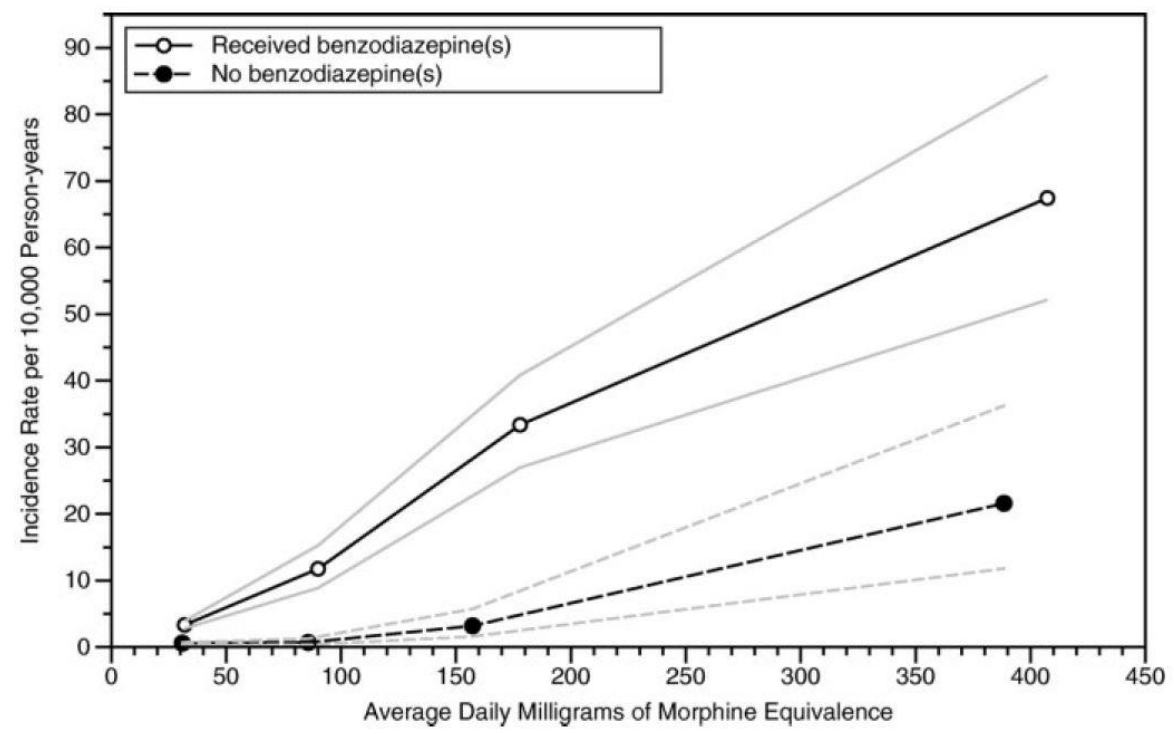

Figure 2.4 | Mortality Incidence Rate Ratios among Opioid Analgesic Users by Benzodiazepine Use Status ${ }^{26}$

Adding to this narrative are the results of a more recent investigation of the concurrent use of opioids and benzodiazepines by McClure et al. where specimens of patients who had been prescribed at least one type of prescription opioid or benzodiazepine, were reanalyzed for co-used substances. ${ }^{25}$ Nationwide patient samples 
collected from March 2015 to December 2015 were sent to Quest Diagnostics for cotesting of opioids and benzodiazepines using a combination of presumptive immunoassay screening and confirmatory quantitative mass spectrometry testing. ${ }^{25}$ Of the 231,228 sets of toxicologic test results available from 144,535 patients, 59,557 (25.8\%) were positive for both substances. ${ }^{25}$ Concurrent use was then broken down into subtypes based on prescription attributes, as shown in Figure 2.5. ${ }^{25}$ Based on the results of this study, the authors concluded that the extent of the co-usage of these substances goes beyond what was previously assessed using available prescription databases and monitoring programs because non-prescribed medications in the urine samples were detected. ${ }^{25}$

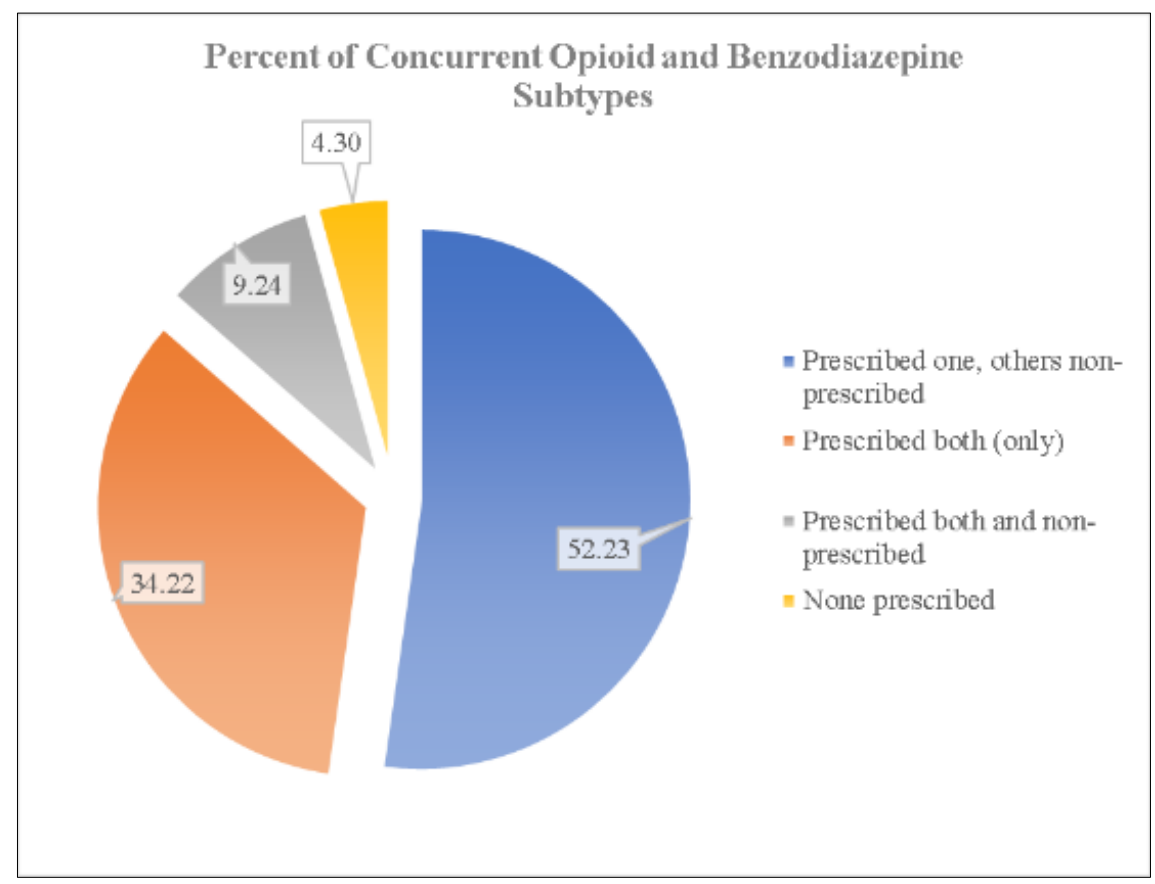

Figure 2.5 | Concurrent Opioid and Benzodiazepine Use by Prescription Attributes ${ }^{25}$ 


\section{Cigarette smoking and smokeless tobacco}

It has been shown in the literature that the connection between the co-use of cigarettes and opioids is a person's chronic pain condition, including mental health. ${ }^{112-115}$ Furthermore, major results from observational studies looking at the specific association between cigarette smoking and opioid use support the idea that smokers were more likely to report the use of opioids. ${ }^{116-118}$ Potential pathways for the continued co-use of cigarettes and opioids include pharmacological interaction between the two substances, or the mutual reinforcement of pain and tobacco use (i.e., individuals suffering from chronic pain conditions smoke cigarettes to alleviate pain). ${ }^{112,114,119}$ A study by Hooten et al. examined the association between depression and pain severity by smoking status as well as the association between depression and opioid use of the sample population $(\mathrm{N}=$ 1,241). ${ }^{120}$ The authors found that status as a current smoker was associated with the use of greater morphine dosages after adjusting for depression scores [beta $(\beta)$ coefficient (pvalue): 26.77 (0.003)]; this means that current smoking status was independently associated with a $27 \mathrm{mg} /$ day increase in morphine equivalent dose compared to never smokers. ${ }^{120}$

In a more recent study by John et al., data from the National Institute on Drug Abuse (NIDA) Clinical Trials Network (CTN) were analyzed to examine the extent of opioid misuse among different subgroups of tobacco users. ${ }^{121}$ The investigators found that approximately $14.0 \%$ of participants who reported past year tobacco use $(n=882)$, also reported past year opioid misuse, whereas the prevalence of past year opioid misuse was $2 \%$ among non-tobacco users. ${ }^{121}$ LCA was used to assess latent subgroups of tobacco users, and three classes were enumerated ${ }^{121}$ : 
1. Class 1 was referred to as the 'tobacco-minimal drug use group' and was characterized as having a low probability of any past year drug use. ${ }^{121}$

2. Class 2 was referred to as the 'tobacco-cannabis use group' and was characterized by a high probability of past year cannabis use with moderate probability of other past year drug use. ${ }^{121}$

3. Class 3 was referred to as the 'tobacco-opioid/polydrug use group' and was characterized by the high probability of opioid misuse, and moderate probability of past year cannabis, cocaine, and prescription stimulant and sedative use. ${ }^{121}$

When comparing the latent drug classes using sociodemographic factors, they found that Class 2 was more likely to be younger and have reported past year alcohol use. Additionally, Class 3 was more likely to be male, white, less educated, unemployed, disabled, and more likely to have reported past year alcohol use. ${ }^{121}$

With smokeless tobacco use, limited studies have been found that would be relevant in the context of opioids. However, in a 2014 article published by Fu et al., data from Wave I of the NESARC was analyzed to determine the association between snuff and chewing tobacco use and different psychiatric conditions, including OUD. ${ }^{122}$ The investigators found that those who used both chewing tobacco and snuff were almost twice as likely [aOR (95\% CI): $1.81(1.09,3.03)]$ ever to develop an OUD. ${ }^{122}$

\section{Crack and Cocaine}

Although these two substances are treated as separate categories in this study, freebase cocaine (or "crack") is a highly concentrated form of cocaine, that is typically 
smoked (whereas cocaine is snorted or injected). ${ }^{123}$ As such, both are derived from coca plants indigenous to the Andes Mountains in South America and induce short term effects such as extreme happiness and energy, irritability, or paranoia. ${ }^{123}$ The "high" effects of snorting or injecting the substance lasts for approximately 15 to 30 minutes. On the other hand, the effects of smoking may last only five to 10 minutes, exacerbating the urge to use crack. ${ }^{123}$

The biological effects of the co-use of cocaine and opioids (also known as "speedballing") differ from that of alcohol or benzodiazepines with opioids; instead of further depressing the CNS and leading to respiratory distress, it has been shown that both opioids and cocaine can act as powerful motivators of appetitive behavior. ${ }^{124-126}$ Furthermore, many individuals co-use heroin and cocaine to lessen the negative side effects of the individual substance. ${ }^{126}$ Additionally, studies have documented a variety of reasons for co-use that are seemingly unrelated to their biological effects, including the overall feeling of their co-use as being 'different' or 'better' than using a single substance alone, or as a method to self-medicate for mental health purposes or even reduce their intake of other substances (e.g., opioids). ${ }^{127-131}$ Macro-level factors related to availability have also been suggested in various news outlets. For instance, an analysis of data from the Drug Enforcement Administration's (DEA) System to Retrieve Information from Drug Evidence (STRIDE), showed that drug prices for cocaine have generally decreased from 1986 to 2012, as a result of organizational changes by drug traffickers. ${ }^{132}$

While fewer epidemiologic studies have focused on the co-use of crack or cocaine and opioids compared to alcohol or benzodiazepines, existing research has established that not only has heroin use increased among cocaine users, but that cocaine use disorder 
increased the risk of developing heroin and prescription opioid use disorder. ${ }^{18,88}$ From 2002 to 2013 , the prevalence rate (by 1,000 population) of reported past-year cocaine use among past-year heroin users increased by $87.1 \%$ when comparing 2002-2004 and 20112013 rates, and 34.0\% from 2008-2010 to 2011-2013 using NSDUH data. ${ }^{18}$ Moreover, individuals with cocaine use disorders in the U.S. had a 1.41 times greater prevalence of also having prescription opioid use disorders compared to those who did not [prevalence rate, $\mathrm{PR}(95 \% \mathrm{CI}): 14.3(13.74-14.97)] .^{88}$

To explore the relationship between concurrent opioid and cocaine use and its effect on mortality, McCall Jones et al. analyzed data from the NVSS to inform public health efforts how to curb overdose and to increase treatment strategies. ${ }^{17}$ Major findings of their study revealed a heterogeneous pattern of cocaine-related opioid overdose deaths. ${ }^{17}$ Cocaine-related deaths involving opioids increased from 2000 to 2006, and again from 2010 to 2015, as shown in Figure 2.6. ${ }^{17}$

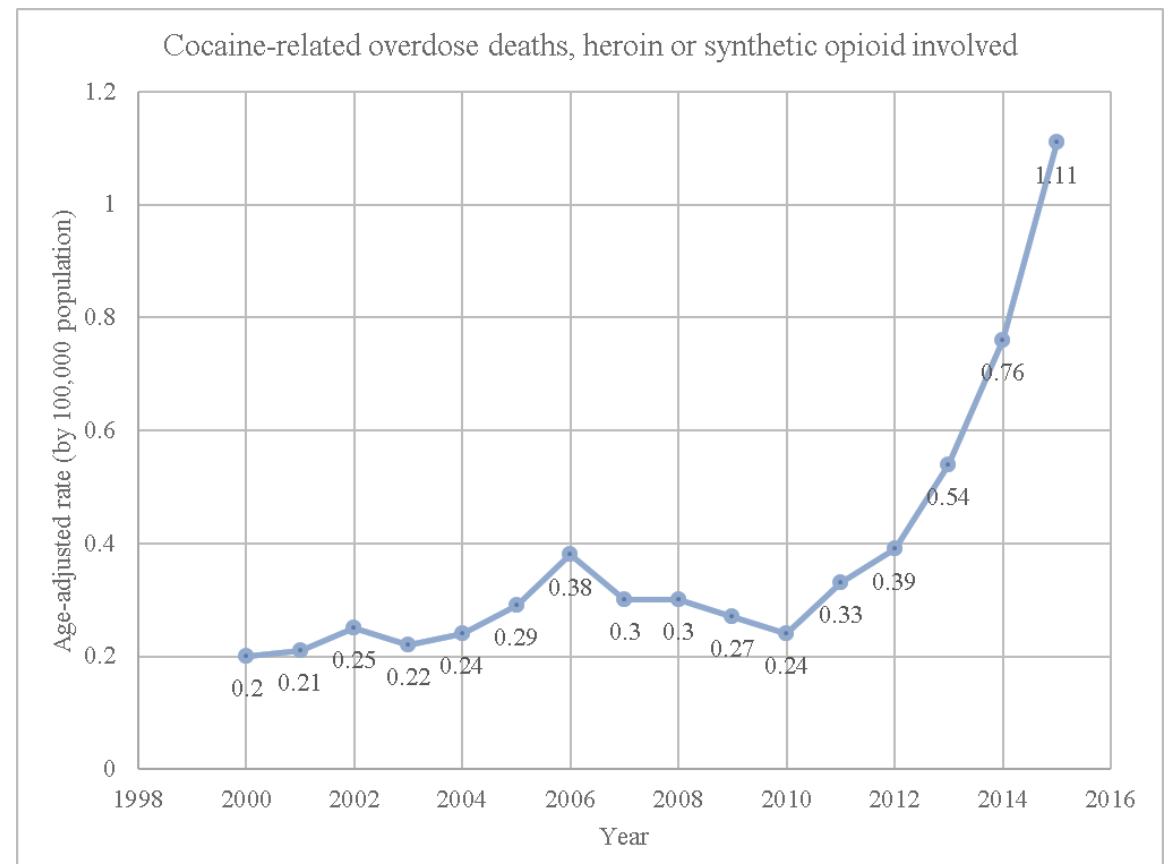

Figure 2.6 Heroin or synthetic opioid involved mortality rate trend from 2000 to 2015, National Vital Statistics System, United States ${ }^{17}$ 


\section{Hallucinogens and inhalants}

Hallucinogens (i.e., psychedelics) are substances that alter an individual's perception, thoughts, and feelings. ${ }^{133}$ In other words, they cause hallucinations or sensations that appear to be real. ${ }^{133}$ To date, some commonly used hallucinogens include ayahuasca, peyote, mescaline, and psilocybin. ${ }^{133}$ Inhalants are volatile substances that also have a psychoactive (i.e., mind-altering) effect when inhaled, and may be broadly categorized into four groups: volatile solvents, aerosols, gases, and nitrites. ${ }^{134}$

To date, the co-use of either hallucinogens or inhalants and opioids have been extremely limited, and somewhat mixed. In a 2011 study by Wu et al., data from the 2001 to 2002 NESARC was analyzed to determine whether opioid use groups (i.e., heroin-other opioid; other opioid only; heroin only; and non-opioid drugs users) differed in their patterns of drug use. ${ }^{135}$ Compared to non-opioid drug users, all other opioid use groups reported greater use of both hallucinogens and inhalants and this association was significantly different across all opioid use groups. ${ }^{135}$ Table 2.2 shows the prevalence of reported use for hallucinogens and inhalants in this study population. ${ }^{135}$

Table 2.2 Reported prevalence of lifetime hallucinogen and inhalant use among opioid use groups aged 18 years or older in $\operatorname{NESARC}(\mathrm{N}=9,140)^{135}$

\begin{tabular}{cccccccc}
\hline $\begin{array}{c}\text { Lifetime } \\
\text { substance } \\
\text { use }\end{array}$ & $\begin{array}{c}\text { Heroin- } \\
\text { other } \\
\text { opioids }\end{array}$ & $\begin{array}{c}\text { Other } \\
\text { opioids } \\
\text { only }\end{array}$ & $\begin{array}{c}\text { Heroin } \\
\text { only }\end{array}$ & $\begin{array}{c}\text { Non- } \\
\text { opioid } \\
\text { drugs }\end{array}$ & $\begin{array}{c}\boldsymbol{\chi}^{\mathbf{2}} \\
\text { (df) }\end{array}$ & $\begin{array}{c}\text { p- } \\
\text { value }\end{array}$ \\
\hline Hallucinogens & Yes & 97.4 & 46.1 & 68 & 19.2 & 128.5 & $<0.001$ \\
\cline { 2 - 7 } & No & 2.6 & 53.9 & 32.1 & 80.8 & $(3)$ & \\
\hline Inhalants & Yes & 46.3 & 18 & 24.1 & 4.4 & 87.3 & $<0.001$ \\
\cline { 2 - 7 } & No & 53.7 & 82 & 75.9 & 95.6 & $(3)$ & \\
\hline
\end{tabular}


In a more recent study by Pisano et al., the relationship between psychedelic use history and OUDs within an illicit opioid user population was analyzed using NSDUH data from 2008 to $2013 .{ }^{136}$ The authors found that psychedelic substance use was associated with a $27 \%$ reduced risk of past year opioid dependence [weighted $R R, R_{w}$ (95\% CI): $0.73(0.60-0.89)]$ among participants with a history of illicit opioid use. ${ }^{136}$

\section{Marijuana}

Another substance that has been recently assessed for its relationship with prescription opioid use disorder is marijuana. Initial literature, including news articles, has questioned whether the availability of marijuana may curtail the need for the use of prescription opioids. ${ }^{137-142}$ In a study by Bachhuber et al., a time-series ecologic analysis was conducted using state-level death certificate data in the U.S. from 1999 to $2010 .{ }^{140}$ The investigators found that the existence of medical cannabis laws (MCL) was significantly associated with lower state-level opioid overdose mortality rates. ${ }^{140}$ Also, in a recent study by Vigil et al., data from a preliminary study on a cohort of chronic pain patients with a history of habitual opioid use $(\mathrm{n}=37)$ were analyzed to establish whether there was an association between enrollment in the New Mexico Medical Cannabis Program (MCP) and opioid prescription use. ${ }^{142}$ The patients were enrolled in the MCP from April 1, 2010 to October 5, 2015 (i.e., 21 months), and it was determined that MCP enrollment was associated with higher age- and gender-adjusted odds of ceasing opioid prescriptions (95\% CI 1.89 to $157.36, \mathrm{p}=0.012)$ and reducing daily prescriptions opioid dosages (95\% CI 1.56 to $16.88, \mathrm{p}=0.007) .{ }^{142}$ 
Since these early studies and commentaries, research has been conducted to determine whether marijuana use has an effect on NMUPO; in a study published in 2018, Olfson et al. analyzed data from Waves I and II of the NESARC to evaluate whether marijuana use is associated with a change in the risk of incident NMUPO. ${ }^{143}$ The results of the prospective association analyses between cannabis use and NMUPO showed that those who reported cannabis use in Wave I had significantly increased odds of incident NMUPO in Wave 2, even after adjusting for demographics and clinical characteristics (e.g., other SUDs, depression, family history of drug use disorders) [aOR (95\% CI): 2.62 (1.86-3.69)]. ${ }^{143}$ The results of this study may be explained by the idea that cannabis use could lead to behavioral disinhibition, possibly increasing the risk of using other substances, including opioids. ${ }^{144}$

\section{Prescription stimulants}

Prescription stimulants are drugs that are generally provided to individuals with attention-deficit hyperactivity disorder (ADHD) and narcolepsy because they function to increase alertness, attention, and energy. ${ }^{145}$ Commonly prescribed stimulants include dextroamphetamine (Dexedrine $\left.{ }^{\circledR}\right)$, dextroamphetamine/amphetamine combination product (Adderall $®$ ), and methylphenidate (Ritalin ${ }^{\circledR}$, Concerta $\left.{ }^{\circledR}\right) .{ }^{145}$ Although observational studies on the co-use of opioids and prescription stimulants were extremely limited, a few studies have surfaced in recent years which focus on higher risk populations, such as those with ADHD as well as working adults. ${ }^{146,147}$

In a recent study by Wei et al., an analysis of 66,406 Medicaid-enrolled adults (aged 20 to 64) with ADHD was conducted using cross-sectional data from 1999 to 
2010. ${ }^{146}$ Their goal was to assess the prevalence and secular trends of concurrent stimulant and opioid use, as well as the factors associated with long-term use. ${ }^{146}$ It was determined that of the 21,723 adults with ADHD (32.7\% of total eligible patients), 5,004 (23.0\%) received prescription stimulants and opioids for a short time period (1-29 days), and 3,560 (16.5\%) received both drugs for at least 30 days (i.e., long term). ${ }^{146}$ Also, those who were prescribed stimulants were more likely to have been prescribed opioids for long term use compared to those who were not prescribed stimulants. ${ }^{146}$ Furthermore, the investigators found a significant increase in the prevalence of long-term concurrent stimulant-opioid use from 1999 to 2010 [adjusted prevalence relative ratio (95\% CI):

\section{$1.12(1.10-1.14)] .^{146}$}

Another recent study published by Perlmutter et al. investigated whether employment status was associated with NMUPO and nonmedical use of prescription stimulants (NMUPS). ${ }^{148}$ The study included adults aged 26 and older who participated in the 2011 to 2013 NSDUH and examined covariates that related to their employment status (i.e., employed full-time, employed part-time, unemployed, and not in the workforce) and past year NMUPO and NMUPS use. ${ }^{148}$ They found that the overall past year prevalence of NMUPO and NMUPS were 3.48 and $0.72 \%$, respectively; among those unemployed, the prevalence of NMUPO and NMUPS were the highest of the four employment groups, at 6.91 and $7.01 \%$, respectively. ${ }^{148}$ The researchers also determined that unemployed respondents had the highest odds of NMUPO [aOR (95\% CI): 1.45 (1.15-1.82)], while those not in the workforce had the highest odds of NMUPS [aOR (95\% CI): $1.71(1.22-2.37)]{ }^{148}$ 


\section{Set Factors: Individual Level Characteristics Associated with Opioid Misuse Age at prescription opioid initiation}

In recent years, the number of prescription opioid initiates in the adolescent population has prompted researchers to investigate whether age at initiation affects other substance use behaviors. In a previously described study, Lankenau et al. found that the age of initiation of stimulants, heroin, and prescription opioids may serve as a pathway to the misuse of other, especially illicit, substance use. ${ }^{93}$ Additionally, Cerda et al. analyzed data from the 2004 to 2011 NSDUH and developed discrete-time hazard models to estimate the age-specific hazards of heroin initiation if the respondent had a prior history of prescription opioid use in adolescents and young adults. ${ }^{63}$ Overall, it was revealed that heroin initiation was strongly affected by a prior history of prescription opioid misuse [hazard ratio $(95 \% \mathrm{CI}): 13.12(0.73,16.04)]$, however, when stratifying the results by age, those who were between the ages of 10 and 12 had the highest risk of transitioning to heroin use, regardless of race/ethnicity or income [hazard ratio (95\% CI): 1.84 (1.13, 2.99)]..$^{63}$

Stronger evidence that suggested the significance of 'age at initiation' comes from the analyses of data drawn from a prospective cohort study called Monitoring the Future (MTF). ${ }^{149}$ McCabe et al. examined the relationships among various factors such as NMUPO frequencies, NMUPO motives, and initiation of medical prescription opioid use and NMUPO during adolescence in relation to substance use disorders (SUDs) at age 35. ${ }^{149}$ From the descriptive analysis, the prevalence rates of SUD symptoms at age 35 were the highest for adolescents who either: 1) reported the medical use of prescription opioids after initiating NMUPO or 2) reported only NMUPO. ${ }^{149}$ Furthermore, 
adolescents in these two groups had significantly higher odds of alcohol use disorder (AUD), cannabis use disorder (CUD), and the other drug use disorders (ODUD) compared to adolescents who had no history of medical or nonmedical use of prescription opioids (refer to Table 2.3 for all adjusted odds ratios). ${ }^{149}$

Table 2.3 $\mid$ Adjusted odds of NMUPO and SUD symptoms in early midlife as a function of medical use history with NMUPO during adolescence

\begin{tabular}{|c|c|c|c|c|c|}
\hline \multicolumn{2}{|c|}{$\begin{array}{l}\text { Baseline characteristics } \\
\text { (modal age 18) }\end{array}$} & $\begin{array}{l}\text { Alcohol use } \\
\text { disorder } \\
\text { (AUD) } \\
\text { symptoms at } \\
\text { age 35; aOR } \\
(95 \% \mathrm{CI})^{\mathrm{a}}\end{array}$ & $\begin{array}{l}\text { Cannabis use } \\
\text { disorder (CUS) } \\
\text { symptoms at } \\
\text { age } 35 ; \text { aOR } \\
(95 \% \mathrm{CI})^{\mathrm{a}}\end{array}$ & $\begin{array}{l}\text { Other drug use } \\
\text { disorder } \\
\text { (ODUD) } \\
\text { symptoms at } \\
\text { age 35; aOR } \\
(95 \% \mathrm{CI})^{\mathrm{a}}\end{array}$ & $\begin{array}{l}\text { Any substance use } \\
\text { disorder (SUD) } \\
\text { symptoms at age } \\
35 ; \text { aOR }(95 \% \\
\text { CI) }\end{array}$ \\
\hline \multirow{5}{*}{$\begin{array}{c}\text { Lifetime } \\
\text { medical } \\
\text { use and } \\
\text { NMUPO }\end{array}$} & $\begin{array}{l}\text { No medical or } \\
\text { nonmedical use } \\
\quad(\mathrm{n}=6167)\end{array}$ & \multicolumn{4}{|c|}{ Reference } \\
\hline & $\begin{array}{l}\text { Medical use only } \\
\quad(\mathrm{n}=967)\end{array}$ & $\begin{array}{c}1.06 \\
(0.852,1.33)\end{array}$ & $\begin{array}{c}1.11 \\
(0.712,1.72)\end{array}$ & $\begin{array}{c}1.31 \\
(0.758,2.25)\end{array}$ & $\begin{array}{c}1.12 \\
(0.895,1.39)\end{array}$ \\
\hline & $\begin{array}{c}\text { Medical use } \\
\text { before } \\
\text { nonmedical use } \\
(\mathrm{n}=334)\end{array}$ & $\begin{array}{c}1.19 \\
(0.834,1.70)\end{array}$ & $\begin{array}{c}0.796 \\
(0.463,1.37)\end{array}$ & $\begin{array}{c}1.52 \\
(0.777,2.97)\end{array}$ & $\begin{array}{c}1.18 \\
(0.821,1.72)\end{array}$ \\
\hline & $\begin{array}{c}\text { Medical use } \\
\text { after nonmedical } \\
\text { use } \\
(\mathrm{n}=112)\end{array}$ & $\begin{array}{c}1.62 \\
(1.02,2.56)^{*}\end{array}$ & $\begin{array}{c}3.24 \\
(1.58,6.62)^{* *}\end{array}$ & $\begin{array}{c}7.22 \\
(3.75,13.9)^{* *}\end{array}$ & $\begin{array}{c}2.35 \\
(1.46,3.77)^{* *}\end{array}$ \\
\hline & $\begin{array}{c}\text { Nonmedical use } \\
\text { only } \\
(\mathrm{n}=259)\end{array}$ & $\begin{array}{c}1.95 \\
(1.39,2.74)^{* *} \\
(\mathrm{n}=76333)^{\mathrm{b}}\end{array}$ & $\begin{array}{c}2.27 \\
(1.44,3.58)^{* *} \\
(\mathrm{n}=7770)^{\mathrm{b}}\end{array}$ & $\begin{array}{c}4.29 \\
(2.57,7.18)^{* *} \\
(\mathrm{n}=7365)^{\mathrm{b}}\end{array}$ & $\begin{array}{c}2.47 \\
(1.74,3.52)^{* *} \\
(\mathrm{n}=7158)^{\mathrm{b}}\end{array}$ \\
\hline
\end{tabular}

$\mathrm{p}<0.05^{*}, \mathrm{p}<0.001^{* *}$

${ }^{a}$ All analyses control for race/ethnicity, sex, the highest level of parental education, geographic region, metropolitan statistical area, baseline cohort year, and baseline measures of past-year alcohol use, past-year marijuana use, and past-year other drug use.

${ }^{\mathrm{b}}$ Sample sizes vary due to missing data on the outcome measures (i.e., AUD, CUD, ODUD, and any SUD symptoms at age 35 )

\section{Opioid use disorder (OUD)}

OUD is defined in the DSM-5 as a problematic pattern of opioid use which leads to significant clinical impairment or distress. ${ }^{150}$ At least two of 11 criteria must be 
observed within a 12-month period, before a diagnosis of OUD is conferred; these criteria are listed below. ${ }^{150}$

1. Opioids are often taken in larger amounts or over a longer period than was intended.

2. There is a persistent desire or unsuccessful efforts to cut down or control opioid use.

3. A great deal of time is spent in activities necessary to obtain the opioid, use the opioid, or recover from its effects.

4. Craving, or a strong desire or urge to use opioids.

5. Recurrent opioid use resulting in a failure to fulfill major role obligations at work, school, or home.

6. Continued opioid use despite having persistent or recurrent social or interpersonal problems caused or exacerbated by the effects of opioids.

7. Important social, occupational, or recreational activities are given up or reduced because of opioid use.

8. Recurrent opioid use in situations in which it is physically hazardous.

9. Continued opioid use despite knowledge of having a persistent or recurrent physical or psychological problem that is likely to have been caused or exacerbated by the substance.

10. Exhibits tolerance.

11. Exhibits withdrawal.

It should be noted that prior to the release of the DSM-V manual, guidelines from the DSM-IV-Text Revision categorized substance abuse and dependence separately. ${ }^{151}$

However, from the time of the publication of the DSM-IV in 1994, several concerns were raised which was related to the classification of substance use disorders, including whether substance use dependence and abuse should be retained as two main disorders. ${ }^{152}$ Ultimately, the decision was made to combine abuse and dependence criteria into one 
disorder in the DSM-V. ${ }^{152}$ (This revised definition was reflected in the NSDUH surveys starting in 2015). ${ }^{153}$

Overdose deaths due to the misuse of prescription opioids and use of heroin occur because of the significant clinical impairment and distress as a result of abuse or dependence on opioids and other substances. ${ }^{154}$ In a meta-analysis by Degenhardt et al., pooled mortality rates (MR) were calculated using data from 51 cohorts all over the world. ${ }^{155}$ All eligible studies had to include measures on the specific causes of death among people who regularly use or are dependent on heroin and other opioids. ${ }^{155}$ Of the 51 studies, 38 provided data on opioid-related overdose mortality, and the investigators determined that the pooled crude MR was 0.65 per 100 person-years (95\% CI: 0.55 , $0.75) .{ }^{155}$

Further complicating this narrative is the fact that those who are dependent, or abuse opioids may also be at higher risk of other substance use disorders (SUD). Wu et al. used nationally representative data from the NSDUH from 2005 to 2013, to compute estimates for the overall prevalence of opioid abuse and dependence among those with OUD $(n=6,125)$, which were $24.73 \%$ and $75.27 \%$, respectively. ${ }^{156}$ The prevalence included all eligible participants (including adolescents), with a total sample size of 503,101 participants. ${ }^{156}$ Additional results from this study revealed that those with a heroin use disorder (HUD) were more likely to have other drug use disorders in the past year than those with a prescription opioid use disorder (Rx-OUD) [aOR (95\% CI): 1.80 $(1.28-2.53)] .{ }^{156}$ 


\section{Mental illness}

Having a mental illness (including depression, anxiety disorders, and mood

disorders) has been shown as a major comorbidity when it comes to opioid misuse. ${ }^{92} \mathrm{~A}$ study on young adult ecstasy polydrug users, aged 18 to 29, was conducted by Keyes et al. using Wave I data from the NESARC. ${ }^{92}$ The purpose of their study was to determine the polydrug use profile in this population and to compute the prevalence of lifetime and past-year DSM-IV Axis I disorders (e.g., depression, mania; panic disorders, social phobia) and Axis II disorders (e.g., personality disorder, antisocial disorder). ${ }^{92}$ The weighted prevalence of having any mood disorder among those who used illicit drugs other than ecstasy, including opioids, was $21.6 \%$, and those who used any other illicit drug were more likely to have a mood disorder compared to individuals who had never used drugs [aOR $(99 \% \mathrm{CI}): 2.4(1.9-3.0)] .{ }^{92}$ Furthermore, the risk of having all other Axis I and Axis II disorders were increased in the illicit drug use population compared to non-drug users. ${ }^{92}$

Extended work regarding psychopathology and opioid use was also conducted using NESARC data from Waves I and II. ${ }^{157}$ The investigators were able to show that among those with no history of psychopathologies, the risk for certain mental disorders significantly increased when past year NMUPO was reported, including substance use disorder [aOR (95\% CI): 3.13 (1.42-6.89)], depressive disorders [aOR (95\% CI): 1.21 (1.11-1.32)], bipolar disorders [aOR (95\% CI): 2.81 (2.47-3.21)], and anxiety disorders [aOR $(95 \% \mathrm{CI}): 1.38(1.03-1.83)]{ }^{157}$

In a recent study by Olfson et al., patient characteristics prior to their opioidrelated death among nonelderly (i.e., less than 64 years of age) Medicaid patients were 
analyzed. ${ }^{158}$ The investigators found that the majority of the decedents had received a diagnosis of chronic noncancer pain conditions in the last year of life $(n=8,050$; $61.5 \%) .{ }^{158}$ Furthermore, a diagnosis of depression $(n=2,383 ; 29.6 \%)$ or anxiety $(n=$ 2,$077 ; 25.8 \%$ ) occurred much more often among the chronic pain patients compared to those without chronic pain. ${ }^{158}$

\section{Employment status}

Current research regarding the role of employment status and opioid-related polydrug use is mixed. There is some evidence to suggest that being employed is associated with the decreased misuse of any prescription drug. ${ }^{159,160}$ However, other studies focus on types of employment where individuals are at higher risk of misusing prescription opioids due to workplace injuries which necessitate long term opioid analgesic treatment. ${ }^{161}$ Conversely, it has also been suggested that workplace injuries may be the result of substances such as prescription opioids and benzodiazepines. ${ }^{162}$ Another important factor that may confound the association between opioid-related polydrug use and employment is the effect of work provided health insurance. While it would be difficult to establish this connection using data from the NSDUH, it has been well established that individuals with health insurance plans generally have a greater level of access to medical care. ${ }^{163-165}$

\section{Health Insurance}

The ability to receive healthcare under the protection of health insurance is a factor that plays into all types of illness, including accidental overdose. Therefore, it 
comes as no surprise that many of the studies revolving opioid-related overdose consider an individual's health insurance type. Hasegawa et al. conducted a retrospective cohort study which sought to quantify the rate of emergency departments (ED) for opioid overdose, and to examine whether frequent ED visits are associated with more hospitalizations, near-fatal events, and in-hospital mortality. ${ }^{166}$ In their multivariable model predicting frequent ED visits for opioid overdose between 2010 and 2011 ( $\mathrm{n}=$ 19,831), those with private insurance, Medicare [OR (95\% CI): 2.06 (1.70-2.51)] and Medicaid [OR (95\% CI): 1.77 (1.47-2.14)] patients were more likely to be admitted into the ED for opioid overdose. ${ }^{166}$

\section{Setting Factors: Environmental Characteristics Associated with Opioid Misuse Urban and rural differences}

Living in rural areas has also been attributed as a risk factor for OPR overdose

morbidity and mortality. ${ }^{167,168}$ Paulozzi et al. analyzed data from the NVSS to quantify changes in drug-poisoning rates in urban and rural communities, and to determine the types of drugs involved in these deaths. ${ }^{167}$ They found that a dramatic increase in opioidrelated mortality from 1999 to 2004 was occurring in areas that were smaller than large central metro areas (i.e., rural areas). ${ }^{167}$ In an observational study by Young et al., rural populations in Kentucky were also determined to have significantly earlier ages of onset for prescription opioids (e.g., oxycodone and hydrocodone), compared to their urban counterparts. ${ }^{168}$

In a more recent study by Rigg and Monnat, data from the 2011 and 2012 NSDUH was analyzed to determine whether differences existed in prescription opioid 
misuse among living, nationally representative adults. ${ }^{161}$ A difference of proportion t-test analysis comparing the two populations found that the urban communities had a significantly $(p=0.0002)$ higher proportion of NMUPO $(5.7 \%)$ compared to rural communities (4.9\%). ${ }^{161}$ Additionally, the unadjusted and adjusted logistic regression models reported by the investigators showed a significant decrease in the odds of rurality in predicting NMUPO. ${ }^{161}$

However, it should be noted that there are regional differences in opioid misuse and overdose rates. ${ }^{89}$ In a briefing released by the Assistant Secretary for Planning and Evaluation (ASPE), areas where the prevalence of drug overdose deaths and opioid prescriptions include both rural and urban areas, more specifically Appalachia, New England, and certain areas of the West and Midwest. ${ }^{89}$ And although it was shown that poverty and unemployment was greatly associated with drug overdose deaths and opioidrelated hospitalizations, these relationships remained clustered in specific areas of the U.S. ${ }^{89}$ However, this association was not constant because there were still some counties that did not have high substance use and opioid prevalence indicators despite having high poverty and unemployment (see Figure 2.7). ${ }^{89}$

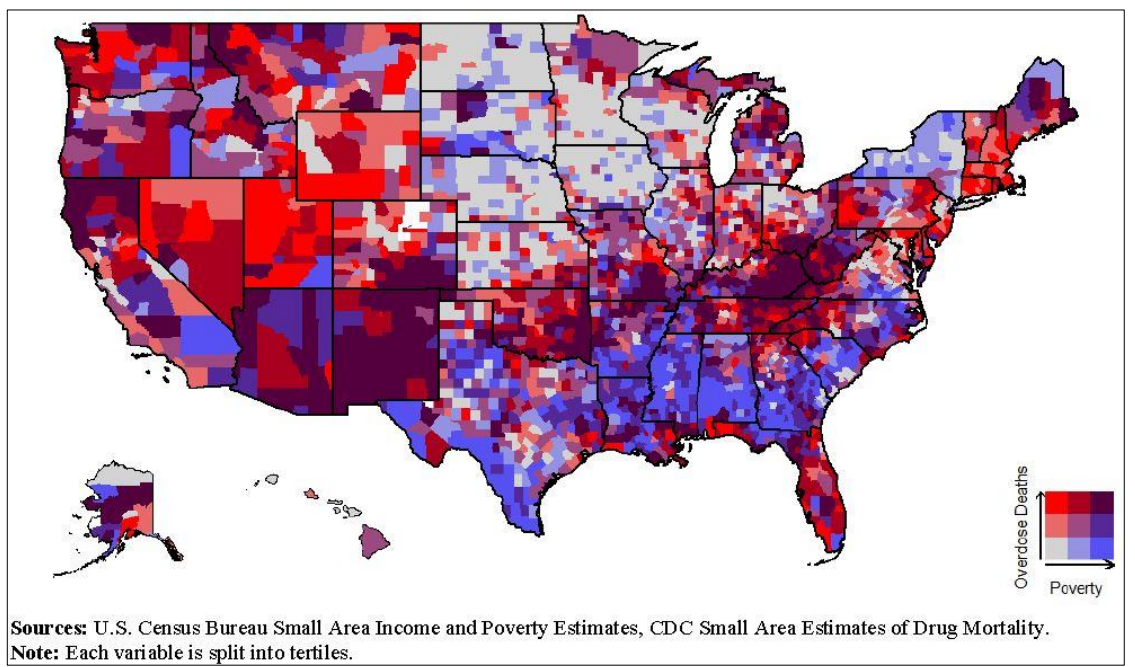

Figure 2.7 | Drug Overdose Death Rates and Poverty Rates, United States $2016^{89}$ 


\section{Residential Transience}

Residential transience is a concept related to homelessness and housing instability. ${ }^{169}$ It has been shown that individuals who are either homeless or have unstable housing options are more likely to report the use of substances, including heroin and prescription opioids. ${ }^{169-173}$ Possible mechanisms for this relationship are complex, and may include life stress and social disruption (e.g., loss of employment, starting a new routine, financial distress) or may even have occurred as a result of substance use. ${ }^{174,175}$ To date, very limited studies examine the association between polydrug use among PWUO and residential transiency, especially studies using a nationally representative sample. However, the link between housing instability and mortality has been previously established. In a study by Baggett et al., adults aged 18 years and older were retrospectively sampled between January 2003 to December 2008 from the Boston Health Care for the Homeless Program (BHCHP) to assess the overall and cause-specific mortality rates. ${ }^{173}$ The investigators found that drug overdose accounted for approximately $16.8 \%$ of deaths among adults who were less than 45 years of age [crude mortality rate $(95 \% \mathrm{CI}): 242.1(210.1-274.2)] .{ }^{173}$

\section{What This Study Contributes}

Currently, limited studies have documented the trends in substance use among different subgroups of PWUO. Studies found in this arena have assessed polydrug use on those who have reported the nonmedical use of prescription opioids, have reported primary heroin use, or have focused on the co-use of one or two other substances (e.g., alcohol, benzodiazepines, marijuana). ${ }^{19,176}$ Additionally, the trend in polydrug latent 
classes from 2002 to 2017 will further elucidate the trajectories of opioid-related subpopulations. Finally, associations between covariates belonging to the drug, set, and setting framework and polydrug latent classes will help to characterize the polydrug latent classes among PWUO who may be higher at risk for overdose. 


\section{CHAPTER III}

\section{RESEARCH DESIGN AND METHODOLOGY}

\section{Introduction}

This chapter will provide a detailed description of the NSDUH, including the eligibility criteria of the participants and the complex sampling procedure used by the collection agency, the Center for Behavioral Health Statistics and Quality (CBHSQ). Additionally, the dependent and independent variables that will be used in this study will be defined following a discussion on the conceptual framework used to guide this study. Data analysis related to the construction of the dependent variables will be provided under the analytical strategy of their respective Aim.

\section{National Survey of Drug Use and Health (2002-2017) ${ }^{40}$ Description and source of the study population}

The NSDUH is a nationwide study that is sponsored by SAMHSA. The survey is conducted every year by the Research Triangle Institute (RTI International) and provides information on tobacco, alcohol, and other types of substance use, along with mental health and other health-related issues in the United States. Data collected in the survey is authorized by Section 505 of the Public Health Service Act, which states that the national 
incidence and prevalence of the various forms of mental illness and substance abuse be collected every year ${ }^{6}$. As a cross-sectional study, participants are not followed up every year, which prohibits any analyses related to establishing temporality or causality.

Since 1999, data collection consisted of a computer-assisted self-interview (CAI) by a field interviewer and an audio computer-assisted self-interview (ACASI). Questions included age at first use, lifetime, annual, and past month usage for major substance classes such as marijuana, cocaine, heroin, hallucinogens, inhalants, tobacco, alcohol, and a variety of prescription medications (e.g., pain relievers, tranquilizers, stimulants, and sedatives). Additionally, participants were questioned about their substance abuse treatment history, health care access and coverage, and standard demographic variables (e.g., gender, race, ethnicity, etc.). And because the dataset used in this study is publicly available, all files have been deidentified (including geographic variables) for the protection of all respondents.

\section{NSDUH eligibility criteria}

The study population consists of noninstitutionalized U.S. civilians who are at least 12 years of age. However, about three percent of the U.S. population are excluded from this survey, including members of active-duty military and individuals living in institutional group quarters (e.g., hospitals, prisons, nursing homes, and treatment centers). After the final dwelling units (DU) are selected, field interviewers are dispatched to homes in a random fashion. Potential participants are asked a series of screening questions and recorded on the computer. Screening questions covered age,

642 U.S.C. $\S 505(2012)$ 
race, gender, DU residency status, and their spoken language. ${ }^{177}$ Because the survey is only available in English and Spanish, households where neither were spoken were excluded from participation.

\section{Sampling procedure}

Since 1999, independent, multistage area probability sampling has been used for all 50 States and the District of Columbia. Although slightly different target populations were recruited from year to year, each year averaged over 65,000 participants. ${ }^{178}$ The general concept of the multistage sampling involves the division of a State into progressively smaller geographic areas until individuals within dwelling units are screened and formally recruited (as shown in Figure 3.1). It must be noted that slight variations of the sampling procedure do exist across different time frames and are summarized in Table 3.1 at the end of this section.

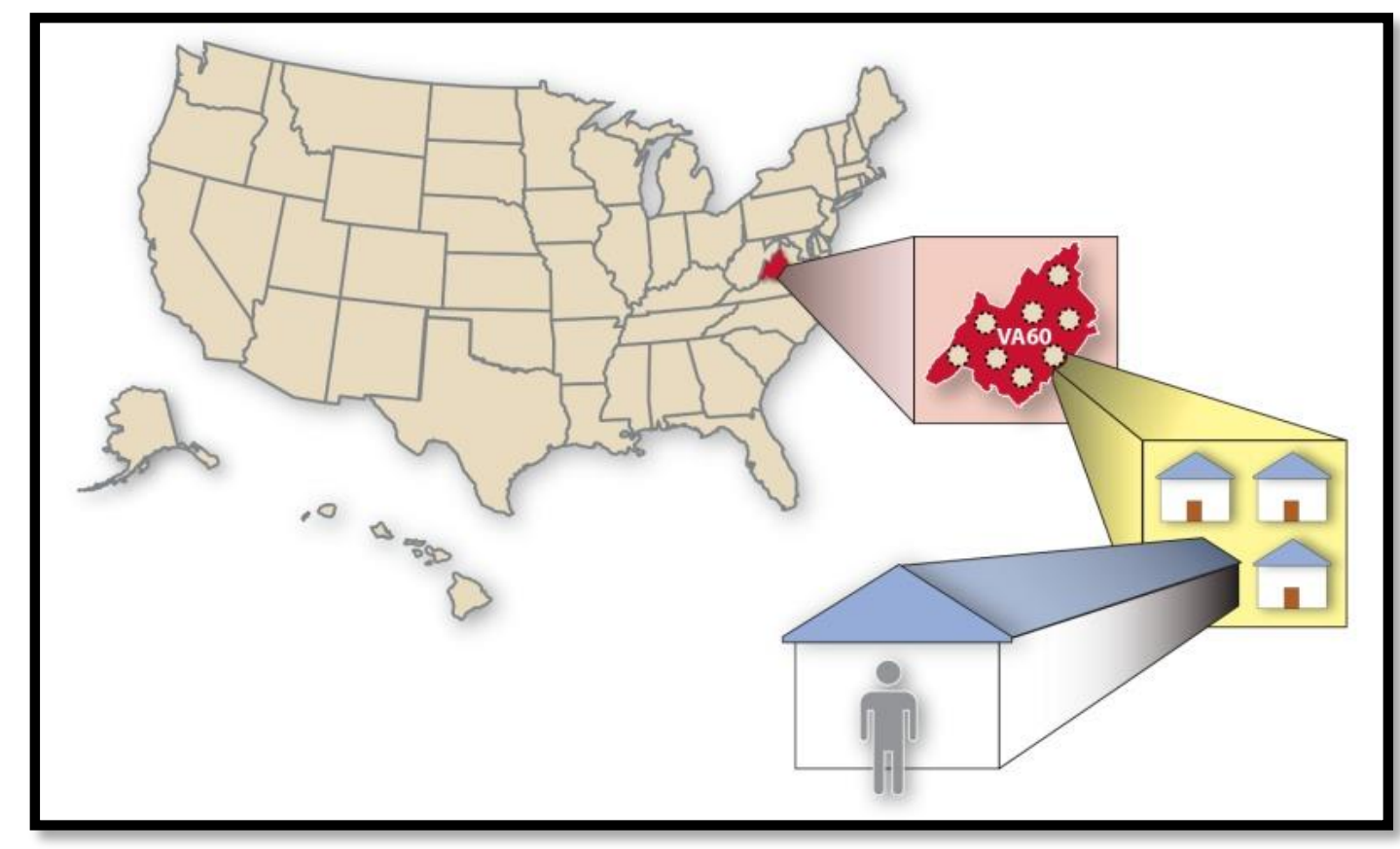

Figure 3.1.| Multistage Area Probability Sampling Procedure for the NSDUH 
Table 3.1 Summary and Differences in Sampling Procedures in the NSDUH, 2002 to 2017

\begin{tabular}{|c|c|c|c|}
\hline Stage & 2002 - 2004 & 2005 - 2013 & 2014 - 2017 \\
\hline 1 & $\begin{array}{c}\text { Large sample States (CA, FL, } \\
\text { NY, TX, IL, MI, OH, PA) } \\
\text { divided into 48 field } \\
\text { interviewer (FI) regions, while } \\
\text { all other States divided into 12 } \\
\text { FI regions. }\end{array}$ & $\begin{array}{c}\text { Approximately equally sized state sampling regions (SSRs) formed } \\
\text { based on composite size measures. Once SSRs were determined, 48 } \\
\text { census tracts within the SSR were chosen. }\end{array}$ \\
\hline 2 & $\begin{array}{c}\text { Partitioning of each FI region } \\
\text { into groups of adjacent census } \\
\text { blocks called segments. }\end{array}$ & $\begin{array}{c}\text { Selected census block } \\
\text { groups were partitioned into } \\
\text { segments, which are defined } \\
\text { by joining contiguous census } \\
\text { blocks within each census } \\
\text { block group. }\end{array}$ & $\begin{array}{c}\text { Adjacent census block groups were } \\
\text { aggregated within selected census } \\
\text { tracts. A total of 48 census block } \\
\text { groups were chosen within each } \\
\text { SSR. }\end{array}$ \\
\hline 3 & Dwelling units (DUs) were selected within each segment. & $\begin{array}{c}\text { pelected census block groups were } \\
\text { partitioned into smaller areas called } \\
\text { segments, which are defined by } \\
\text { joining contiguous census blocks } \\
\text { within each census block group. }\end{array}$ \\
\hline 4 & Individuals within screened DUs are chosen based on age \\
group. & $\begin{array}{c}\text { Dwelling units (DUs) were selected } \\
\text { within segments. }\end{array}$ \\
\hline 5 & n/a & $\begin{array}{c}\text { Individuals within screened DUs } \\
\text { are chosen based on age group. }\end{array}$ \\
\hline
\end{tabular}




\section{Format of self-administered substance use sections}

The 2002 to 2014 NSDUH survey had a total of 12 substance use sections, which may be broadly characterized as follows: 2 ) legal substances with age restrictions (i.e., tobacco, alcohol); 2) illicit substances (i.e., marijuana, cocaine, crack, heroin, hallucinogens, inhalants); and 3) prescription drugs (i.e., pain relievers, tranquilizers, stimulants, sedatives). A $13^{\text {th }}$ substance use section was added starting in 2015 , for illicit methamphetamines, which was not previously captured under the broader stimulant substance category. Another motivation to include this as a separate category was to maximize the likelihood of the participant's self-report of illicit stimulants (e.g., crystal methamphetamine) since the context of the original 'stimulants' module revolved around prescription stimulants. ${ }^{153}$ Questions were designed to ask the participant about his or her frequency of use related to their lifetime use, past year use, and past month use. Many of the substance use modules are general when asking about the participant's history of use; for example, in the tobacco section, the question that appears on the self-administered questionnaire include: "have you ever smoked part or all of a cigarette?", to which the respondent may select either 'yes' or 'no.'

However, it should be noted that many of the variables used in this study were imputed based on responses from a different question then recoded to simplify analysis efforts. An example of this is for past month cigarette use (стямоN); the original survey question asks the participant "how long has it been since you last smoked part or all of a cigarette?". ${ }^{40}$ For all other core substance modules, questions are asked in almost the same sequence, with extremely similar (if not exact) wording as the example provided above. The only modules that deviated from the format of the tobacco, alcohol, 
marijuana, and cocaine modules were those that included a greater variety of substances namely, hallucinogens and inhalants. However, each substance (i.e., LSD, PCP; amyl nitrite, lighter fluid) included within these two modules were given their own 'ever used' question, with a collective "how long has it been since you last used [hallucinogens/inhalants]...?”.

\section{Survey design changes}

Changes in the NSDUH have occurred throughout the life of its administration, and several must be noted to provide a methodological context of the possible changes in the frequencies in reported substance use behavior from 2002 to 2017. Although this study does not include data prior to 2002, improvements to the 2002 NSDUH were made based on data collection changes that were instituted for the 1999 survey (then called the National Household Survey on Drug Abuse; NHSDA)..$^{40}$ Prior to the 1999 NHSDA, participants were asked to complete the survey using a paper-and-pencil interviewing (PAPI) method; additionally, the sampling strategy went from a strictly national design to being state-based (as detailed in the previous Sampling procedure section). ${ }^{40}$ For the 2002 survey, a name change was instituted, and the NHSDA became henceforth known as the National Survey on Drug Use and Health. ${ }^{40}$ Other changes in 2002 included the use of a $\$ 30$ incentive, the improved data collection and quality control procedures (that had been introduced in the 2001 survey), and the use of the 2000 decennial census for sample weighting procedures. ${ }^{40}$

In 2015, the questionnaire underwent several substantial changes to improve the quality of the NSDUH data and to address the changing needs of policymakers and 
researchers about substance use and mental health issues. ${ }^{179}$ Of notable concern for this study, questionnaire changes in 2015 included the following ${ }^{179}$ :

- The response categories were revised in the question about the highest level of education that was completed, including whether respondents received a high school diploma, General Educational Development (GED®) certificate of high school completion or college degree.

- Smokeless tobacco sections (snuff and chewing tobacco) were combined into one section. "Snus" was added as an example of smokeless tobacco.

- The threshold for binge alcohol use was changed from five or more drinks to four or more drinks for female respondents.

- Questions that previously were included in the special drugs module for hallucinogens were moved to the core hallucinogens module. This includes ketamine; tryptamines (dimethyltryptamine [DMT], alpha-methyltryptamine [AMT], and 5-MeO-DIPT [N, N-diisopropyl-5-methoxytryptamine], also known as "Foxy"); and Salvia divinorum.

- A new methamphetamine module was added, separate from questions about misuse of prescription stimulants.

- The approach and definition for measuring the misuse of prescription drugs were revised to include questions about any use of prescription drugs in addition to questions about misuse (i.e., nonmedical use), and, for questions about misuse, to focus on specific behaviors that indicate misuse.

- Prescription drugs that previously were included elsewhere in the main questionnaire (i.e., Adderall®, Ambien ${ }^{\circledR}$ ) were moved to the appropriate prescription drug module.

- Demographic questions about moves in the past 12 months, immigrant status, marital status, education, and employment were moved from being intervieweradministered through CAPI to being self-administered through ACASI.

However, it should be noted that changes beyond those mentioned in this section have been seen to occur, particularly in the operational definition of certain mental health disorders. Changes outside of those detailed in this section are addressed in each of the following sections that discuss the variables used in this study. 


\section{The Current Study: Polydrug Use in PWUO, 2002-2017}

\section{Inclusion criteria}

Because the focus of this study is on the reported polydrug use behaviors in those who use opioids, there were two delimitations imposed on the entire NSDUH study population. First, only individuals that reported being 18 years of age or older at the time of the survey were included. Adolescents aged 12 to 17 were excluded because of two reasons: 1) modules related to mental health and substance use behavior were not comparable between the adult and adolescents and 2) adolescents are less likely to report having used either heroin or prescription pain relievers, despite reports that the misuse of opioids is the fastest growing drug problem in this age group. ${ }^{180,181}$ Second, only those who answered 'yes' to having used heroin or misused prescription pain relievers in the past 30 days were included in the study. Past month measures were used to limit recall bias and to restrict the time frame to include only those currently using the substances. Once these restrictions were imposed, the total number of participants surveyed from 2002 to $2017(\mathrm{~N}=621,571)$ decreased to $16,985(2.73 \%)$.

\section{Definition of the drug, set and setting variables}

All variables assessed in this study are taken from the publicly available NSDUH datasets from 2002 to 2017. Again, all data are self-reported measures by the participant. However, picture cards were used during portions of the survey that asked about prescription drugs (e.g., pain relievers, tranquilizers, sedatives) to help participant recall. The following subsections will describe all variables that will be used based on their designation within the drug, set, and setting conceptual framework (discussed in Chapter II). 


\section{Drug variables}

All the core substances surveyed in the NSDUH will be used in the analysis (i.e., tobacco, alcohol, marijuana, cocaine, crack, heroin, hallucinogens, inhalants, pain relievers, tranquilizers, stimulants, sedatives). It should be noted that the methamphetamine module was excluded from analyses because it was only included in the survey beginning in 2015 .

\section{a. Alcohol use}

The original alcohol variable used in this study reflected past month use with four levels: 1) heavy alcohol use, 2) "binge" but not heavy use, 3) past month but not "binge," and 4) did not use alcohol in the past month. This categorical variable was recoded by the CBHSQ by combining three separate variables: 'past month binge alcohol use,' past month heavy alcohol use,' and 'past month alcohol use.' (The heavy alcohol use category also included binge alcohol users; however, binge alcohol users excluded heavy alcohol users, and past month alcohol users were individuals who were not/never "binge" alcohol users). The data for these three separate variables were originally asked in the interview using the following question: "on the ... days that you drank during the past 30 days, how many drinks did you usually have? Count as a drink a can or bottle of beer; a wine cooler or a glass of wine, champagne, or sherry; a shot of liquor or a mixed drink or cocktail."

The responses to this question were entered as a continuous variable but later recoded by CBHSQ with specific definitions for 'binge drinking' and 'heavy drinking.' 'Binge drinking' is when an individual has had five or more drinks on the same occasion 
on at least one day in the past 30 days, while 'heavy drinking' is defined as having had five or more drinks on the same occasion on each of five or more days in the past month. But starting in 2015, female participants were asked to report the number of days that they had four or more drinks on the same occasion as the definition of 'binge drinking.' 153

After the initial use of the four-category alcohol use variable in the prevalence analyses, it was observed that heavy and binge alcohol had been reported at higher proportions, and the decision was made to create two dummy variables from the original four-category variable. Heavy alcohol use and binge alcohol use were recoded into dichotomous (yes/no) variables for past 30-day use. It should be noted that the dichotomous (yes/no) variable for past 30-day heavy drinking was the only variable included in the LCA model for two reasons:

1. Heavy alcohol use was significant in the bivariable association among most opioid use groups (i.e., PPR-only and heroin-only). (See Supplemental Table 4.13 in Appendix A).

2. It included binge alcohol users, which has been found significantly associated with PWUO.

\section{b. Cigarettes and other tobacco products use}

Questions related to the use of cigarettes and other tobacco products were contained in the same overall substance module titled "Tobacco." For cigarette use, respondents were asked, "Now think about the past 30 days, that is, from [DATEFILL] up to and including today. During the past 30 days, have you smoked part or all of a cigarette? How long has it been since you last smoked part or all of a cigarette?’. 
Responses were later recoded by the CBHSQ into binary (yes/no) variables that indicated past year, past month, and lifetime use.

While the final variable used in this study for other tobacco product use was a dichotomous (yes/no) variable, several variables were used to create it. For the 2002 to 2014 surveys, other tobacco products consisted of cigars, pipe tobacco, smokeless tobacco (including separate questions for chewing tobacco or snuff), and the original interviews for each of these were queried the same way as the cigarettes. For surveys that occurred after 2014, the changes in survey methodology led to the removal of the individual chewing tobacco and snuff questions, leaving only broader questions to assess smokeless tobacco use, such as: "The next questions are about your use of "smokeless" tobacco such as snuff, dip, chewing tobacco, or 'snus.' Have you ever used "smokeless" tobacco, even once?' or 'Now think about the past 30 days -- that is, from [DATEFILL] up to and including today. During the past 30 days, have you used "smokeless" tobacco, even once? How long has it been since you last used "smokeless" tobacco?" The responses for cigars, pipe tobacco, and smokeless tobacco were then recoded by CBHSQ to reflect dichotomous (yes/no) variables.

\section{c. Hallucinogen and inhalant use}

For hallucinogens and inhalants, interviewers were trained to ask the participants: whether they had taken specific examples for different hallucinogens or inhalants (in their respective module); those who responded in the affirmative were then asked when the last time the participant had used different types of tranquilizers, or simply, an inhalant. These responses were then imputed into the three frequency levels (e.g. 'ever used,' 'past 
year,' and 'past month'), and finally recoded into dichotomous (yes/no) variables. For this study, one new variable was created using separate NSDUH variables for past month use of hallucinogens and inhalants. This was done for two reasons: 1) to increase overall sample size for this category and 2) combining the two substances was supported by evidence from a study that evaluated their psychometric properties using factor analysis and item response theory (IRT) analysis. ${ }^{182}$ The results of this study suggested that it would be appropriate to combine hallucinogen and inhalant/solvent abuse and dependence criteria in future editions of the Diagnostic and Statistical Manual of Mental Disorders (DSM). ${ }^{182}$

\section{d. Tranquilizer and sedative use}

Data for tranquilizer and sedative use were collected in similar fashions before and after 2015, with the largest difference being the use of showcards with pill images in surveys before 2015. However, regardless of the survey year, participants were asked: "have you ever, even once, used any type of prescription tranquilizer/sedative that was not prescribed for you or that you took only for the experience or feeling it caused?". Those who responded 'yes' were followed up with: "how long has it been since you last used any prescription tranquilizer that was not prescribed for you or that you took only for the experience or feeling it caused?". Depending on the response (i.e., 'within the past 30 days', 'more than 30 days but within the past 12 months', or 'more than 12 months ago'), dichotomous (yes/no) variables for 'ever use', 'past year use', and 'past month use' were recoded by CBHSQ. For this study, another level of recoding was completed to combine both of these substances into one past-month use variable because 
benzodiazepines can be classified into both groups, and have been found to play a major role in opioid overdose rates. ${ }^{183}$

\section{e. Use of all other core substances}

The remaining core substance modules followed the same interview structure as those described above. For this study, the final dichotomous (yes/no) variables for past month use of marijuana, cocaine, crack, and stimulants were kept as their original definitions and not recoded for analyses.

\section{Set variables}

All variables within this designation were used as independent variables to examine their associations with polydrug latent class membership. Demographic variables assessed in this study were age, sex, race/ethnicity, total household income, education status, and marital status.

\section{a. Age at time of survey administration}

'Age at the time of the survey' was calculated from the stated birthdate of the respondent; the interviewee was asked "what is your date of birth?" and the computerassisted interview program automatically calculated the age by "subtracting" the participant's date of birth from the date of the interview. The interviewer then confirmed the age with the participant by asking "I have entered your date of birth as [age]. Is this correct?" This information was then recoded by the data management team into several categorical age variables, but the age variable being used in this study originally included 
five levels. However, after the inclusion criteria were imposed for this study, the number of age groups decreased to just four levels: 18 to 25 years, 26 to 34 years, 35 to 49 years, and 50 and older. But due to small sample sizes in the older age categories, age was further recoded. Therefore, the final number of levels in the age variable was three: 1) 18 to 25 years, 2) 26 to 34 years, and 3) 35 years and older.

\section{b. Sex}

Based on the final computer-assisted interview (CAI) specifications handbook, interviewers were instructed to record the respondent's gender, with only two options: male or female. ${ }^{177}$ (It is unclear whether a formal question was asked of the respondent). For this study, sex was left as a dichotomous variable with these two levels.

\section{c. Race/ethnicity}

To establish a participant's race/ethnicity, they were first asked: "are you of Hispanic, Latino, or Spanish origin or descent?"; if they answered 'yes,' a showcard with a list of specific groups (e.g., Mexican, Puerto Rican, Dominican, etc.) was shown, and the participant was asked to select the number that corresponded to the appropriate Hispanic, Latino, or Spanish population. If they answered 'no' to that question, they were shown a different card with non-Hispanic/Latino groups (e.g., White, Black, Asian, etc.) and asked to select the appropriate number that corresponded to the nonHispanic/Latino group. Although the original race/ethnicity variable included seven groups, small sample sizes in the 'Non-Hispanic Native American or Alaskan Native', 'Non-Hispanic Hawaiian or other Pacific Islander', 'Non-Hispanic Asian', and 'Non- 
Hispanic more than one race' populations necessitated the recoding of these groups into a general 'Other race/ethnicity' category. The final number of race/ethnicity categories was four: 1) Non-Hispanic White, 2) Non-Hispanic Blacks, 3) Hispanics, and 4) other race/ethnicities.

\section{d. Total household income}

Respondents were originally asked, "before taxes and other deductions, was your total personal (or total personal income from all sources) during [current year] more or less than $\$ 20,000$ ?'. For those who answered 'yes,' the following question was asked: “Of these income groups, which category best represents total personal during [current year]?”. Yearly household income was originally a categorical variable with seven levels: less than $\$ 10,000 ; \$ 10,000$ to $\$ 19,999 ; \$ 20,000$ to $\$ 29,999 ; \$ 30,000$ to $\$ 39,999$; $\$ 40,000$ to $\$ 49,999 ; \$ 50,000$ to $\$ 74,999$; and $\$ 75,000$ or more. However, this variable was recoded to reflect a total of three levels due to small sample sizes. The final three levels were: 1) less than $\$ 30,000,2) \$ 30,000$ to $\$ 74,999$, and 3) $\$ 75,000$ or more. The recoding of these levels were guided by income levels used in previous research related to substance use. ${ }^{19,184}$

\section{e. Employment status}

Because employment status was measured differently in surveys that occurred prior to 2015, two variables were used to indicate employment status in this study. Pre2015 surveys determined employment status by asking respondents whether they worked in the past week, whether they usually work 35 or more hours per week, and (where 
relevant), reasons why they did not work in the past week. The responses to these questions were then recoded into a 9-level categorical variable and finally recoded into a 4-level variable, which is described at the end of this section. In surveys conducted starting in 2015, respondents were asked a variety of questions to establish their employment status. These questions were:

1. Do you usually work 35 hours or more per week at all jobs or businesses?

2. The next questions are about working. Did you work at a job or business at any time last week?

3. Did you work at a job or business at any time during the past 12 months?

4. During the past 30 days, did you make specific efforts to find work? Include any contacts you made with anyone about a job, sending out resumes or applications, placing or answering ads. Do not include only reading job ads.

5. Which one of these reasons best describes why you did not have a job or business last week?

6. Which one of these reasons best describes why you did not work last week?

The answers to these six questions were then imputed by the CBHSQ and recoded into the four-category variable that was used in this study: 1) employed full time, 2) employed part-time, 3) unemployed, and 4) other (including not in the labor force). For this study, no additional recoding was performed on the four-category variable.

\section{f. Education status}

The original survey question asked the participant "what is the highest grade or year of school you have completed?". Twenty answers were possible and ranged from 'no school completed' to 'doctorate degree' and 'professional degree.' This was then imputed into nine levels and finally recoded into four levels: less than high school, high school graduate, some college or associate degree, and college graduate. Again, guided 
by previous research, education was further collapsed into three categories: 1) less than high school, 2) high school graduate, and 3) greater than or equal to college. ${ }^{19,184}$

\section{g. Marital status}

At the interview, respondents were asked: “Are you now married, widowed, divorced or separated, or have you never married?", then imputed into four levels: married, widowed, divorced or separated, and never been married. For this study, marital status was recoded into two levels: married and not married. This recode was used because of the social implications of being married versus not married as it related to substance use, which has been explored in previous research. ${ }^{185-187}$

\section{h. Age at prescription pain reliever initiation}

Two variables were used to recode for 'age at prescription pain reliever initiation' due to the differing survey methodologies after the 2014 NSDUH survey. From 2002 to 2014, participants were simply asked: "how old were you the first time you used any prescription pain reliever that was not prescribed for you or that you took only for the experience of feeling it caused?" After 2014, participants were asked, “at any time before [the past 12 months], did you ever use any prescription pain reliever in a way a doctor did not direct you to use it?'. For those who responded 'no,' they were labeled as past year initiates, and their age during the past 12 months at the time of use was entered. These two variables were originally entered as continuous values but were recoded for this study into three-levels: 1) less than 18 years of age, 2) 18 to 25 years old, and 3) 26 years of age and older, to represent adolescence, early adulthood, and adulthood. 


\section{i. Opioid use disorder}

The past year opioid use disorder (OUD) variable was a single recoded variable in NSDUH starting in 2016. The CBHSQ used several different responses from questions within the substance dependence and abuse module. Several different questions were asked in the module to classify whether an individual had either a heroin use disorder (i.e., dependence or abuse), or pain reliever use disorder related to their misuse of prescription pain relievers in the past year, or if they had both disorders. The questions were formulated based on the DSM definition of OUD, and included questions such as: "did you continue to use heroin even though you thought it caused problems with family or friends?" or "during the past 12 months, did you notice that using the same amount of prescription pain relievers had less effect on you than it used to?".

But because 2002 to 2015 NSDUH surveys did not already have the single recoded variable that can be found in the 2016 and newer surveys, additional levels of recoding had to be completed for this study to harmonize the OUD variable. For starters, the variables for past year pain reliever dependence and abuse had to be combined into a dichotomous (yes/no) variable from 2002 to 2015; this was also done with the past year heroin dependence and abuse variables for 2002 to 2015 . These two dichotomous variables were then combined into a four-level categorical variable that the 2016 and newer variable contained: 1) only heroin dependence or abuse, 2) only pain reliever dependence or abuse, 3) heroin and pain reliever dependence or abuse, and 4) neither heroin nor pain reliever dependence or abuse. However, due to the small sample size, this four-level variable was collapsed into a dichotomous (yes/no) variable indicating that the participant had abused or was dependent on either heroin or PPRs. 


\section{j. Depression}

Two variables from the NSDUH were used as the 'depression' variable in this study. The first was drawn from the 2005 to 2007 NSDUH, while the second was drawn from surveys starting in 2008. (Prior to 2005, NSDUH did not ask specific questions about depression). From 2005 to 2007 , depression was included as a part of the 'Pregnancy and Health Care' module and was ascertained by asking: "Which, if any, of these conditions, did a doctor or other medical professional tell you that you have in the past 12 months?". The response for the section was then recoded by the CBHSQ to reflect a yes/no variable for 'past year depression.' Questions related to adult depression was separated into its own module starting in 2008 with more detailed questions to try and ascertain whether a respondent had depressive symptoms. Additionally, 'depression' was renamed 'major depressive episode' which was defined as having five or more of the following nine criteria:

1. Having felt sad, empty, depressed, or discourage most of the day

2. Having lost interest or pleasure in most things

3. Having changes in appetite or weight

4. Having sleep problems

5. Having others notice that the respondent was restless or lethargic

6. Having felt tired or low in energy nearly every day

7. Having felt worthless nearly every day

8. Being unable to concentrate or make decisions

9. Having any thoughts or plans of suicide

This variable was also later recoded into a dichotomous (yes/no) variable by the CBHSQ. The final variable used in this study was also left as a dichotomous (yes/no) variable. 


\section{k. Health insurance status}

The variable used in this study related to the current health insurance status of the participant was a dichotomous (yes/no) variable that was imputed from multiple variables in the dataset. These variables were derived from separate interview questions and related to whether the participant had different health insurance plans (e.g., Medicaid/CHIP, Medicare, private health insurance, etc.). In order to be classified as having no insurance, the participant must have answered 'no' to all questions regarding different health plans.

\section{Setting variables}

\section{a. County type}

Two county type variables were used for the analyses in this study because the definition of the county variable beginning in 2015 (COUTYP4) relied upon the 2013 Rural/Urban Continuum Codes. This replaced the county variable used prior to 2015 (COUtyp2), which had been based on the 2003 Rural/Urban Continuum Codes. The ruralurban continuum codes are an effort by the Economic Research Service at the U.S. Department of Agriculture to measure the degree of rurality or relative isolation, in a county and are based on their metropolitan-nonmetropolitan status and size of their metropolitan or urban populations. ${ }^{188}$ Although not completely comparable, the two variables were renamed as one variable (COUNTY), since they are based on similar concepts, with the same three levels are the original variables: 1) large metro, 2) small metro, and 3) nonmetro. 


\section{b. Residential transience}

Residential transience was determined by a single question in the non-core demographics portion of the NSDUH. Participants were asked "how many times in the past 12 months have you moved? Please include moves from one residence to another within the same city/town as well as those from one city/town to another." The responses were entered as a continuous variable but later recoded as a four-level categorical variable: 1) none, 2) one time, 3) two times, and 4) three or more times. For this study, the last two levels were collapsed as one, leaving a total of three-levels in the analysis.

\section{Data Analysis Methods}

\section{Analyses using complex survey data}

The section will address the sampling weight variables that were used for all analyses in this study. As previously stated, NSDUH utilizes a complex 50-state sampling design using independent, multistage area probability sampling. This required specific statistical coding that can handle the different sampling weights, which is not possible with regular coding in statistical software since it would treat the data as if it were a simple random sample. ${ }^{189}$ In SAS 9.4, coding that incorporates survey weights begin with the prefix 'survey'; for example, a regular SAS procedure to calculate simple means and standard deviations is proc means, while proc surveymeans, is used when sample weights are available. ${ }^{189}$ The advantage of using 'survey-' procedures is that it allows the computation of variance estimates using complex multistage survey design with stratification, clustering, and unequal weighting. Additionally, it provides a choice of variance estimation methods, including Taylor series linearization. Consequences of 
ignoring sample weights in complex survey data are inaccurate point estimates and inaccurate standard errors. ${ }^{189}$ In the public-use NSDUH files, the following variance estimation variables are included:

- variance estimation stratum (VESTR)

- variance estimation cluster replicates (VEREP)

- final analysis weight (ANALWT_C)

Because several years of data are being analyzed in this study, a new analysis weight variable was created by dividing 'ANALWT_C' by the total number of years (i.e., 16 years) included in the study, as specified in the NSDUH codebook. ${ }^{40}$ This results in a final person-level analysis weight which is more appropriate to use across successive years. ${ }^{40}$

\section{Joinpoint analysis}

For Aims 1 and 2, joinpoint regression analysis was used to determine whether a significant trend (or trends) exist in the weighted prevalence of reported past month substance use. Joinpoint regression was performed using the National Cancer Institute's (NCI) Joinpoint Trend Analysis software, version 4.7.0.0 (updated February 2019). Joinpoint regression is a statistical technique that identifies points in time where linear trends change significantly in both magnitude or direction. ${ }^{190}$ Results from this type of regression analyses would allow us to determine the specific year(s) where the trajectory of the weighted prevalence significantly changes. ${ }^{191}$ Although the NCI software selects the final model using a variety of tests which is selected by the user, the permutation test was chosen for this study because it generally produces parsimonious results and has the 
longest track record of all the other methods, such as traditional Bayesian Information Criterion (BIC) and data-driven BIC methods. ${ }^{192}$ It should also be noted that if the prevalence of the test variable was normally distributed, log-transformation was not selected in the software, and the analysis reported the slope (m). However, if the prevalence of the test variable is not normally distributed, the data were log-transformed, which yielded a measure called the 'annual percent change' (APC) instead of reporting slopes.

\section{Latent class analysis}

LCA is a nonparametric approach that assigns class membership on a probabilistic basis depending on the response to a set of discrete indicator variables. ${ }^{193}$ Although LCA has been likened to factor analysis, it is a considered a person-centered

approach because the focus is on the relationships in a specific subpopulation. ${ }^{194,195}$ Another difference between LCA and factor analysis is the nature and distribution of the latent variable; LCA uses categorical variables which have multinomial distributions, while classic factor analysis uses continuous (i.e., dimensional) variables with a normal distribution. ${ }^{194}$ Using a person-centered approach is useful in establishing associations with a multitude of covariates where populations are the unit of measurement. In contrast, variable-centered approaches focus on the relationship between individual variables. ${ }^{195}$ Additionally, LCA is useful in representing heterogeneity in developmental trajectories using longitudinal data. ${ }^{195}$ 


\section{Time variable}

A continuous time variable (in years), survyr, was created within each separate NSDUH dataset from 2002 to 2017. While this variable was meant to remain as a continuous variable during analysis, the low sample size for a few independent variables necessitated the recoding of the variable into periods. The period variable used for this study consisted of four levels: 1) 2002 to 2005, 2) 2006 to 2009, 3) 2010 to 2013, and 4) 2014 to 2017. However, it should be noted that because joinpoint analysis was conducted to examine linear trends of prevalence, the continuous survey year variable (i.e., survyr) was used.

\section{Aim 1 analytic strategy}

Aim 1 sought to examine the prevalence of polydrug use among all PWUO and each opioid use group in the U.S. from 2002 to 2017. The two outcome variables used for Aim 1 were categorical. The first outcome variable was a recoded dichotomous (yes/no) variable that designated PWUO-status (pwuo). The second outcome variable was a recoded three-level categorical variable that designated membership in each opioid use group (aim1). Two subaims and their corresponding analytic strategies are described below to achieve Aim 1. SAS version 9.4 (Cary, NC, USA) was used as the main statistical analysis software for the whole study.

Subaim 1.1: To ascertain the prevalence of the all PWUO as well as opioid use groups (i.e., heroin only, prescription opioid only, and combination opioids) and determine whether they differ over time. 
The overall and period-stratified weighted prevalence (\%) of all PWUO and opioid use groups was calculated using proc surveyfreq. The period-stratified frequencies were then plotted on a line graph to visualize changes over time. A RaoScott Chi-square $\left(\chi^{2}\right)$ test statistic was also calculated to determine whether the prevalence of all PWUO and each opioid use group was significantly associated with period. The Rao-Scott $\chi^{2}$ test is a design-adjusted version of the Pearson $\chi^{2}$ test, with the same null hypothesis that there is no association between the row and column variables. ${ }^{196}$ Because both outcome variables were found to be significantly associated with period, weighted prevalence and standard errors were calculated for each survey year for all groups in order to upload into the NCI Joinpoint Trend software.

\section{Subaim 1.2: To ascertain the prevalence of all substance use in all PWUO as well as all opioid use groups and determine whether they differ over time.}

The period-stratified weighted prevalence of the self-reported use of all other core substances (pain relievers, heroin, alcohol, tranquilizers/sedatives, tobacco, marijuana, cocaine, crack, hallucinogens/inhalants, and stimulants) was calculated for all PWUO and opioid use groups. The reported weighted prevalence in the Results chapter is based on the sample size of each core substance category within each period. A Rao-Scott $\chi^{2}$ test statistic was also calculated to test whether the prevalence was significantly associated with period. Again, a post hoc analysis of each substance that was significantly associated with period for each outcome group (i.e., all PWUO; each opioid use group) was conducted using joinpoint regression to identify the point(s) in time where the linear trend of the weighted prevalence of each substance changed significantly. 


\section{Aim 2 analytic strategy}

Subaim 2.1: To identify the latent class variable of polydrug use in PWUO using the past month use variables of the 11 core substance indicators (pain relievers, heroin, heavy alcohol, tranquilizers/sedatives, cigarettes, other tobacco products, marijuana, cocaine, crack, hallucinogens/inhalants, and stimulants).

Latent class analysis was performed using Mplus version 8.2 using the recoded "past 30-day use" variables for each all 11 core substance indicators. It should be noted that the alcohol variable used in the latent class analysis model was limited to a recoded dichotomous (yes/no) dummy variable for heavy alcohol drinking, which included those who reported binge drinking in the past month. Figure 3.2 is a diagram of the relationship between the polydrug latent variable and the substance indicators used in this study.

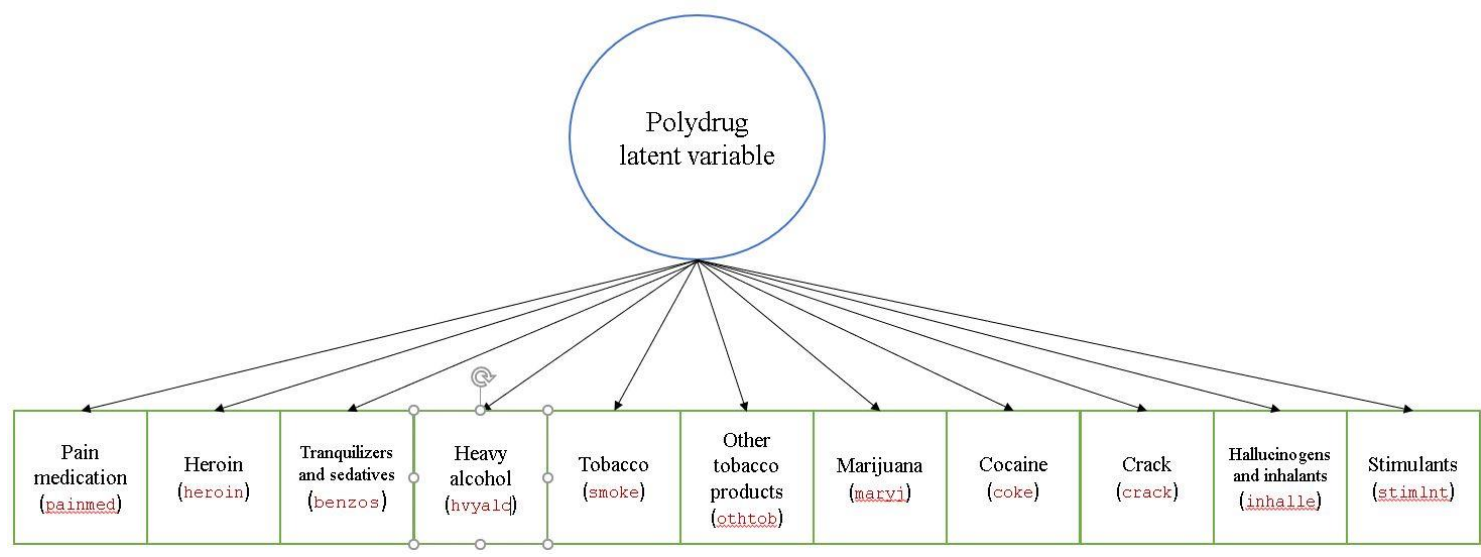

Figure 3.2 $\mid$ NSDUH core substance indicators included in the latent class analysis model 
To begin to model the latent variable, the number of classes specified in the Mplus code started at one, with the stepwise addition of classes to obtain fit statistics; the highest specified number of classes was eight. Also, to avoid the potential for estimation issues (e.g., singularities, local solutions), a high number of random starts was specified in the Mplus coding. The following established fit statistics and heuristics for LCA were utilized to guide the selection of the final number of latent classes:

- Akaike's Information Criteria (AIC)

- Bayes' Information Criteria (BIC)

- sample size adjusted Bayesian Information Criteria (ssBIC)

- entropy

Information criteria (IC) is used as a descriptive measure of comparing models in terms of relative fit and were developed to balance fit with parsimony. While there are a variety of ICs to choose from, the AIC, BIC, and ssBIC are commonly used for comparing competing models and differ only by the manner they penalize the likelihoodratio statistic $\left(G^{2}\right) .{ }^{194}$ LCA models with lower AIC and BIC values are deemed more parsimonious and are the preferred models.

Comparing entropy-based measures (e.g., a standardized entropy measure, $E$ ) is another strategy to determine the model with the best fit. The overall goal of using entropy-based measures is to determine whether the model produces classes that are well separated. ${ }^{197}$ Entropy values range from zero to one, with higher $E$ values indicating better latent class separation. ${ }^{197}$

Although the most commonly used method of determining the final class selection has been based on (in descending order) BIC, entropy, AIC, Lo-Mendell-Rubin 
Likelihood Ratio Test (LMR-LRT), ssBIC, adjusted LMR, and Bootstrapped Likelihood Ratio Test (BLRT), ${ }^{198}$ the selection of the final number of latent classes was ultimately determined using BIC and entropy values, and heavily guided by a priori theory as well as prevalence results from Aim 1. While LMR-LRT ${ }^{\$}$ has been used by previous LCA researchers to help determine the final number of classes, it was ultimately excluded because of the highly restrictive and uninterpretable results generated, likely due to the inclusion of the sampling weights. (Results of the LMR-LRT test for models without complex sample weights yielded significant p-values when testing the fit of up to eight classes). Additionally, specific guidance on final model selection for complex samples other than the use of BIC on a Mplus discussion page by Muthén and Muthén, could not be found in the literature.

Also, because many published studies have reported the use of an additional statistical software (e.g. SAS, STATA, SUDAAN, SPSS) when further analyzing the associations between polydrug latent classes (assessed in Mplus) with a multitude of independent variables of interest, ${ }^{19,99,199,200}$ it was decided that SAS version 9.4 will be used to carry out all further analyses. A .csv file was generated using Mplus, imported into SAS version 9.4 (Cary, NC, USA) after determining the final number of classes, and merged with the full SAS dataset matching by a created participant identification variable (id).

\footnotetext{
\#The LMR-LRT is a statistical test that compares the improvement in fit between neighboring class models (i.e., comparing $\mathrm{K}_{-1}$ and $\mathrm{K}$ class models), ultimately providing a $\mathrm{p}$-value that can be used to determine if the fit is significantly improved with the inclusion of an additional class. ${ }^{194}$
} 
Subaim 2.2: To determine whether the prevalence of each latent class has remained constant.

For this subaim, the Rao-Scott $\chi^{2}$ analysis was performed to test whether the weighted prevalence of the polydrug latent classes differed over time. Post hoc analysis was also conducted using the NCI joinpoint regression software to determine the year(s) where the linear trend of the weighted prevalence of each polydrug latent class may have significantly changed.

\section{Aim 3 analytic strategy}

The purpose of Aim 3 was to establish the associations between polydrug groups among PWUO and various set and setting correlates, but with a focus on latent classes that existed following Wave II of high opioid mortality in the U.S. ${ }^{10}$

Subaim 3.1: To determine the prevalence and bivariable associations of the following covariates using the latent class variable as an outcome: age, sex, race/ethnicity, total household income, education level, employment status, marital status, age at prescription pain reliever initiation, opioid use disorder, depression, health insurance status, past year substance use treatment, county type, and residential transience.

Prior to analyzing the prevalence and bivariable associations, LCA was used to model the polydrug latent classes among PWUO using data from 2010 to 2017; this was done not only to utilize the most current data on the reported core substance use among 
PWUO, but also to examine polydrug latent classes that existed after the start of the $2^{\text {nd }}$ wave of high opioid mortality. ${ }^{10}$ (However, it should be noted that patterns of substance use endorsement probabilities for the polydrug latent variable using the 2010 to 2017 data were very comparable to those enumerated for Aim 2). Using SAS 9.4, the prevalence of the following covariates was calculated for each latent polydrug class: age, sex, race/ethnicity, total household income, education level, employment status, marital status, age at prescription pain reliever initiation, opioid use disorder, depression, health insurance status, county type, and residential transience. It should be stressed that the correlates chosen for Aim 3 has been to be interconnected with one another, especially in the context of the opioid crisis. ${ }^{201}$ Finally, bivariate analyses using Rao-Scott $\chi^{2}$ tests were also performed to assess the associations between polydrug latent class membership and each of the set and setting variables listed above.

\section{Subaim 3.2: To determine the multivariable associations of specific combinations of variables using the drug, set, and setting model of substance use behavior to predict membership of the polydrug latent class.}

Because the basis of the drug, set, and setting framework suggests that all correlates are interconnected and affect a person's risk of substance use, a full multinomial logistic regression model with all covariates listed in Subaim 3.1 will be executed after testing for multicollinearity. The results of this logistic regression model were used to assess the previously mentioned hypotheses and sub-hypotheses, but are detailed again as follows: 
Hypothesis 1: Polydrug latent classes will differ by sociodemographic characteristics. Sub-hypothesis 1.1: The differences between the associations will be driven by the types and number of substances used within each Class, with the 'combination opioid polydrug Class' more likely than other Classes to be male, non-Hispanic white, unemployed, younger at time of survey, uninsured, and having less education and less total household income.

Hypothesis 2: Polydrug latent classes will differ by their opioid use history and OUD status.

Sub-hypothesis 2.1: The differences between the associations will be driven by the types and number of substances used within each Class, with the 'combination opioid polydrug Class' more likely than other Classes to have initiated prescription opioid use at an early age and to have opioid use disorder in the past year.

Hypothesis 3: Polydrug latent classes will differ by their environment. Sub-hypothesis 3.1: The differences between the associations will be driven by the types and number of substances used within each Class, with the 'combination opioid polydrug Class' more likely than other Classes to reside in a large metro and to have moved at least twice in the past year. 


\section{Deciding on the final number of latent classes}

Statistical power is the probability that the null hypothesis is correctly rejected when the alternative hypothesis is true. In traditional epidemiologic studies, not only is power affected by study design, it may be calculated using a number of values such as sample size, percent confidence level, ratio of unexposed to exposed (or ratio of controls to cases in case-control studies), and effect size. However, when discussing sample size and power in studies that involve LCA, the concern centers around obtaining the correct number of classes in the latent variable, rather than an effect size for a population (e.g., relative risk). Therefore, the null and alternative hypotheses for power tests in LCA are as follows:

$\mathrm{H}_{0}$ : The true number of latent classes is $\mathrm{K}_{-1}$.

H1: The true number of latent classes is $\mathrm{K}$.

Statistical power in LCA requires multiple simulations to be conducted for each fit statistic (i.e., AIC, BIC, ssBIC, etc.), to discern whether a particular fit statistic would be able to reject the $\mathrm{H}_{0}$. Ideally, to determine the exact power for this study, simulations should also be completed; unfortunately, this could not be feasibly done. Therefore, provided in this section are the power results of a Monte Carlo simulation study

conducted by Nylund et al. (see Figure 3.3). ${ }^{202}$ 


\begin{tabular}{|c|c|c|c|c|c|c|c|c|c|c|c|c|c|c|c|c|c|c|c|c|c|}
\hline \multirow[b]{4}{*}{ Model } & \multirow[b]{4}{*}{$n$} & \multicolumn{20}{|c|}{ Latent Class Analysis with Categorical Outcomes } \\
\hline & & \multicolumn{5}{|c|}{$A I C$} & \multicolumn{5}{|c|}{ CAIC } & \multicolumn{5}{|c|}{$B I C$} & \multicolumn{5}{|c|}{ Adjusted BIC } \\
\hline & & \multicolumn{5}{|c|}{ Classes } & \multicolumn{5}{|c|}{ Classes } & \multicolumn{5}{|c|}{ Classes } & \multicolumn{5}{|c|}{ Classes } \\
\hline & & 2 & 3 & 4 & 5 & 6 & 2 & 3 & 4 & 5 & 6 & 2 & 3 & 4 & 5 & 6 & 2 & 3 & 4 & 5 & 6 \\
\hline \multirow{5}{*}{$\begin{array}{l}\text { 8-item } \\
\text { (Simple, } \\
\text { Equal) }\end{array}$} & 200 & 0 & 0 & 75 & 22 & 3 & 0 & 5 & 95 & 0 & 0 & 0 & 1 & 99 & 0 & 0 & 0 & 0 & 83 & 15 & 2 \\
\hline & 500 & 0 & 0 & 68 & 27 & 5 & 0 & 0 & 100 & 0 & 0 & 0 & 0 & 100 & 0 & 0 & 0 & 0 & 100 & 0 & 0 \\
\hline & 1000 & 0 & 0 & 60 & 32 & 8 & 0 & 0 & 100 & 0 & 0 & 0 & 0 & 100 & 0 & 0 & 0 & 0 & 100 & 0 & 0 \\
\hline & & \multicolumn{5}{|c|}{ Classes } & \multicolumn{5}{|c|}{ Classes } & \multicolumn{5}{|c|}{ Classes } & \multicolumn{5}{|c|}{ Classes } \\
\hline & $n$ & 2 & 3 & 4 & 5 & 6 & 2 & 3 & 4 & 5 & 6 & 2 & 3 & 4 & 5 & 6 & 2 & 3 & 4 & 5 & 6 \\
\hline \multirow{5}{*}{$\begin{array}{l}\text { 8-item } \\
\text { (Simple, } \\
\text { Unequal) }\end{array}$} & 200 & 0 & 26 & & 24 & 2 & 83 & 17 & 0 & 0 & 0 & 58 & 42 & 0 & 0 & 0 & 0 & 29 & 58 & 12 & 1 \\
\hline & 500 & 0 & 2 & 67 & 27 & 4 & 6 & 83 & 11 & 0 & 0 & 1 & 72 & 27 & 0 & 0 & 0 & 12 & 88 & 0 & 0 \\
\hline & 1000 & 0 & 0 & 62 & 33 & 5 & 0 & 26 & 74 & 0 & 0 & 0 & 12 & 88 & 0 & 0 & 0 & 0 & 100 & 0 & 0 \\
\hline & & \multicolumn{5}{|c|}{ Classes } & \multicolumn{5}{|c|}{ Classes } & \multicolumn{5}{|c|}{ Cinosos } & & & tosose & & \\
\hline & $n$ & 2 & 3 & 4 & 5 & 6 & 2 & 3 & 4 & 5 & 6 & 2 & 3 & 4 & 5 & 6 & 2 & 3 & 4 & 5 & 6 \\
\hline \multirow{5}{*}{$\begin{array}{l}\text { 15-item } \\
\text { (Simple, } \\
\text { Equal) }\end{array}$} & 200 & 0 & 43 & 41 & 13 & 3 & 0 & 100 & 0 & 0 & 0 & 0 & 100 & 0 & 0 & 0 & 0 & 62 & 31 & 6 & 1 \\
\hline & 500 & 0 & 32 & 41 & 17 & 6 & 0 & 100 & 0 & 0 & 0 & 0 & 100 & 0 & 0 & 0 & 0 & 99 & 1 & 0 & 0 \\
\hline & 1000 & 0 & 31 & 45 & 17 & 6 & 0 & 100 & 0 & 0 & 0 & 0 & 100 & 0 & 0 & 0 & 0 & 100 & 0 & 0 & 0 \\
\hline & & \multicolumn{5}{|c|}{ Classes } & \multicolumn{5}{|c|}{ Classes } & \multicolumn{5}{|c|}{ Classes } & \multicolumn{5}{|c|}{ Classes } \\
\hline & $n$ & 2 & 3 & 4 & 5 & 6 & 2 & 3 & 4 & 5 & 6 & 2 & 3 & 4 & 5 & 6 & 2 & 3 & 4 & 5 & 6 \\
\hline \multirow{3}{*}{$\begin{array}{l}\text { 10-item } \\
\text { (Complex, } \\
\text { Unequal) }\end{array}$} & 200 & 0 & 5 & 67 & 24 & 3 & 2 & 97 & 1 & 0 & 0 & 0 & 92 & 8 & 0 & 0 & 0 & 7 & 76 & 15 & 2 \\
\hline & 500 & 0 & 0 & 55 & 35 & 9 & 0 & 44 & 56 & 0 & 0 & 0 & 24 & 76 & 0 & 0 & 0 & 1 & 98 & 1 & 0 \\
\hline & 1000 & 0 & 0 & 46 & 39 & 14 & 0 & 1 & 99 & 0 & 0 & 0 & 0 & 100 & 0 & 0 & 0 & 0 & 100 & 0 & 0 \\
\hline
\end{tabular}

Figure 3.3 | Percentage of Times the Lowest Value Occurred in Each Class Model for the AIC, CAIC, BIC, and Adjusted BIC ${ }^{202}$

In this current study, we determined that the best fit model had a somewhat simple structure (i.e., models with item probabilities that are particularly high or low for a given class), with four classes of unequal sizes using 11 items, and over 16,000 subjects. As shown in Figure 3.3, BIC and ssBIC were the best indicators to determine the most parsimonious number of latent classes given the structure of the final model, suggesting that the current sample size is sufficient to use BIC or ssBIC to determine final class sizes. $^{202}$ It should also be noted that while missing data may pose an issue to the validity of the results, Mplus can factor in missing variables through a simple Missing are command. Once missing values are excluded, Mplus uses all available data to estimate the model using full information maximum likelihood (FIML), which assumes that data are missing at random (MAR). ${ }^{203}$ 


\section{CHAPTER IV}

RESULTS

\section{Aim 1 Results}

Subaim 1.1: To ascertain the prevalence of all PWUO and the opioid use groups (i.e., heroin only, prescription opioid only, and combination opioids) and determine whether they differ over time.

\section{Prevalence among PWUO}

From 2002 to 2017 , a total of 621,571 individuals participated in the NSDUH survey. Of these, the weighted prevalence of respondents $(n=16,985)$ reported having used either heroin or prescription pain relievers was $1.78 \%(\mathrm{SE}=0.042)$. Overall sample sizes with their respective weighted prevalence rate (PR) and standard errors (SE) by past month opioid use status and period are shown in Table 4.1. Overall, individuals reporting past month opioid use were the vast minority in the U.S., with the highest PR occurring in period 2 (i.e., 2006 to 2009) [PR (SE): 2.01\% (0.49)] and lowest in period 4 (i.e., 2014 to 2017) [PR (SE): 1.48\% (0.58)]. 


\section{The longitudinal trend of PWUO prevalence}

Also shown in Table 4.1 are the results of the bivariable analysis of the

prevalence of opioid use and period using a Rao-Scott Chi-square $\left(\chi^{2}\right)$ test. Based on the results, there was a significant association between the weighted prevalence of PWUO and period $\left[\chi^{2}(\mathrm{df}),(\mathrm{p}\right.$-value): $58.30(3),(<0.0001)]$. Examining the change in prevalence visually, the weighted prevalence of PWUO increases from period 1 to 2 but decreases in subsequent periods, as shown in Figure 4.1. Joinpoint regression was performed to provide a quantitative examination of prevalence trends of past month PWUO. While the full results of the joinpoint regression may be found in Supplemental Table (ST) 4.1 in Appendix A, the main results of this analysis are presented in Figure 4.2. Joinpoint analysis established two time-segments that had statistically significant slopes (m): 1) 2002 to 2010 [m (p-value): 0.028 (0.020)], and 2) 2010 to 2017 [m (pvalue): $-0.117(<0.001)]$. Time-segment 1 had a significantly positive increase in the weighted prevalence of past month PWUO, while time-segment 2 showed a significant decrease in the trajectory of the weighted prevalence. 


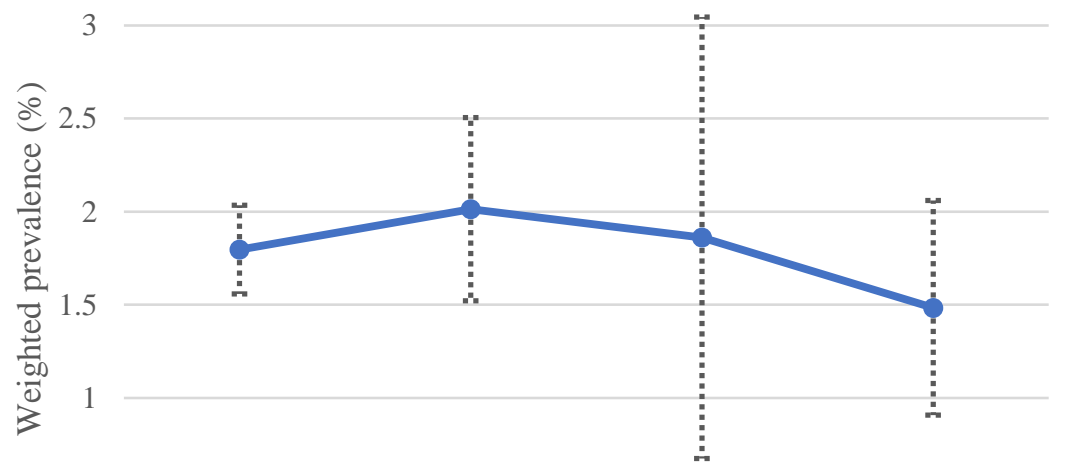

0.5

0
(1) 2002 to 2005
(2) 2006 to 2009
(3) 2010 to 2013
(4) 2014 to 2017

Period

Figure 4.1 | Weighted prevalence of people who reported past month use of opioids by period $(\mathrm{n}=16,985)$, NSDUH 2002 to 2017

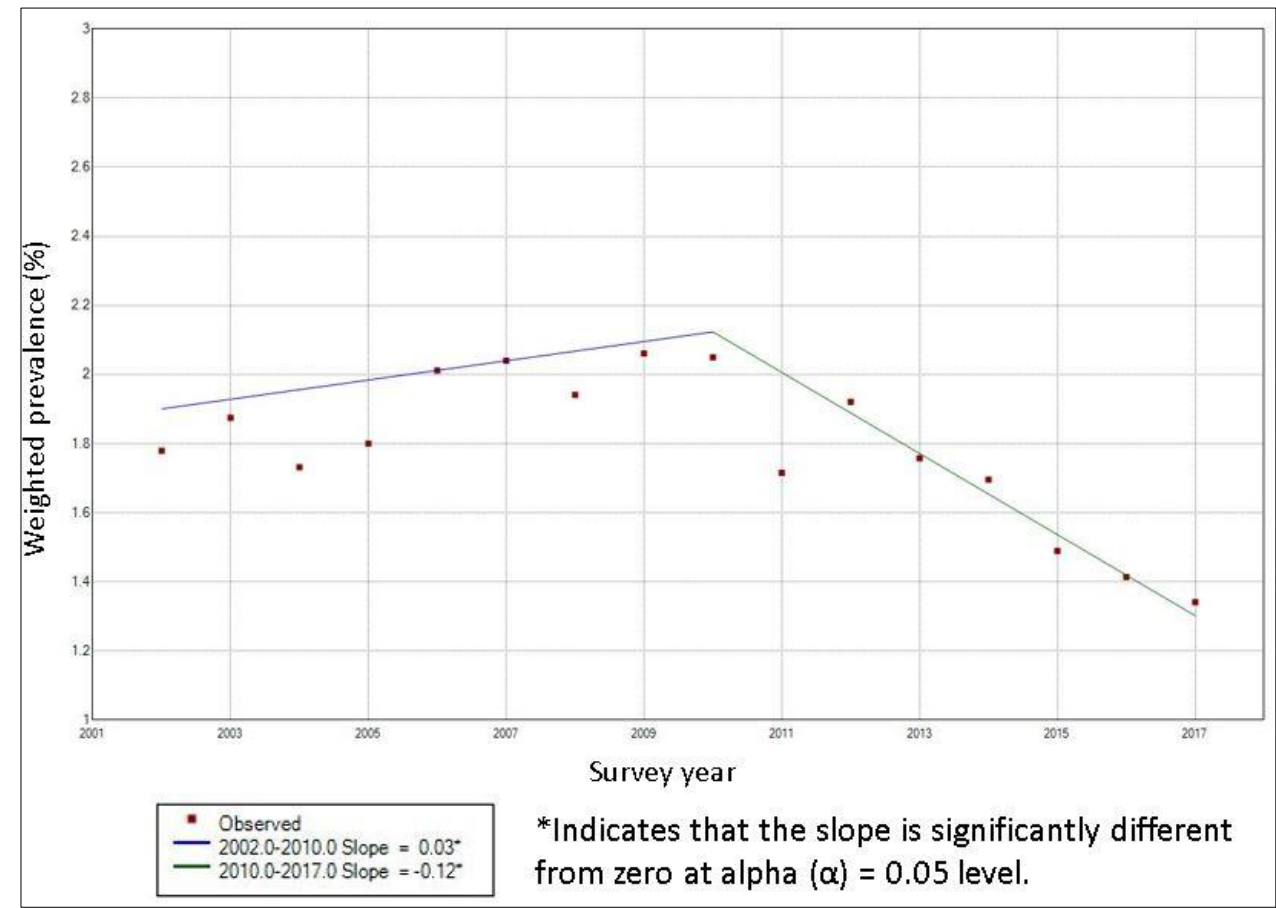

Figure 4.2 | Joinpoint analysis of the weighted prevalence of PWUO $(n=16,985)$ 
Table 4.1 | Weighted prevalence of all NSDUH participants by opioid use status and period (N =621,571), NSDUH 2002 to $2017^{\mathrm{a}, \mathrm{b}}$

\begin{tabular}{|c|c|c|c|c|c|c|c|c|c|c|c|c|c|c|}
\hline \multirow{3}{*}{$\begin{array}{c}\text { NSDUH } \\
\text { Participants }\end{array}$} & \multicolumn{12}{|c|}{ Period } & \multicolumn{2}{|c|}{$\begin{array}{c}\text { Rao-Scott } \\
\text { analysis }\end{array}$} \\
\hline & \multicolumn{3}{|c|}{ (1) 2002 to 2005} & \multicolumn{3}{|c|}{ (2) 2006 to 2009} & \multicolumn{3}{|c|}{ (3) 2010 to 2013} & \multicolumn{3}{|c|}{ (4) 2014 to 2017} & $\begin{array}{l}\chi^{2} \\
(\mathrm{df})\end{array}$ & $\begin{array}{c}\text { p- } \\
\text { value }\end{array}$ \\
\hline & $\mathrm{n}$ & $\%$ & SE & $\mathrm{n}$ & $\%$ & SE & $\mathrm{n}$ & $\%$ & SE & $\mathrm{n}$ & $\%$ & SE & & \\
\hline $\begin{array}{c}\text { Reported } \\
\text { use of } \\
\text { opioids in } \\
\text { past month } \\
(\mathrm{n}=16,985)\end{array}$ & 4,457 & 1.80 & 0.24 & 4,987 & 2.01 & 0.49 & 4,359 & 1.86 & 1.19 & 3,182 & 1.48 & 0.58 & 5830 & \\
\hline $\begin{array}{c}\text { Did not } \\
\text { report use } \\
\text { of opioids } \\
\text { in past } \\
\text { month } \\
(\mathrm{n}= \\
604,586)\end{array}$ & 143,474 & 98.20 & 0.04 & 144,897 & 97.99 & 0.13 & 148,986 & 98.14 & 0.12 & 167,229 & 98.52 & 0.10 & (3) & 0.0001 \\
\hline
\end{tabular}

Abbreviations: $\mathrm{n}=$ total number of survey respondents; $\%=$ weighted column percentage; $\mathrm{SE}=$ standard error; $\chi^{2}(\mathrm{df})=$ Chi-square statistic (degrees of freedom)

Note: $95 \%$ confidence intervals for all reported prevalence indicated reliable measures

Note: Bolded $\chi^{2}$ and $p$-value denotes statistical significance at $p<0.05$.

${ }^{a}$ Weighted prevalence was based on the total number of survey participants within each period

${ }^{b}$ PWUO were defined as participants who had reported that they had used heroin in the past month; had nonmedically used prescription pain relievers in the past month (in NSDUH 2002-2014), or misused prescription pain relievers in the past month (in NSDUH 2015-2017) 


\section{Prevalence among opioid use groups}

After stratifying the entire population of PWUO into three past month opioid use

groups (i.e., PPR-only, heroin-only, and combination opioid), it was clear that the number and proportion of individuals who reported past month PPR-only use vastly exceeded those in the heroin-only and combination opioid use groups. The overall weighted prevalence and standard errors for each opioid use group were: past month PPR-only [PR (SE) $93.36 \%(\mathrm{SE}=0.11)$ ], heroin-only [PR $(\mathrm{SE})=3.61 \%(0.16)]$, and combination opioid use groups $[\mathrm{PR}(\mathrm{SE})=3.03 \%(0.12)]$.

Table 4.2 shows the weighted prevalence of the opioid use groups by period. (Because the focus was on PWUO, individuals who did not report past month opioid use were excluded from prevalence measures). Those who reported the use of only PPRs in the past month composed the majority with the highest weighted prevalence in period 1 [PR (SE): 96.46 (0.32)], and the lowest prevalence in period 4 [PR (SE): $87.51 \%$ (0.021)]. Conversely, the weighted prevalence of the past month heroin-only use group was highest in period 4 [PR (SE): 7.64\% (0.38)], and lowest in period 1 [PR (SE): $1.27 \%(0.17)]$. The prevalence of the past month combination opioid use group was also highest in period 4 [PR (SE): 96.46\% (0.32)], and the lowest in period 1 [PR (SE): $96.46 \%(0.32)]$. 
Table 4.2 | Weighted prevalence of past month opioid use categories among PWUO by period ( $\mathrm{n}=16,985)$, NSDUH 2002 to $2017^{\mathrm{a}, \mathrm{b}, \mathrm{c}, \mathrm{d}}$

\begin{tabular}{|c|c|c|c|c|c|c|c|c|c|c|c|c|c|c|}
\hline \multirow{3}{*}{$\begin{array}{l}\text { Opioid use } \\
\text { group }\end{array}$} & \multicolumn{12}{|c|}{ Period } & \multicolumn{2}{|c|}{$\begin{array}{l}\text { Rao-Scott } \\
\text { analysis }\end{array}$} \\
\hline & \multicolumn{3}{|c|}{ (1) 2002 to 2005} & \multicolumn{3}{|c|}{ (2) 2006 to 2009} & \multicolumn{3}{|c|}{ (3) 2010 to 2013} & \multicolumn{3}{|c|}{ (4) 2014 to 2017} & \multirow{2}{*}{$\chi^{2}(\mathrm{df})$} & \multirow{2}{*}{ p-value } \\
\hline & $\mathrm{n}$ & $\%$ & SE & $\mathrm{n}$ & $\%$ & SE & $\mathrm{n}$ & $\%$ & SE & $\mathrm{n}$ & $\%$ & SE & & \\
\hline $\begin{array}{c}\text { PPR-only } \\
(\mathrm{n}=15,897)\end{array}$ & $\begin{array}{c}430 \\
6\end{array}$ & $\begin{array}{c}96.4 \\
6\end{array}$ & 0.32 & 4770 & $\begin{array}{c}95.1 \\
0\end{array}$ & 0.038 & $\begin{array}{c}405 \\
9\end{array}$ & $\begin{array}{c}93.6 \\
8\end{array}$ & 0.13 & $\begin{array}{c}276 \\
2\end{array}$ & 87.51 & 0.021 & \multirow{3}{*}{$\begin{array}{l}134.7 \\
9(6)\end{array}$} & \multirow{3}{*}{$<0.0001$} \\
\hline $\begin{array}{l}\text { Heroin only } \\
\quad(\mathrm{n}=553)\end{array}$ & 80 & 2.27 & 0.15 & 88 & 2.31 & 0.25 & 144 & 2.78 & 0.66 & 241 & 7.64 & 0.377 & & \\
\hline $\begin{array}{c}\text { Combinatio } \\
\text { n opioid } \\
(\mathrm{n}=535)\end{array}$ & 71 & 1.27 & 0.17 & 129 & 2.59 & 0.28 & 156 & 3.54 & 0.54 & 179 & 4.86 & 0.356 & & \\
\hline
\end{tabular}

$\infty$ Abbreviations: $\mathrm{n}=$ total number of survey respondents; $\%=$ weighted column percentage; $\mathrm{SE}=$ standard error; $\chi^{2}(\mathrm{df})=\mathrm{Chi}$-square statistic (degrees of freedom)

Note: Bolded $\chi^{2}$ and $p$-value denote statistical significance at $\mathrm{p}<0.05$.

${ }^{a}$ All weighted prevalence were based on the total number of individuals for all opioid use groups within each period

bPPR use was defined as participants who had reported that they had nonmedically used or misused any PPR in the past month and did not heroin in the past month.

${ }^{\mathrm{c}}$ Heroin use was defined as participants who had reported that they had used heroin in the past month and did not nonmedically use or misuse PPRs in the past month.

${ }^{\mathrm{d} C o m b i n a t i o n}$ opioid use was defined as participants who had reported that they had used both heroin in the past month, as well as nonmedically used or misused PPRs in the past month. 


\section{The longitudinal trend of opioid use group prevalence}

Also shown in Table 4.2 are the results of the bivariable association between

opioid use group prevalence and period, again using the Rao-Scott $\chi^{2}$ test. The results of this test indicated that there was a significant association between the prevalence of all opioid use groups and period [ $\chi^{2}(\mathrm{df}),(\mathrm{p}$-value): $134.79(6),(<0.0001)]$. As shown in Figures 4.3 and 4.4, the overall weighted prevalence of those who reported past month PPR-only use has been decreasing since period 1, while the overall weighted prevalence of the past month heroin-only and combination opioid use groups had been increasing since period 1.

Joinpoint regression was also performed, and the main results of this analysis are presented in Figures 4.5 through 4.7. The prevalence of participants who reported having used only PPRs in the past month significantly decreased from 2002 to 2017 [m (p-value): -0.45 (0.0004)]. For the past month heroin-only use group, a significant change in the prevalence trend was detected from 2010 to 2017 [m (p-value): 1.062 (< 0.001)]. Among the past month combination opioid use group, no joinpoint was found, but there was an overall positive prevalence trend from 2002 to 2017 [m (p-value): 0.35 (0.013)]. (For all results of the joinpoint analyses in this section, see ST 4.2 through 4.4 in Appendix A). 


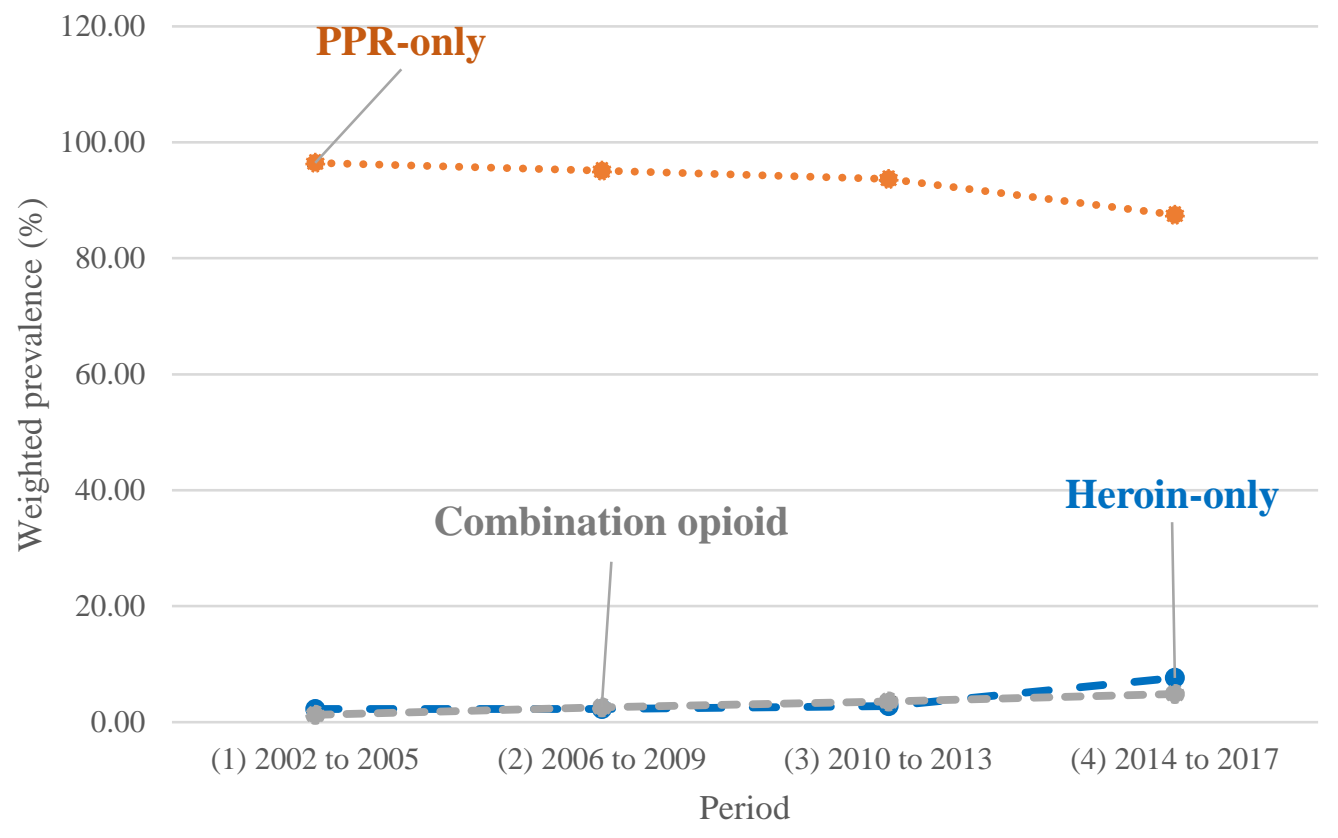

Figure 4.3 $\mid$ Weighted prevalence of all past-month opioid use categories among PWUO by period $(\mathrm{n}=16,985)$, NSDUH 2002 to 2017

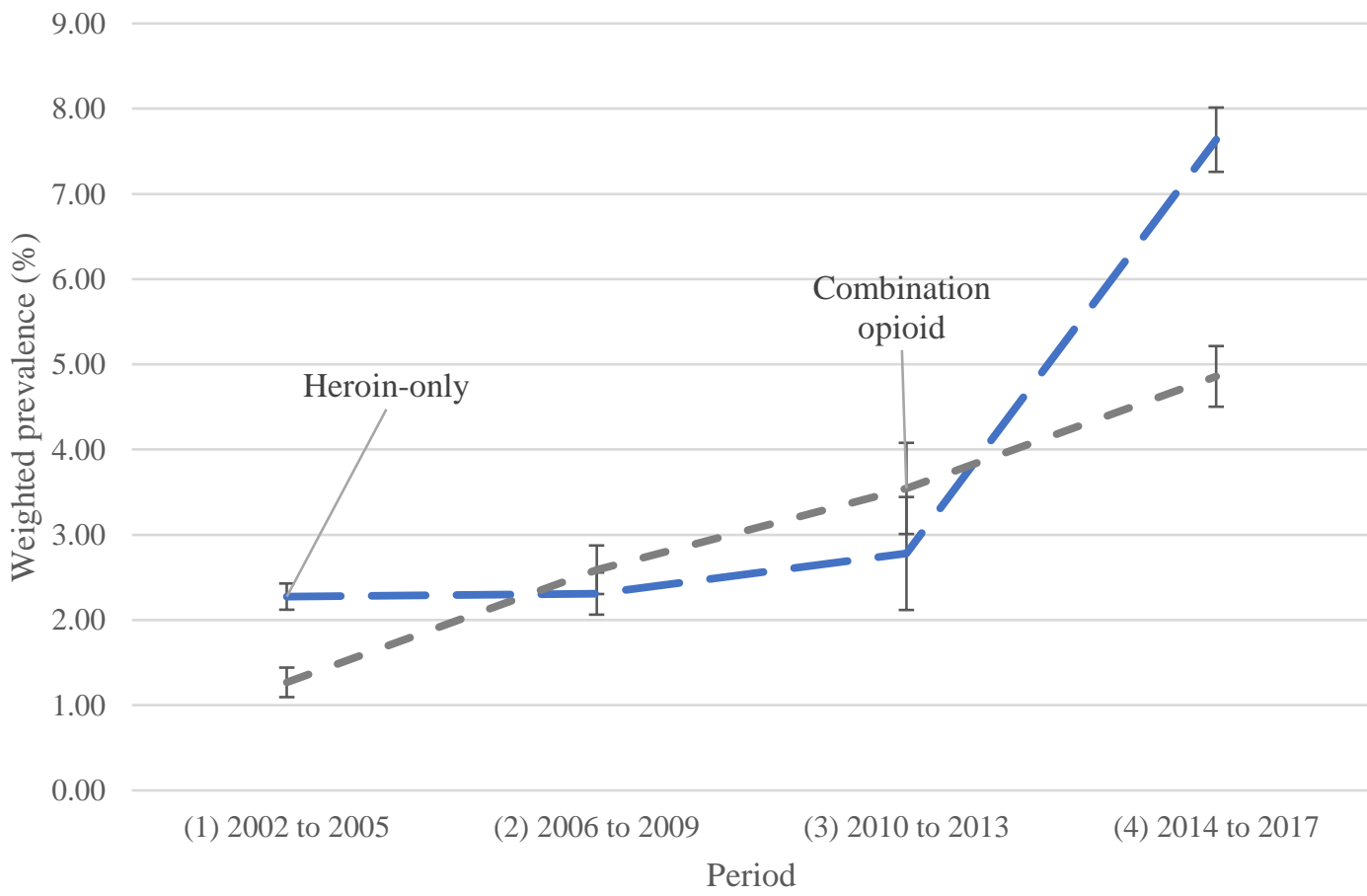

Figure 4.4 | Weighted prevalence of past-month heroin only and combination opioid use groups among PWUO by period ( $\mathrm{n}=1,088)$, NSDUH 2002 to 2017 


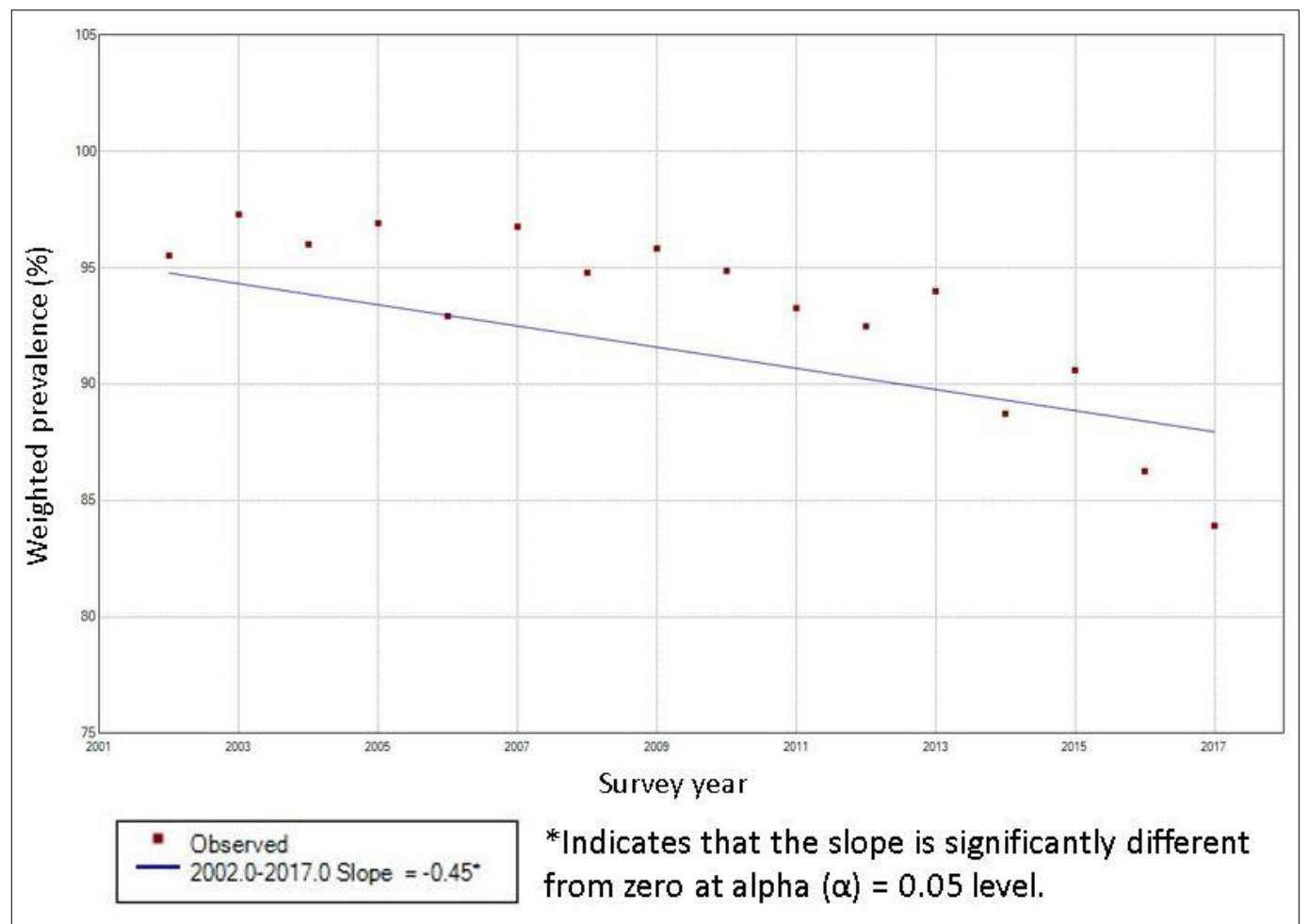

Figure 4.5 $\mid$ Joinpoint analysis of the weighted prevalence of past month PPR-only use group $(\mathrm{n}=15,897)$

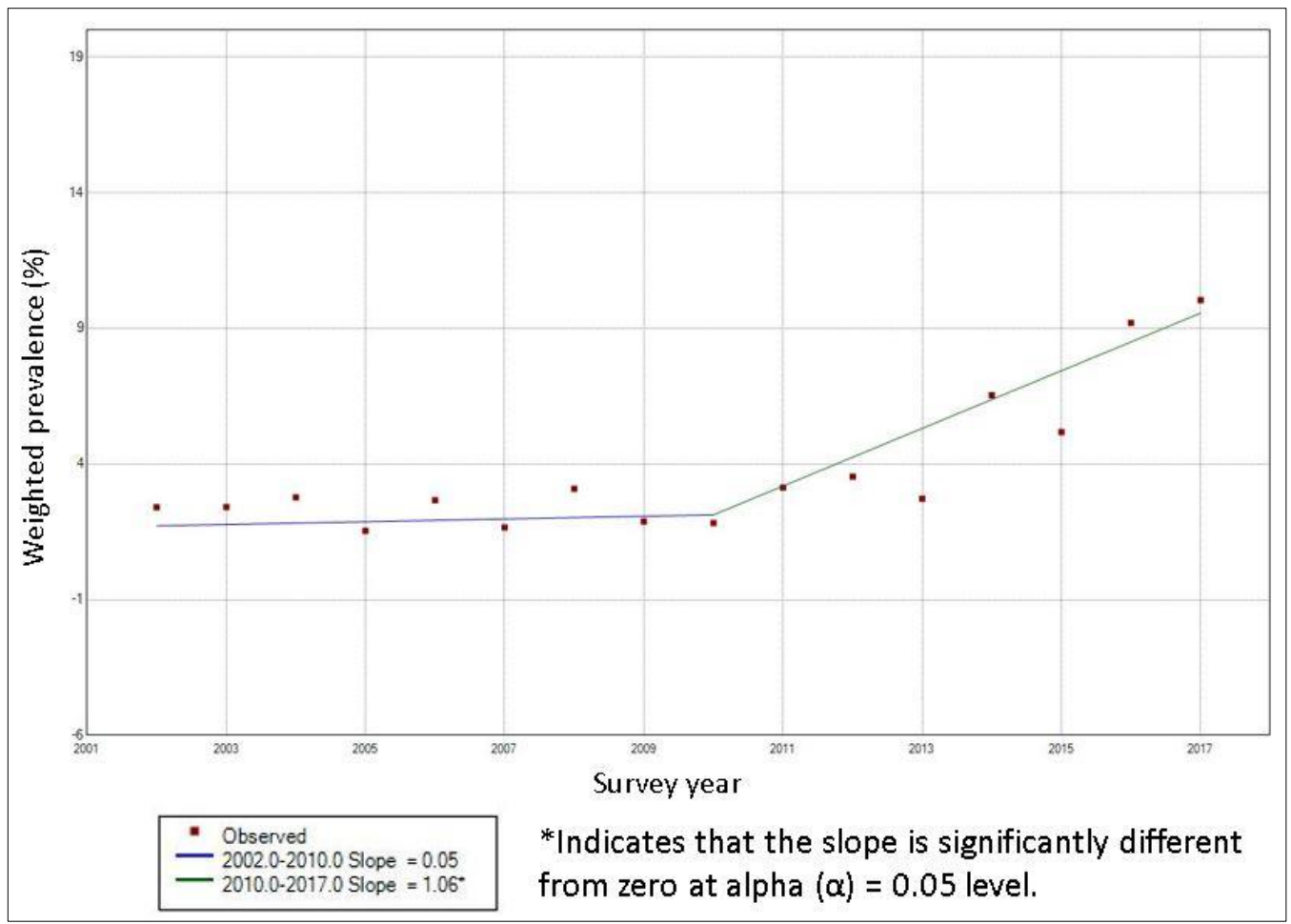

Figure 4.6 | Joinpoint analysis of the weighted prevalence of past month heroin-only use group $(\mathrm{n}=553)$ 


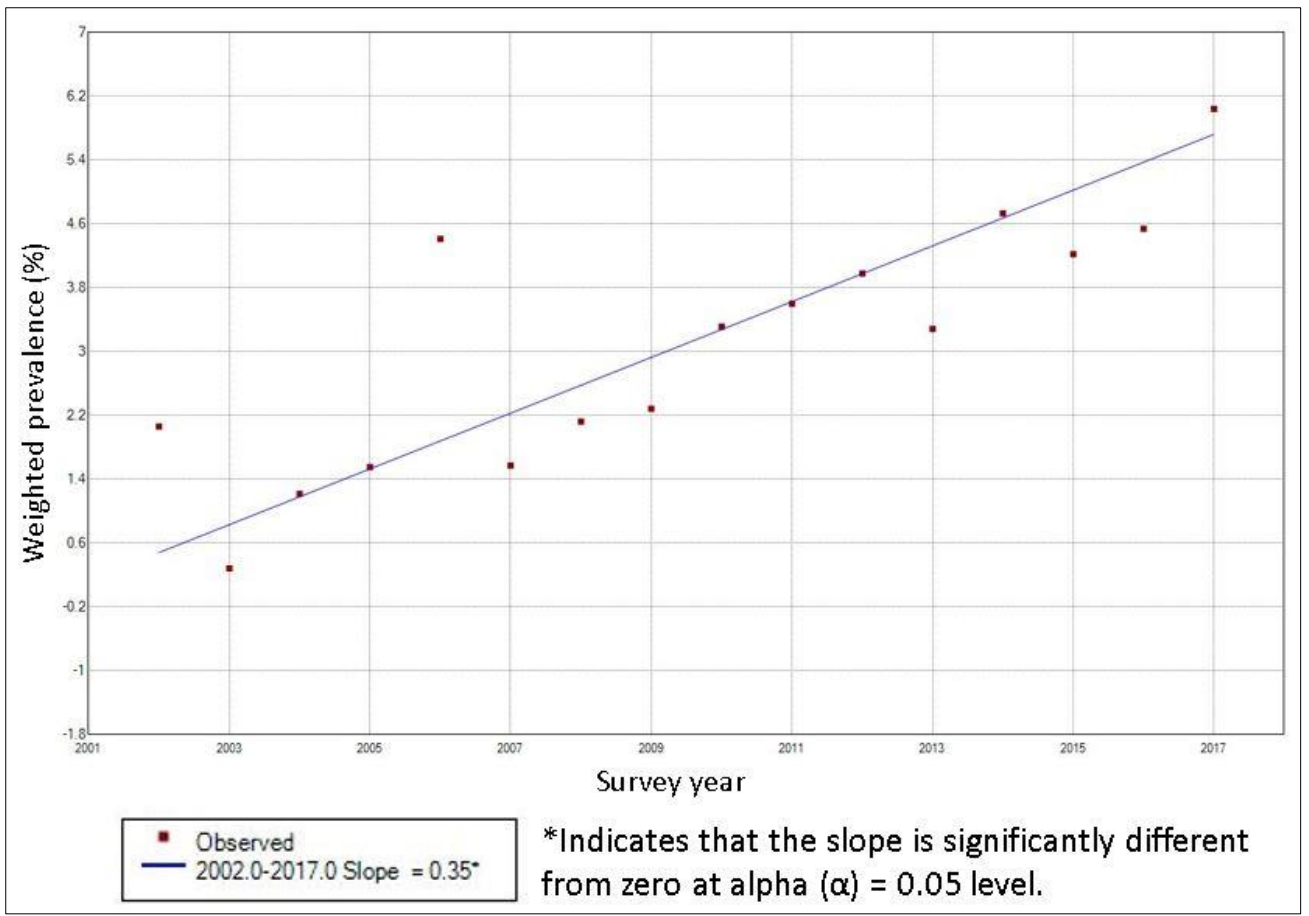

Figure 4.7 | Joinpoint analysis of the weighted prevalence of past month combination opioid use group $(\mathrm{n}=535)$ 
Subaim 1.2: To ascertain the prevalence of all substance use in all PWUO as well as all opioid use groups and determine whether they differ over time

\section{Prevalence of substance use among all PWUO}

As shown in Figure 4.8, the core substance most frequently reported as having been used among PWUO was cigarettes, followed by marijuana and binge alcohol. The weighted prevalence of illicit substance use (i.e., cocaine, crack, hallucinogens/inhalants) and prescription stimulants were the lowest among PWUO. (For full overall prevalence results, see ST 4.5 in Appendix A).

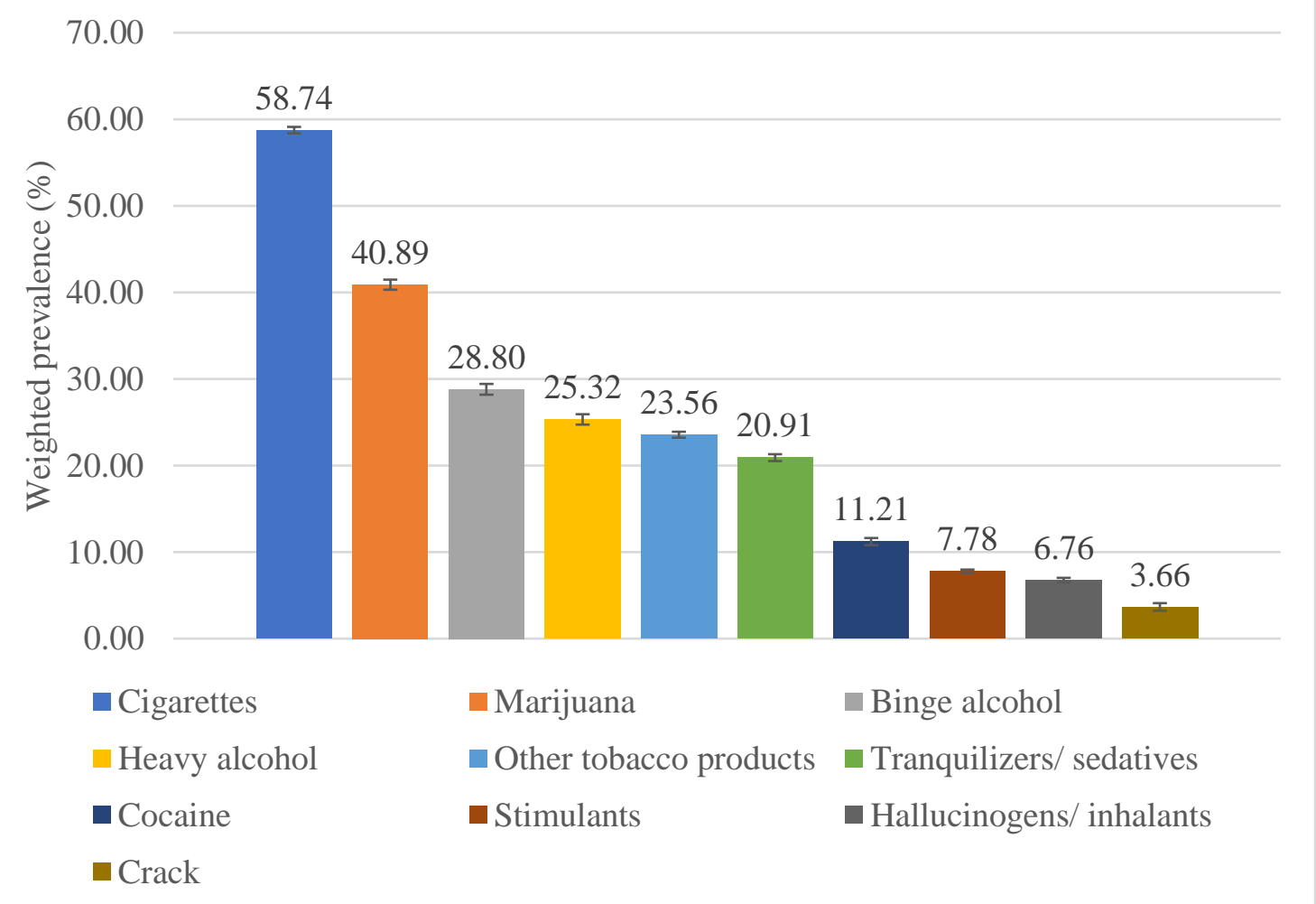

Figure 4.8 Overall weighted prevalence of past month core substance use among PWUO $(\mathrm{n}=16,985)$, NSDUH 2002 to 2017 


\section{Longitudinal trends of substance use among PWUO}

The prevalence of reported core substance use among PWUO is shown in Figures 4.9, 4.10, and 4.11. (For the full table of results, see ST 4.6 in Appendix A). It should be noted that Figures 4.9 through 4.11 break down the core substances into three groups:

a. Past month binge or heavy alcohol use

b. Past month cigarette smoking, other tobacco product use, and marijuana smoking

c. Past month prescription tranquilizers/sedatives, prescription stimulants, cocaine, crack, and hallucinogen/inhalant use

The weighted prevalence of reported binge and heavy alcohol drinking was shown to have declined after period 2. Table 4.3 shows the bivariable analysis results for the association of core substance prevalence and period among PWUO. The weighted prevalence of core substances that differed significantly over time for all PWUO were heavy alcohol, cigarettes, other tobacco products, hallucinogens and inhalants, and stimulants use.

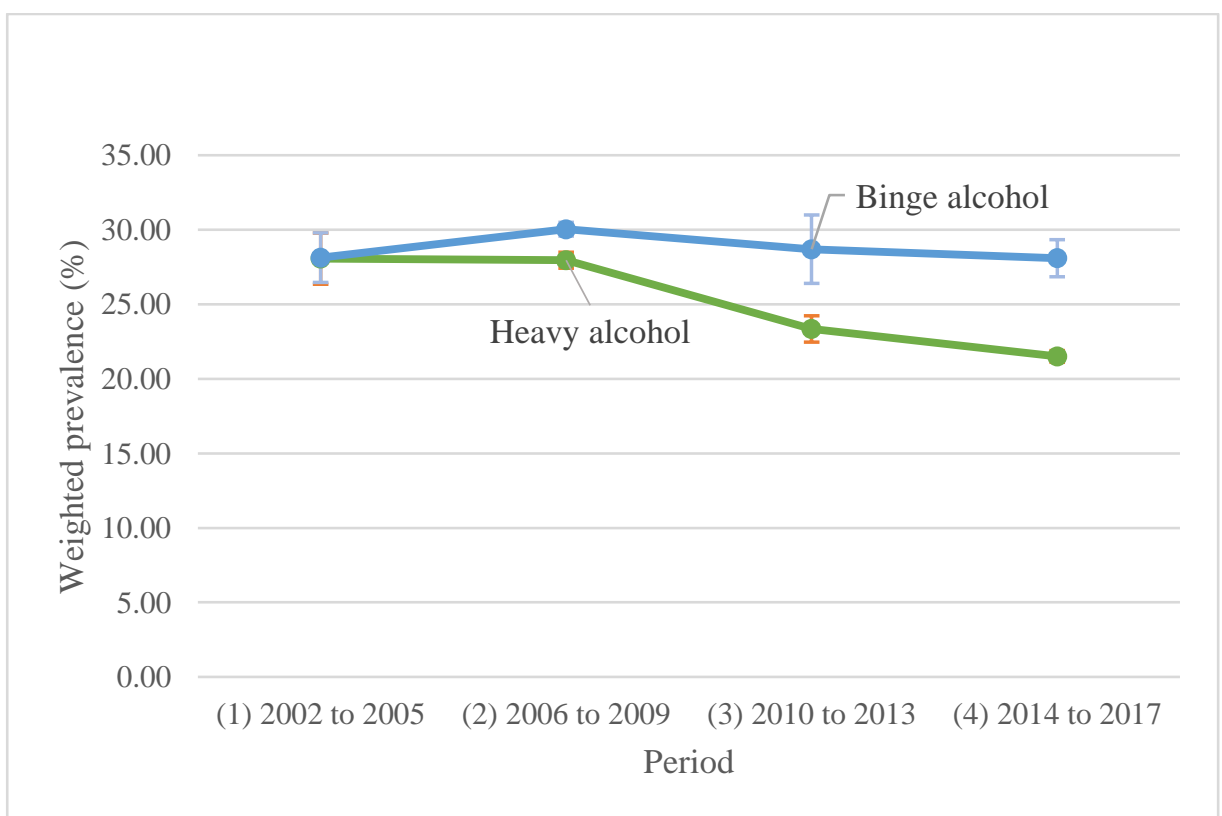

Figure 4.9 $\mid$ Weighted prevalence of past month heavy and binge alcohol drinking among past month PWUO by period ( $\mathrm{n}=16,985)$, NSDUH 2002 to 2017 


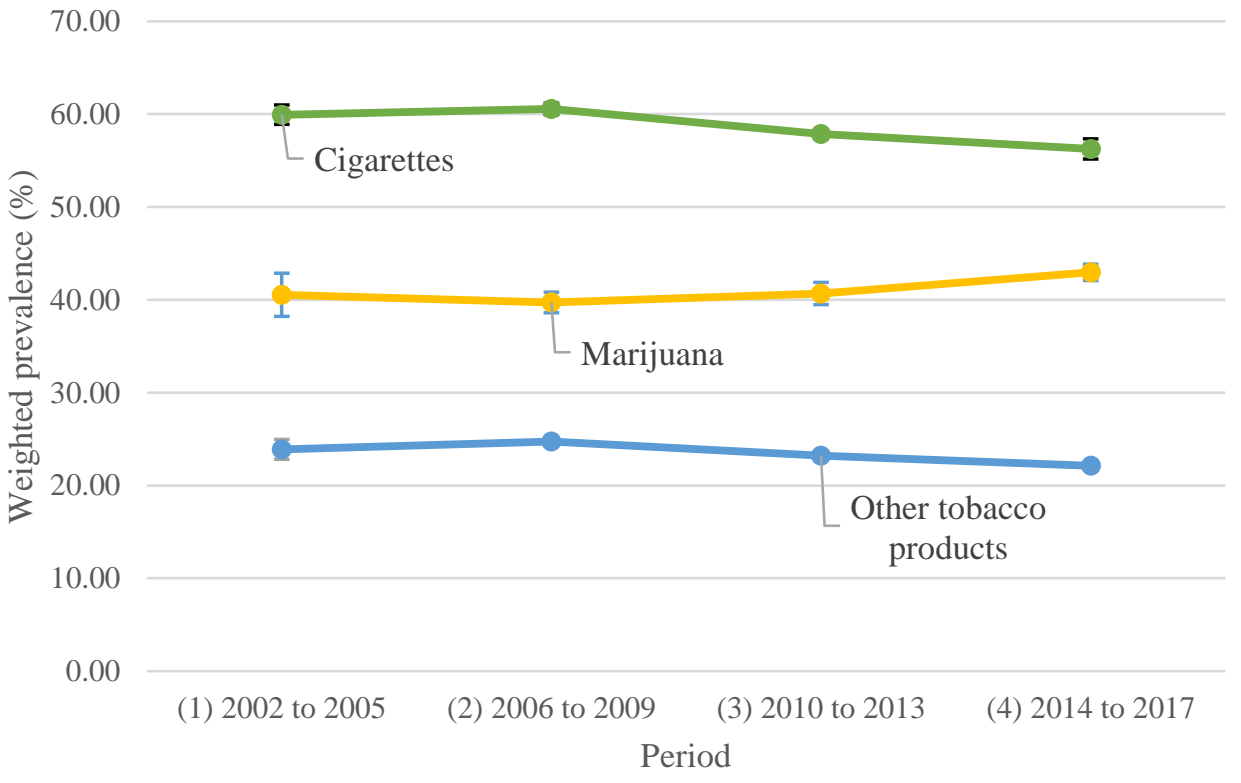

Figure 4.10 | Weighted prevalence of reported past month cigarette smoking, other tobacco use, and marijuana use among past month PWUO by period $(n=16,985)$, NSDUH 2002 to 2017

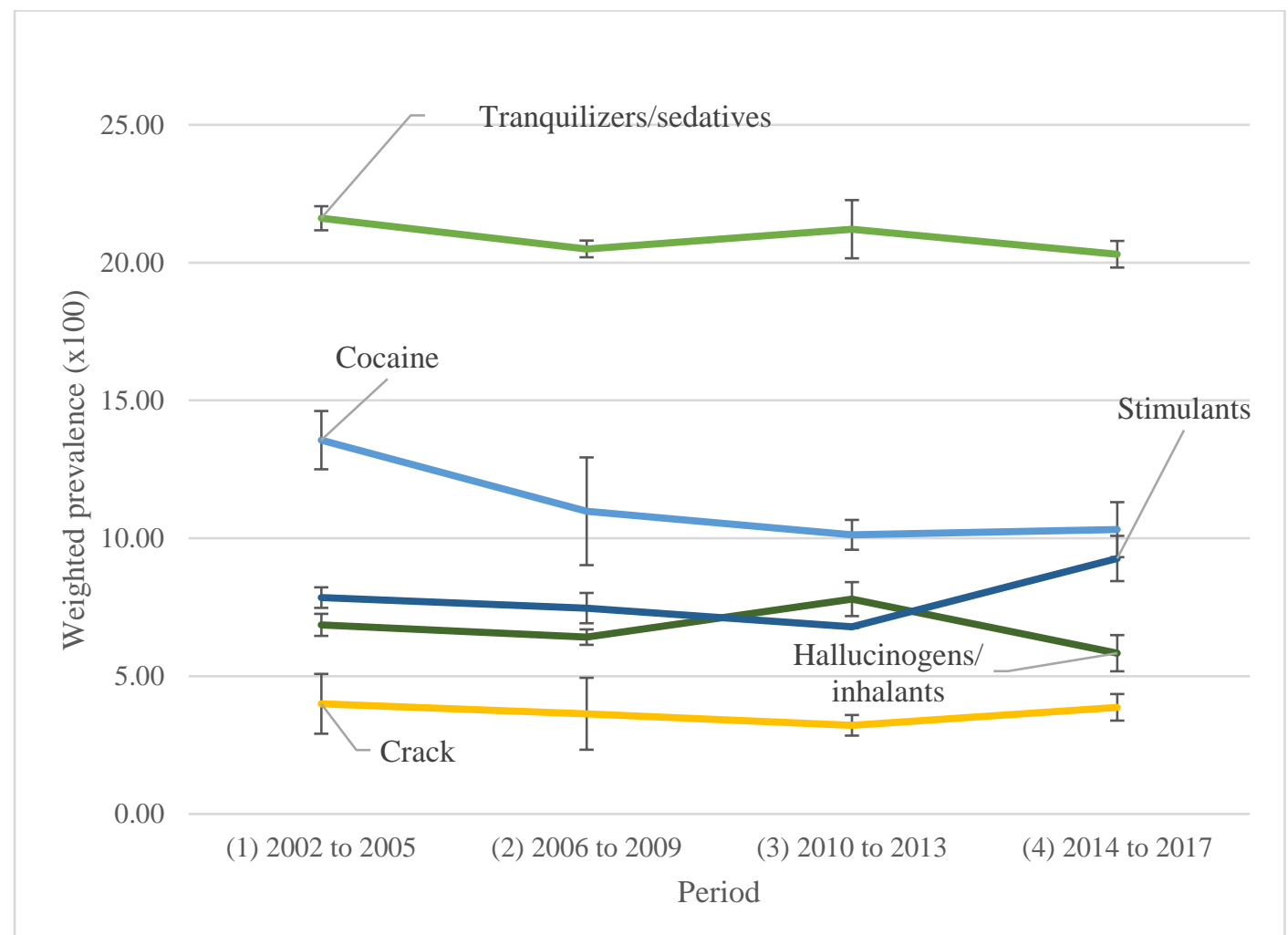

Figure 4.11 | Weighted prevalence of reported past month tranquilizer/sedative, cocaine, crack, hallucinogen/inhalant, and stimulant use among past month PWUO by period $(n=16,985)$,

NSDUH 2002 to 2017 
Table 4.3 Rao-Scott $\chi^{2}$ analyses for the association of prevalence of each core substance and period for all PWUO $(n=16,985)$, NSDUH 2002 to 2017

\begin{tabular}{|c|c|c|c|}
\hline \multirow{2}{*}{\multicolumn{2}{|c|}{ Core substances }} & \multicolumn{2}{|c|}{$\begin{array}{l}\text { Rao-Scott } \\
\text { analysis }\end{array}$} \\
\hline & & $\underset{(\mathrm{df})}{\chi^{2}}$ & $\mathrm{p}$-value \\
\hline \multirow[b]{2}{*}{ Binge alcohol } & Binge use in the past month & \multirow{2}{*}{$\begin{array}{c}0.93 \\
(3)\end{array}$} & \multirow[b]{2}{*}{0.82} \\
\hline & $\begin{array}{l}\text { Never or 'no' binge use in } \\
\text { past month }\end{array}$ & & \\
\hline \multirow{2}{*}{ Heavy alcohol } & Heavy use in the past month & \multirow{2}{*}{$\begin{array}{c}38.31 \\
(3)\end{array}$} & \multirow{2}{*}{$<0.0001$} \\
\hline & $\begin{array}{l}\text { Never or 'no' heavy use in } \\
\text { past month }\end{array}$ & & \\
\hline \multirow{2}{*}{$\begin{array}{l}\text { Tranquilizers/ } \\
\text { sedatives }\end{array}$} & Used in the past month & \multirow{2}{*}{$\begin{array}{l}3.10 \\
(3)\end{array}$} & \multirow{2}{*}{0.38} \\
\hline & Not used in the past month & & \\
\hline \multirow{2}{*}{ Cigarettes } & Used in the past month & \multirow{2}{*}{$\begin{array}{c}15.54 \\
(3)\end{array}$} & \multirow{2}{*}{0.001} \\
\hline & Not used in the past month & & \\
\hline \multirow{2}{*}{$\begin{array}{l}\text { Other tobacco } \\
\text { products }\end{array}$} & Used in the past month & \multirow{2}{*}{$\begin{array}{c}14.35 \\
(3)\end{array}$} & \multirow{2}{*}{0.003} \\
\hline & Not used in the past month & & \\
\hline \multirow{2}{*}{ Marijuana } & Used in the past month & \multirow{2}{*}{$\begin{array}{l}2.25 \\
(3)\end{array}$} & \multirow{2}{*}{0.52} \\
\hline & Not used in the past month & & \\
\hline \multirow{2}{*}{ Cocaine } & Used in the past month & \multirow{2}{*}{$\begin{array}{l}3.74 \\
(3)\end{array}$} & \multirow{2}{*}{0.29} \\
\hline & Not used in the past month & & \\
\hline \multirow{2}{*}{ Crack } & Used in the past month & \multirow{2}{*}{$\begin{array}{c}0.43 \\
(3)\end{array}$} & \multirow{2}{*}{0.93} \\
\hline & Not used in the past month & & \\
\hline \multirow{2}{*}{$\begin{array}{c}\text { Hallucinogens/ } \\
\text { inhalants }\end{array}$} & Used in the past month & \multirow{2}{*}{$\begin{array}{c}8.14 \\
(3)\end{array}$} & \multirow{2}{*}{0.043} \\
\hline & Not used in the past month & & \\
\hline \multirow{2}{*}{ Stimulants } & Used in the past month & \multirow{2}{*}{$\begin{array}{c}11.31 \\
(3)\end{array}$} & \multirow{2}{*}{0.01} \\
\hline & Not used in the past month & & \\
\hline
\end{tabular}

Abbreviations: $\chi^{2}(\mathrm{df})=$ Chi-square statistic (degrees of freedom)

Note: Bolded $\chi^{2}$ and $p$-values denote statistical significance at $\mathrm{p}<0.05$.

Joinpoint regression was used again to examine trends in the substances that were significantly associated with period. The results for all five of these substances were shown in Figures 4.12 through 4.16. (For full joinpoint regression results, see ST 4.7a-e in Appendix A). Although not significant, the trend in the prevalence of heavy alcohol drinking in PWUO increased from 2002 to 2006; however, from 2006 to 2017, the 
Annual Percent Change (APC) indicated a significant negative trajectory of reported heavy alcohol drinking [APC (p-value): $-2.70(<0.001)]$. For past month cigarette use among PWUO, no significant trend was found using joinpoint regression [APC (p-value): $0.11(0.50)]$. Also, no significant trends were found for the prevalence of other tobacco product use [APC (p-value): $0.20(0.50)]$ and hallucinogen/inhalant use [APC (p-value): $0.10(0.80)]$. However, joinpoint regression modeled three time-segments in the prevalence trend of stimulant use in PWUO. Although the trends for the time-segments 1 and 2 were not significant, the trend from 2007 to 2017 indicated a significant increase in the APC for the prevalence of past month stimulant use in PWUO [APC (p-value): 5.4 (< 0.001)]

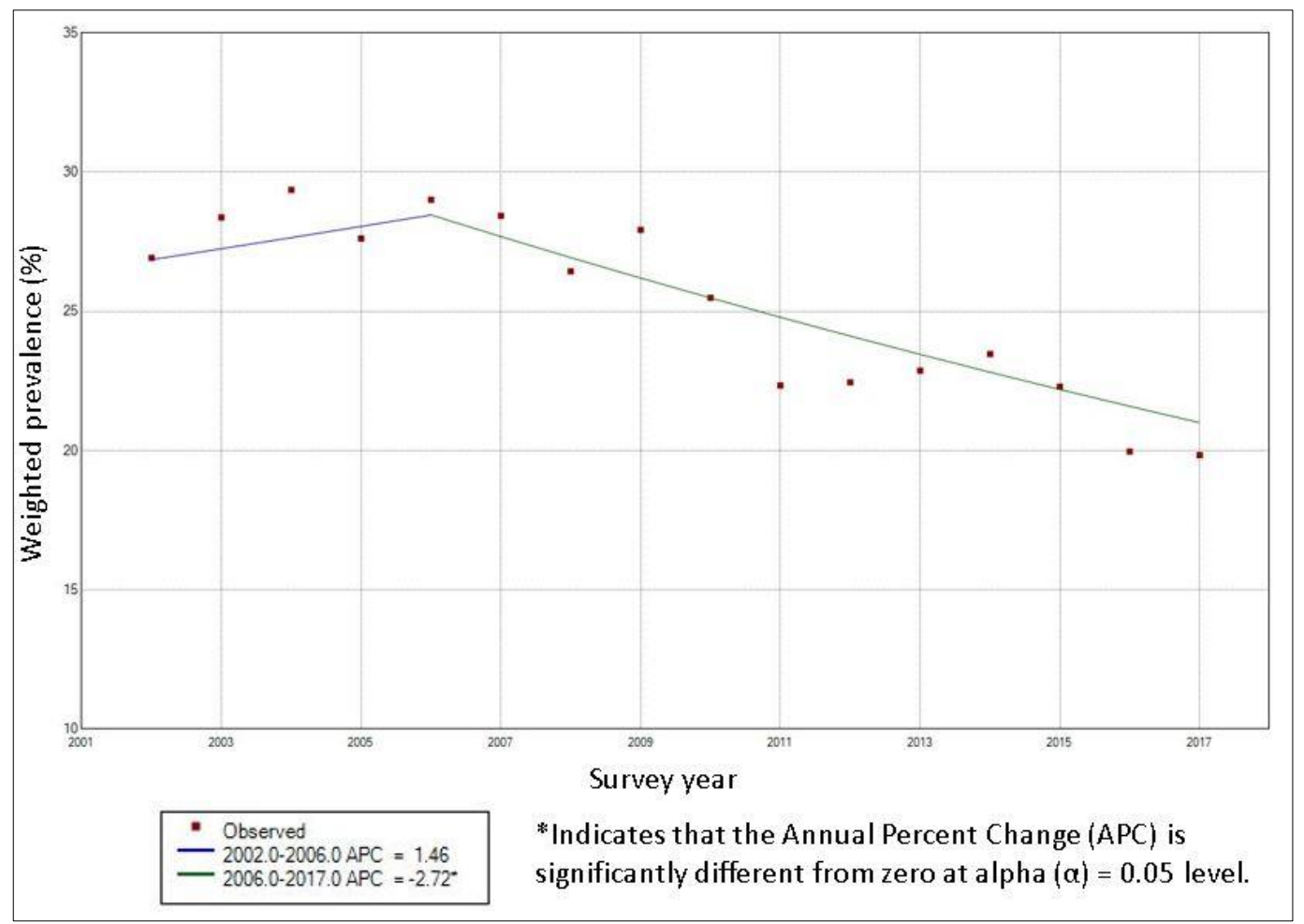

Figure 4.12 $\mid$ Joinpoint analysis of the weighted prevalence of past month heavy alcohol use among all PWUO $(\mathrm{n}=16,985)$ 


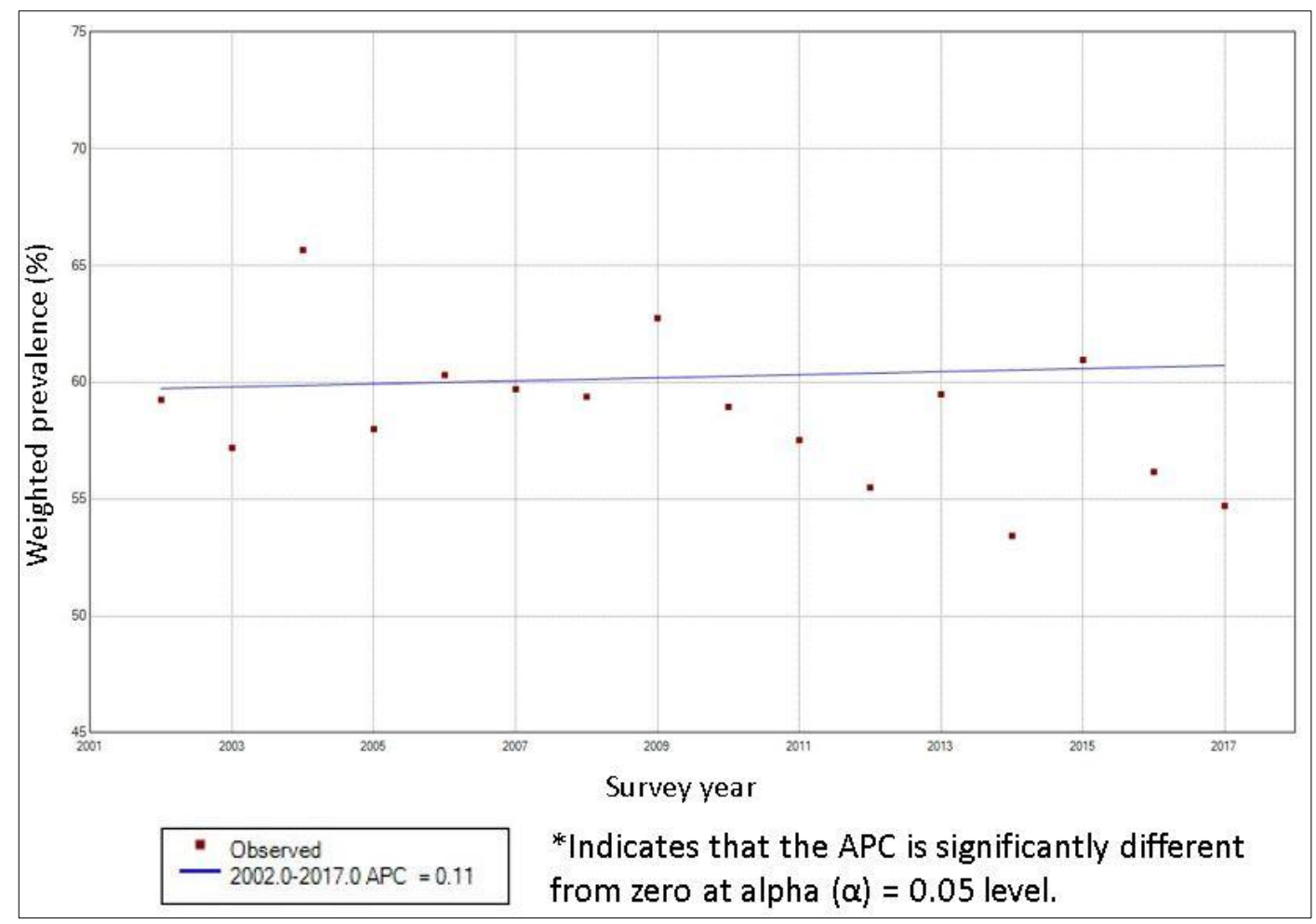

Figure 4.13 $\mid$ Joinpoint analysis of the weighted prevalence of past month cigarette use among all PWUO $(\mathrm{n}=16,985)$

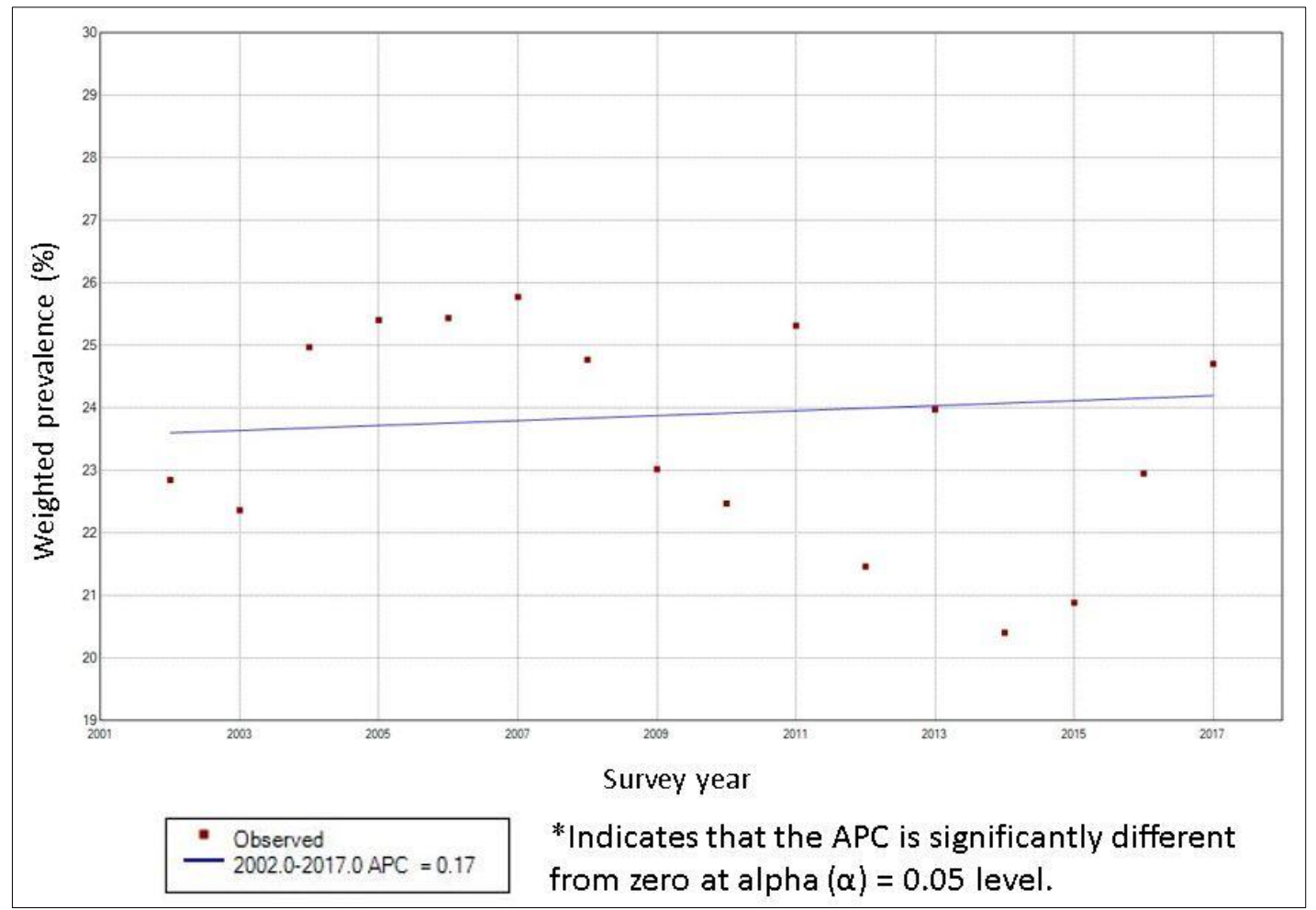

Figure 4.14 $\mid$ Joinpoint analysis of the weighted prevalence of past month other tobacco products use among all PWUO $(\mathrm{n}=16,985)$ 


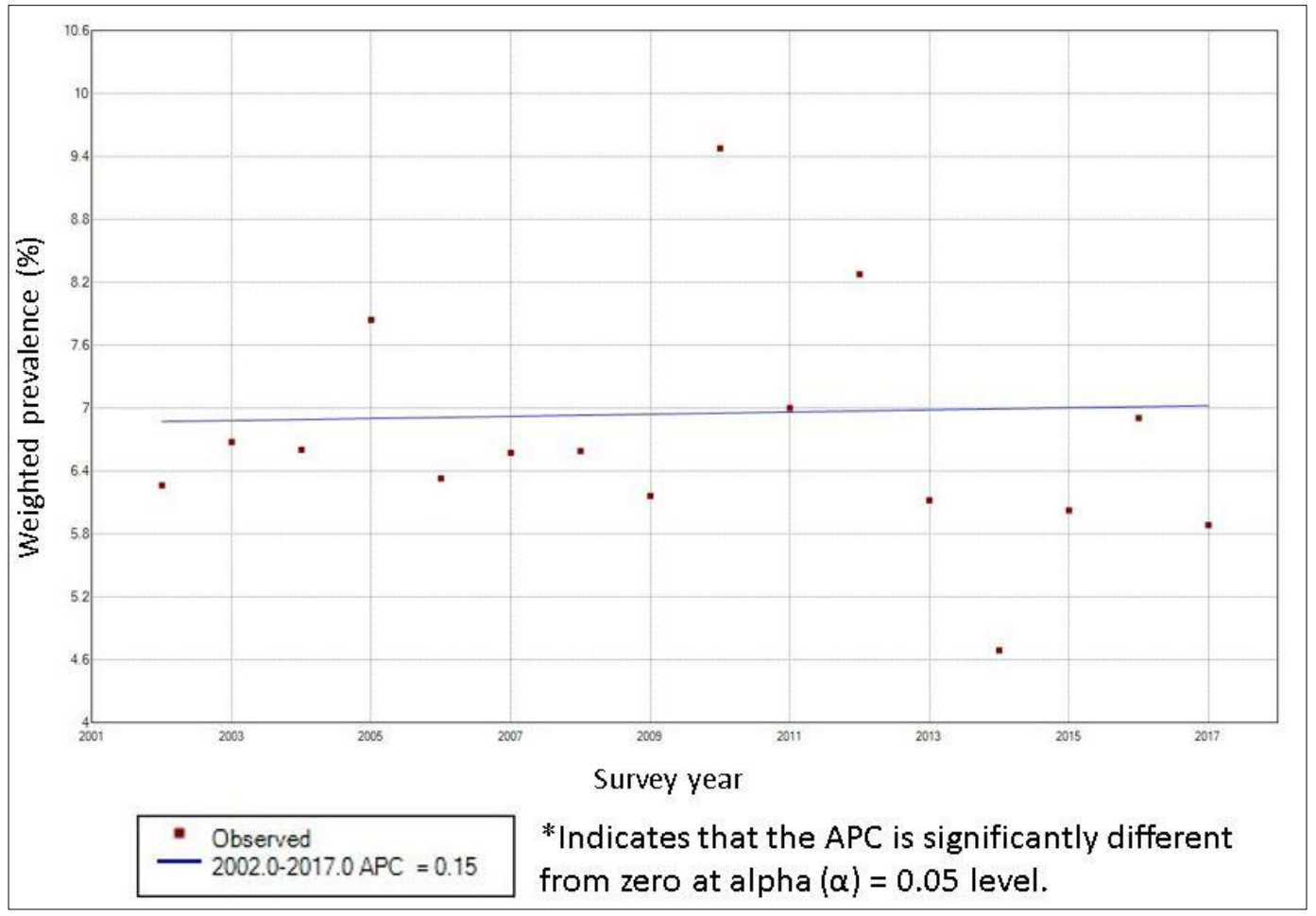

Figure 4.15 $\mid$ Joinpoint analysis of the weighted prevalence of past month hallucinogen/inhalant use among all PWUO $(n=16,985)$

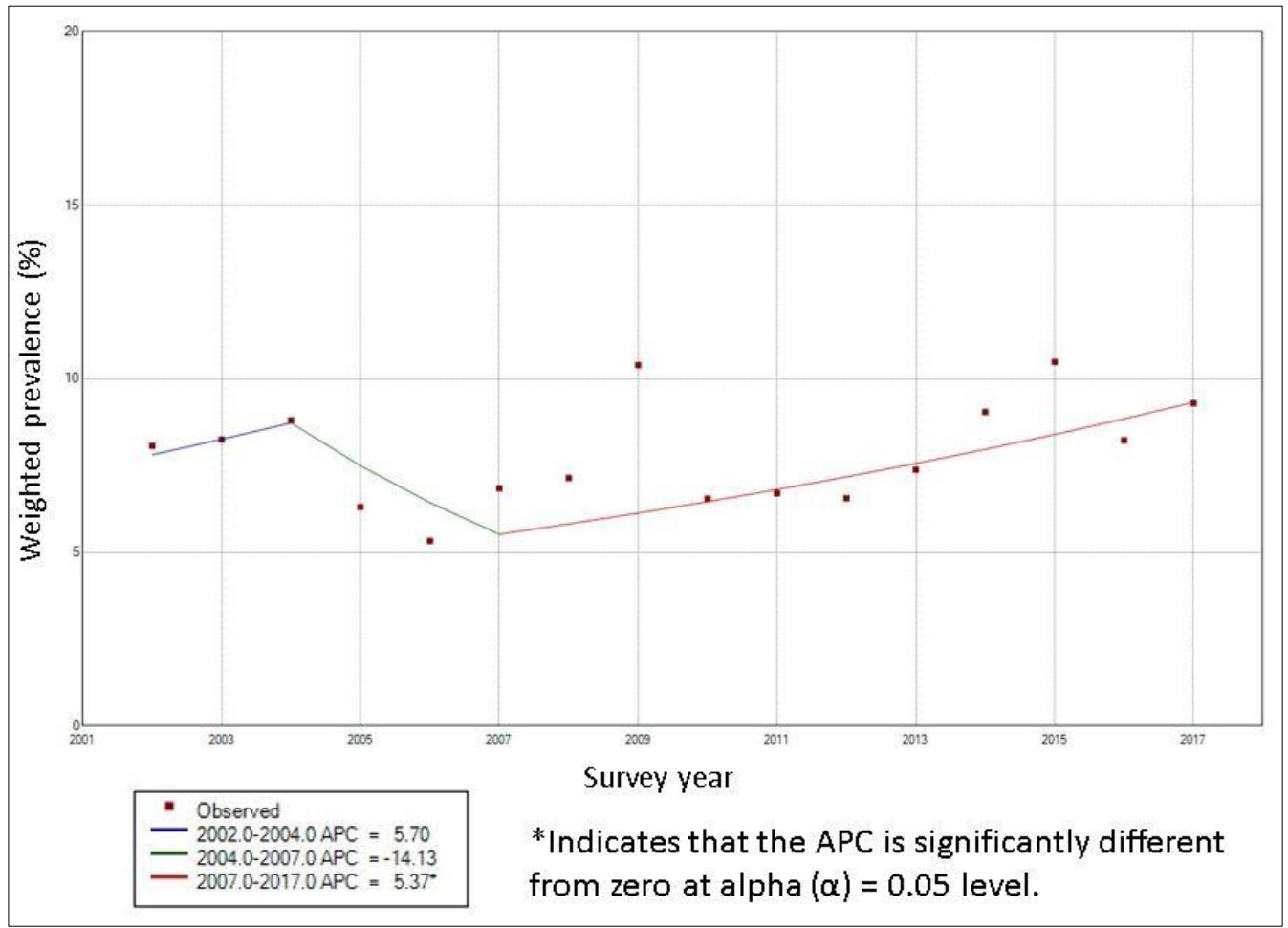

Figure 4.16 | Joinpoint analysis of the weighted prevalence of past month stimulant use among all PWUO $(n=16,985)$ 


\section{Prevalence of substance use among opioid use groups}

Past month PPR-only use group

Figure 4.17 shows the overall weighted prevalence for the past month reported use of all core substances found in the NSDUH among the past month PPR-only group. The substances that were reported by the past month PPR-only use group from largest to smallest prevalence were cigarettes, marijuana, binge alcohol drinking, heavy alcohol drinking, other tobacco use, tranquilizers and sedatives, cocaine, stimulants, hallucinogens and inhalants, and crack. One distinguishing characteristic of this group was the low prevalence of reported misuse of prescription drugs (stimulants, tranquilizers/ sedatives) and illicit substances (cocaine, hallucinogens/inhalants, crack). (For all overall prevalence results for this use group, see ST 4.8 in Appendix A). period

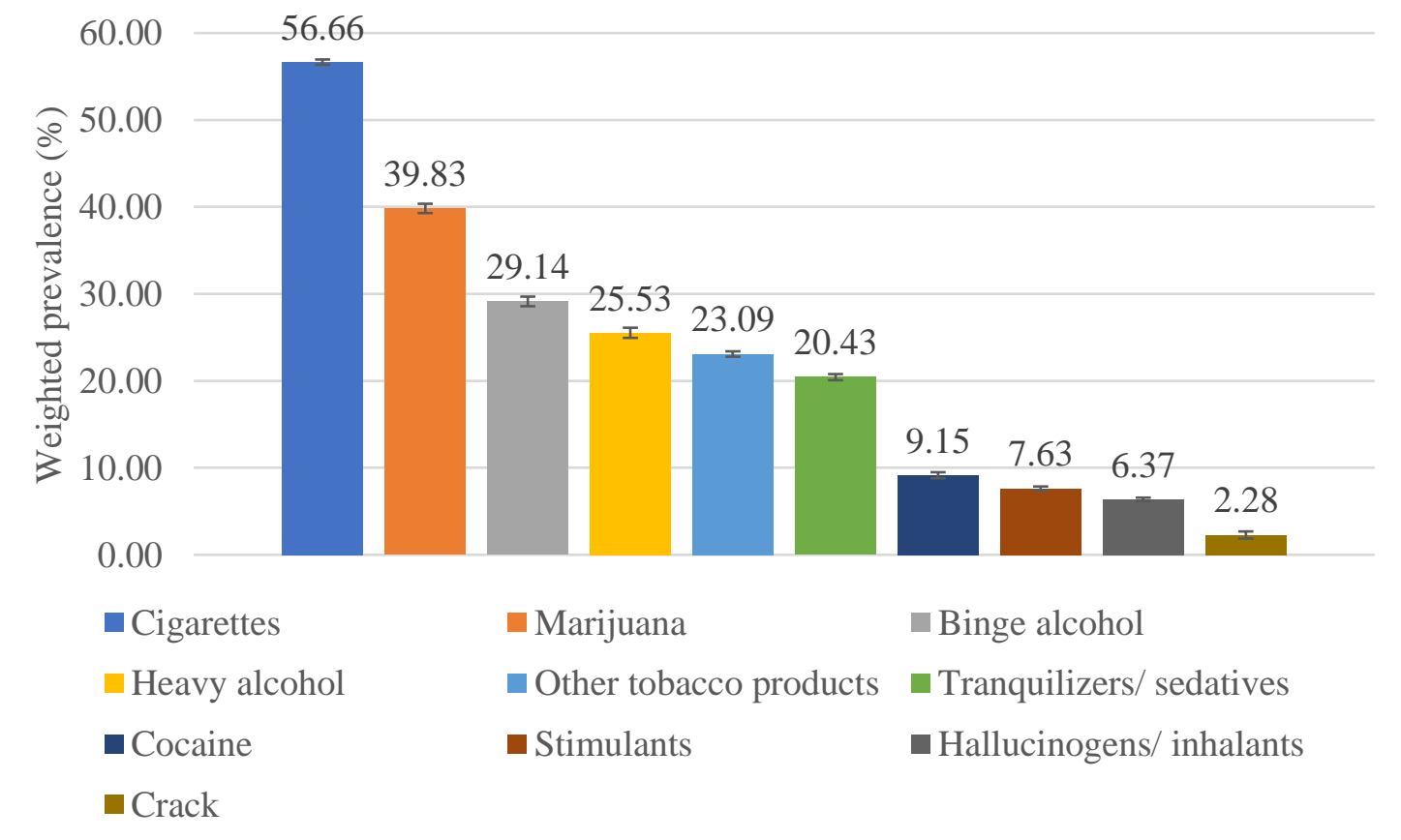

Figure 4.17 | Overall weighted prevalence of past month use of all core substances among the past month PPR-only group ( $\mathrm{n}=15,897)$, NSDUH 2002 to 2017 


\section{Past month heroin-only use group}

As shown in Figure 4.18, the prevalence of past month cigarette use was highest in this group, which was followed by the prevalence of past month marijuana, cocaine, binge alcohol, other tobacco products, crack, heavy alcohol, tranquilizer/sedative, hallucinogen/inhalant, and stimulant use. One distinguishing characteristic of this group was the increase in the prevalence of reported past month use of cocaine and crack use, especially when compared to the past month PPR-only use group. (For all overall prevalence results among this use group, see ST 4.9 in Appendix A).

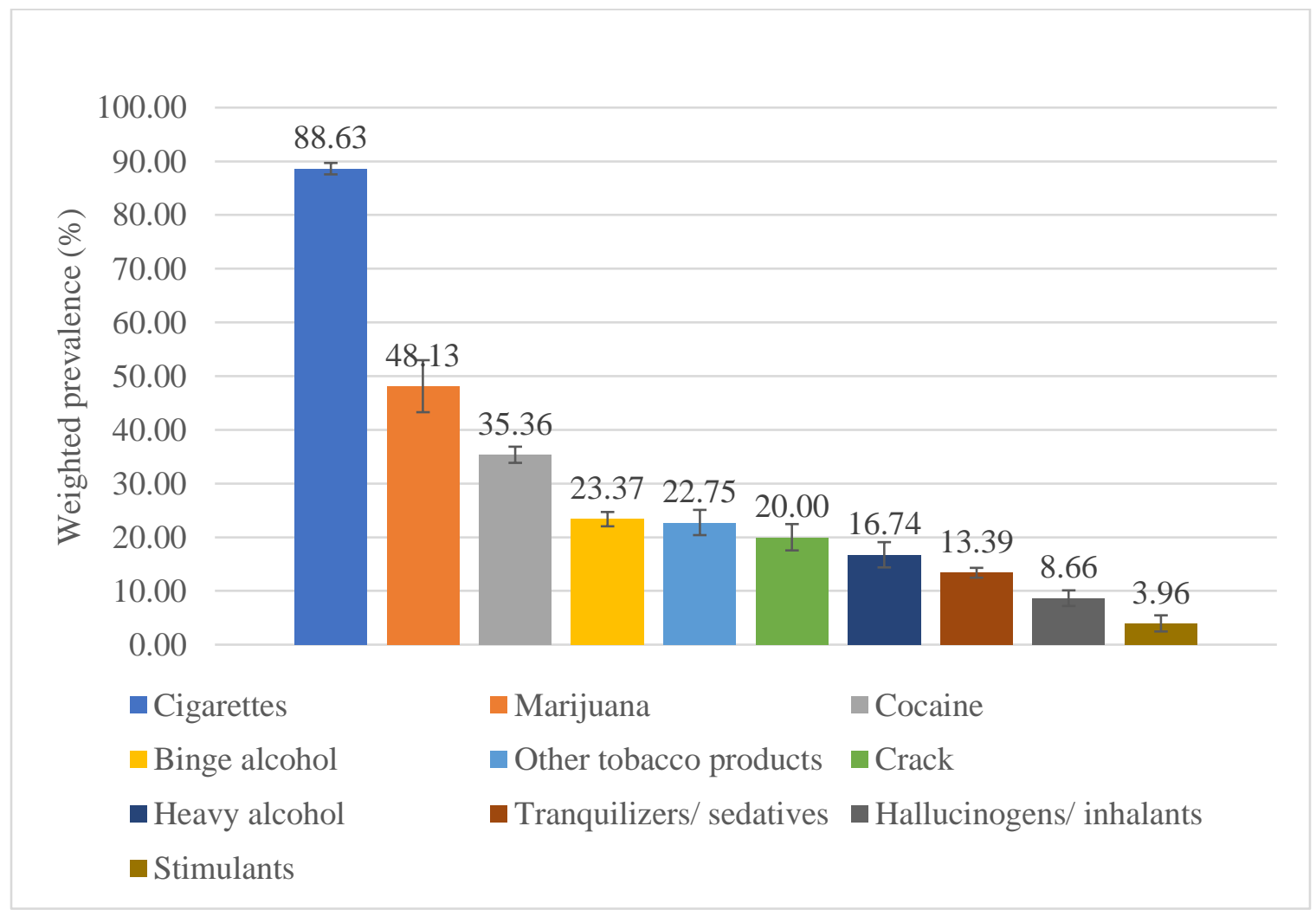

Figure 4.18 | Overall weighted prevalence of past month use of all core substances among the past month heroin-only group $(\mathrm{n}=553)$, NSDUH 2002 to 2017 


\section{Past month combination use group}

As shown in Figure 4.19, cigarettes continued to make up the highest proportion of reported substance use, followed by the prevalence of reported past month marijuana, cocaine, tranquilizer/sedative, other tobacco products, heavy alcohol, crack, binge alcohol, stimulant, and hallucinogen/inhalant use. Also, the prevalence of past month cocaine and tranquilizer/sedative use in this group exceeded that of the past month heroin-only use group. On average, the prevalence of heavy and binge alcohol use ranged from $25 \%$ to $30 \%$ which was comparable to the past month PPR-only use group and was greater than among members of the heroin-only use group. (For all overall prevalence results in this use group, see ST 4.10 in Appendix A).

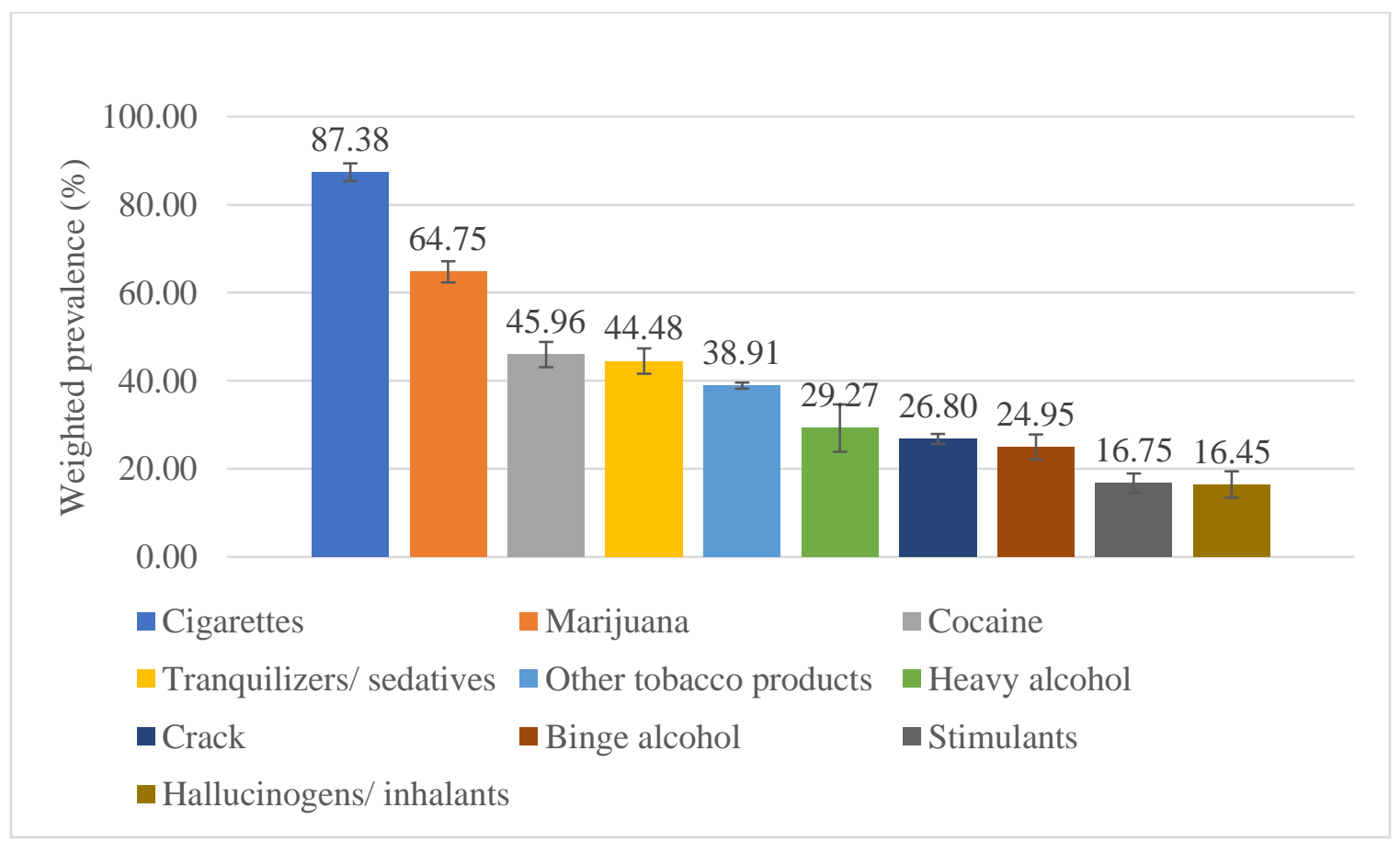

Figure 4.19 Overall weighted prevalence of past month use of all core substances among the past month combination opioid group $(n=535)$, NSDUH 2002 to 2017 


\section{Longitudinal trends of substance use among opioid use groups}

\section{Past month PPR-only use group}

From a qualitative assessment of Figures 4.20, 4.21, and 4.22, the trajectory of the prevalence of the reported use of all substances generally shows an increase from period 1 to 2 . However, prevalence declined for most substances after period 2. Also, there were only a few substances whose prevalence were significantly associated with period; the bivariable $\chi^{2}$ analysis indicated significant associations between period and the weighted prevalence of past month heavy alcohol drinking $\left[\chi^{2}(\mathrm{df}),(\mathrm{p}\right.$-value): 27.26 (3), $(<0.0001)]$, past month cigarette use $\left[\chi^{2}(\mathrm{df}),(\mathrm{p}\right.$-value): $57.54(3),(<0.0001)]$, past month other tobacco products use [ $\chi^{2}(\mathrm{df}),(\mathrm{p}$-value): $20.87(3),(0.0001)]$, and past month hallucinogen/inhalant use [ $\chi^{2}(\mathrm{df})$, (p-value): $\left.8.12(3),(0.044)\right]$, as shown in Table 4.4. (For detailed prevalence results by period for this use group, see ST 4.11 in Appendix A).

Joinpoint regression was again used to determine whether a significant trend existed for the prevalence of cigarette, other tobacco products, and hallucinogen/inhalant use for this group, and the results are shown in Figures 4.23 through 4.26. Trend analysis of the prevalence of past month heavy alcohol drinking revealed a significant decrease from 2007 to 2017 [APC (p-value): -2.70 (<0.001)]. Although no joinpoints were found in any of the trajectories for the other three substances, the results indicated that the prevalence trend of cigarettes [APC (p-value): $-0.90(<0.001)]$ and hallucinogens/inhalants [APC (p-value): -1.10 (0.10)] were decreasing from 2002 to 2017. However, the prevalence of past month use of other tobacco products significantly 
increased from 2002 to 2017 [APC (p-value): $0.40(<0.001)$ ]. (For detailed joinpoint regression results for this use group, see ST 4.12a-c in Appendix A).

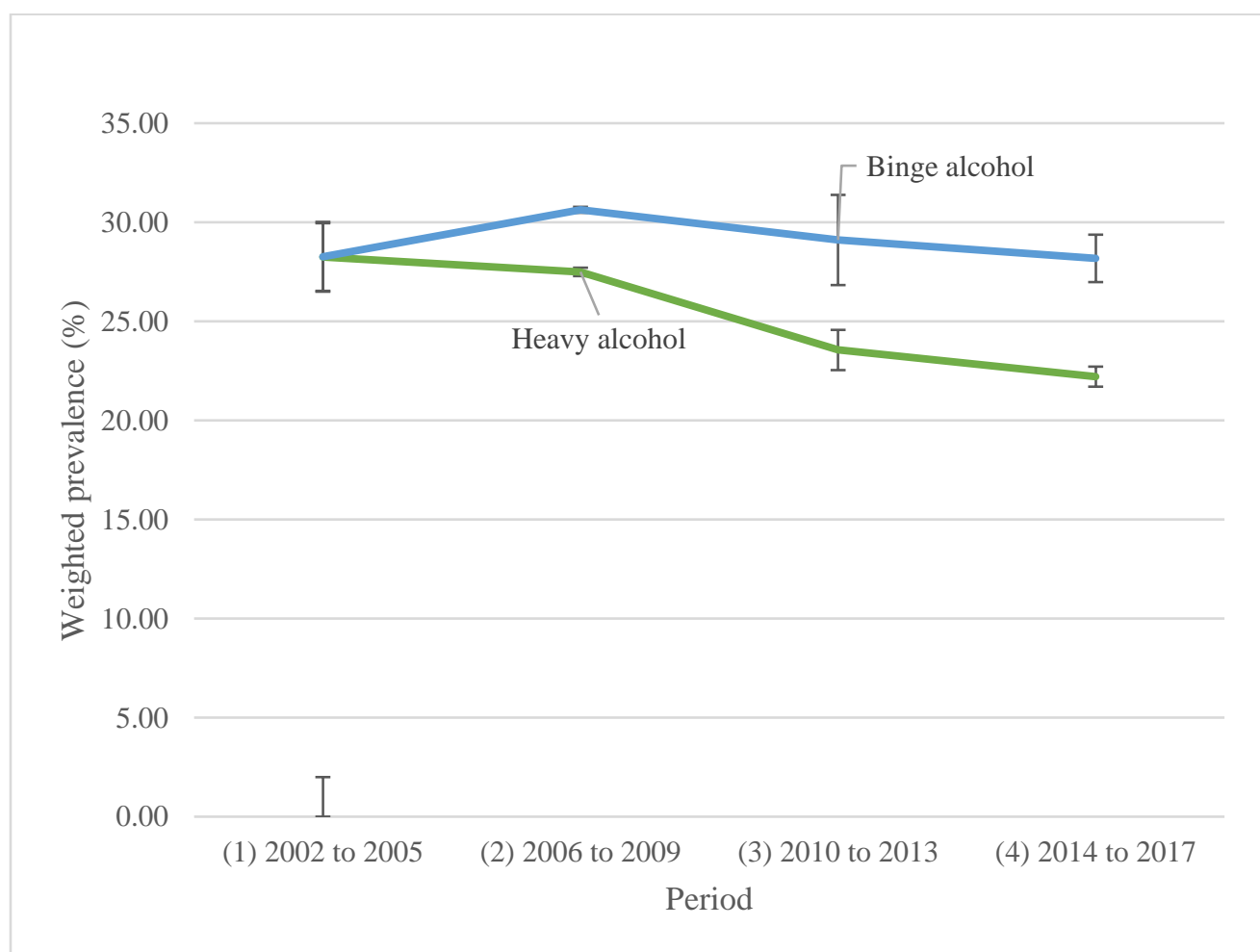

Figure 4.20 | Weighted prevalence of all levels of past month alcohol drinking among past month PPR-only group by period $(\mathrm{n}=15,897)$, NSDUH 2002 to 2017 


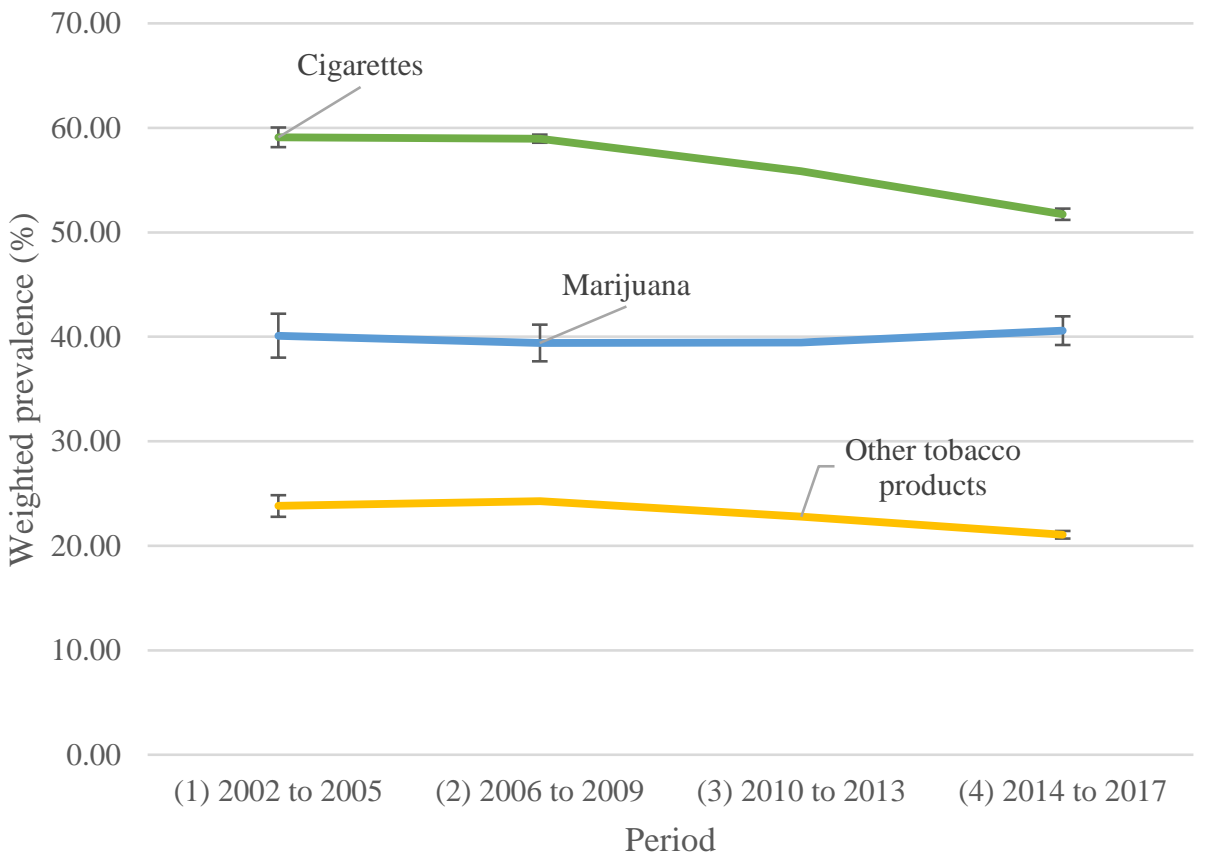

Figure 4.21 | Weighted prevalence of reported past month cigarette smoking, other tobacco use, and marijuana use among past month PPR-only use group by period $(n=15,897)$, NSDUH 2002 to 2017

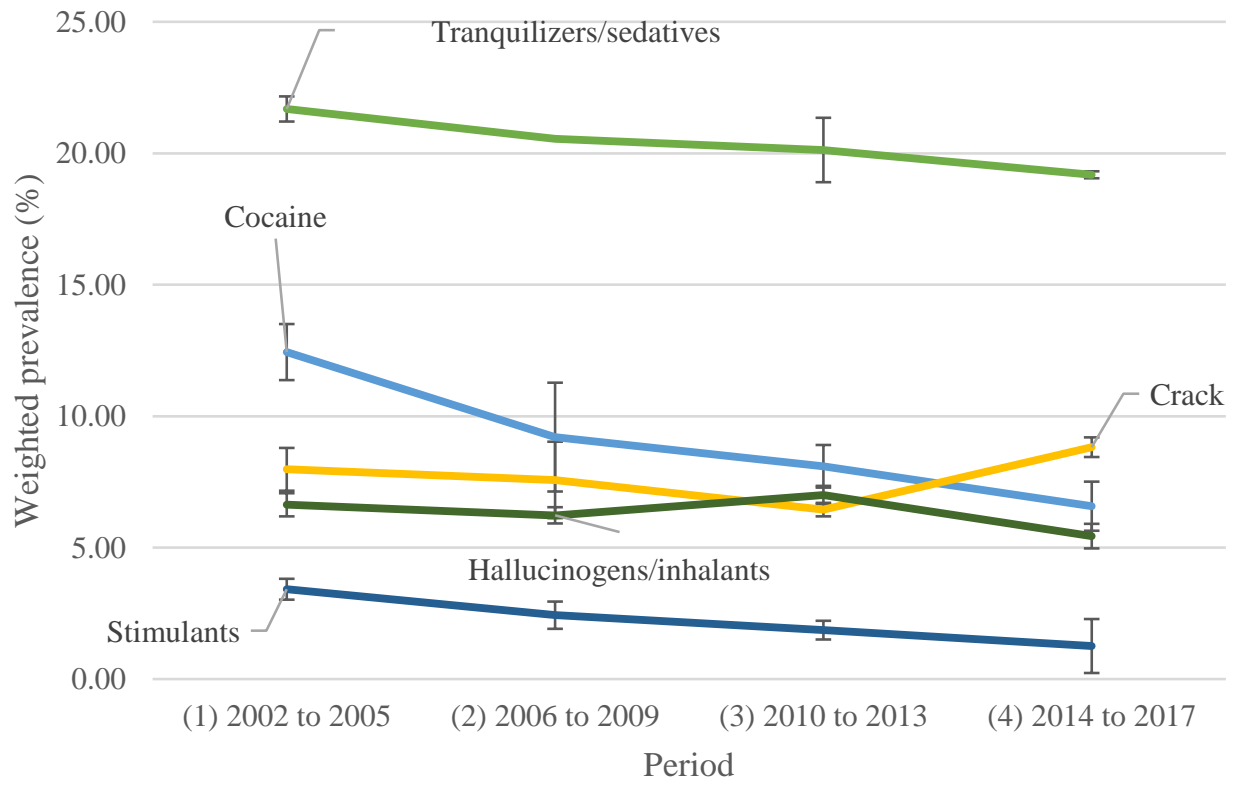

Figure 4.22 | Weighted prevalence of reported past month tranquilizer/sedative, cocaine, crack, hallucinogen/inhalant, and stimulant use among past month PPR-only use group by period $(\mathrm{n}=15,897)$, NSDUH 2002 to 2017 
Table 4.4 $\mid$ Rao-Scott $\chi^{2}$ analyses for the association of prevalence of each core substance and period for past month PPR-only use group $(n=15,897)$, NSDUH 2002 to 2017

\begin{tabular}{|c|c|c|c|}
\hline \multirow{2}{*}{\multicolumn{2}{|c|}{ Core substances }} & \multicolumn{2}{|c|}{ Rao-Scott analysis } \\
\hline & & $\chi^{2}(\mathrm{df})$ & $\mathrm{p}$-value \\
\hline \multirow{2}{*}{ Binge alcohol } & Binge use in the past month & \multirow{2}{*}{$\begin{array}{l}1.40 \\
(3)\end{array}$} & \multirow{2}{*}{0.7056} \\
\hline & $\begin{array}{c}\text { Never or 'no' binge use in } \\
\text { past month }\end{array}$ & & \\
\hline \multirow{2}{*}{ Heavy alcohol } & Heavy use in the past month & \multirow{2}{*}{$\begin{array}{c}27.26 \\
(3)\end{array}$} & \multirow{2}{*}{$<0.0001$} \\
\hline & $\begin{array}{l}\text { Never or 'no' heavy use in } \\
\text { past month }\end{array}$ & & \\
\hline \multirow{2}{*}{$\begin{array}{l}\text { Tranquilizers/ } \\
\text { sedatives }\end{array}$} & Used in the past month & \multirow{2}{*}{$\begin{array}{c}6.52 \\
(3)\end{array}$} & \multirow{2}{*}{0.089} \\
\hline & Not used in the past month & & \\
\hline \multirow{2}{*}{ Cigarettes } & Used in the past month & \multirow{2}{*}{$\begin{array}{c}57.54 \\
(3)\end{array}$} & \multirow{2}{*}{$<0.0001$} \\
\hline & Not used in the past month & & \\
\hline \multirow{2}{*}{$\begin{array}{l}\text { Other tobacco } \\
\text { products }\end{array}$} & Used in the past month & \multirow{2}{*}{$\begin{array}{c}20.87 \\
(3)\end{array}$} & \multirow{2}{*}{0.0001} \\
\hline & Not used in the past month & & \\
\hline \multirow{2}{*}{ Marijuana } & Used in the past month & \multirow{2}{*}{$\begin{array}{c}0.32 \\
(3)\end{array}$} & \multirow{2}{*}{0.96} \\
\hline & Not used in the past month & & \\
\hline \multirow{2}{*}{ Cocaine } & Used in the past month & \multirow{2}{*}{$\begin{array}{l}7.46 \\
(3)\end{array}$} & \multirow{2}{*}{0.059} \\
\hline & Not used in the past month & & \\
\hline \multirow{2}{*}{ Crack } & Used in the past month & \multirow{2}{*}{$\begin{array}{l}3.07 \\
(3)\end{array}$} & \multirow{2}{*}{0.38} \\
\hline & Not used in the past month & & \\
\hline \multirow{2}{*}{$\begin{array}{l}\text { Hallucinogens/ } \\
\text { inhalants }\end{array}$} & Used in the past month & \multirow{2}{*}{$\begin{array}{c}8.12 \\
(3)\end{array}$} & \multirow{2}{*}{0.044} \\
\hline & Not used in the past month & & \\
\hline \multirow{2}{*}{ Stimulants } & Used in the past month & \multirow{2}{*}{$\begin{array}{l}7.15 \\
(3)\end{array}$} & \multirow{2}{*}{0.067} \\
\hline & Not used in the past month & & \\
\hline
\end{tabular}

Abbreviations: $\chi^{2}(\mathrm{df})=$ Chi-square statistic (degrees of freedom)

Note: Bolded $\chi^{2}$ and $p$-values denote statistical significance at $\mathrm{p}<0.05$. 


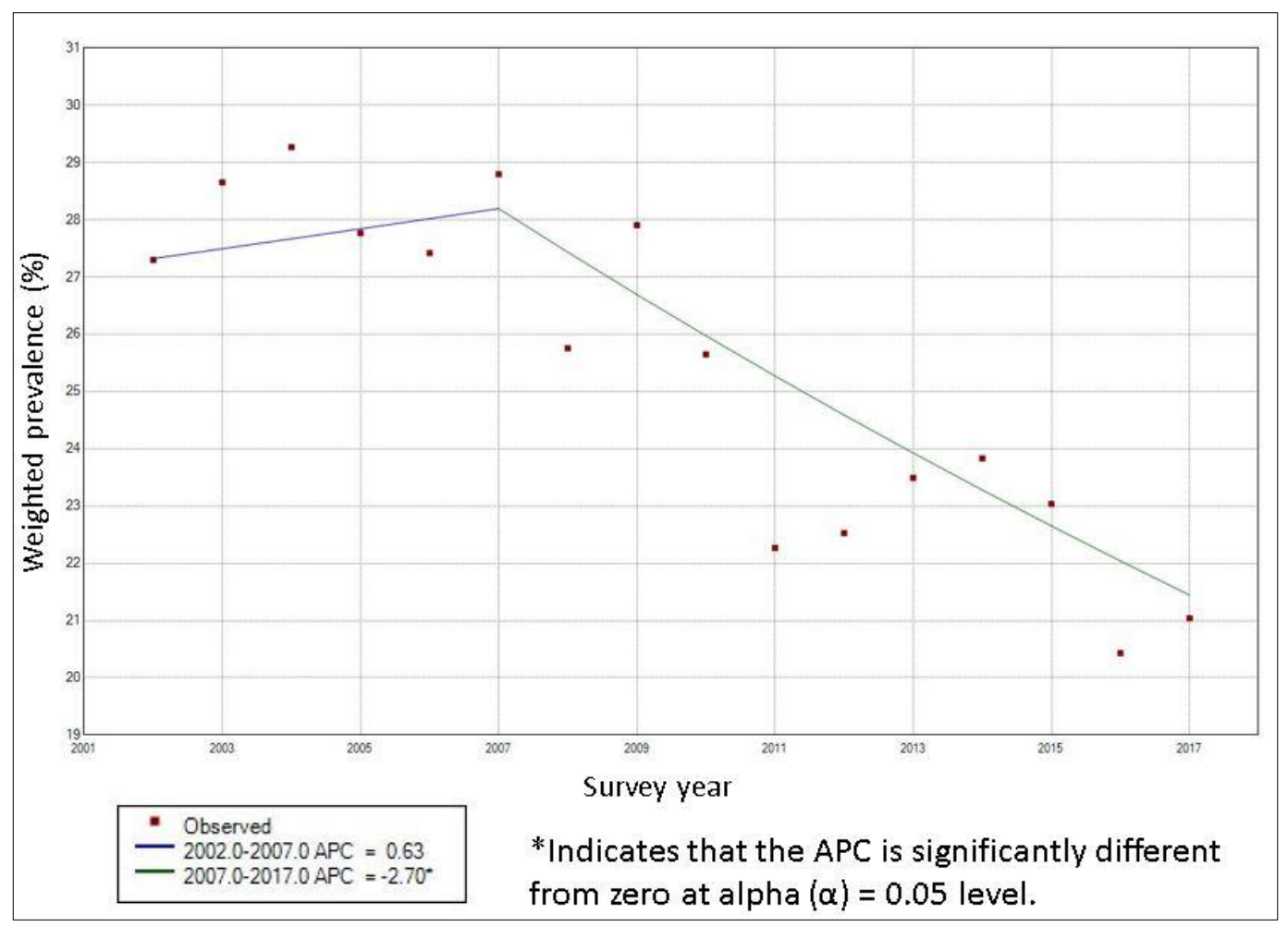

Figure 4.23 $\mid$ Joinpoint analysis of the weighted prevalence of past month heavy alcohol use among past month PPR-only use group $(n=15,897)$

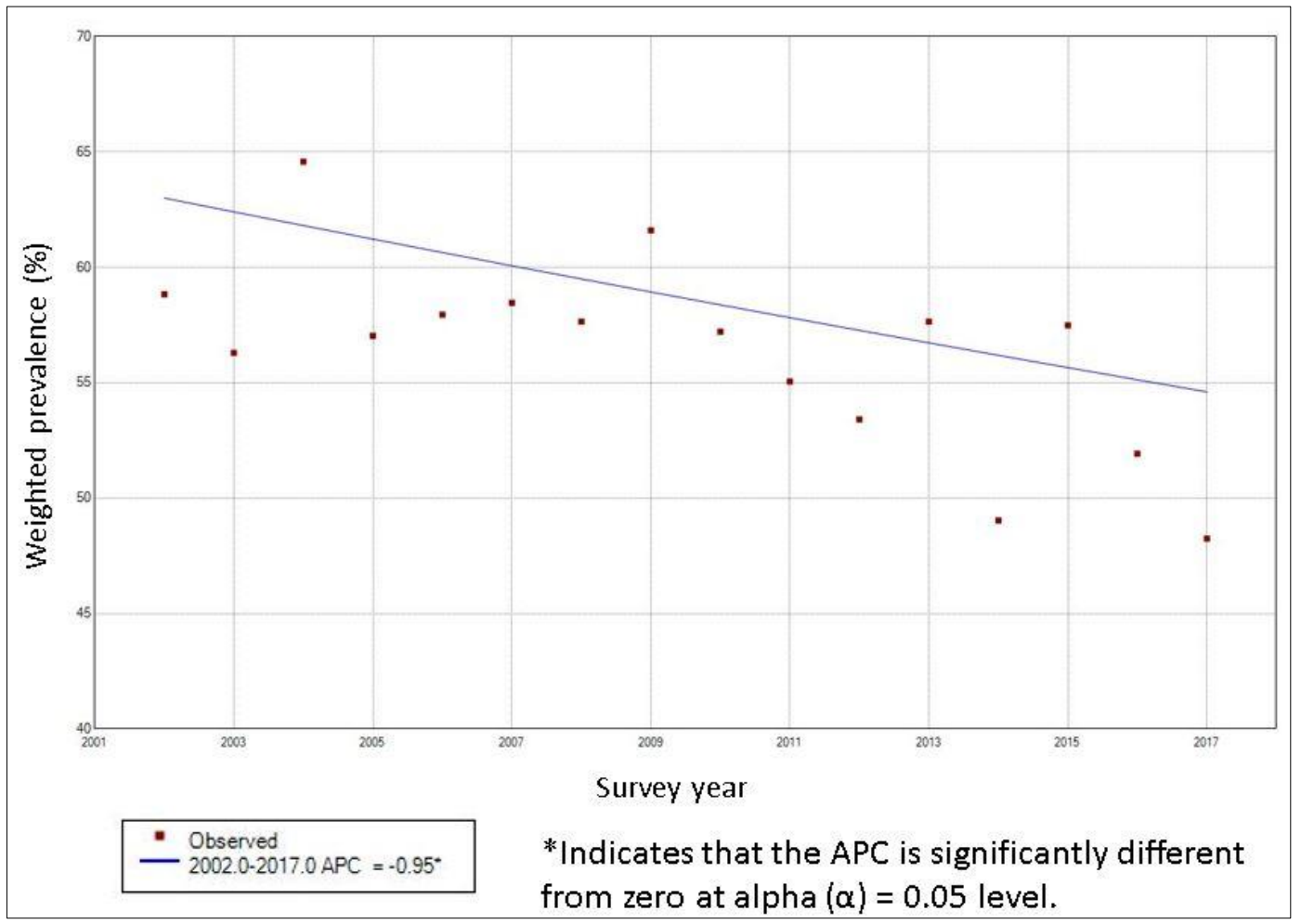

Figure 4.24 | Joinpoint analysis of the weighted prevalence of past month cigarette use among past month PPR-only use group $(\mathrm{n}=15,897)$ 


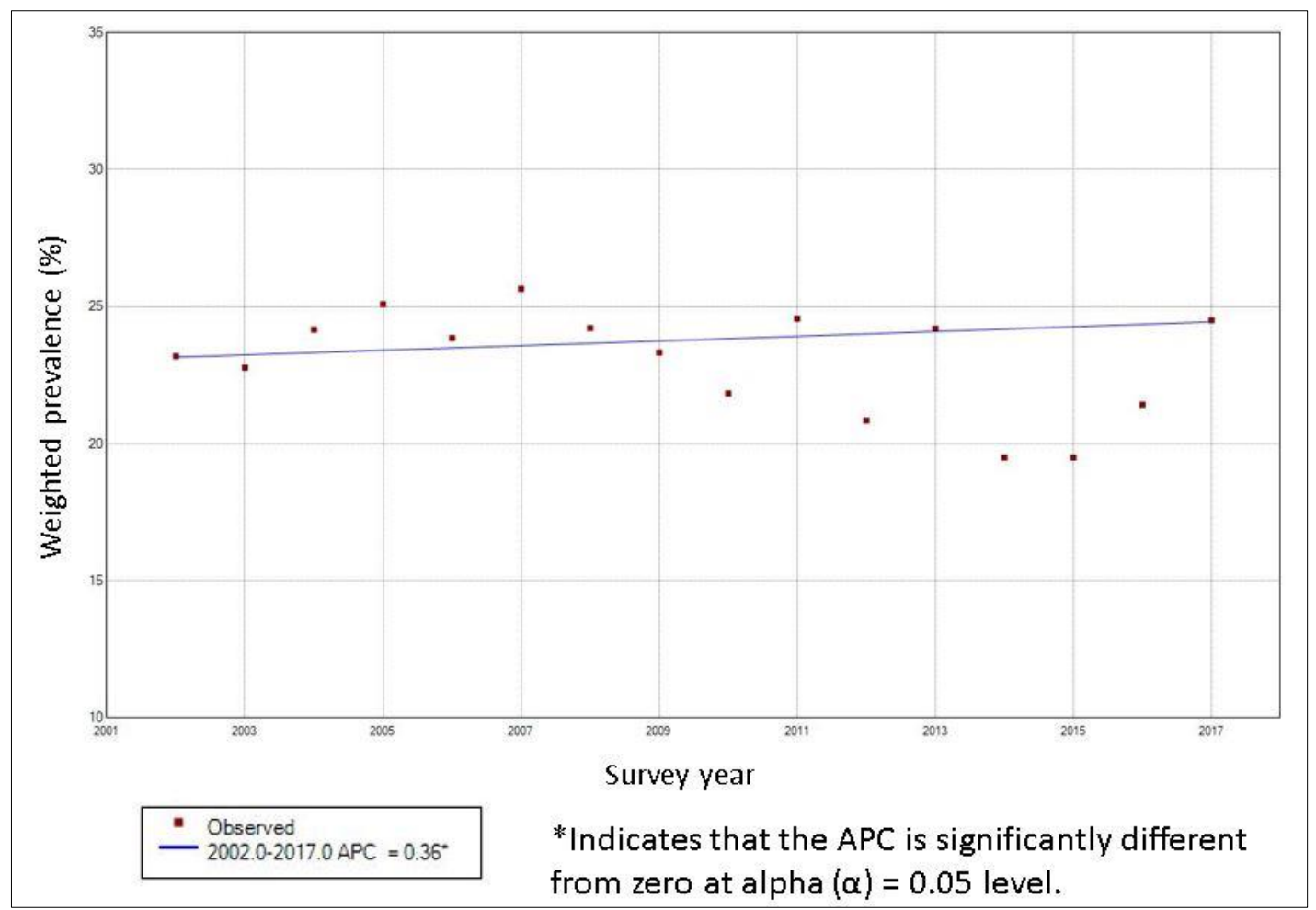

Figure 4.25 $\mid$ Joinpoint analysis of the weighted prevalence of past month other tobacco use among past month PPR-only use group $(n=15,897)$

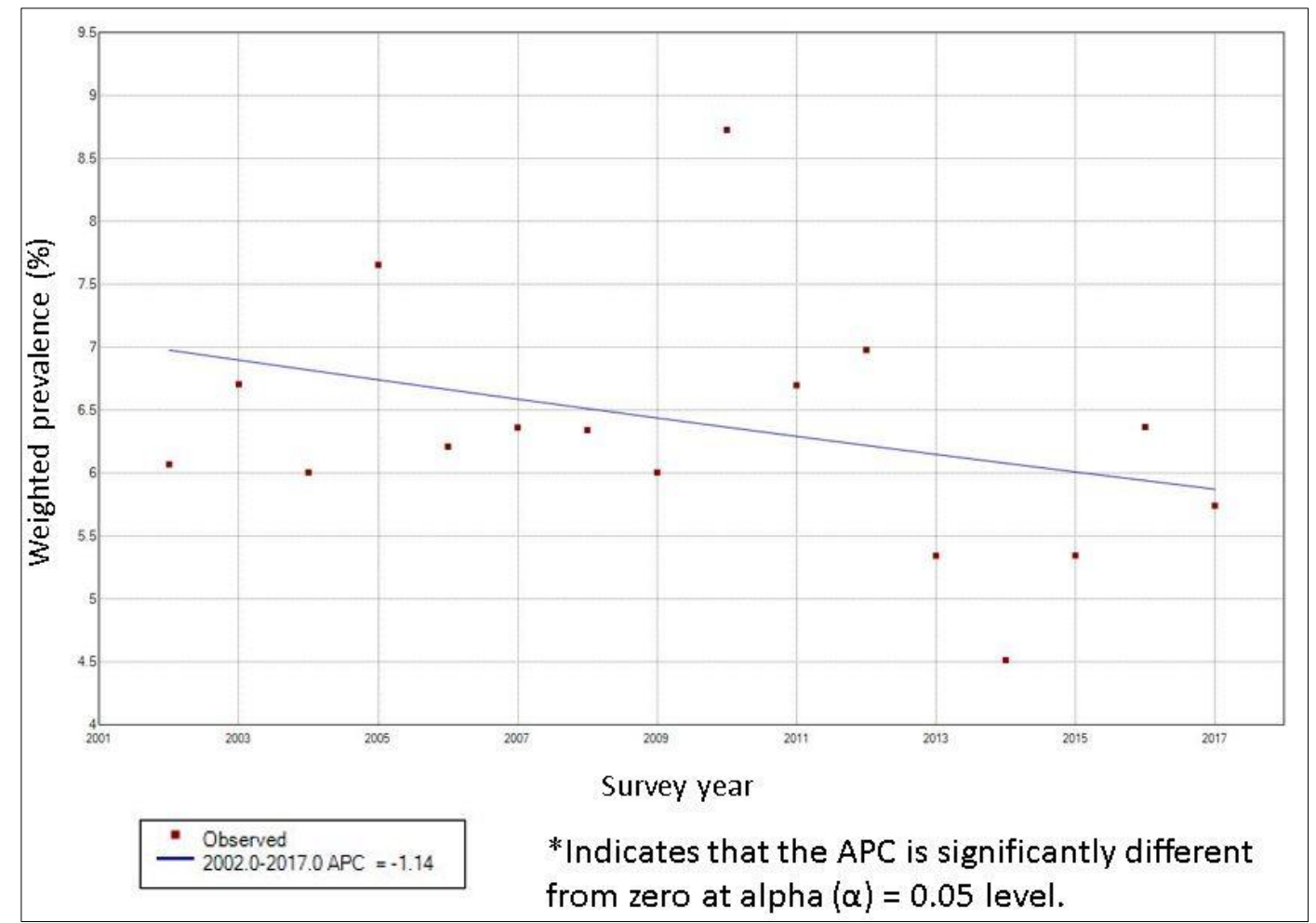

Figure 4.26 | Joinpoint analysis of the weighted prevalence of past month hallucinogen/inhalant use among past month PPR-only use group $(n=15,897)$ 


\section{Past month heroin-only use group}

Initial assessment of the visual trends displayed in Figures 4.27, 4.28, and 4.29 shows that weighted prevalence of reported past month cigarette use continued to dominate the prevalence of all other substances from period 1 to 4 . While patterns of prevalence were heterogeneous, many of the longitudinal differences in weighted prevalence for most substances appeared to be decreasing or remaining somewhat constant (e.g., other tobacco products, stimulants, crack, cocaine). Also, the prevalence of past month cigarette use decreased from $95.34 \%(\mathrm{SE}=2.52$ ) in period 1 to $85.75 \%$ $(\mathrm{SE}=1.81)$ in period 2 , with a gradual increase from period 3 to 4 . Additionally, the weighted prevalence of past month use of marijuana and binge alcohol drinking showed a consistent increase after period 2. (For all prevalence results by period, see ST 4.13 in Appendix A for full results)

The results of the $\chi^{2}$ analysis between period and prevalence of reported past month substance use are shown in Table 4.5. They indicated that the weighted prevalence of two substances had a significant association with period: heavy alcohol use $\left[\chi^{2}(\mathrm{df}),(\mathrm{p}\right.$-value): $8.13(3),(0.0434)]$ and cigarettes [ $\chi^{2}(\mathrm{df}),(\mathrm{p}$-value): $8.26(3)$, (0.041)]. Although no joinpoints were detected, a non-significant negative trend in the prevalence of past month heavy alcohol [m (p-value): -0.66 (0.10)] was detected as shown in Figure 4.30. From 2002 to 2008, cigarette use among this group increased, but subsequently significantly decreased from 2008 to 2017 [APC (p-value): $-2.3(<0.001)$ ] as shown in Figure 4.31. (For full joinpoint regression results for this opioid use group, see ST 4.14a-b in Appendix A). 


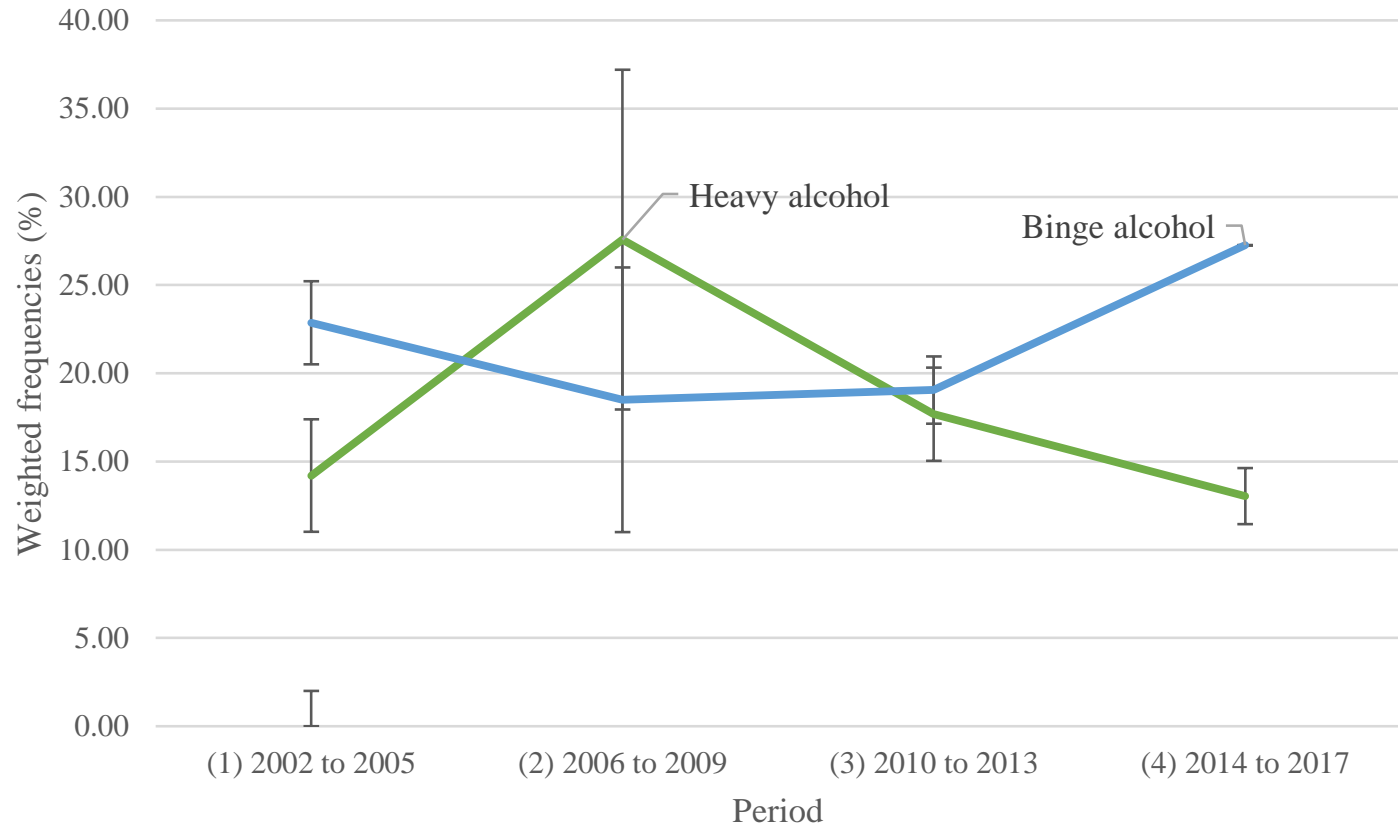

Figure 4.27 | Weighted prevalence of past month binge and heavy alcohol drinking among past month heroin-only use group by period $(n=553)$, NSDUH 2002 to 2017

120.00

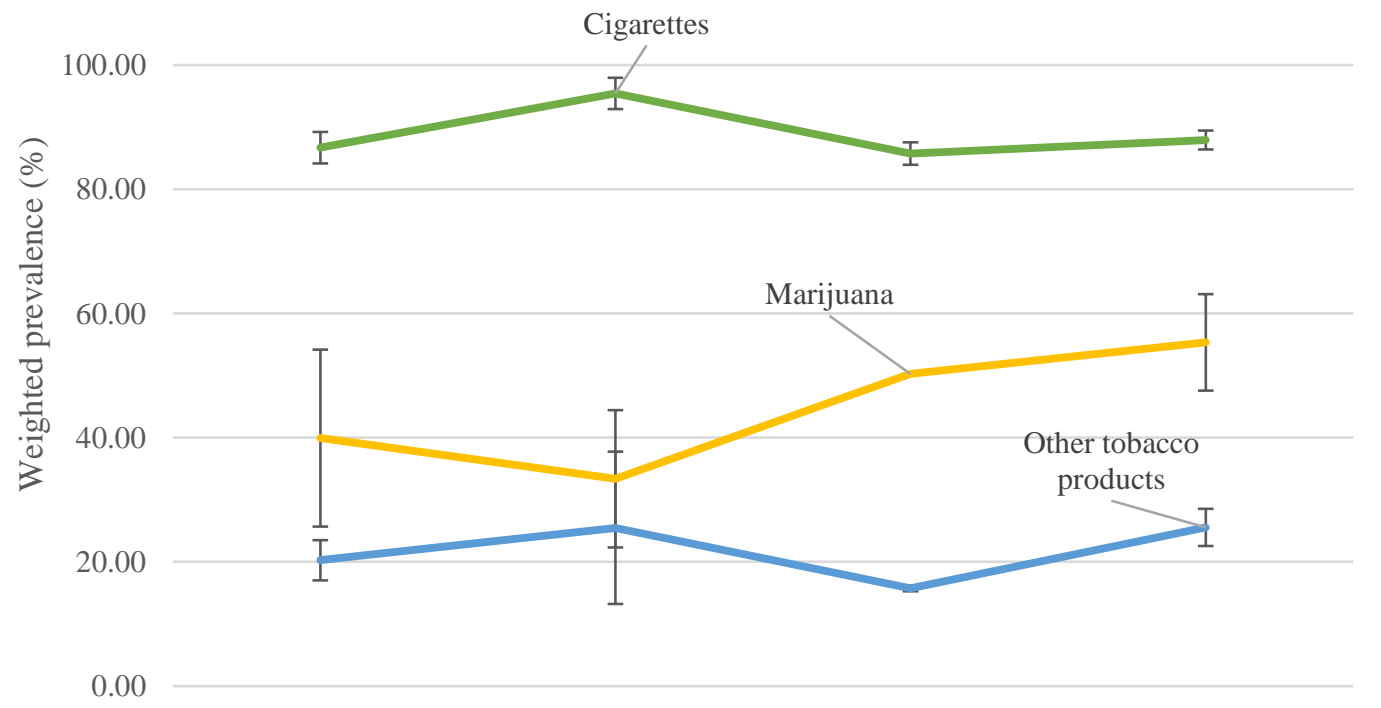

$\begin{array}{llll}\text { (1) } 2002 \text { to } 2005 & \text { (2) } 2006 \text { to } 2009 & \text { (3) } 2010 \text { to } 2013 & \text { (4) } 2014 \text { to } 2017\end{array}$

Period

Figure 4.28 | Weighted prevalence of reported past month cigarette smoking, other tobacco use, and marijuana use among past month heroin-only use group by period $(n=553)$, NSDUH 2002 to 2017 


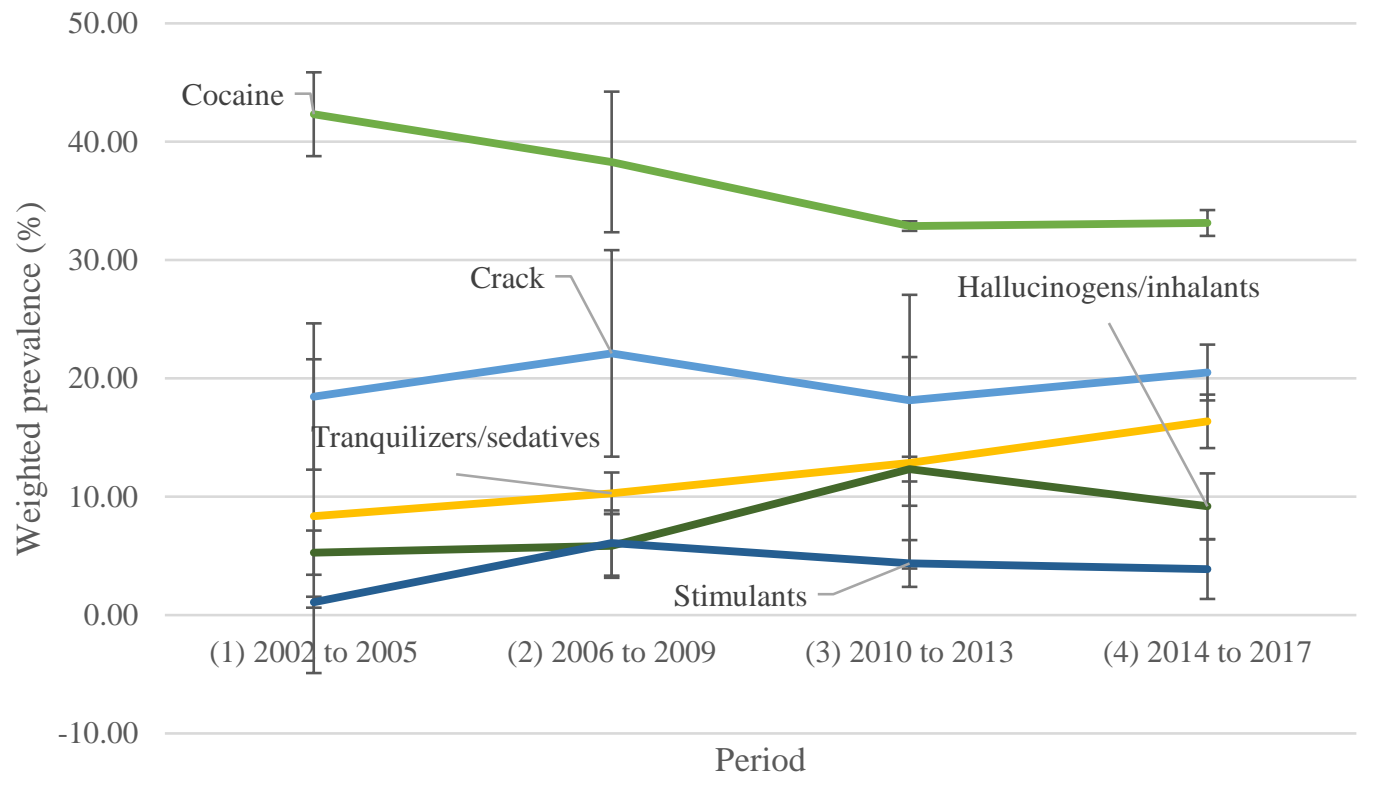

Figure 4.29 | Weighted prevalence of reported past month tranquilizer/sedative, cocaine, crack, hallucinogen/inhalant, and stimulant use among past month heroin-only use group by period $(\mathrm{n}=553)$, NSDUH 2002 to 2017 
Table 4.5 $\mid$ Rao-Scott $\chi^{2}$ analyses for the association of prevalence of each core substance and period for past month heroin-only use group $(n=553)$, NSDUH 2002 to 2017

\begin{tabular}{|c|c|c|c|}
\hline \multirow{2}{*}{\multicolumn{2}{|c|}{ Core substances }} & \multicolumn{2}{|c|}{ Rao-Scott analysis } \\
\hline & & $\chi^{2}(\mathrm{df})$ & $\mathrm{p}$-value \\
\hline \multirow{2}{*}{ Binge alcohol } & Binge use in the past month & \multirow{2}{*}{$\begin{array}{l}3.88 \\
(3)\end{array}$} & \multirow{2}{*}{0.2744} \\
\hline & $\begin{array}{l}\text { Never or 'no' binge use in } \\
\text { past month }\end{array}$ & & \\
\hline \multirow{2}{*}{ Heavy alcohol } & Heavy use in the past month & \multirow{2}{*}{$\begin{array}{c}8.13 \\
(3)\end{array}$} & \multirow{2}{*}{0.0434} \\
\hline & $\begin{array}{l}\text { Never or 'no' heavy use in } \\
\text { past month }\end{array}$ & & \\
\hline \multirow{2}{*}{$\begin{array}{l}\text { Tranquilizers/ } \\
\text { sedatives }\end{array}$} & Used in the past month & \multirow{2}{*}{$\begin{array}{l}2.81 \\
(3)\end{array}$} & \multirow{2}{*}{0.42} \\
\hline & Not used in the past month & & \\
\hline \multirow{2}{*}{ Cigarettes } & Used in the past month & \multirow{2}{*}{$\begin{array}{c}8.26 \\
(3)\end{array}$} & \multirow{2}{*}{0.041} \\
\hline & Not used in the past month & & \\
\hline \multirow{2}{*}{$\begin{array}{l}\text { Other tobacco } \\
\text { products }\end{array}$} & Used in the past month & \multirow{2}{*}{$\begin{array}{l}1.94 \\
(3)\end{array}$} & \multirow{2}{*}{0.58} \\
\hline & Not used in the past month & & \\
\hline \multirow{2}{*}{ Marijuana } & Used in the past month & \multirow{2}{*}{$\begin{array}{l}3.65 \\
(3)\end{array}$} & \multirow{2}{*}{0.30} \\
\hline & Not used in the past month & & \\
\hline \multirow{2}{*}{ Cocaine } & Used in the past month & \multirow{2}{*}{$\begin{array}{l}1.04 \\
(3)\end{array}$} & \multirow{2}{*}{0.79} \\
\hline & Not used in the past month & & \\
\hline \multirow{2}{*}{ Crack } & Used in the past month & \multirow{2}{*}{$\begin{array}{l}0.14 \\
(3)\end{array}$} & \multirow{2}{*}{0.99} \\
\hline & Not used in the past month & & \\
\hline \multirow{2}{*}{$\begin{array}{l}\text { Hallucinogens/ } \\
\text { inhalants }\end{array}$} & Used in the past month & \multirow{2}{*}{$\begin{array}{c}4.32 \\
(3)\end{array}$} & \multirow{2}{*}{0.23} \\
\hline & Not used in the past month & & \\
\hline \multirow{2}{*}{ Stimulants } & Used in the past month & \multirow{2}{*}{$\begin{array}{l}2.45 \\
(3)\end{array}$} & \multirow{2}{*}{0.48} \\
\hline & Not used in the past month & & \\
\hline
\end{tabular}

Abbreviations: $\chi^{2}(\mathrm{df})=$ Chi-square statistic (degrees of freedom)

Note: Bolded $\chi^{2}$ and $p$-values denote statistical significance at $\mathrm{p}<0.05$. 


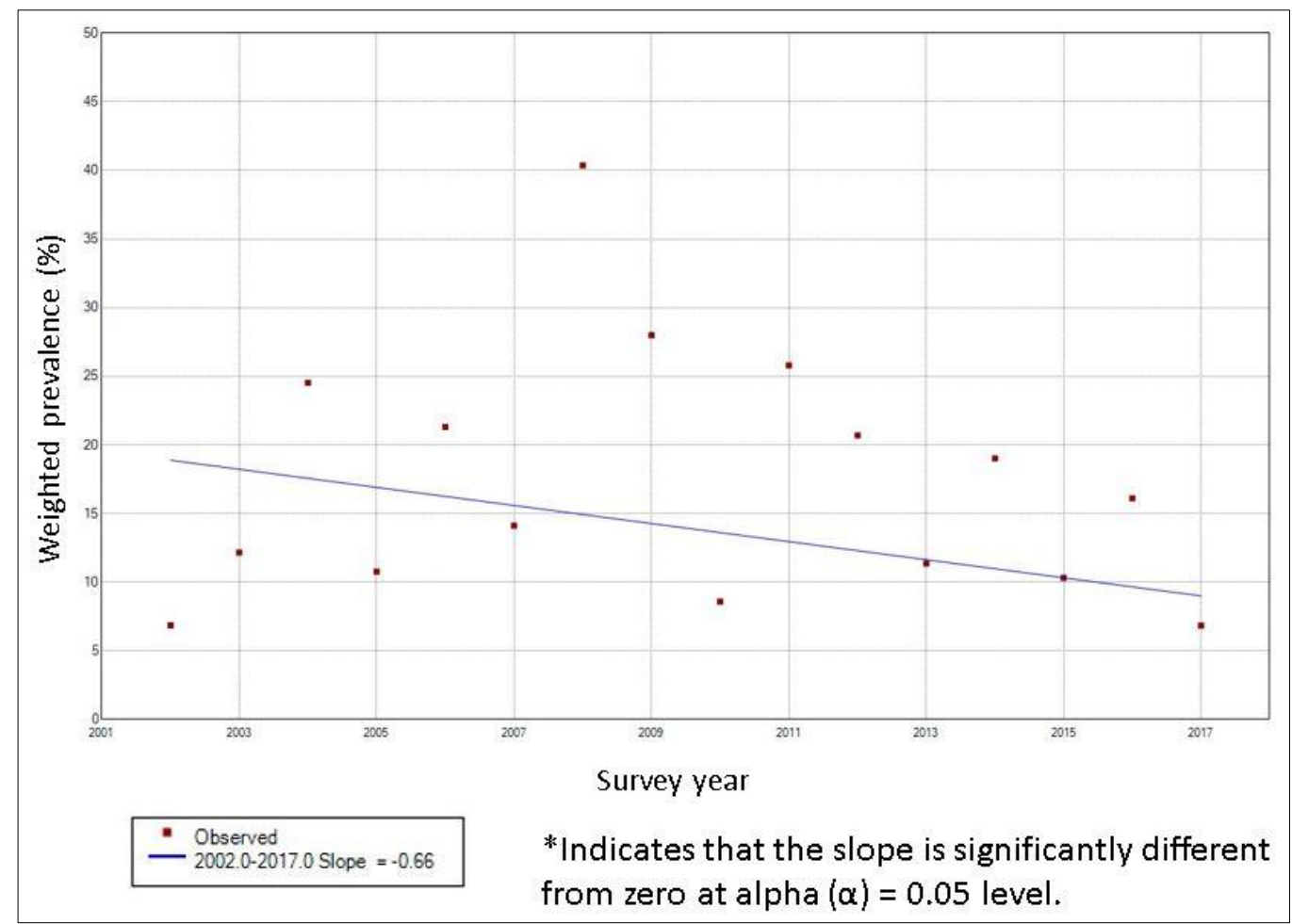

Figure 4.30 $\mid$ Joinpoint analysis of the weighted prevalence of past month heavy alcohol use among past month heroin-only use group $(\mathrm{n}=553)$

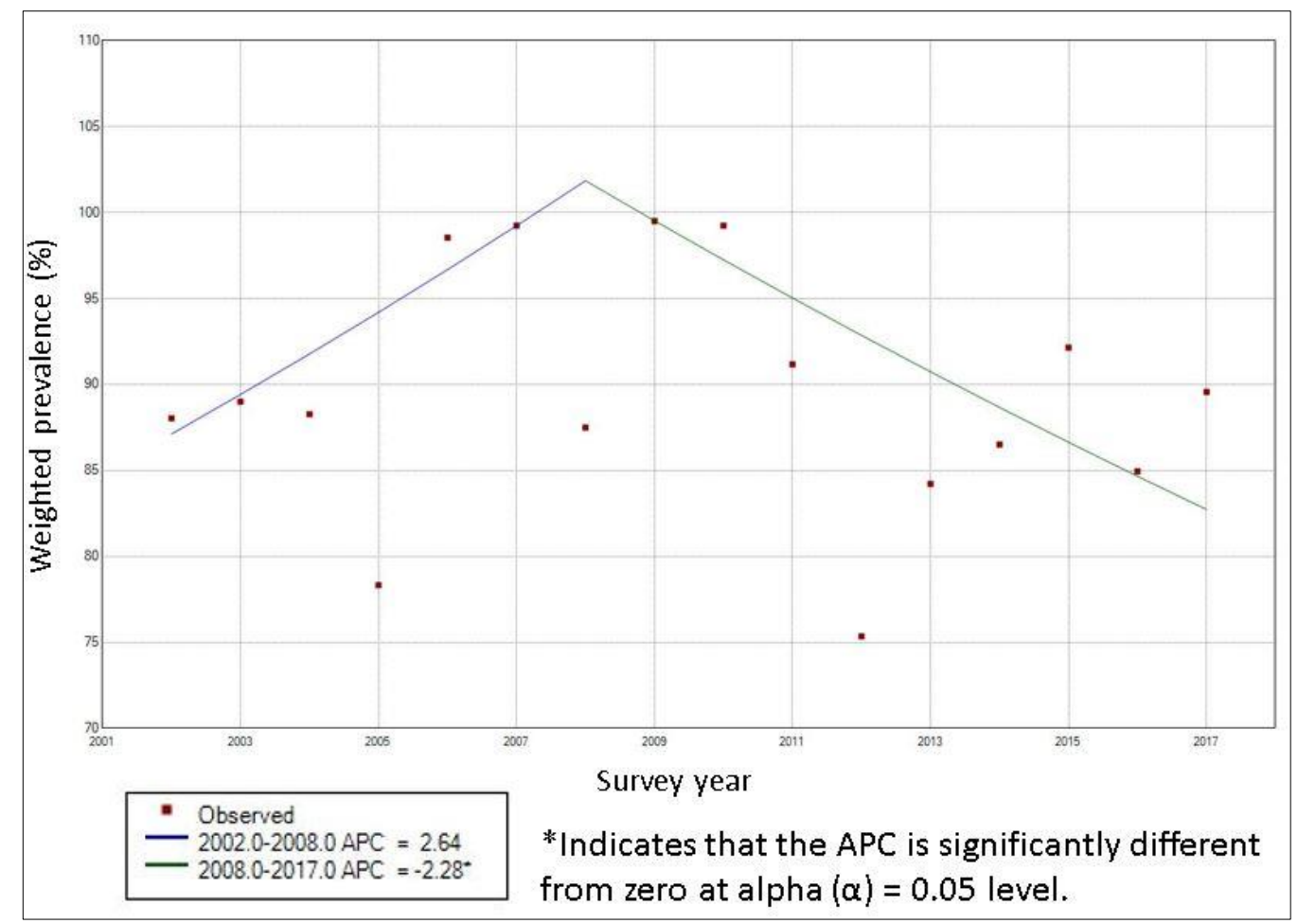

Figure 4.31 | Joinpoint analysis of the weighted prevalence of past month cigarette use among past month heroin-only use group $(\mathrm{n}=553)$ 


\section{Past month combination opioid use group}

Figures 4.32 to 4.34 show the weighted prevalence of all past month core substance use from period 1 to 4 . The substances that showed increases in reported prevalence were tranquilizers/sedatives and stimulants. It should be noted that the prevalence of most substances for all periods among the past month combination opioid use groups exceeded those of the past month PPR-only use group. (Graphs comparing the weighted prevalence of core substance use stratified by opioid use group and period are provided in Supplemental Graphs (SG) 4.1a-d, 4.2a-d, 4.3a-d, and 4.4a-d in

\section{Appendix B).}

As shown in Table 4.6, $\chi^{2}$ analysis identified three core substances whose prevalence were significantly associated with period: $\operatorname{crack}\left[\chi^{2}(\mathrm{df}),(\mathrm{p}\right.$-value): $8.88(3)$, (0.031)], hallucinogens/inhalants [ $\chi^{2}(\mathrm{df}),(\mathrm{p}$-value): $10.58(3),(0.014)]$, and stimulants $\left[\chi^{2}\right.$ (df), (p-value): 26.58 (3), $(<0.0001)]$. Joinpoint regression results for the prevalence of these three substances are shown in Figures 4.35 through 4.37. Although no joinpoints were modeled, the trends in the prevalence of the reported past month use of crack [m (pvalue): 0.46 (0.004)] and stimulants [m (p-value): $1.78(0.004)]$ were significant and positive in this population. However, the trend in the prevalence of past month hallucinogen/inhalant use showed two joinpoints: 2006 and 2011, which indicated two trends in the prevalence of past month hallucinogen/inhalant use. From 2002 to 2006, there was a decrease in the prevalence of reported past month hallucinogen/inhalant use, followed by an increase from 2006 to 2011, although neither of these trends were significant. Nevertheless, the prevalence trend from 2011 to 2017 was significant yet 
negative [m (p-value): -3.99 (0.013)]. (For all joinpoint results for this opioid use group, see ST 4.12a-c in Appendix A). 


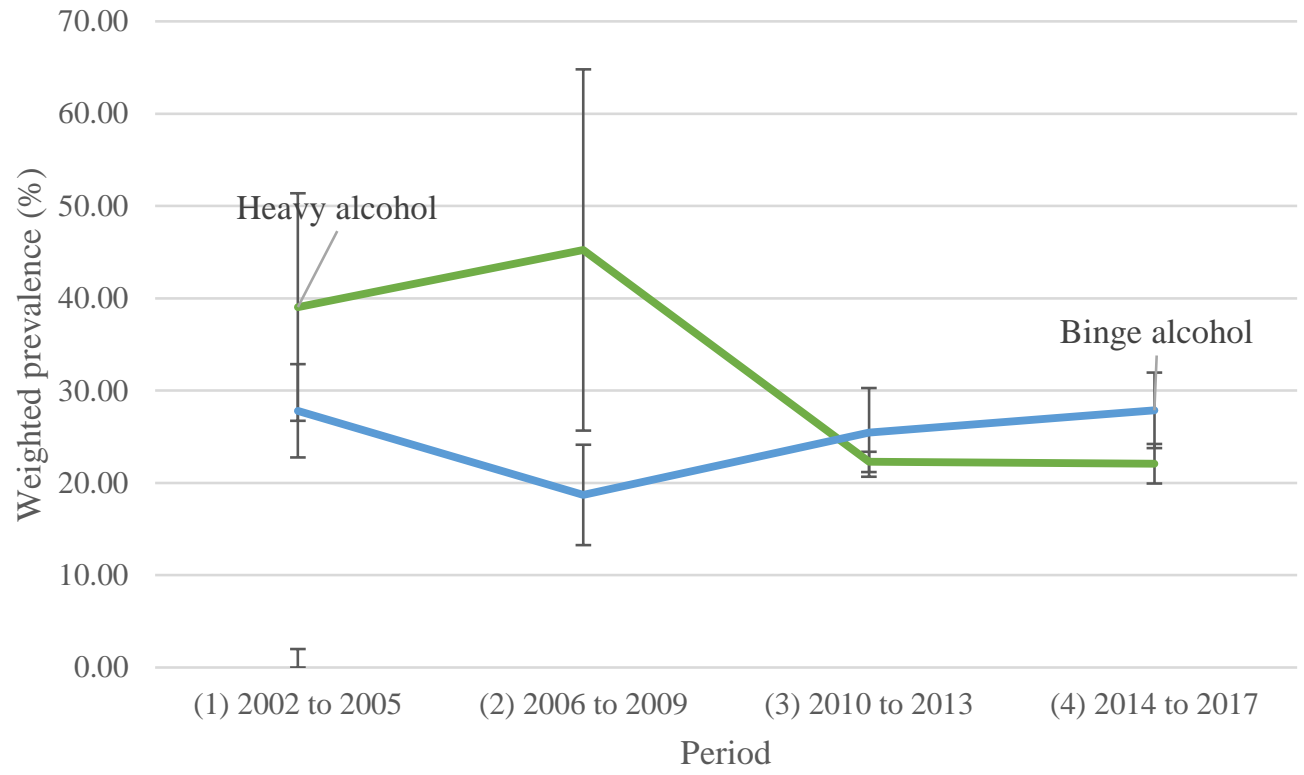

Figure 4.32 $\mid$ Weighted prevalence of all levels of past month alcohol drinking among past month combination opioid use group by period $(\mathrm{n}=535)$, NSDUH 2002 to 2017

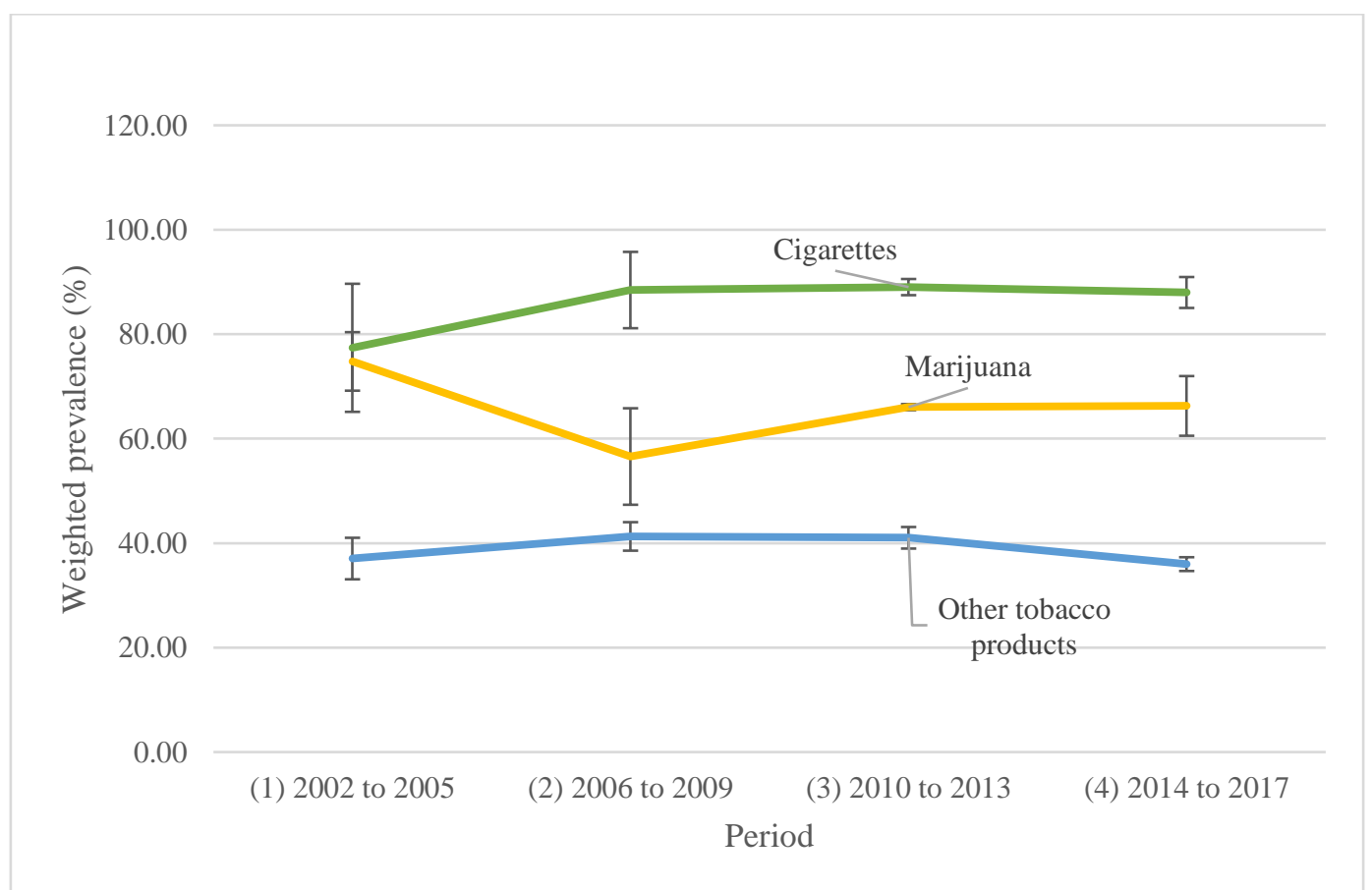

Figure 4.33 $\mid$ Weighted prevalence of reported past month cigarette smoking, other tobacco use, and marijuana use among past month combination opioid use group by period $(n=535)$, NSDUH 2002 to 2017 


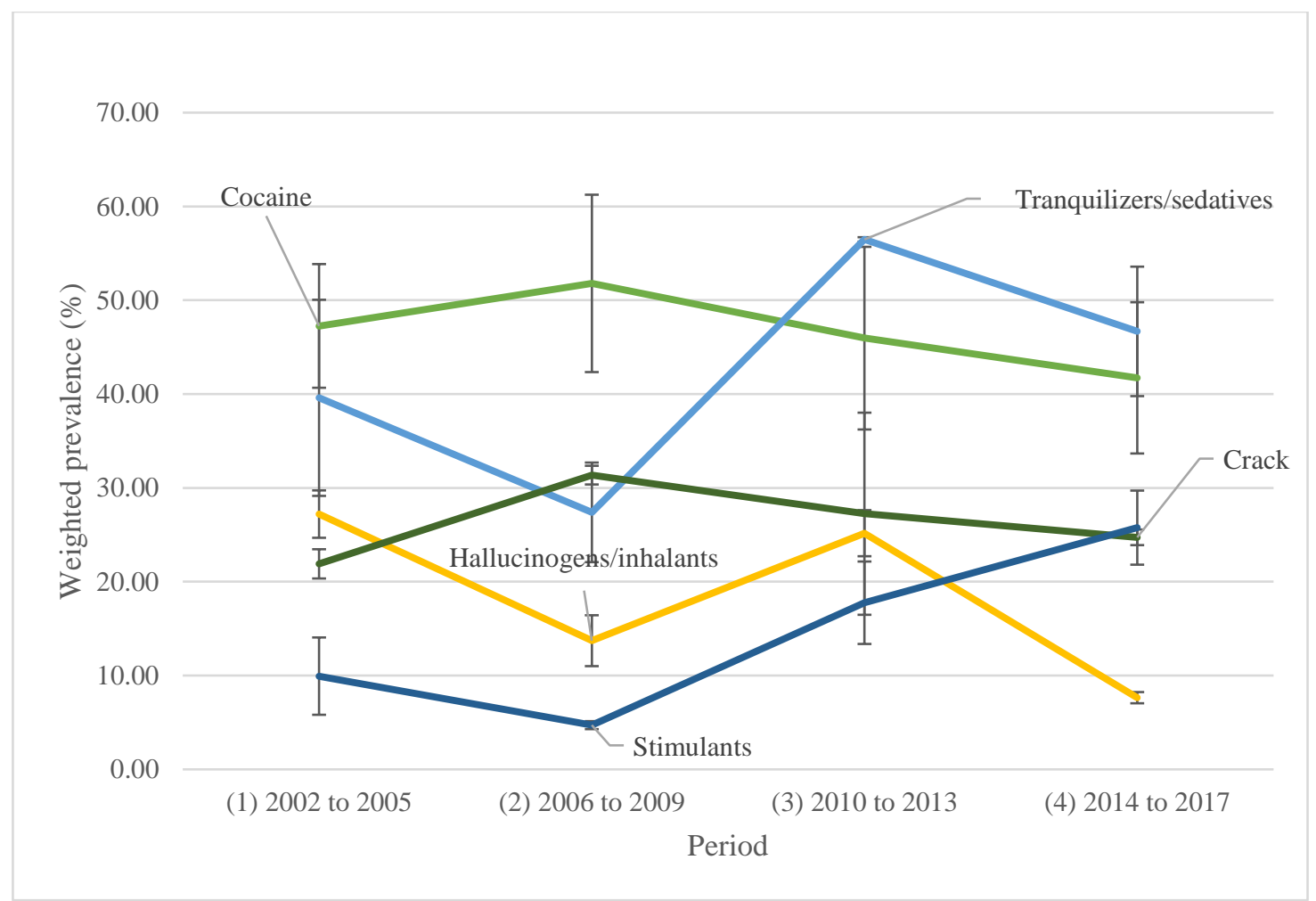

Figure 4.34 | Weighted prevalence of reported past month tranquilizer/sedative, cocaine, crack, hallucinogen/inhalant, and stimulant use among past month combination opioid use group by period $(n=535)$, NSDUH 2002 to 2017 
Table 4.6 $\mid$ Rao-Scott $\chi^{2}$ analyses for the association of prevalence of each core substance and period for past month combination opioid use group $(n=535)$, NSDUH 2002 to 2017

\begin{tabular}{|c|c|c|c|}
\hline \multirow{2}{*}{\multicolumn{2}{|c|}{ Core substances }} & \multicolumn{2}{|c|}{ Rao-Scott analysis } \\
\hline & & $\chi^{2}(\mathrm{df})$ & n-value \\
\hline \multirow{2}{*}{ Binge alcohol } & Binge use in the past month & \multirow{2}{*}{$\begin{array}{c}2.56 \\
(3)\end{array}$} & \multirow{2}{*}{0.4636} \\
\hline & $\begin{array}{c}\text { Never or 'no' binge use in } \\
\text { past month }\end{array}$ & & \\
\hline \multirow{2}{*}{ Heavy alcohol } & Heavy use in the past month & \multirow{2}{*}{$\begin{array}{l}5.00 \\
(3)\end{array}$} & \multirow{2}{*}{0.1718} \\
\hline & $\begin{array}{l}\text { Never or 'no' heavy use in } \\
\text { past month }\end{array}$ & & \\
\hline \multirow{2}{*}{$\begin{array}{l}\text { Tranquilizers/ } \\
\text { sedatives }\end{array}$} & Used in the past month & \multirow{2}{*}{$\begin{array}{l}4.73 \\
(3)\end{array}$} & \multirow{2}{*}{0.19} \\
\hline & Not used in the past month & & \\
\hline \multirow{2}{*}{ Cigarettes } & Used in the past month & \multirow{2}{*}{$\begin{array}{l}1.45 \\
(3)\end{array}$} & \multirow{2}{*}{0.69} \\
\hline & Not used in the past month & & \\
\hline \multirow{2}{*}{$\begin{array}{l}\text { Other tobacco } \\
\text { products }\end{array}$} & Used in the past month & \multirow{2}{*}{$\begin{array}{c}3.86 \\
(3)\end{array}$} & \multirow{2}{*}{0.28} \\
\hline & Not used in the past month & & \\
\hline \multirow{2}{*}{ Marijuana } & Used in the past month & \multirow{2}{*}{$\begin{array}{c}2.84 \\
(3)\end{array}$} & \multirow{2}{*}{0.42} \\
\hline & Not used in the past month & & \\
\hline \multirow{2}{*}{ Cocaine } & Used in the past month & \multirow{2}{*}{$\begin{array}{l}1.66 \\
(3)\end{array}$} & \multirow{2}{*}{0.65} \\
\hline & Not used in the past month & & \\
\hline \multirow{2}{*}{ Crack } & Used in the past month & \multirow{2}{*}{$\begin{array}{c}8.88 \\
(3)\end{array}$} & \multirow{2}{*}{0.031} \\
\hline & Not used in the past month & & \\
\hline \multirow{2}{*}{$\begin{array}{l}\text { Hallucinogens/ } \\
\text { inhalants }\end{array}$} & Used in the past month & \multirow{2}{*}{$\begin{array}{c}10.58 \\
(3)\end{array}$} & \multirow{2}{*}{0.014} \\
\hline & Not used in the past month & & \\
\hline \multirow{2}{*}{ Stimulants } & Used in the past month & \multirow{2}{*}{$\begin{array}{l}26.58 \\
(3)\end{array}$} & \multirow{2}{*}{$<0.0001$} \\
\hline & Not used in the past month & & \\
\hline
\end{tabular}

Abbreviations: $\chi^{2}(\mathrm{df})=$ Chi-square statistic (degrees of freedom)

Note: Bolded $\chi^{2}$ and $p$-values denote statistical significance at $\mathrm{p}<0.05$. 


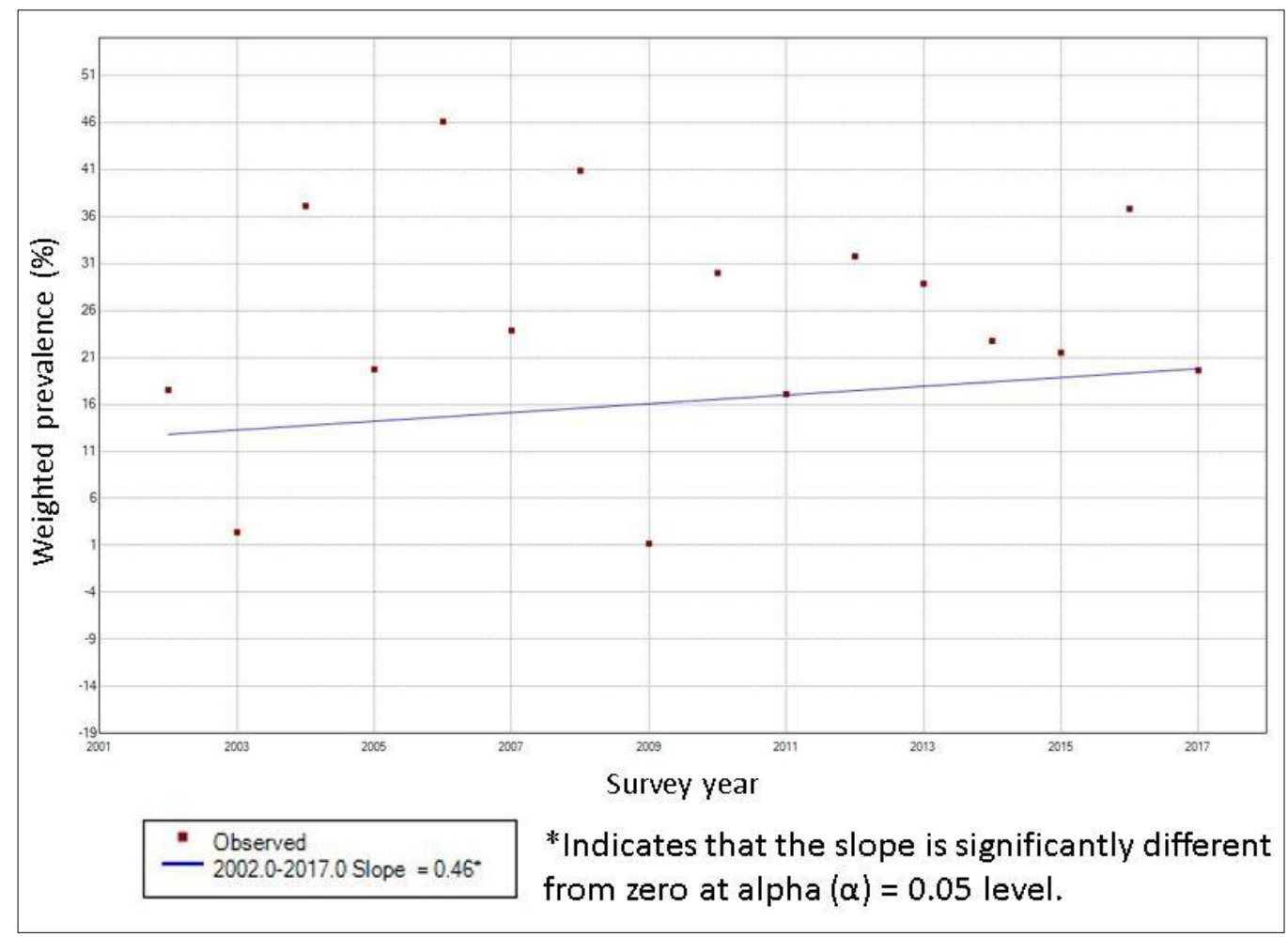

Figure 4.35 | Joinpoint analysis of the weighted prevalence of past month crack use among past month combination opioid use group $(n=535)$

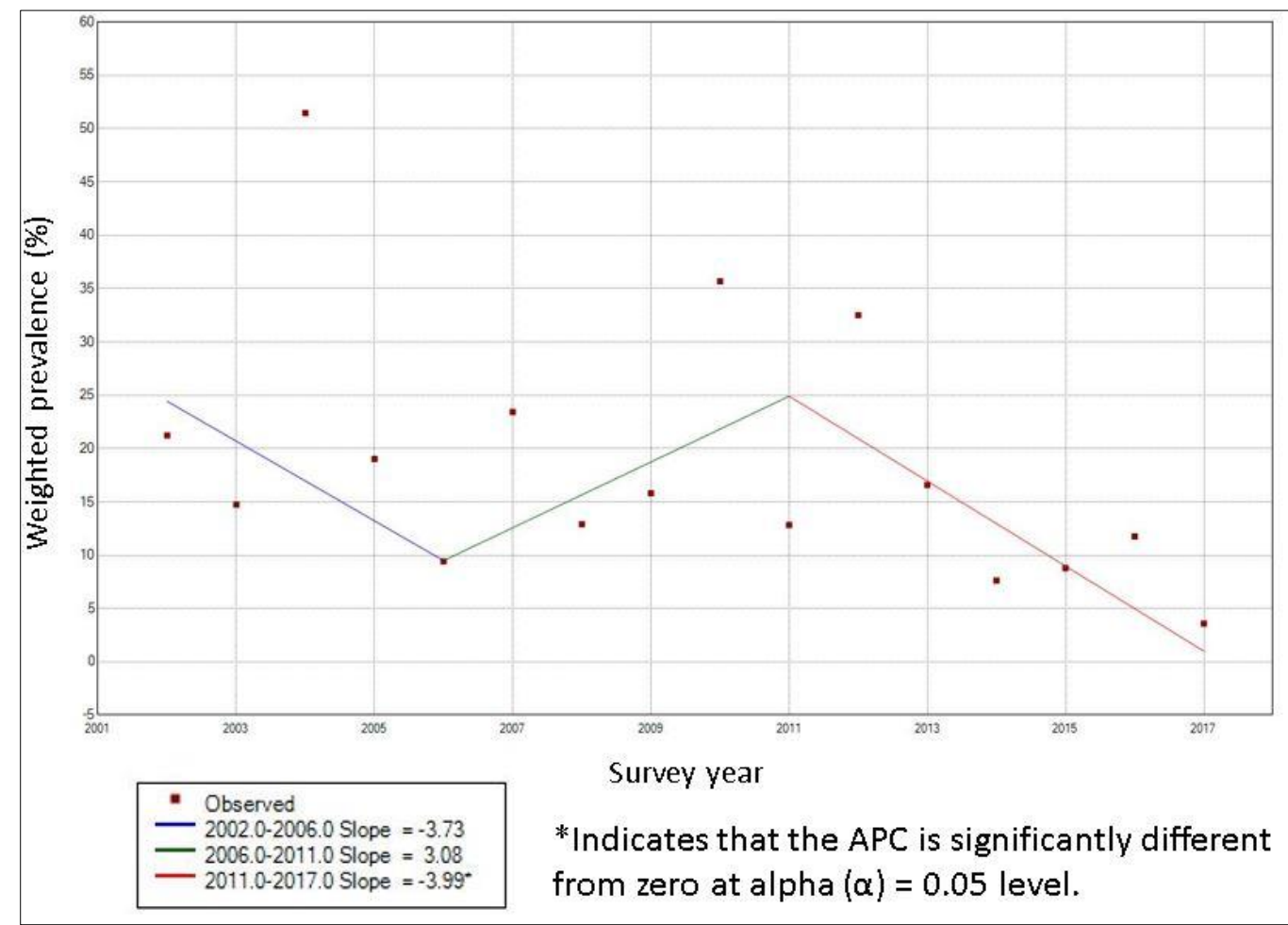

Figure 4.36 | Joinpoint analysis of the weighted prevalence of past month hallucinogen/inhalant use among past month combination opioid use group $(n=535)$ 


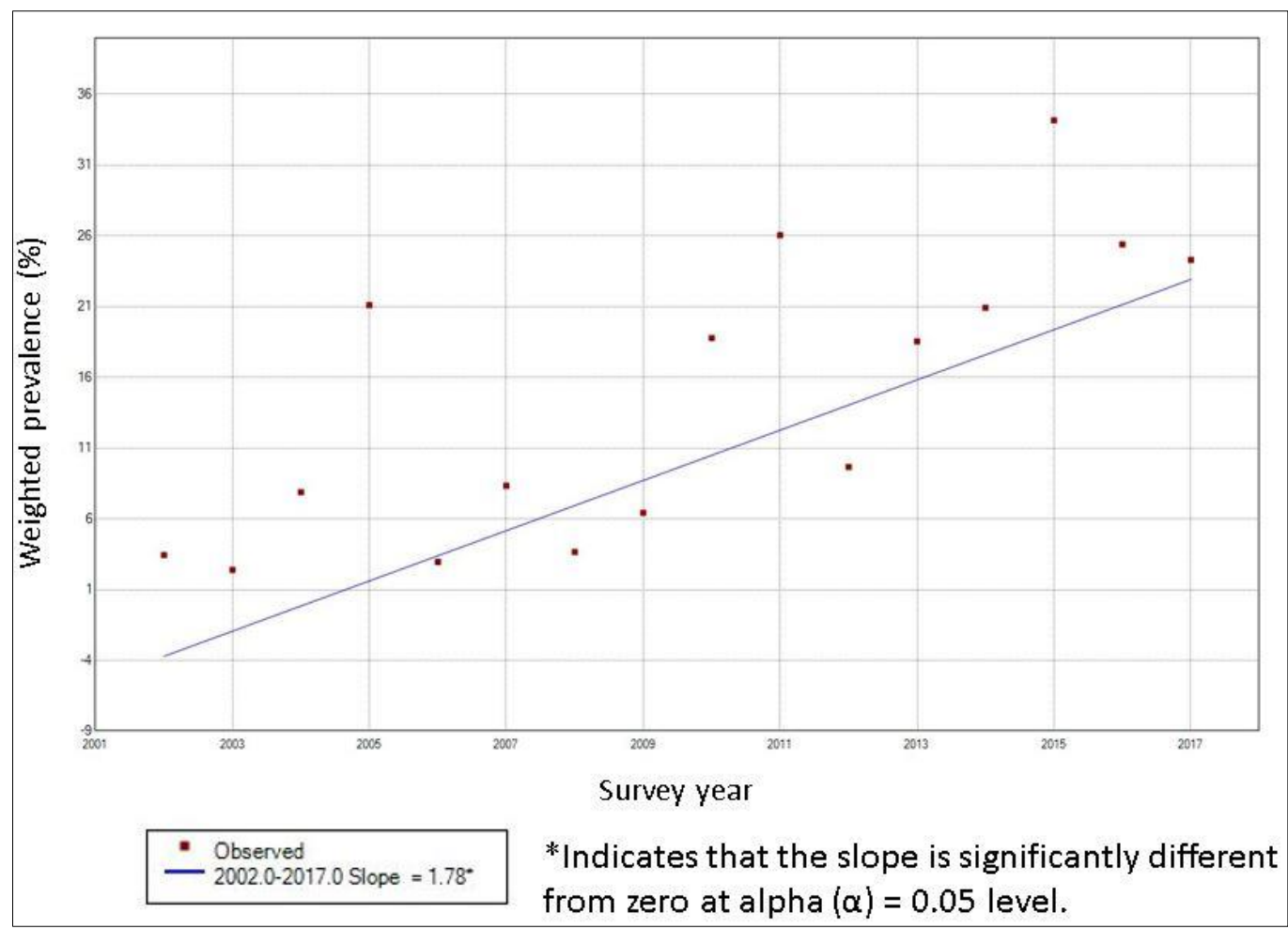

Figure 4.37 | Joinpoint analysis of the weighted prevalence of past month stimulant use among past month combination opioid use group $(n=535)$ 


\begin{abstract}
Aim 2 Results
Subaim 2.1: To identify the latent class variable of polydrug use in PWUO using the past-month use variables of the 11 core substance indicators (pain relievers, heroin, heavy alcohol, tranquilizers/sedatives, cigarettes, other tobacco products, marijuana, cocaine, crack, hallucinogens/inhalants, and stimulants).
\end{abstract}

Four primary subpopulations were identified using latent class analysis. This final number of classes were enumerated primarily using the results of the BIC and entropy fit statistics. Aim 1 results were also used to confirm likely patterns of use among Classes whose primary opioid use population were akin to the opioid use groups. Figure 4.38 compares the three information criteria (i.e., AIC, BIC, ssBIC) used to guide final class enumeration. As shown in Figure 4.38, all IC statistics were extremely comparable, with the BIC being the most conservative measure. And although all ICs continued to decrease beyond the four-class model, the decline was extremely modest. Entropy values were also used to determine the final class size; Figure 4.39 shows the plotted entropy values for models fitting two to eight polydrug use classes. (For all fit statistic results, see ST 4.14 in Appendix A). 


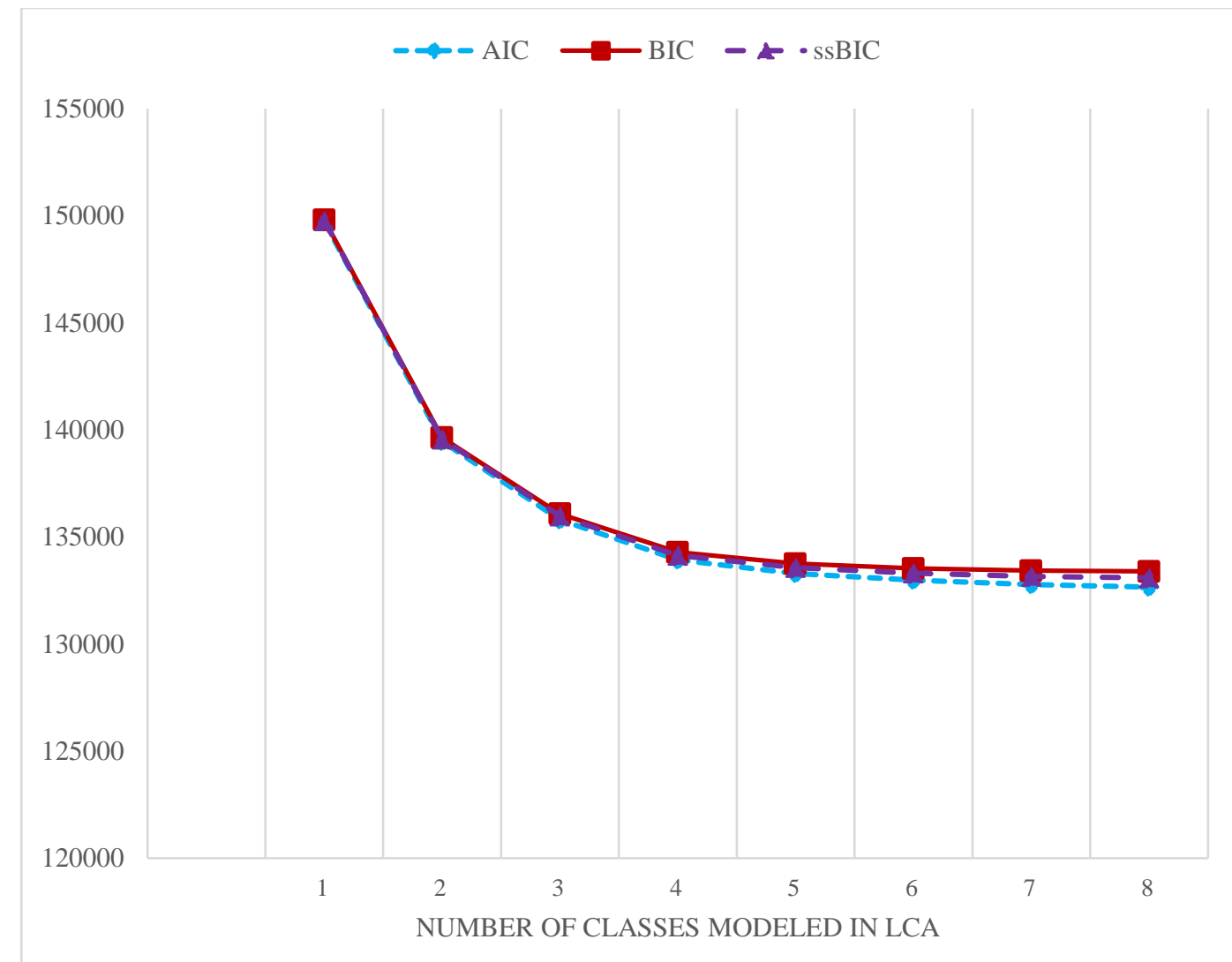

Figure 4.38 | Information criteria comparisons for one to eight polydrug use classes in PWUO $(n=16,985)$, NSDUH 2002 to 2017

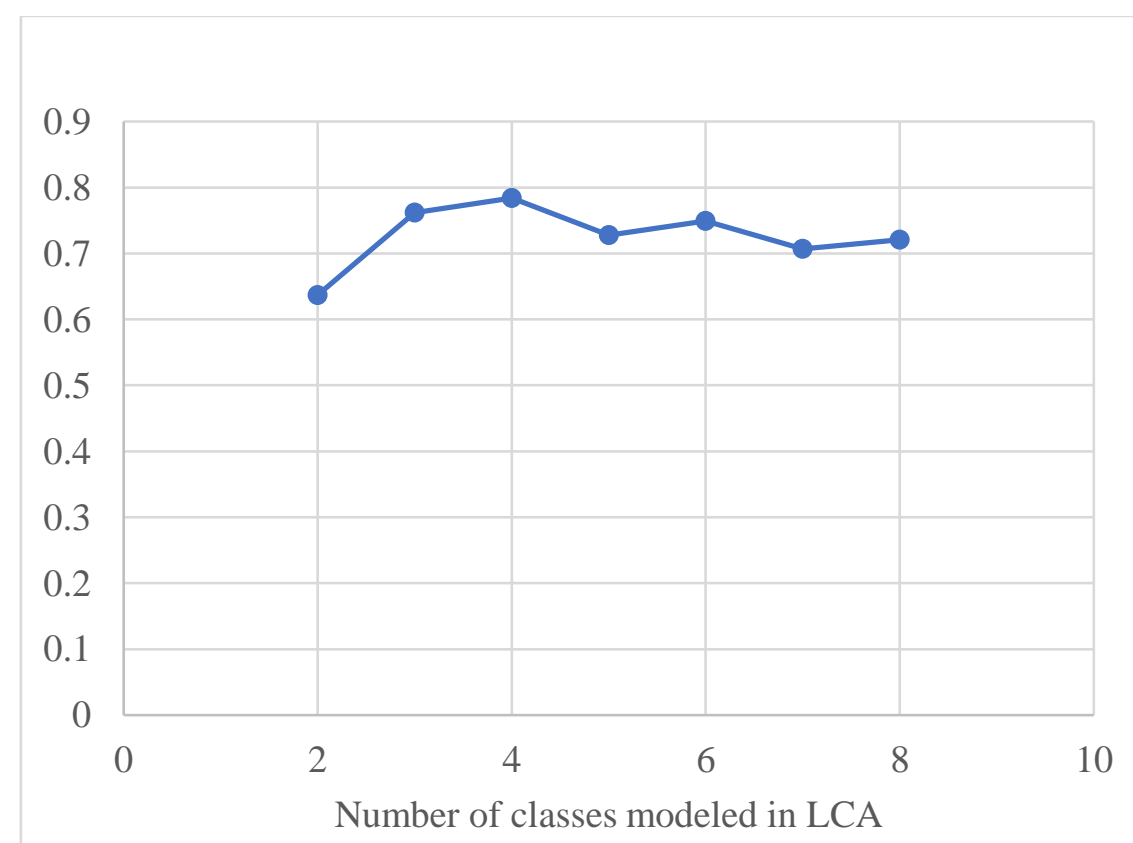

Figure 4.39 | Entropy comparisons for two to eight polydrug use classes in PWUO ( $n=16,985)$, NSDUH 2002 to 2017 
Additionally, to assess whether a five-class model yielded a better fit, endorsement probabilities were graphed and compared to the four-class model. It was determined that Class 5 yielded an additional class that was not logical based on the prevalence results from Aim 1. And while most of the other classes in the five-class model remained comparable to the four-class model, the extra class from the five-class model included a combination opioid subpopulation that had $100 \%$ endorsement probabilities for cocaine and crack, which was not supported by Aim 1 results.

Table 4.7 shows the sample size and weighted prevalence of the four polydrug latent classes, while Table 4.8 shows all endorsement probabilities for past month core substance use among each latent class. Figure $\mathbf{4 . 4 0}$ shows the profiles of polydrug use behavior using a four-class model. Classes were named based on substance use categories that exhibited higher endorsement probabilities. It should be noted that 'cigarettes' were not included in the name of any latent class because of its high endorsement probability for all latent classes. Class 1 (mainly PPR) was characterized by PPR use with low probabilities of endorsing all other core substances, making up approximately $44.1 \%$ of all PWUO. Class 2 was characterized by high endorsement probabilities for PPR, heavy alcohol, prescription tranquilizers/sedatives, prescription stimulants, and marijuana use; it comprised $42.6 \%$ of all PWUO. Class 3 was characterized by heroin, marijuana, and other illicit substances (i.e., cocaine and crack) and made up $3.6 \%$ of all polydrug latent classes. Finally, Class 4 was characterized by combination opioid use and high endorsement probabilities for all other core substances and made up $9.6 \%$ of all polydrug latent classes. 
Table 4.7 | Overall size and weighted prevalence of the latent polydrug use subpopulations among PWUO $(n=16,985)$, NSDUH 2002 to 2017

\begin{tabular}{|c|c|c|c|c|c|}
\hline Polydrug latent class & \multirow{2}{*}{$\mathbf{n}$} & $\boldsymbol{*}$ & $\mathbf{S E}$ & \multicolumn{2}{|c|}{$\mathbf{9 5 \%}$ CI } \\
\hline 1: mainly PPR & 5817 & 44.13 & 0.57 & 42.33 & 45.93 \\
\hline $\begin{array}{c}\text { 2: PPR - heavy alcohol - benzo - } \\
\text { stimulant - marijuana }\end{array}$ & 8709 & 42.64 & 0.81 & 40.06 & 45.22 \\
\hline 3: Heroin - marijuana - illicit & 553 & 3.61 & 0.16 & 3.10 & 4.12 \\
\hline 4ubstances & 1906 & 9.62 & 0.34 & 8.55 & 10.70 \\
\hline
\end{tabular}

Abbreviations: $\mathrm{n}=$ total number of survey respondents; $\%=$ weighted percentage; $\mathrm{SE}=$ standard error; $95 \% \mathrm{CI}=95 \%$ confidence interval; $\mathrm{LL}=$ lower limit; $\mathrm{UL}=$ upper limit

Table 4.8 Endorsement probabilities for each core substance by latent polydrug class ( $\mathrm{n}=16,985)$, NSDUH 2002 to 2017

\begin{tabular}{|c|c|c|c|c|}
\hline & \multicolumn{3}{|c|}{ Polydrug latent classes among PWUO } \\
\hline $\begin{array}{c}\text { Substances used in the } \\
\text { past 30-days }\end{array}$ & 1: mainly PPR & $\begin{array}{c}\text { 2: PPR - heavy } \\
\text { alcohol - benzo - } \\
\text { stimulant - drugs } \\
\text { - marijuana }\end{array}$ & $\begin{array}{c}\text { 3: Heroin - } \\
\text { marijuana - } \\
\text { illicit } \\
\text { substances }\end{array}$ & $\begin{array}{c}\text { Combination } \\
\text { opioid - } \\
\text { polydrug }\end{array}$ \\
\hline PPR & 1 & 1 & 0.022 & 0.999 \\
\hline Heroin & 0.001 & 0.037 & 1 & 0.159 \\
\hline Heavy alcohol & 0.088 & 0.385 & 0.164 & 0.516 \\
\hline Cigarettes & 0.299 & 0.814 & 0.887 & 0.862 \\
\hline Tranquilizers/sedatives & 0.092 & 0.291 & 0.134 & 0.459 \\
\hline Stimulants & 0.014 & 0.121 & 0.039 & 0.217 \\
\hline Marijuana & 0.096 & 0.669 & 0.478 & 0.75 \\
\hline Cocaine & 0.008 & 0.025 & 0.354 & 1 \\
\hline Crack & 0 & 0 & 0.199 & 0.346 \\
\hline Hallucinogens/inhalants & 0.009 & 0.095 & 0.085 & 0.241 \\
\hline
\end{tabular}




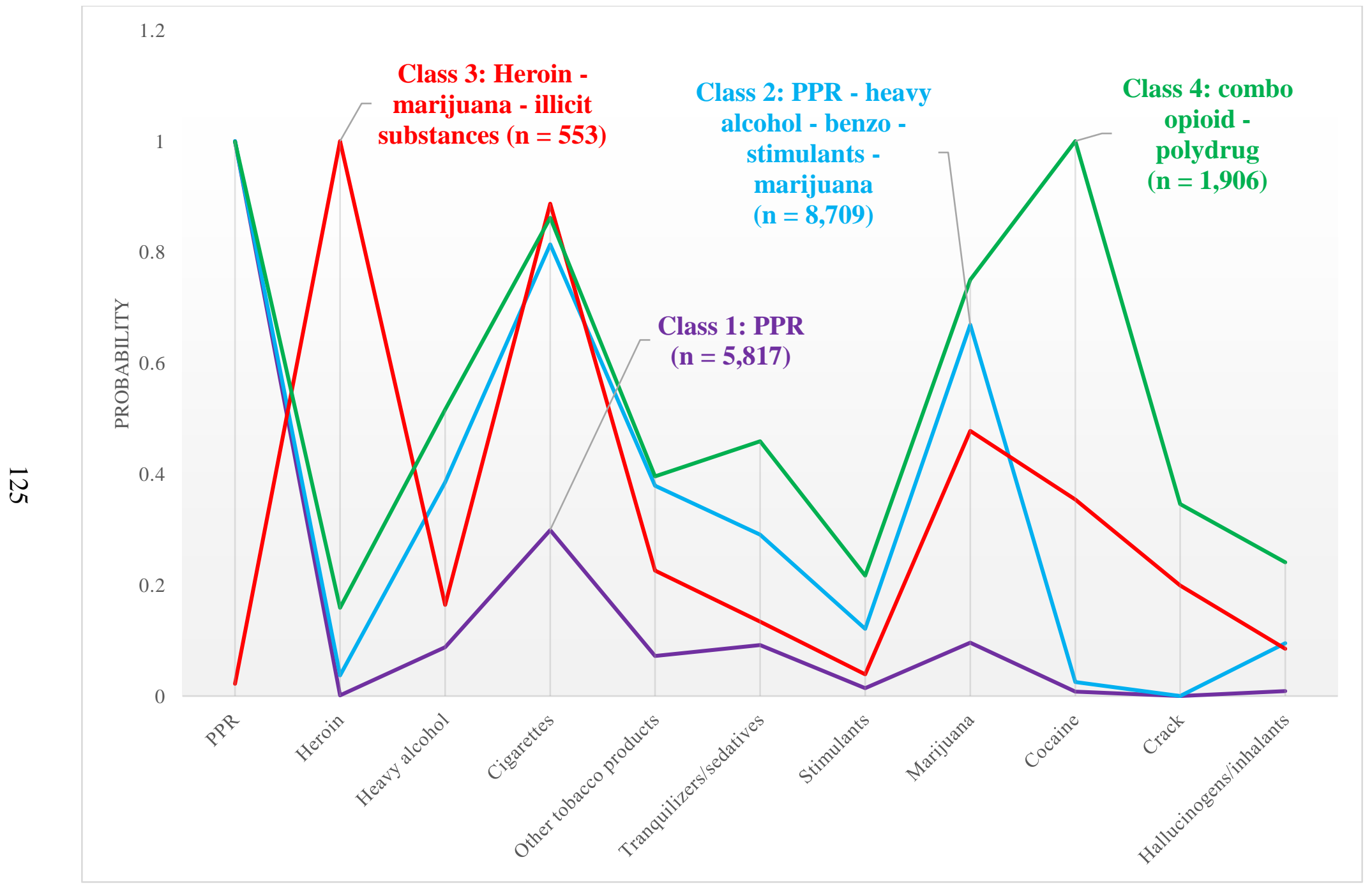

Figure 4.40 | Four Class Model Endorsement Probability Plot ( $\mathrm{n}=16,985)$, NSDUH 2002 to 2017 


\section{Subaim 2.2: To determine whether the prevalence of each latent class has remained constant}

\section{Longitudinal trends of the weighted prevalence of the polydrug latent classes}

Figure 4.41 shows the longitudinal trend of each polydrug latent class from period 1 to 4 . While the overall trend in the prevalence of these latent classes over time remains relatively constant, Class 3 (heroin-marijuana-illicit substance), appear to be increasing over time. Chi-square results found in Table 4.9 also indicated a significant association between period and the prevalence of the latent classes $\left[\chi^{2}(\mathrm{df}),(\mathrm{p}\right.$-value): $42.06(9),(<0.0001)]$.

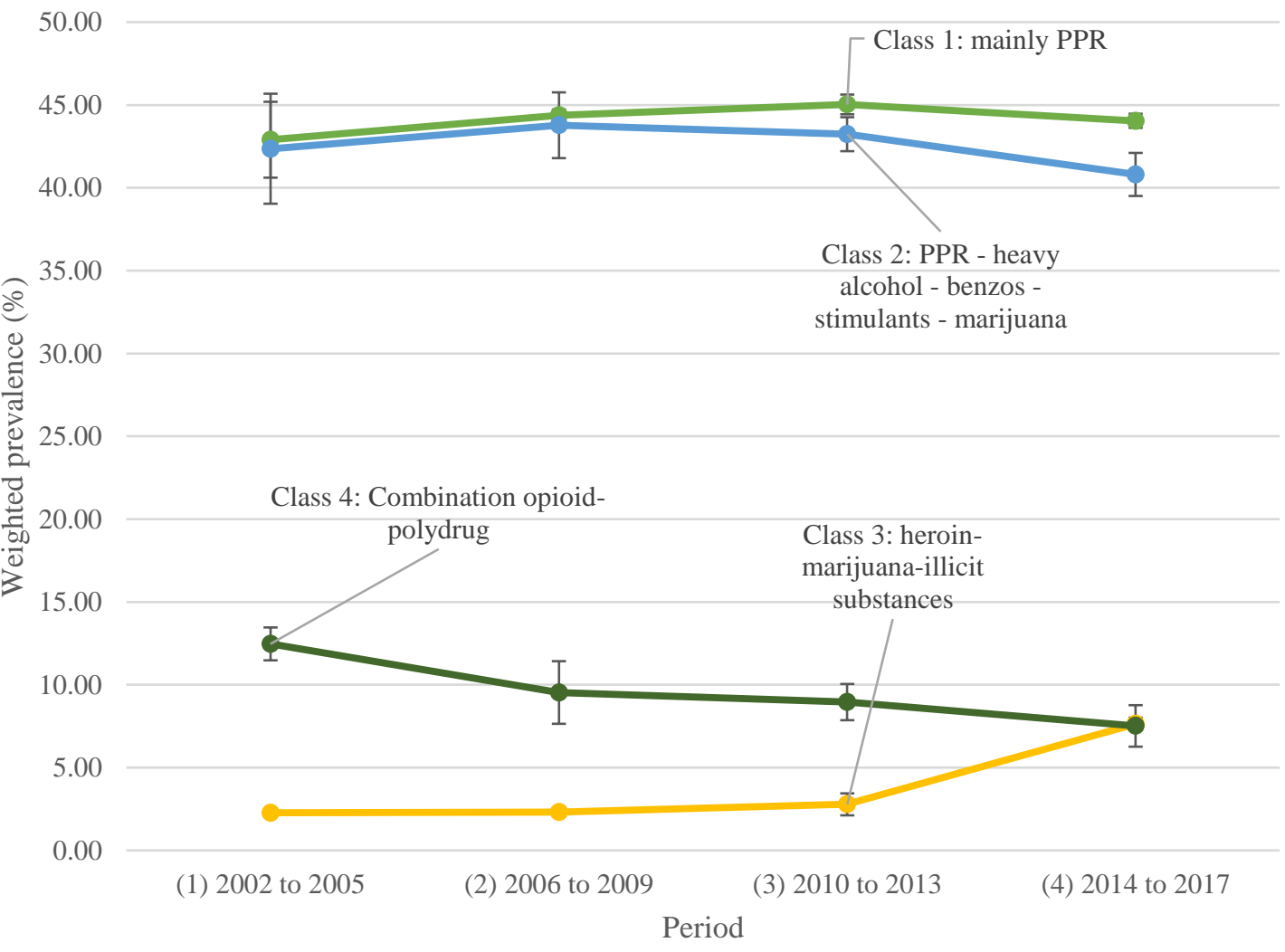

Figure 4.41 | Weighted prevalence of polydrug latent classes by period $(n=16,985)$, NSDUH 2002 to 2017 
Table 4.9 | Weighted prevalence of polydrug latent classes in PWUO by period $(n=16,985)$, NSDUH 2002 to 2017

\begin{tabular}{|c|c|c|c|c|c|c|c|c|c|c|c|c|c|c|}
\hline \multirow{3}{*}{ Sample } & \multicolumn{12}{|c|}{ Period } & \multicolumn{2}{|c|}{$\begin{array}{c}\text { Rao-Scott } \\
\text { Analyses }\end{array}$} \\
\hline & \multicolumn{3}{|c|}{ (1) 2002 to 2005} & \multicolumn{3}{|c|}{ (2) 2006 to 2009} & \multicolumn{3}{|c|}{ (3) 2010 to 2013} & \multicolumn{3}{|c|}{ (4) 2014 to 2017} & $\begin{array}{c}\chi^{2} \\
(\mathrm{df})\end{array}$ & p-value \\
\hline & $\mathrm{n}$ & $\%^{\mathrm{a}}$ & SE & $\mathrm{n}$ & $\%^{\mathrm{a}}$ & SE & $\mathrm{n}$ & $\%^{\mathrm{a}}$ & SE & $\mathrm{n}$ & $\%^{\mathrm{a}}$ & SE & & \\
\hline $\begin{array}{c}\text { 1: mainly PPR } \\
(\mathrm{n}=5,817)\end{array}$ & 1455 & 42.90 & 2.29 & 1678 & 44.38 & 0.34 & 1513 & 45.03 & 0.60 & 1171 & 44.04 & 0.42 & & \\
\hline $\begin{array}{c}\text { 2: PPR - heavy } \\
\text { alcohol - benzo - } \\
\text { stimulant - } \\
\text { marijuana } \\
(n=8,709)\end{array}$ & 2269 & 42.36 & 3.32 & 2663 & 43.78 & 1.98 & 2286 & 43.23 & 1.03 & 1491 & 40.81 & 1.30 & & \\
\hline $\begin{array}{c}\text { 3: Heroin - } \\
\text { marijuana - } \\
\text { illicit substances } \\
\quad(n=553)\end{array}$ & 80 & 2.27 & 0.15 & 88 & 2.31 & 0.25 & 144 & 2.78 & 0.66 & 241 & 7.64 & 0.38 & $\begin{array}{c}42.06 \\
(9)\end{array}$ & $<0.0001$ \\
\hline $\begin{array}{l}\text { 4: Combination } \\
\text { opioid - } \\
\text { polydrug } \\
(\mathbf{n}=1,906)\end{array}$ & 653 & 12.47 & 0.99 & 558 & 9.53 & 1.89 & 416 & 8.96 & 1.09 & 279 & 7.52 & 1.25 & & \\
\hline
\end{tabular}

Abbreviations: $\mathrm{n}=$ total number of survey respondents; $\%=$ weighted column percentage; $\mathrm{SE}=$ standard error; $\chi^{2}(\mathrm{df})=\mathrm{Chi}$-square statistic (degrees of freedom)

Note: $95 \%$ confidence intervals for all reported prevalence indicated reliable measures

Note: Bolded $\chi^{2}$ and $p$-values denote statistical significance at $\mathrm{p}<0.05$.

${ }^{a}$ Weighted prevalence was based on the total number of survey participants within each period 
Joinpoint regression results for the change in prevalence of each latent class indicated that the trajectory for Class changed over time. Figures $\mathbf{4 . 4 2}$ through $\mathbf{4 . 4 5}$ show the trend lines for the prevalence of Class 1 (mainly PPR), Class 2 (PPR-heavy alcohol-benzo-stimulant-marijuana), Class 3 (heroin-marijuana-illicit substance), and Class 4 (combination opioid-polydrug), respectively. For Class 1 (mainly PPR), two joinpoints were detected: 2009 and 2012. The overall trajectory of the prevalence of Class 1 (mainly PPR) from 2002 to 2012 was positive, although not significant; however, from 2012 to 2017, there was a significant decrease in the prevalence of Class 1 (mainly PPR) [m (p-value): -0.71 (<0.001)]. For Class 2, one joinpoint was assessed: 2009. From 2002 to 2009, the prevalence of Class 2 significantly increased [m (p-value): 0.87 (0.029)]. However, a significant decrease in the trend in prevalence for Class 2 occurred from 2009 to 2017 [m (p-value): $-0.73(<0.001)]$. While the overall trend in the prevalence of Class 3 was positive, one joinpoint in 2010 was detected, with a significant positive increase from 2010 to 2017 [m (p-value): 1.06 (<0.001)]. Finally, although no joinpoints were found in the trajectory of the prevalence of Class 4 , its overall trend from 2002 to 2017 significantly decreased [m (p-value): -0.32 (0.001)]. (Full results of the joinpoint regression may be found in ST 4.15 through 4.18 in Appendix A). 


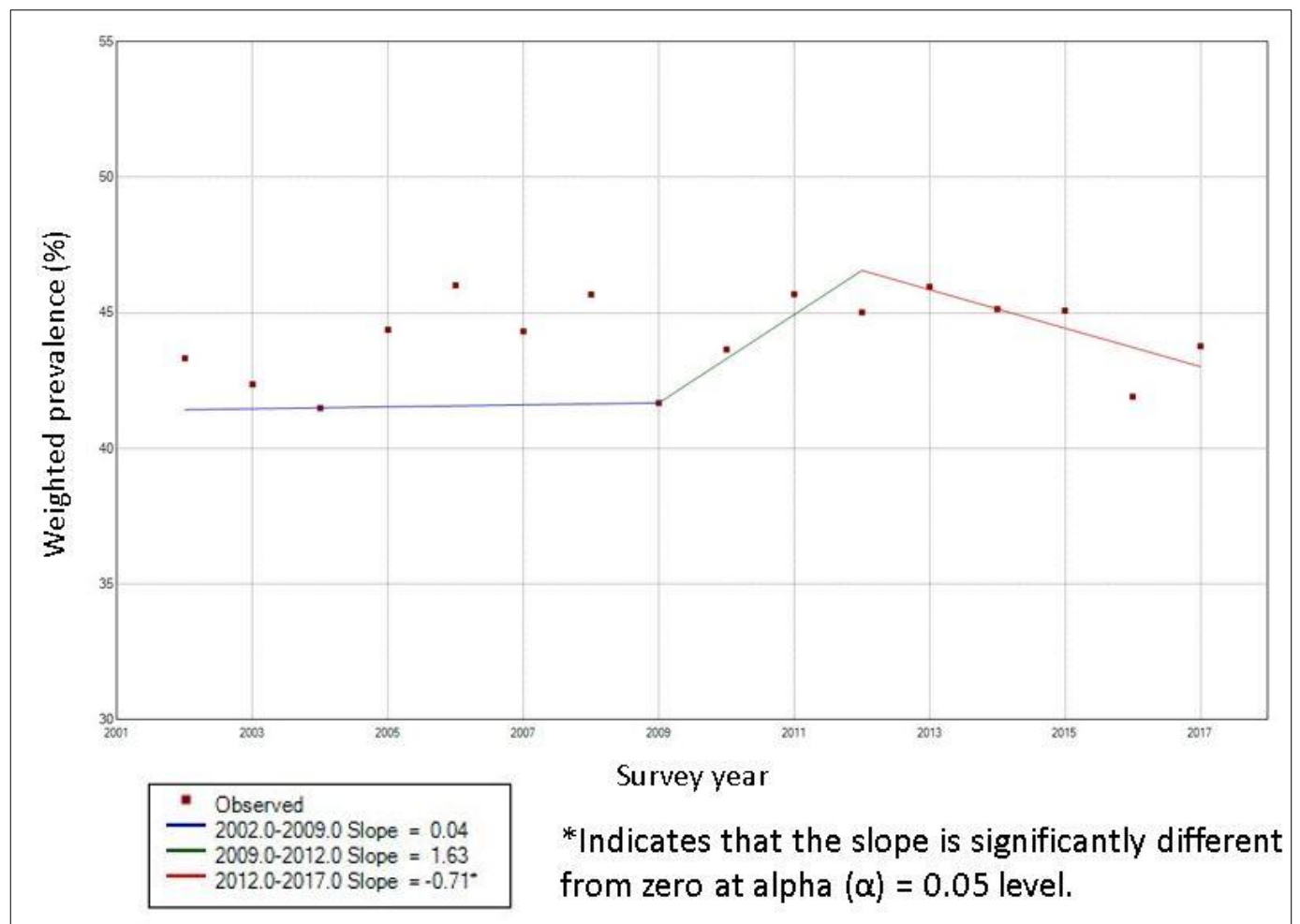

Figure 4.42 $\mid$ Joinpoint analysis of the weighted prevalence of Class $1(n=5,817)$

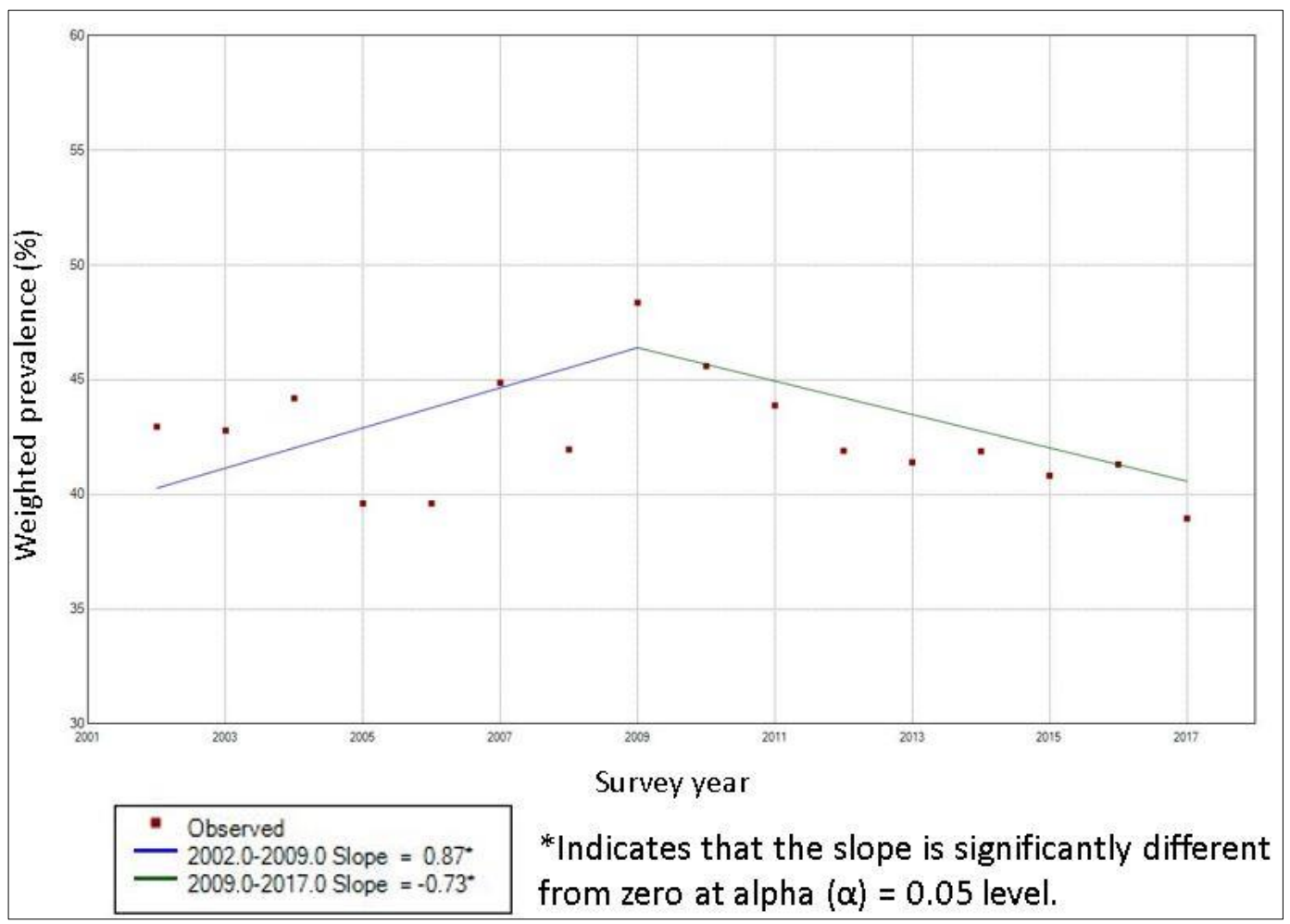

Figure 4.43 $\mid$ Joinpoint analysis of the weighted prevalence of Class $2(n=8,709)$ 


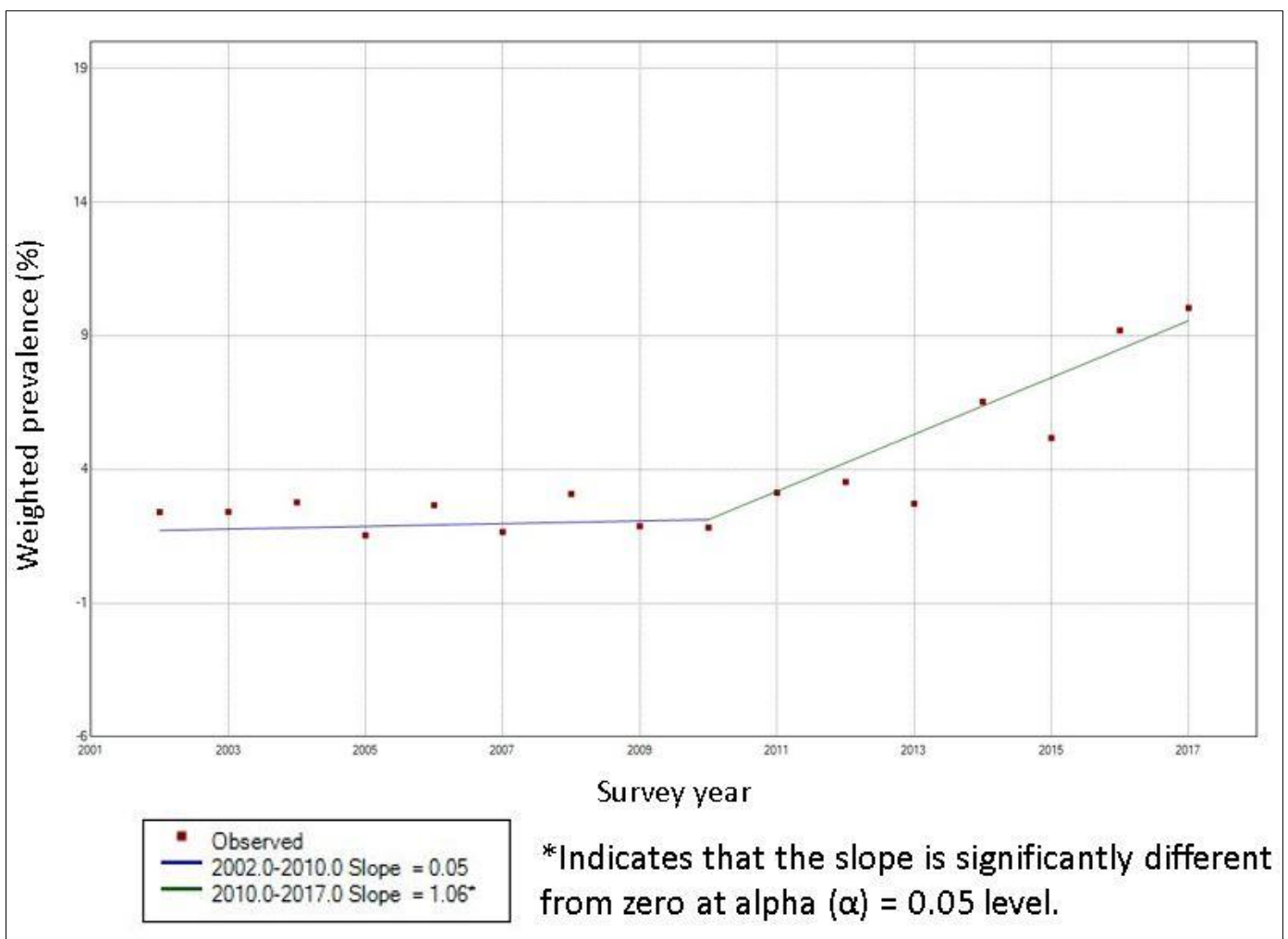

Figure 4.44 | Joinpoint analysis of the weighted prevalence of Class $3(n=553)$

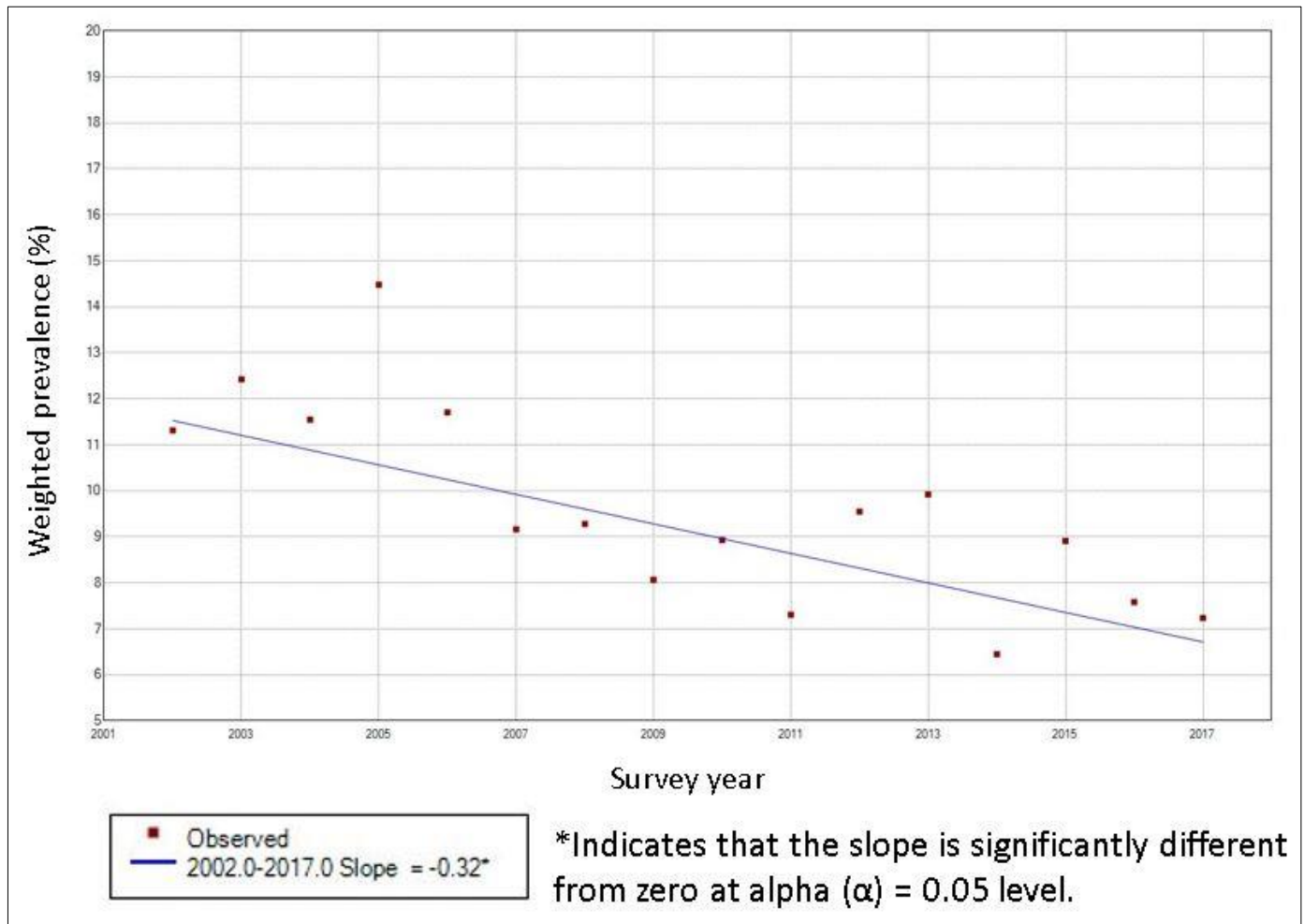

Figure 4.45 $\mid$ Joinpoint analysis of the weighted prevalence of Class $4(n=1,906)$ 


\begin{abstract}
Aim 3 Results
Subaim 3.1: To determine the prevalence and bivariable associations of the following covariates using the latent class variable as an outcome: age, sex, race/ethnicity, total household income, education level, marital status, employment status, health insurance status, age at prescription opioid initiation, opioid use disorder, depression, county type, and residential transience.
\end{abstract}

Before assessing the prevalence and bivariable associations between polydrug latent classes and its correlates, substance use indicator data from 2010 to 2017 was evaluated to determine the best fit number of classes for the subset $(n=7,541)$. (The subset data was used because the goal was to the determine the most current polydrug latent classes starting from the beginning of Wave II of the high opioid mortality). A total of four classes were enumerated using LCA, which were very comparable to those discussed in the results for Aim 2. A description of the four classes were provided below:

- Class 1: PPR - characterized predominantly by PPR and cigarette use, with low endorsement probabilities of other core substances $(n=3,432 ; P R=0.55)$

- Class 2: PPR-heavy alcohol-benzodiazepines-stimulants-marijuana characterized by heroin, heavy alcohol, cigarette, prescription benzodiazepines and stimulants, and marijuana use $(n=3,508 ; P R=0.37)$

- Class 3: Heroin-marijuana - characterized mainly by heroin and marijuana use, with some cocaine and other tobacco product use $(n=341 ; P R=0.042)$ 
- Class 4: Combination opioid-polydrug - characterized by equal probabilities of heroin and PPR use, with higher levels of all other substance use $(n=260$; PR = $0.039)$

(Full results of the fit statistic comparisons, weighted prevalence of the latent classes, and endorsement probabilities for core substance use may be found in ST 4.19 through 4.21 in Appendix A).

After excluding cases with missing data for any correlates used in the model, the total sample size used in the analysis for Aim 3 was 5,103 (67.67\% of the total sample size from 2010 to 2017). (It should be noted that the exclusion of these cases was assumed to be missing at random, judging from the sample size comparisons between Table 4.7 and 4.10). Table 4.10 shows the weighted distributions of the latent classes for the subset data.

Table 4.10 | Overall size and weighted prevalence of the polydrug latent classes among PWUO ( $\mathrm{n}=5,103)$, NSDUH 2010 to 2017

\begin{tabular}{cccccc}
\hline Polydrug latent class & \multirow{2}{*}{$\mathbf{n}$} & \multirow{2}{*}{ SE } & & \multicolumn{2}{c}{ 95\% CI } \\
\cline { 5 - 7 } & & & & LL & UL \\
\hline 1: mainly PPR & 2286 & 55.12 & 0.93 & 51.13 & 59.12 \\
\hline $\begin{array}{c}\text { 2: PPR - heavy alcohol - benzo - } \\
\text { stimulant - marijuana }\end{array}$ & 2436 & 37.00 & 0.61 & 34.37 & 39.63 \\
\hline 3: Heroin - marijuana & 215 & 4.02 & 0.34 & 2.57 & 5.47 \\
\hline 4: Combination opioid - polydrug & 166 & 3.85 & 0.36 & 2.32 & 5.39 \\
\hline
\end{tabular}

Abbreviations: $\mathrm{n}=$ total number of survey respondents; $\%=$ weighted percentage; $\mathrm{SE}=$ standard error; $95 \% \mathrm{CI}=95 \%$ confidence interval; $\mathrm{LL}=$ lower limit; $\mathrm{UL}=$ upper limit

Prevalence of classes characterized predominantly by PPR use represented the majority of PWUO from 2010 to 2017, while groups with high endorsement probabilities of heroin use represented the minority. 
When the relationship between the latent classes and the correlates of interest were assessed, significant associations were found to exist for almost all correlates based on $\chi^{2}$ analysis, with one exception: past year depression. Table 4.11 details the prevalence of each latent class by correlate, while Table 4.12 provides the $\chi^{2}$ test results. 
Table 4.11 $\mid$ Prevalence of set and setting correlates of polydrug latent classes ( $n=5,103)$, NSDUH 2010 to 2017

\begin{tabular}{|c|c|c|c|c|c|c|c|c|c|c|c|c|c|}
\hline \multirow{3}{*}{\multicolumn{2}{|c|}{ Independent variables of interest }} & \multicolumn{12}{|c|}{ Polydrug latent class } \\
\hline & & \multicolumn{3}{|c|}{$\begin{array}{l}\text { Class 1: mainly PPR } \\
\quad(\mathrm{n}=2286)\end{array}$} & \multicolumn{3}{|c|}{$\begin{array}{l}\text { Class 2: PPR - heavy } \\
\text { alcohol - benzo - } \\
\text { stimulant - marijuana } \\
(\mathrm{n}=2436)\end{array}$} & \multicolumn{3}{|c|}{$\begin{array}{l}\text { Class 3: Heroin - } \\
\text { marijuana } \\
(\mathrm{n}=215)\end{array}$} & \multicolumn{3}{|c|}{$\begin{array}{c}\text { Class 4: Combination } \\
\text { opioid - polydrug } \\
(\mathrm{n}=166)\end{array}$} \\
\hline & & $\mathrm{n}$ & $\%$ & SE & $\mathrm{n}$ & $\%$ & SE & $\mathrm{n}$ & $\%$ & SE & $\mathrm{n}$ & $\%$ & SE \\
\hline \multirow[t]{3}{*}{ Age (years) } & 18 to 25 & 1089 & 18.27 & 0.81 & 1703 & 42.97 & 2.97 & 108 & 26.98 & 1.08 & 69 & 20.34 & 2.00 \\
\hline & 26 to 34 & 453 & 24.10 & 0.80 & 448 & 30.91 & 1.45 & 60 & 30.76 & 2.49 & 30 & 25.01 & 9.75 \\
\hline & 35 and older & 744 & 57.62 & 1.58 & 285 & 26.12 & 2.62 & 47 & 42.26 & 3.58 & 67 & 54.65 & 7.84 \\
\hline \multirow[t]{2}{*}{ Sex } & Female & 1258 & 50.07 & 2.16 & 915 & 33.77 & 1.21 & 78 & 34.75 & 2.71 & 67 & 33.87 & 1.59 \\
\hline & Male & 1028 & 49.93 & 2.16 & 1521 & 66.23 & 1.21 & 137 & 65.25 & 2.71 & 99 & 66.13 & 1.59 \\
\hline \multirow[t]{4}{*}{ Race/ethnicity } & NH white & 1442 & 66.31 & 1.07 & 1788 & 77.43 & 2.10 & 152 & 65.60 & 1.86 & 106 & 64.51 & 3.57 \\
\hline & NH black & 298 & 10.60 & 0.20 & 186 & 8.14 & 0.15 & 20 & 14.04 & 4.33 & 22 & 19.86 & 0.86 \\
\hline & Hispanic & 386 & 18.35 & 0.68 & 259 & 9.79 & 1.14 & 31 & 17.14 & 2.73 & 23 & 10.46 & 2.03 \\
\hline & Other NH Groups & 160 & 4.73 & 1.16 & 203 & 4.65 & 0.93 & 12 & 3.22 & 1.84 & 15 & 5.18 & 2.34 \\
\hline $\begin{array}{ll}\text { Total household } & <\$ 30,000\end{array}$ & Id $\quad<\$ 30,000$ & 956 & 33.53 & 0.96 & 1128 & 42.49 & 0.93 & 111 & 52.82 & 2.39 & 91 & 61.45 & 4.70 \\
\hline income $\$$ & $\$ 30,000$ to $\$ 74,999$ & 830 & 37.48 & 1.85 & 865 & 36.14 & 1.41 & 65 & 26.92 & 3.48 & 52 & 25.49 & 3.39 \\
\hline (dollars) & $\$ 75,000$ or more & 500 & 28.99 & 1.12 & 443 & 21.37 & 0.49 & 39 & 20.26 & 3.50 & 23 & 13.06 & 1.48 \\
\hline Education & ess than high school & 432 & 16.57 & 1.01 & 542 & 20.70 & 0.64 & 46 & 21.48 & 2.71 & 50 & 23.39 & 3.57 \\
\hline \multirow[t]{2}{*}{ level } & Iigh school graduate & 751 & 28.62 & 2.31 & 897 & 34.94 & 1.88 & 94 & 37.87 & 0.77 & 69 & 38.56 & 2.08 \\
\hline & At or above college & 1103 & 54.81 & 1.75 & 997 & 44.36 & 1.49 & 75 & 40.65 & 2.05 & 47 & 38.05 & 1.53 \\
\hline
\end{tabular}


(Table 4.11 continued)

\begin{tabular}{|c|c|c|c|c|c|c|c|c|c|c|c|c|c|}
\hline \multirow{3}{*}{\multicolumn{2}{|c|}{$\begin{array}{c}\text { Independent variables of } \\
\text { interest }\end{array}$}} & \multicolumn{12}{|c|}{ Polydrug latent class } \\
\hline & & \multicolumn{3}{|c|}{$\begin{array}{l}\text { Class 1: mainly PPR } \\
\quad(\mathrm{n}=2286)\end{array}$} & \multicolumn{3}{|c|}{$\begin{array}{l}\text { Class 2: PPR - heavy } \\
\text { alcohol - benzo - } \\
\text { stimulant - marijuana } \\
(\mathrm{n}=2436)\end{array}$} & \multicolumn{3}{|c|}{$\begin{array}{l}\text { Class 3: Heroin - } \\
\text { marijuana } \\
(\mathrm{n}=215)\end{array}$} & \multicolumn{3}{|c|}{$\begin{array}{l}\text { Class 4: Combination } \\
\text { opioid - polydrug } \\
(\mathrm{n}=166)\end{array}$} \\
\hline & & $\mathrm{n}$ & $\%$ & SE & $\mathrm{n}$ & $\%$ & SE & $\mathrm{n}$ & $\%$ & SE & $\mathrm{n}$ & $\%$ & SE \\
\hline Employment & Full-time & 1070 & 53.18 & 1.40 & 1128 & 50.00 & 0.49 & 55 & 29.74 & 0.90 & 50 & 30.66 & 2.95 \\
\hline \multirow[t]{2}{*}{ status } & Part-time & 399 & 14.84 & 1.66 & 482 & 17.93 & 0.14 & 38 & 12.64 & 0.50 & 15 & 9.65 & 1.95 \\
\hline & Unemployed & 279 & 8.69 & 1.24 & 410 & 15.04 & 1.12 & 45 & 17.66 & 3.53 & 51 & 28.06 & 5.11 \\
\hline \multicolumn{2}{|c|}{ Other employment status } & 538 & 23.28 & 1.01 & 416 & 17.03 & 1.67 & 77 & 39.96 & 4.68 & 50 & 31.63 & 2.90 \\
\hline \multirow[t]{2}{*}{ Marital status } & Married & 685 & 41.74 & 0.86 & 299 & 19.60 & 1.34 & 23 & 13.04 & 3.12 & 12 & 10.31 & 3.57 \\
\hline & Not married & 1601 & 58.26 & 0.86 & 2137 & 80.40 & 1.34 & 192 & 86.96 & 3.12 & 154 & 89.69 & 3.57 \\
\hline Age at prescription & $0-17$ & 587 & 18.05 & 1.23 & 912 & 30.08 & 0.19 & 44 & 15.15 & 0.78 & 49 & 21.60 & 1.84 \\
\hline \multirow{2}{*}{$\begin{array}{l}\text { opioid initiation } \\
\text { (years) }\end{array}$} & $18-25$ & 691 & 23.42 & 1.23 & 783 & 30.22 & 1.58 & 34 & 11.78 & 0.81 & 24 & 19.56 & 1.40 \\
\hline & 26 and older & 1008 & 58.53 & 0.77 & 741 & 39.71 & 1.77 & 137 & 73.07 & 0.37 & 93 & 58.84 & 2.76 \\
\hline \multirow[t]{2}{*}{ Opioid use disorder } & No & 1817 & 80.53 & 0.56 & 1647 & 67.87 & 0.27 & 41 & 25.09 & 2.33 & 52 & 31.05 & 1.60 \\
\hline & Yes & 469 & 19.47 & 0.56 & 789 & 32.13 & 0.27 & 174 & 74.91 & 2.33 & 114 & 68.95 & 1.60 \\
\hline \multirow[t]{2}{*}{ Depression } & No & 2010 & 87.59 & 0.20 & 2127 & 86.66 & 1.57 & 185 & 84.22 & 2.49 & 136 & 82.97 & 5.53 \\
\hline & Yes & 276 & 12.41 & 0.20 & 309 & 13.34 & 1.57 & 30 & 15.78 & 2.49 & 30 & 17.03 & 5.53 \\
\hline \multirow[t]{2}{*}{ Has health insurance } & No & 56 & 38.94 & 7.01 & 710 & 30.03 & 1.76 & 512 & 21.43 & 1.65 & 62 & 24.91 & 3.28 \\
\hline & Yes & 110 & 61.06 & 7.01 & 1726 & 69.97 & 1.76 & 1774 & 78.57 & 1.65 & 153 & 75.09 & 3.28 \\
\hline \multirow[t]{3}{*}{ County type } & Large metro & 1094 & 59.38 & 0.86 & 1065 & 52.90 & 1.77 & 130 & 74.53 & 2.69 & 97 & 61.22 & 6.69 \\
\hline & Small metro & 833 & 29.15 & 0.29 & 910 & 31.82 & 1.27 & 61 & 20.98 & 3.37 & 52 & 31.94 & 4.81 \\
\hline & Nonmetro & 359 & 11.47 & 0.57 & 461 & 15.28 & 0.82 & 24 & 4.49 & 1.34 & 17 & 6.84 & 1.98 \\
\hline \multirow{3}{*}{$\begin{array}{l}\text { Residential } \\
\text { transience } \\
\text { in past year }\end{array}$} & id not move & 1340 & 67.96 & 0.82 & 1144 & 53.96 & 4.96 & 114 & 56.82 & 7.67 & 71 & 49.51 & 4.09 \\
\hline & Moved once & 511 & 19.58 & 0.61 & 668 & 24.04 & 0.61 & 48 & 22.36 & 9.31 & 39 & 23.77 & 3.78 \\
\hline & ed $2+$ times & 435 & 12.46 & 1.13 & 624 & 22.01 & 4.36 & 53 & 20.82 & 2.00 & 56 & 26.71 & 6.84 \\
\hline
\end{tabular}

Abbreviations: $\mathrm{n}=$ total number of survey respondents; $\%=$ weighted percentage; $\mathrm{SE}=$ standard error 
Table 4.12 $\mid$ Rao-Scott $\chi^{2}$ analyses for the association of latent class membership and set and setting correlates among PWUO ( $\mathrm{n}=5,103)$, NSDUH 2010 to 2017

\begin{tabular}{|c|c|c|c|}
\hline \multicolumn{2}{|c|}{ Independent variables of interest } & \multicolumn{2}{|c|}{ Rao-Scott Analyses } \\
\hline & & $\chi^{2}(\mathrm{df})$ & p-value \\
\hline \multirow{3}{*}{ Age (years) } & 18 to 25 & \multirow{3}{*}{$193.30(6)$} & \multirow{3}{*}{$<0.0001$} \\
\hline & 26 to 34 & & \\
\hline & 35 and older & & \\
\hline \multirow{2}{*}{ Sex } & Female & \multirow{2}{*}{89.48 (3) } & \multirow{2}{*}{$<0.0001$} \\
\hline & Male & & \\
\hline \multirow{4}{*}{ Race/ethnicity } & Non-Hispanic white & \multirow{4}{*}{$63.68(9)$} & \multirow{4}{*}{$<0.0001$} \\
\hline & Non-Hispanic black & & \\
\hline & Hispanic & & \\
\hline & Other Non-Hispanic Groups & & \\
\hline \multirow{3}{*}{$\begin{array}{l}\text { Total household } \\
\text { income (dollars) }\end{array}$} & Less than $\$ 30,000$ & \multirow{3}{*}{$132.80(6)$} & \multirow{3}{*}{$<0.0001$} \\
\hline & $\$ 30,000$ to $\$ 74,999$ & & \\
\hline & $\$ 75,000$ or more & & \\
\hline \multirow{3}{*}{ Education level } & Less than high school & \multirow{3}{*}{$38.76(6)$} & \multirow{3}{*}{$<0.0001$} \\
\hline & High school graduate & & \\
\hline & Currently enrolled or completed college & & \\
\hline \multirow{4}{*}{ Employment status } & Employed full-time & \multirow{4}{*}{$104.04(9)$} & \multirow{4}{*}{$<0.0001$} \\
\hline & Employed part-time & & \\
\hline & Unemployed & & \\
\hline & Other employment status & & \\
\hline \multirow{2}{*}{ Marital status } & Married & \multirow{2}{*}{$209.02(3)$} & \multirow{2}{*}{$<0.0001$} \\
\hline & Not married & & \\
\hline \multirow{3}{*}{$\begin{array}{c}\text { Age at prescription } \\
\text { opioid initiation } \\
\text { (years) }\end{array}$} & $0-17$ & \multirow{3}{*}{$203.06(6)$} & \multirow{3}{*}{$<0.0001$} \\
\hline & $18-25$ & & \\
\hline & 26 and older & & \\
\hline
\end{tabular}

Abbreviations: $\chi^{2}(\mathrm{df})=$ Chi-square statistic (degrees of freedom)

Note: Bolded $\chi^{2}$ and $p$-values denote statistical significance at $\mathrm{p}<0.05$. 
(Table 4.12 continued)

\begin{tabular}{|c|c|c|c|}
\hline \multicolumn{2}{|c|}{ Independent variables of interest } & \multicolumn{2}{|c|}{ Rao-Scott Analyses } \\
\hline & & $\chi^{2}(\mathrm{df})$ & p-value \\
\hline \multirow{2}{*}{ Opioid use disorder } & No & \multirow{2}{*}{$\begin{array}{c}1207.86 \\
(3)\end{array}$} & \multirow{2}{*}{$<0.0001$} \\
\hline & Yes & & \\
\hline \multirow{2}{*}{ Depression } & No & \multirow{2}{*}{$1.77(3)$} & \multirow{2}{*}{0.62} \\
\hline & Yes & & \\
\hline \multirow{2}{*}{ Has health insurance } & Yes & \multirow{2}{*}{$17.86(3)$} & \multirow{2}{*}{$<0.0001$} \\
\hline & No & & \\
\hline \multirow{3}{*}{ County type ${ }^{1}$} & Yes & \multirow{3}{*}{$36.50(6)$} & \multirow{3}{*}{$<0.0001$} \\
\hline & Small metro & & \\
\hline & Nonmetro & & \\
\hline \multirow{3}{*}{$\begin{array}{l}\text { Residential transience } \\
\text { in past year }\end{array}$} & Did not move & \multirow{3}{*}{$28.49(6)$} & \multirow{3}{*}{$<0.0001$} \\
\hline & Moved once & & \\
\hline & Moved two or more times & & \\
\hline
\end{tabular}

Abbreviations: $\chi^{2}(\mathrm{df})=$ Chi-square statistic (degrees of freedom)

Note: Bolded $\chi^{2}$ and $p$-values denote statistical significance at $p<0.05$. 


\section{Subaim 3.2: To determine the multivariable associations of specific combinations of variables using the drug, set, and setting model of substance use behavior to predict membership of polydrug latent classes.}

To understand the full extent of these significant associations using the drug, set, setting framework, a full multinomial logistic regression model was analyzed with all correlates, excluding past year depression. As previously stated, three hypotheses and their related sub-hypotheses were examined using the results of the multinomial logistic regression model. It should be noted that while all results were important to address, only those that were significant were discussed for the remainder of this section due to the number of group comparisons and correlates.

\section{Hypothesis 1: Polydrug latent classes will differ by sociodemographic characteristics.}

Sub-hypothesis 1.1: The differences between the associations will be driven by the types and number of substances used within each Class, with the 'combination opioid polydrug Class' more likely than other Classes to be male, non-Hispanic white, unemployed, younger at time of survey, uninsured, and having less education and less total household income.

The multivariable associations described in the following subsections will address all correlates emphasized in this hypothesis. Table $\mathbf{4 . 1 3}$ shows the associations between sociodemographic factors with latent class membership after adjusting for the other set and setting correlates using Class 1 (mainly PPR) as the referent group. 


\section{Associations with age}

Relative to Class 1 (mainly PPR), Class 2 (PPR-heavy alcohol-benzo-stimulantmarijuana) [aOR (95\% CI): $3.71(2.96,4.66)]$ and Class 3 (heroin- marijuana) [aOR (95\% CI): $2.16(1.49,3.12)]$ were significantly more likely to be younger (i.e., 18 to 25

years of age) than older (i.e., 35 and older) at the time of the survey. Also, Class 2 (PPRheavy alcohol-benzo-stimulant-marijuana) [aOR (95\% CI): $2.32(1.90,2.82)]$ and Class 3 (heroin- marijuana) [aOR $(95 \% \mathrm{CI}): 1.88(1.42,2.50)]$ were more likely to be between the ages of 26 to 34 than 35 years and older relative to Class 1 (mainly PPR).

\section{Associations with sex}

As hypothesized, Class 4 was more likely to be male than female when compared to Class 1 (mainly PPR) [aOR (95\% CI): 2.09 (1.46, 3.00)]. However, Class 2 (PPRheavy alcohol-benzo-stimulant-marijuana) [aOR (95\% CI): 1.97 (1.55, 2.50)] and Class 3 (heroin- marijuana) [aOR (95\% CI): $2.28(1.63,3.20)]$ were also more likely to be male than female relative to Class 1 (mainly PPR).

\section{Associations with race/ethnicity}

Of all group comparisons, Class 2 (PPR-heavy alcohol-benzo-stimulantmarijuana) was the only class to have any significant associations with race/ethnicity when compared to Class 1 (mainly PPR). Compared to non-Hispanic whites, nonHispanic blacks [aOR (95\% CI): 0.56 (0.53, 0.59)], Hispanics [aOR (95\% CI): 0.39 $(0.27,0.57)]$, and other non-Hispanic groups [aOR $(95 \% \mathrm{CI}): 0.68(0.56,0.83)]$ were at 
lower odds of belonging to Class 2 (PPR-heavy alcohol-benzo-stimulant-marijuana) relative to Class 1 (mainly PPR).

\section{Associations with total household income}

Relative to Class 1 (mainly PPR), Class 2 (PPR-heavy alcohol-benzo-stimulantmarijuana) [aOR $(95 \% \mathrm{CI}): 1.30(1.14,1.47)]$, Class 3 (heroin- marijuana) [aOR $(95 \%$ CI): 1.59 (1.10, 2.29)], and Class 4 (combination opioid-polydrug) [aOR (95\% CI): 1.94 $(1.12,3.38)]$ had significantly increased odds of earning a household income that was less than $\$ 30,000$ per year (compared to $\$ 75,000$ or more per year).

\section{Associations with education level}

Although education level was found not to impact the prediction of class membership significantly, the point estimates indicated positive associations between lower education levels (i.e., less than high school or high school graduate status) and membership in Class 2 (PPR-heavy alcohol-benzo-stimulant-marijuana), Class 3 (heroinmarijuana), and Class 4 (combination opioid-polydrug) relative to Class 1 (mainly PPR).

\section{Associations with employment status}

As hypothesized, Class 4 had the highest odds of being unemployed (versus employed) relative to Class 1 (mainly PPR) [aOR (95\% CI): $3.40(3.17,3.65)]$ of all other Classes. This association was also true for Class 3 (heroin- marijuana) when compared to Class 1 (mainly PPR) [aOR (95\% CI): $2.42(1.14,5.31)]$. Other significant associations found between employment status and class membership related to the other 
non-full-time employment categories. Relative to Class 1 (mainly PPR), Class 2 (PPRheavy alcohol-benzo-stimulant-marijuana) [aOR (95\% CI): $1.18(1.13,1.22)]$ and Class 3 [aOR $(95 \% \mathrm{CI}): 1.57(1.38,1.79)]$ were significantly more likely to hold part-time employment than full-time employment. Also, Class 3 (heroin- marijuana) [aOR (95\% CI): $2.51(1.64,3.85)]$ and Class 4 [aOR $(95 \%$ CI $): 1.67(1.51,1.84)]$ had higher odds of holding other types of employment. However, relative to Class 1 (mainly PPR), Class 2 (PPR-heavy alcohol-benzo-stimulant-marijuana) had significantly lower odds of having other types of employment [aOR (95\% CI): $0.82(0.71,0.95)]$.

\section{Associations with marital status}

As hypothesized, Class 4 had the highest odds of being 'not married' relative to Class 1 (mainly PPR) [aOR (95\% CI): $4.13(1.98,8.59)]$ of all other Classes. However, Class 2 (PPR-heavy alcohol-benzo-stimulant-marijuana) [aOR (95\% CI): 1.70 (1.55, 1.88)] and Class 3 (heroin- marijuana) [aOR (95\% CI): $3.25(2.69,3.93)]$ were also more significantly likely not to be married relative to Class 1 (mainly PPR).

\section{Associations with health insurance status}

Although no significant associations were found between each group comparison relative to Class 1 (mainly PPR), the point estimates indicated that all Classes were less likely to have health insurance compared to Class 1 (mainly PPR). Additionally, the adjusted point estimate for Class 4 (combination opioid-polydrug) indicated that members in this Class had the lowest odds of having insurance relative to Class 1 (mainly PPR) [aOR (95\% CI): $0.59(0.24,1.74)]$ 
Table 4.13 Multivariable associations between latent class membership and sociodemographic factors ( $\mathrm{n}=5,103)$, NSDUH 2010 to $2017^{\mathrm{a}, \mathrm{b}}$

\begin{tabular}{|c|c|c|c|c|c|c|c|}
\hline \multirow{2}{*}{\multicolumn{2}{|c|}{ Independent variables of interest }} & \multicolumn{2}{|c|}{$\begin{array}{c}\text { Class } 2 \text { (PPR-heavy } \\
\text { alcohol-benzo-stimulant- } \\
\text { marijuana) vs. Class } 1 \\
\text { (mainly PPR) }\end{array}$} & \multicolumn{2}{|c|}{$\begin{array}{c}\text { Class } 3 \text { (Heroin- } \\
\text { marijuana) vs. Class } 1 \\
\text { (mainly PPR) }\end{array}$} & \multicolumn{2}{|c|}{$\begin{array}{c}\text { Class } 4 \text { (Combination } \\
\text { opioid - polydrug) vs. Class } \\
1 \text { (mainly PPR) }\end{array}$} \\
\hline & & OR $(95 \% \mathrm{CI})$ & p-value & OR $(95 \% \mathrm{CI})$ & p-value & OR $(95 \% \mathrm{CI})$ & p-value \\
\hline \multirow{3}{*}{ Age (years) } & 18 to 25 & $3.71(2.96,4.66)$ & $<0.0001$ & $2.16(1.49,3.12)$ & $<0.0001$ & $0.78(0.58,1.06)$ & 0.12 \\
\hline & 26 to 34 & $2.32(1.90,2.82)$ & $<0.0001$ & $1.88(1.42,2.50)$ & $<0.0001$ & $0.91(0.25,3.40)$ & 0.89 \\
\hline & 35 and older & \multicolumn{6}{|c|}{ referent group } \\
\hline \multirow{2}{*}{ Sex } & Female & \multicolumn{6}{|c|}{ referent group } \\
\hline & Male & $1.97(1.55,2.50)$ & $<0.0001$ & $2.28(1.63,3.20)$ & $<0.0001$ & $2.09(1.46,3.00)$ & $<0.0001$ \\
\hline \multirow{4}{*}{ Race/ethnicity } & Non-Hispanic white & \multicolumn{6}{|c|}{ referent group } \\
\hline & Non-Hispanic black & $0.56(0.53,0.59)$ & $<0.0001$ & $0.93(0.31,2.76)$ & 0.89 & $1.40(0.88,2.23)$ & 0.16 \\
\hline & Hispanic & $0.39(0.27,0.57)$ & $<0.0001$ & $0.91(0.68,1.23)$ & 0.54 & $0.57(0.26,1.26)$ & 0.17 \\
\hline & $\begin{array}{c}\text { Other Non-Hispanic } \\
\text { Groups }\end{array}$ & $0.68(0.56,0.83)$ & 0.0001 & $0.46(0.20,1.03)$ & 0.058 & $0.76(0.13,4.32)$ & 0.54 \\
\hline \multirow{3}{*}{$\begin{array}{c}\text { Total } \\
\text { household } \\
\text { income (USD) }\end{array}$} & Less than $\$ 30,000$ & $1.30(1.14,1.47)$ & $<0.0001$ & $1.59(1.10,2.29)$ & 0.013 & $1.94(1.12,3.38)$ & 0.019 \\
\hline & $\$ 30,000$ to $\$ 74,999$ & $1.05(0.87,1.26)$ & 0.61 & $0.74(0.47,1.18)$ & 0.21 & $0.89(0.74,1.08)$ & 0.26 \\
\hline & $\$ 75,000$ or more & \multicolumn{6}{|c|}{ referent group } \\
\hline
\end{tabular}

Abbreviations: aOR $(95 \% \mathrm{CI})=$ adjusted odds ratio $(95 \%$ confidence interval)

${ }^{\mathrm{a}} \mathrm{All}$ bolded aORs, 95\% CIs, and $\mathrm{p}$-values indicated significance at $\mathrm{p}<0.05$

${ }^{b}$ Model was adjusted for the following set and setting variables: age at prescription opioid initiation, opioid use disorder, health insurance status, county type, and residential transience. 
(Table $4.13^{\mathrm{a}, \mathrm{b}}$ continued)

\begin{tabular}{|c|c|c|c|c|c|c|c|}
\hline \multicolumn{2}{|c|}{ Independent variables of interest } & \multicolumn{2}{|c|}{$\begin{array}{l}\text { Class } 2 \text { (PPR-heavy alcohol- } \\
\text { benzo-stimulant-marijuana) } \\
\text { vs. Class } 1 \text { (mainly PPR) }\end{array}$} & \multicolumn{2}{|c|}{$\begin{array}{l}\text { Class } 3 \text { (Heroin- marijuana) } \\
\text { vs. Class } 1 \text { (mainly PPR) }\end{array}$} & \multicolumn{2}{|c|}{$\begin{array}{c}\text { Class } 4 \text { (Combination opioid } \\
\text { - polydrug) vs. Class } 1 \\
\text { (mainly PPR) }\end{array}$} \\
\hline & & OR $(95 \% \mathrm{CI})$ & p-value & OR $(95 \% \mathrm{CI})$ & $\mathrm{p}$-value & OR $(95 \% \mathrm{CI})$ & p-value \\
\hline \multirow{3}{*}{$\begin{array}{l}\text { Education } \\
\text { level }\end{array}$} & $\begin{array}{l}\text { Less than high } \\
\text { school }\end{array}$ & $1.28(1.00,1.64)$ & 0.05 & $1.05(0.71 .1 .56)$ & 0.80 & $1.08(0.90,1.30)$ & 0.40 \\
\hline & $\begin{array}{l}\text { High school } \\
\text { graduate }\end{array}$ & $1.18(0.89,1.56)$ & 0.24 & $1.33(0.99,1.78)$ & 0.059 & $1.26(0.87,1.83)$ & 0.22 \\
\hline & $\begin{array}{l}\text { Currently enrolled } \\
\text { or completed college }\end{array}$ & \multicolumn{6}{|c|}{ referent group } \\
\hline \multirow{4}{*}{$\begin{array}{l}\text { Employment } \\
\text { status }\end{array}$} & Employed full-time & \multicolumn{6}{|c|}{ referent group } \\
\hline & Employed part-time & $1.18(1.13,1.22)$ & $<0.0001$ & $1.57(1.38,1.79)$ & $<0.0001$ & $1.16(0.84,1.62)$ & 0.37 \\
\hline & Unemployed & $1.24(0.86,1.78)$ & 0.25 & $2.42(1.14,5.13)$ & $\mathbf{0 . 0 2 1}$ & $3.40(3.17,3.65)$ & $<0.0001$ \\
\hline & $\begin{array}{l}\text { Other employment } \\
\text { status }^{1}\end{array}$ & $0.82(0.71,0.95)$ & 0.01 & $2.51(1.64,3.85)$ & $<0.0001$ & $1.67(1.51,1.84)$ & $<0.0001$ \\
\hline \multirow{2}{*}{ Marital status } & Married & \multicolumn{6}{|c|}{ referent group } \\
\hline & Not married & $1.70(1.55,1.88)$ & $<0.0001$ & $3.25(2.69,3.93)$ & $<0.0001$ & $4.13(1.98,8.59)$ & 0.0001 \\
\hline \multirow{2}{*}{$\begin{array}{l}\text { Has health } \\
\text { insurance }\end{array}$} & No & \multicolumn{6}{|c|}{ referent group } \\
\hline & Yes & $0.92(0.67,1.27)$ & 0.61 & $0.95(0.57,1.59)$ & 0.84 & $0.59(0.24,1.47)$ & 0.26 \\
\hline
\end{tabular}

Abbreviations: aOR $(95 \% \mathrm{CI})=$ adjusted odds ratio $(95 \%$ confidence interval)

${ }^{\mathrm{a}} \mathrm{All}$ bolded aORs, $95 \%$ CIs, and $\mathrm{p}$-values indicated significance at $\mathrm{p}<0.05$

${ }^{b}$ Model was adjusted for the following set and setting variables: age at prescription opioid initiation, health insurance status, county type, and residential transience.

${ }^{1}$ Other employment status included: 'has a job or volunteer worker,' 'did not work past week'; 'disabled'; 'keeping house full-time'; 'in school/training'; 'retired'; 'does not have a job, 'some other reason.' 


\section{Hypothesis 2: Polydrug latent classes will differ by their age at prescription opioid initiation and OUD status.}

Sub-hypothesis 2.1: The differences between the associations will be driven by the types and number of substances used within each Class, with the 'combination opioid polydrug Class' more likely than other Classes to have initiated prescription opioid use at an early age and to have opioid use disorder in the past year.

Table 4.14 shows the associations between age at prescription opioid initiation and past year OUD status with latent class membership after adjusting the other set and setting correlates using Class 1 (mainly PPR) as the referent group.

\section{Associations with age at prescription opioid initiation}

The association between Class 2 (PPR-heavy alcohol-benzo-stimulant-marijuana) membership and age at prescription opioid initiation relative to Class 1 (mainly PPR) was not significant. However, Class 3 (heroin- marijuana) [aOR (95\% CI): $0.42(0.30,0.57)$ ] and Class 4 (combination opioid-polydrug) [aOR (95\% CI): $0.81(0.68,0.97)]$ were significantly less likely than Class 1 (mainly PPR) to be less than 17 years of age at the time of prescription opioid initiation. Furthermore, Class 3 (heroin- marijuana) was also significantly less likely to have initiated prescription opioids between the ages of 18 and 25 relative to Class 1 (mainly PPR) [aOR (95\% CI): $0.30(0.28,0.32)]$. 


\section{Associations with opioid use disorder}

Relative to Class 1 (mainly PPR), all other polydrug latent classes were more likely to be classified as having an OUD in the past year, with Class 3 (heroinmarijuana) having the highest odds [aOR $(95 \% \mathrm{CI}): 11.03(6.46,18.82)]$ overall. 
Table 4.14 | Multivariable associations between latent class membership and age at prescription opioid initiation and past year OUD status ( $\mathrm{n}=5,103)$, NSDUH 2010 to $2017^{\mathrm{a}, \mathrm{b}}$

\begin{tabular}{|c|c|c|c|c|c|c|c|}
\hline \multirow{2}{*}{\multicolumn{2}{|c|}{ Independent variables of interest }} & \multicolumn{2}{|c|}{$\begin{array}{c}\text { Class } 2 \text { (PPR-heavy } \\
\text { alcohol-benzo-stimulant- } \\
\text { marijuana) vs. Class } 1 \\
\text { (mainly PPR) }\end{array}$} & \multicolumn{2}{|c|}{$\begin{array}{l}\text { Class } 3 \text { (Heroin- marijuana) } \\
\text { vs. Class } 1 \text { (mainly PPR) }\end{array}$} & \multicolumn{2}{|c|}{$\begin{array}{c}\text { Class } 4 \text { (Combination } \\
\text { opioid - polydrug) vs. Class } \\
1 \text { (mainly PPR) }\end{array}$} \\
\hline & & OR (95\% CI) & $\mathrm{p}$-value & OR $(95 \% \mathrm{CI})$ & $\mathrm{p}$-value & OR $(95 \% \mathrm{CI})$ & $\mathrm{p}$-value \\
\hline \multirow{3}{*}{$\begin{array}{c}\text { Age at prescription } \\
\text { opioid initiation } \\
\text { (years) }\end{array}$} & $0-17$ & $1.22(0.84,1.78)$ & 0.30 & $0.42(0.30,0.57)$ & $<0.0001$ & $0.81(0.68,0.97)$ & 0.021 \\
\hline & $18-25$ & $1.07(0.63,1.82)$ & 0.80 & $0.30(0.28,0.32)$ & $<0.0001$ & $0.72(0.49,1.04)$ & 0.082 \\
\hline & 26 and older & \multicolumn{6}{|c|}{ referent group } \\
\hline \multirow{2}{*}{$\begin{array}{l}\text { Opioid use } \\
\text { disorder }\end{array}$} & No & \multicolumn{6}{|c|}{ referent group } \\
\hline & Yes & $1.60(1.38,1.86)$ & $<0.0001$ & $11.03(6.46,18.82)$ & $<0.0001$ & $7.90(5.50,11.36)$ & $<0.0001$ \\
\hline
\end{tabular}

Abbreviations: aOR $(95 \% \mathrm{CI})=$ adjusted odds ratio $(95 \%$ confidence interval)

${ }^{\mathrm{a}} \mathrm{All}$ bolded aORs, 95\% CIs, and $\mathrm{p}$-values indicated significance at $\mathrm{p}<0.05$

f ${ }^{b}$ Model was adjusted for the following set and setting variables: age, sex, race/ethnicity, total household income, education level, employment status, marital status, health insurance status, county type, and residential transience. 


\section{Hypothesis 3: Polydrug latent classes will differ by their environment.}

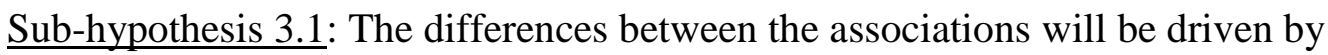
the types and number of substances used within each Class, with the "combination opioid polydrug Class' more likely than other Classes to reside in a large metro and to have moved at least twice in the past year.

Table 4.15 shows the associations between county type and residential transience with latent class membership after adjusting for the other set and setting correlates using Class 1 (mainly PPR) as the referent group.

\section{Associations with county type}

Relative to Class 1 (mainly PPR), Class 3 (heroin- marijuana) and Class 4 (combination opioid-polydrug) were more likely to reside in either a large metro or small metro versus a nonmetro area. Class 3 (heroin- marijuana) was almost five times more likely than Class 1 (mainly PPR) to reside in a large metro [aOR (95\% CI): 4.59 (2.54, 8.32)], and almost three times more likely to live in a small metro area. [aOR (95\% CI): $2.33(1.20,4.52)]$. Also, Class 4 (combination opioid-polydrug) was three times more likely to live in a large metro [aOR $(95 \% \mathrm{CI}): 3.28(1.02,10.56)]$, or to reside in a small metro [aOR $(95 \% \mathrm{CI}): 2.93(1.53,5.62)]$ relative to Class 1 (mainly PPR).

\section{Associations with residential transience in the past year}

While no associations were significant between residential transience and polydrug class membership, point estimates suggested that Class 2 (PPR-heavy alcoholbenzo-stimulant-marijuana) [aOR $(95 \% \mathrm{CI}): 1.33(0.77,2.28)]$ and Class 4 (combination 
opioid-polydrug) [aOR $(95 \% \mathrm{CI}): 1.42(0.93,2.16)]$ were more likely to have moved twice in the past year relative to Class 1 (mainly PPR). 
Table 4.15 | Multivariable associations between latent class membership and county type and residential transience $(\mathrm{n}=5,103)$, NSDUH 2010 to $2017^{\mathrm{a}, \mathrm{b}}$

\begin{tabular}{|c|c|c|c|c|c|c|c|}
\hline \multirow{2}{*}{\multicolumn{2}{|c|}{ Independent variables of interest }} & \multicolumn{2}{|c|}{$\begin{array}{c}\text { Class } 2 \text { (PPR-heavy } \\
\text { alcohol-benzo-stimulant- } \\
\text { marijuana) vs. Class } 1 \\
\text { (mainly PPR) }\end{array}$} & \multicolumn{2}{|c|}{$\begin{array}{c}\text { Class } 3 \text { (Heroin- } \\
\text { marijuana) vs. Class } 1 \\
\text { (mainly PPR) }\end{array}$} & \multicolumn{2}{|c|}{$\begin{array}{c}\text { Class } 4 \text { (Combination opioid } \\
\text { - polydrug) vs. Class } 1 \\
\text { (mainly PPR) }\end{array}$} \\
\hline & & OR $(95 \% \mathrm{CI})$ & $\mathrm{p}$-value & OR $(95 \% \mathrm{CI})$ & p-value & OR $(95 \% \mathrm{CI})$ & $\mathrm{p}$-value \\
\hline \multirow{3}{*}{ County type } & Large metro & $0.97(0.72,1.31)$ & 0.83 & $4.59(2.54,8.32)$ & $<0.0001$ & $3.28(1.02,10.56)$ & 0.047 \\
\hline & Small metro & $0.94(0.77,1.15)$ & 0.54 & $2.33(1.20,4.52)$ & 0.012 & $2.93(1.53,5.62)$ & 0.0012 \\
\hline & Nonmetro & \multicolumn{6}{|c|}{ referent group } \\
\hline \multirow{3}{*}{$\begin{array}{l}\text { Residential } \\
\text { transience in } \\
\text { past year }\end{array}$} & Did not move & \multicolumn{6}{|c|}{ referent group } \\
\hline & Moved once & $1.07(0.69,1.64)$ & 0.77 & $1.00(0.30,3.40)$ & 0.995 & $1.22(0.98,1.53)$ & 0.076 \\
\hline & $\begin{array}{l}\text { Moved two or } \\
\text { more times }\end{array}$ & $1.33(0.77,2.28)$ & 0.31 & $0.92(0.76,1.12)$ & 0.40 & $1.42(0.93,2.16)$ & 0.11 \\
\hline
\end{tabular}

\& Abbreviations: aOR $(95 \% \mathrm{CI})=$ adjusted odds ratio $(95 \%$ confidence interval)

${ }^{\mathrm{a}} \mathrm{All}$ bolded aORs, 95\% CIs, and $\mathrm{p}$-values indicated significance at $\mathrm{p}<0.05$

${ }^{\mathrm{b}}$ Model was adjusted for the following set and setting variables: age, sex, race/ethnicity, total household income, education level, employment status, marital status, health insurance status, age at prescription opioid initiation, and opioid use disorder. 


\section{CHAPTER V}

\section{DISCUSSION}

The current opioid overdose epidemic continues to affect many Americans with uncertainties about how to stop its progression. An aspect that is drawing increased attention is the polydrug use patterns among PWUO, including the trends of co-used substances. Recent articles and CDC reports document an increase in the use of complex combinations multiple drugs in opioid overdose cases. ${ }^{18,35,94,176,204,205}$ Thus, closer attention is needed to track polydrug use patterns among PWUO, trends over time, and risk factors associated with these populations, ${ }^{206}$ to improve policies group-specific interventions targeting this high-risk population. This section provides a concise summary for each Aim in this research, with supporting citations and interpretations for the overall significance of the study. Recommendations based on results from each Aim, as well as final conclusions, were made using the "Cascade of Care" framework. ${ }^{207}$

\section{Longitudinal Prevalence of People Who Use Opioids and Their Substance Use Patterns}

The analyses presented in this dissertation document the decrease over time from 2002 to 2017 in the prevalence of all PWUO. When stratified into the three opioid use groups, it is clear the overall proportion of past month PPR-only use group far exceeded 
that of the heroin-only and combination opioid groups, however the prevalence of past month heroin-only and combination opioid use groups increased over time, while the past month PPR-only use group steadily decreased from 2002 to 2017 . The decrease in the prevalence of the latter group may support the possible success of certain federal and state policies (e.g., Prescription Drug Abuse Prevention Plan, prescription drug monitoring programs - PDMPs). ${ }^{208,209}$ However, another macro-level factor that could have contributed to these opposing trends is the reformulation of OxyContin, which may have led to the initiation of heroin use among those who only use PPR, ${ }^{210}$ explaining the increases in the prevalence of the past month heroin-only and combination opioid use. Because it has been shown that individuals that co-use PPR and heroin experience a high burden of polydrug use, the second step in assessing overdose risk among PWUO should be to track the patterns of reported polydrug use among the three opioid use groups. ${ }^{21}$

In the past month heroin-only use group, other core substances which were reported by at least $20 \%$ of the subgroup were cocaine, binge alcohol, other tobacco products, and crack use. In the past month combination opioid use group, all substances except for stimulants and hallucinogen/inhalants were reported by at least $20 \%$ of the subgroup. These results indicate a progressively larger number of substances being used in these two subgroups, especially illicit substances such as cocaine and crack. These results align with previous research where mortality rates related to cocaine and opioid use were shown to be increasing after $2010 .{ }^{18}$

In terms of substance use trajectories, the only core substance whose prevalence significantly increased during the later years among all PWUO was past month prescription stimulant use. For the past month PPR-only use group, only the past month 
use of other tobacco products significantly increased in prevalence, with a simultaneous significant decrease in the trend of cigarette use. While it is encouraging to see the decrease in cigarette use, this remains a concern because it has been shown that smokeless tobacco is not a safer alternative to cigarettes. ${ }^{211}$ Products such as snuff, chewing tobacco, and even nicotine gum remain in the circulatory system at higher concentrations for longer amounts of time. ${ }^{212}$ Therefore, chronic pain patients who use smokeless tobacco products as an option to alleviate their pain symptoms may experience a prolonged sense of pain relief, which could exacerbate addictive behaviors. Finally, among the past month combination opioid use group, the core substances whose prevalence significantly increased over time were crack and prescription stimulants. As evident from the results of the prevalence and trends of reported substance use behavior among the three opioid use groups, patterns of use appeared to be dependent on the opioid use group. In general, those belonging to the past month heroin-only and combination opioid groups had a higher prevalence of reporting use of core substances. The number of core substances that were reported to have been in use by more than $20 \%$ of past month combination opioid users included illicit substances (i.e., cocaine and crack), which is of concern.

The results from Aim 1 lead to the following policy recommendations. Greater attention needs to be directed towards detecting and tracking changes in patterns of polydrug use among PWUO. Clinicians and drug dispensaries need to be more cognizant of a patient's prescription history, especially given the continued rise of the co-use of opioids and prescription stimulants. To achieve this goal, health management leadership and policymakers may promote the utility of a nationwide Universal Electronic Health 
Records system, improve the interoperability of data from the prescription drug monitoring programs (PDMP) to be used among all States, and even the integration of both platforms to improve tracking efforts of prior substance use history. ${ }^{213,214}$ The merging of electronic health record and PDMP systems could resolve issues that may physicians have reported in regards to the difficulty of use, which has been shown as being an ineffective method to reduce the practice of prescribing opioids. ${ }^{215}$ Additionally, tobacco use cessation programs could be leveraged to promote long-term abstinence among those who have sought treatment for an OUD. ${ }^{216}$ Finally, more formal policies on the use of medications for cocaine withdrawal and abstinence initiation (e.g., propranolol) and relapse prevention (e.g., baclofen) could be put into standard use in emergency rooms nationwide for patients presenting with positive screens for cocaine and other opioids. ${ }^{217}$

\section{Polydrug Latent Class Composition and Trends}

Four different polydrug use patterns were identified for this study using latent class analysis, which captured one dimension of use: type of substance. Class 1 was characterized by the past month use of mainly PPR and low levels of all other core substances and represented the highest probabilities of all opioid-related polydrug use groups [PR (SE): $0.55(0.93)]$. Class 2 was characterized by high probabilities of heroin, heavy alcohol, cigarette, prescription tranquilizers/sedatives (i.e., benzodiazepines), prescription stimulants, and marijuana use, and was the second largest opioid-related polydrug latent class [PR (SE): $0.37(0.61)$ ]. Class 3 was largely characterized by heroin and marijuana use, followed by some cocaine and other tobacco use, and represented 
almost four percent of all the opioid-related polydrug latent classes [PR (SE): 0.0361 (0.16)]. Finally, Class 4 was characterized by prescription pain reliever and heroin use, with a high prevalence of all other core substances [PR (SE): 0.096 (0.34)].

This study indicates that, while prevalence trends for Class 1 (mainly PPR), Class 2 (PPR - heavy alcohol-benzo-stimulant-marijuana), and Class 4 (combination opioidpolydrug) has decreased since 2010, the prevalence of Class 3 (heroin-marijuana-illicit substances) has increased.

The results of Aim 2 for the LCA composition and time trends in this study clarified and reinforced those of Aim 1. The pattern identified as Class 3 (heroinmarijuana-illicit substance users) showed a significant, recent doubling in prevalence during the period 2014-2017. This suggests that the prevalence of populations with riskier substance use behaviors (i.e., Class 3 ) is increasing over time, and may possibly be drawn from lower risk populations such as Class 1 (mainly PPR), given their diminishing prevalence. Therefore, one policy recommendation based on the results of Aim 2 would be to establish a routine surveillance program to promote pharmacovigilance among PWUO. ${ }^{218}$ This may require yearly or semi-yearly analyses of polydrug use behavior which draws from surveys such as the NSDUH, or online platforms (e.g., Twitter, Instagram, Reddit, etc.). ${ }^{218}$ Other avenues that could be pursued would necessitate the involve pharmaceutical companies to conduct their own pharmacoepidemiologic studies as a means of post-marketing surveillance in order to inform public health authorities, especially given their expansive resources. Finally, because the abuse of and dependence on substances are considered mental health disorders, alternative therapies beyond medicinal approaches for chronic pain should be standardized in opioid use treatment. 
One type of alternative therapy that has been shown in recent years to aid in opioid use outcomes is cognitive behavioral therapy (i.e., psychotherapy), with some evidence of its efficacy without the use of an additional MAT (e.g., buprenorphine). ${ }^{219,220}$

\section{Polydrug Latent Classes During Wave II of High Opioid Mortality and Associations with Set and Setting Correlates}

As previously discussed, Wave II of high opioid mortality in the U.S. was due to

the use of heroin and was said to begin in $2010 .{ }^{76}$ Because this was arguably the start of the changing risk environment of opioid overdose risk, it was decided that a polydrug latent class would be modeled using data from 2010 to 2017 . This provided a more current assessment of the associations with the set and setting correlates during a time of riskier polydrug use.

As expected, most correlates used in this study were significantly associated with membership in the polydrug latent classes, except for past year depression when using $\chi^{2}$ testing. Results of the multinomial logistic regression model clarified these significant associations but in a manner that was not expected. It was hypothesized that Class 4 (combination opioid - polydrug) would be more likely:

1. To be male, non-Hispanic white, unemployed, unmarried, younger at the time of the survey, uninsured, and having less education and less total household income. (Set characteristics)

2. To have initiated prescription opioid use at an earlier age and to have past year OUD. (Set characteristics) 
3. To be more likely to reside in a large metropolitan area and to have moved at least twice in the past year. (Setting characteristics)

These sub-hypotheses were formed based on findings from past research on multiple substance use, particularly those studies that have determined that subpopulations of PWUO who exhibit less polydrug use behavior are less likely to be characterized with higher overdose risk behaviors. ${ }^{19,36,37}$ Although the substance use indicators in this study do not necessarily inform on the biological effects of polydrug use (i.e., null, overlapping, additive, or antagonistic effects), ${ }^{221}$ it stands to reason that a higher number of possible substance use combinations, especially within a relatively short amount of time, would result in a higher likelihood of overdose.

Overall, the hypothesized relationship between Class 4 (combination opioid polydrug) and all of the set and setting correlates were supported the hypothesized pattern; however, Class 3 was shown to have a stronger association for several correlates, Not only did Class 4 (combination opioid - polydrug) have the greatest odds of being unemployed and not being married, members of this class were more likely to reside in a small metropolitan area. However, compared to Classes 2 and 4, members of Class 3 (heroin-marijuana), had the highest odds of OUD [aOR (95\% CI): $11.03(6.46,18.82)]$ relative to Class 1 (mainly PPR). Additionally, relative to Class 1, Class 3 was least likely to have initiated prescription opioid use at a younger age, which suggests that substance use experience during adolescence did not greatly affect whether Classes that exhibit greater polydrug use behaviors eventually develop an OUD. Additionally, while employment status varied for Class 2, 3, and 4 relative to Class 1, overall results indicate that these three Classes had higher odds of not being employed fulltime, which may have 
implications with increased access to health insurance and other work-related benefits. ${ }^{163-}$ 165

These results lead to the following inferences and recommendations. Although health insurance status was not significantly associated with predicting polydrug latent class membership, lower income level was shown to be more likely among Class 2 (PPRheavy alcohol-benzo-stimulant-marijuana) and Class 4 (combination opioid-polydrug) groups. This supports the idea that affordable substance abuse treatment services might be made more available, especially in small and large metropolitan areas. Substance use services within any major locale should involve the local community, local government, and State government support (via Medicaid expansion). If executed properly, expanded access at a reasonable cost for services like medication-assisted treatment (MAT) and even substance use counseling (e.g., cognitive behavioral therapy) could be provided to those who are suffering from substance use disorders. Finally, community programs that provide counseling and therapeutic environments (e.g., The Living Room) $)^{222}$ may provide an additional outlet of mental and physical support for those suffering from substance use disorders.

\section{Strengths and Limitations}

The primary strengths of this study were the use of a large, national database consisting of repeated surveys of drug use over a 16-year period (NSDUH) and the application of statistical methods for tracking changes in patterns of use over this period (LCA and joinpoint regression). The research was also based on a strong theoretical model with policy and prevention recommendations. Trends in the reported polydrug use 
among PWUO were documented and analyzed using joinpoint regression, which allows direct tests of whether trends are statistically significant, as well as identifying specific times where trends may shift. These analyses provided a clearer identification of both when and how patterns of polydrug use changed in PWUO. Also, the person-centered LCA approach used uncovered and clarified subpopulations of PWUO polydrug patterns and allowed a better assessment of their associations with demographic and behavioral correlates within the $d r u g$, set, and setting framework. Thus, this research adds meaningfully to the literature in this area by providing a more detailed assessment of both patterns of polydrug use and changes over time. Other strengths inherent in the NSDUH include a large number of yearly participants resulting in precise prevalence estimates.

There are several limitations of this study, mostly related to the survey methodology. First, the data collected in the NSDUH relies on the self-report of participants, which may affect final results in the study because of different types of information bias, including (but not limited to) recall bias and non-response or participation bias, especially given the sensitive nature of the survey questions. While all efforts were made to promote truthfulness in answering sensitive survey questions, the likelihood of this may be questioned when participants are asked about illicit substance use. However, participants in NSDUH were allowed to complete an audio computerassisted self-interview (ACASI) for all substance use and other sensitive questions which may have helped overcome some of this potential information bias. With regard to participation bias, individuals who were homeless, served in the military, or were incarcerated were excluded because they do not have fixed home addresses. ${ }^{223}$ The exclusion of these populations is problematic because it has been suggested that the 
prevalence of opioid use is systematically undercounted. ${ }^{224}$ However, it is difficult to determine whether this undercounting necessarily biased findings with regard to latent class patterns of use or time trends. This problem might be addressed, given appropriate data, through similar analyses of other community-based samples, such as community centers focused on substance use rehabilitation or mental health treatment, publicly managed halfway homes for recent parolees, or patients at the Veteran's Administration $(\mathrm{VA}) .^{225-227}$

Second, NSDUH is a cross-sectional survey, which technically does not allow longitudinal analyses of changes within persons. Nonetheless, repeated surveys within a well-defined target sample may be used to describe changes in a population over time. Third, the survey methodology from 2002 to 2017 has changed at various stages making results from some years incomparable to others, particularly when the change is related to the wording of a survey question. Also, the inclusion of illicit methamphetamine use could not be included in the analyses because that module was not available until 2015.

Lastly, this study utilized the publicly available data from the 2002 through 2017 cycles of NSDUH, statewide variations in substance use behavior were not be assessed, and some sociodemographic variables could not be included in characterizing latent polydrug groups among PWUO. Also, due to the design of the survey questions, simultaneous and concurrent use could not be distinguished in this study, which hinders any specific interpretation about co-use behaviors.

Some limitations may also be viewed from an analytical standpoint. Nonsignificant associations between Class 3 (heroin-marijuana) or Class 4 (combination opioid - polydrug) membership with some of the set and setting correlates may have been 
a result of small sample size $(n=341)$, which may have increased the likelihood of making a Type II error. Also, while the total sample size was large after the exclusion of all individuals who reported not having used heroin or PPR in the past month, examination of the sample size by each opioid use group across 16 years necessitated the collapse of certain categories, including time, race, education, income, marital status, OUD status, etc. Finally, this study assumed that the two latent classes enumerated for Aims 2 and 3 are the same or closely similar and that any time-dependent differences will be reflected in the associations of the set and setting covariates.

And because the latent class analysis was the focus of this study, several limitations arise from this complex modeling technique. First, deciding the number of classes in a latent variable has been a topic of discussion among many social science statisticians, with varying guidelines to assist in the determination of the total number of classes. $^{202,228,229}$ Second, while power may be inferred using simulation studies from external researchers, this is limited only to the ability of a fit statistic in aiding in the determination of the final number of classes. Power was not considered once the latent class variable is used in inferential testing because there is no true prevalence that could be used in determining sample size and power formulas, given the probabilistic nature of modeling a latent variable. Finally, although no formal discussion has been found on the co-use of Mplus and SAS for analysis, it may be likely that robustness of point estimates and standard errors may have been affected.

\section{Future Directions}


Simultaneous use of these core substances among PWUO should be incorporated into the NSDUH. Understanding polydrug use with a simultaneous and concurrent distinction would provide more information on the prospective effects of polydrug use and set a rudimentary basis upon which to prompt clinical or animal trials for the pharmacological interactions of multiple substance use. Additionally, because illicitly manufactured fentanyl (IMF) was not able to be assessed within the context of this study, it may be advisable for a separate module related to IMF use to be added into future versions of the NSDUH. Questions for this module could help to sort out an individual's history into using fentanyl; whether this use was intentional or unintentional; and reasons for intentional use. Moreover, the prevalence of intentional IMF use may be assessed in order to further advocate for higher level measures of overdose prevention strategies, including the use of mass distribution of fentanyl test strips. ${ }^{230}$

\section{Conclusions}

The trends of the reported substance use among each of the opioid use groups demonstrate the growing need for expanded programs that recognize the growing trend towards polydrug use in the provision of substance use treatment and rehabilitation. The results of this study also may provide public health practitioners with better substance use information that may be used to develop screening programs for problematic, polydrug use, with the hope of stemming the progression of increased polydrug use especially among those with have only reported the past month use of PPRs.

The present results, including trends, suggest that the polydrug latent class that requires the most attention is Class 3 (heroin-marijuana). This recommendation is guided 
mainly by the increased odds of having OUD in the past year relative to all other classes, even after adjusting for the set and setting risk factors. While the other characteristics of Class 3 do not necessarily conform to the sociodemographic and environmental factors that have been attributed to higher overdose risk, Class 3 (heroin-marijuana) has been significantly increasing since 2010 , so its identification and further study is warranted. However, prevention must begin at the level of lower polydrug use populations among PWUO (e.g., Class 1).

Policy recommendations based on the results of this study fall into three broad categories: clinical interventions directed at individuals, population-level interventions which seek to address polydrug surveillance, and community-level social support and treatment. These recommendations were formed using the "Cascade of Care Framework" for the opioid epidemic. ${ }^{231}$ The Cascade of Care Framework may be summarized as follows: ${ }^{231}$ prevention, identification, treatment, and recovery.

Prevention initiatives may be in the form of using universal electronic health records to prevent an individual from possibly receiving excessive prescription pain relievers in an ED or urgent care facility, especially if integrated with prescription drug monitoring program systems. Identifying individuals at high risk of developing OUD should include higher level pharmacovigilance efforts by local, state, and federal governments or pharmaceutical companies. These efforts may necessitate using data from the NSDUH or following social platforms online. Treatment should include medication-assisted treatments, but also holistic approaches (e.g., cognitive behavioral therapy), or community-level substance use and mental health initiatives, in order to increase the success of long-term recovery efforts. 
In summary, the research conducted in the present study provides a model for improved understanding of the growing problem of polydrug use within PWUO, changes over time in patterns of polydrug use, and identification of those at greatest risk, that may lead to stronger policies, screening practices, and treatments to prevent drug overdose outcomes. 


\section{REFERENCES}

1. Acker CJ, Tracy SW. Altering American consciousness: the history of alcohol and drug use in the United States, 1800-2000. Amherst, Mass.: University of Massachusetts Press; 2004.

2. Davenport-Hines RPT. The pursuit of oblivion : a global history of narcotics. 1st American ed. New York: Norton; 2002.

3. McNulty S. From deity to demon: the social implications of opiate addiction in late nineteenth and early twentieth century America. New Brunswick, NJ:

Department of History, Rutgers University; 2009.

4. deShazo RD, Johnson M, Eriator I, Rodenmeyer K. Backstories on the US Opioid Epidemic. Good Intentions Gone Bad, an Industry Gone Rogue, and Watch Dogs Gone to Sleep. Am J Med. 2018;131(6):595-601.

5. Kolodny A, Courtwright DT, Hwang CS, et al. The prescription opioid and heroin crisis: a public health approach to an epidemic of addiction. Annu Rev Public Health. 2015;36:559-574.

6. Portenoy RK, Foley KM. Chronic use of opioid analgesics in non-malignant pain: report of 38 cases. Pain. 1986;25(2):171-186.

7. Rudd RA, Seth P, David F, Scholl L. Increases in Drug and Opioid-Involved Overdose Deaths - United States, 2010-2015. MMWR Morb Mortal Wkly Rep. 2016;65(5051):1445-1452.

8. Hedegaard H, Warner M, Minino AM. Drug Overdose Deaths in the United States, 1999-2016. NCHS Data Brief. 2017(294):1-8.

9. Centers for Disease Control and Prevention. Understanding the epidemic. 2016; https://www.cdc.gov/drugoverdose/epidemic/index.html. Accessed 27 June 2017.

10. Warner M, Trinidad JP, Bastian BA, Minino AM, Hedegaard H. Drugs Most Frequently Involved in Drug Overdose Deaths: United States, 2010-2014. Natl Vital Stat Rep. 2016;65(10):1-15.

11. Frank RG, Pollack HA. Addressing the Fentanyl Threat to Public Health. $N$ Engl J Med. 2017;376(7):605-607. 
12. National Institute on Drug Abuse. Trends \& Statistics. 2017; https://www.drugabuse.gov/related-topics/trends-statistics. Accessed December $10,2017$.

13. Birnbaum HG, White AG, Schiller M, Waldman T, Cleveland JM, Roland CL. Societal costs of prescription opioid abuse, dependence, and misuse in the United States. Pain Med. 2011;12(4):657-667.

14. Florence CS, Zhou C, Luo F, Xu L. The Economic Burden of Prescription Opioid Overdose, Abuse, and Dependence in the United States, 2013. Med Care. 2016;54(10):901-906.

15. Dart RC, Surratt HL, Cicero TJ, et al. Trends in opioid analgesic abuse and mortality in the United States. N Engl J Med. 2015;372(3):241-248.16. Cicero TJ, Ellis MS, Harney J. Shifting Patterns of Prescription Opioid and Heroin Abuse in the United States. N Engl J Med. 2015;373(18):1789-1790.

17. McCall Jones C, Baldwin GT, Compton WM. Recent Increases in CocaineRelated Overdose Deaths and the Role of Opioids. American Journal of Public Health. 2017;107(3):430-432.

18. Jones CM, Logan J, Gladden RM, Bohm MK. Vital Signs: Demographic and Substance Use Trends Among Heroin Users - United States, 2002-2013. MMWR Morb Mortal Wkly Rep. 2015;64(26):719-725.

19. Wu LT, Woody GE, Yang C, Blazer DG. Subtypes of nonmedical opioid users: results from the national epidemiologic survey on alcohol and related conditions. Drug Alcohol Depend. 2010;112(1-2):69-80.

20. Wunsch MJ, Nakamoto K, Behonick G, Massello W. Opioid deaths in rural Virginia: a description of the high prevalence of accidental fatalities involving prescribed medications. The American journal on addictions. 2009;18(1):5-14.

21. Mital S, Windle M, Cooper HLF, Crawford ND. Trends in non-medical prescription opioids and heroin co-use among adults, 2003-2014. Addictive Behaviors. 2018;86:17-23.

22. Elinson J, Nurco D. Operational definitions in socio-behavioral drug use research, 1975. Rockville, Md.: National Institute on Drug Abuse; 1975.

23. Martin CS, Clifford PR, Clapper RL. Patterns and predictors of simultaneous and concurrent use of alcohol, tobacco, marijuana, and hallucinogens in first-year college students. J Subst Abuse. 1992;4(3):319-326.

24. Earleywine M, Newcomb MD. Concurrent versus simultaneous polydrug use: prevalence, correlates, discriminant validity, and prospective effects on health outcomes. Exp Clin Psychopharmacol. 1997;5(4):353-364. 
25. McClure FL, Niles JK, Kaufman HW, Gudin J. Concurrent Use of Opioids and Benzodiazepines: Evaluation of Prescription Drug Monitoring by a United States Laboratory. J Addict Med. 2017;11(6):420-426.

26. Dasgupta N, Funk MJ, Proescholdbell S, Hirsch A, Ribisl KM, Marshall S. Cohort Study of the Impact of High-Dose Opioid Analgesics on Overdose Mortality. Pain Med. 2016;17(1):85-98.

27. McCabe SE, Cranford JA, Boyd CJ. The relationship between past-year drinking behaviors and nonmedical use of prescription drugs: prevalence of co-occurrence in a national sample. Drug Alcohol Depend. 2006;84(3):281-288.

28. Wu LT, Blazer DG, Li TK, Woody GE. Treatment use and barriers among adolescents with prescription opioid use disorders. Addict Behav. 2011;36(12):1233-1239.

29. Brecht ML, Huang D, Evans E, Hser YI. Polydrug use and implications for longitudinal research: ten-year trajectories for heroin, cocaine, and methamphetamine users. Drug Alcohol Depend. 2008;96(3):193-201.

30. Ives TJ, Chelminski PR, Hammett-Stabler CA, et al. Predictors of opioid misuse in patients with chronic pain: a prospective cohort study. BMC Health Services Research. 2006;6:46-46.

31. Baggio Sp, Studer J, Mohler-Kuo M, Daeppen J-B, Gmel G. Concurrent and simultaneous polydrug use among young Swiss males: use patterns and associations of number of substances used with health issues. info:doi/101515/ijamh-2013-0305. 2013. http://www.zora.uzh.ch/id/eprint/88522/1/ZORA88522.pdf.

32. Tibbits MK, Smith EA, Caldwell LL, Flisher AJ. Impact of HealthWise South Africa on polydrug use and high-risk sexual behavior. Health Education Research. 2011;26(4):653-663.

33. Meacham MC, Roesch SC, Strathdee SA, Lindsay S, Gonzalez-Zuniga P, Gaines TL. Latent classes of polydrug and polyroute use and associations with human immunodeficiency virus risk behaviours and overdose among people who inject drugs in Tijuana, Baja California, Mexico. Drug and alcohol review. 2018;37(1):128-136.

34. Meacham MC, Rudolph AE, Strathdee SA, et al. Polydrug Use and HIV Risk Among People Who Inject Heroin in Tijuana, Mexico: A Latent Class Analysis. Subst Use Misuse. 2015;50(10):1351-1359.

35. Lorvick J, Browne EN, Lambdin BH, Comfort M. Polydrug use patterns, risk behavior and unmet healthcare need in a community-based sample of women who use cocaine, heroin or methamphetamine. Addictive Behaviors. 2018;85:94-99. 
36. Roth AM, Armenta RA, Wagner KD, et al. Patterns of drug use, risky behavior, and health status among persons who inject drugs living in San Diego, California: a latent class analysis. Subst Use Misuse. 2015;50(2):205-214.

37. Bobashev G, Tebbe K, Peiper N, Hoffer L. Polydrug use among heroin users in Cleveland, OH. Drug Alcohol Depend. 2018;192:80-87.

38. Kalyanam J, Katsuki T, G RGL, Mackey TK. Exploring trends of nonmedical use of prescription drugs and polydrug abuse in the Twittersphere using unsupervised machine learning. Addict Behav. 2017;65:289-295.

39. John WS, Wu LT. Trends and correlates of cocaine use and cocaine use disorder in the United States from 2011 to 2015. Drug Alcohol Depend. 2017;180:376384.

40. Center for Behavioral Health Statistics and Quality. 2017 National Survey on Drug Use and Health Final Analytic File Codebook. In. Rockville, MD: Substance Abuse and Mental Health Services Administration; 2018.

41. Bourne PG. Polydrug abuse--considerations in a national strategy. Am J Drug Alcohol Abuse. 1974;1(2):147-158.

42. Wesson DR, Smith DE, Lerner SE, Kettner VR. Treatment of polydrug users in San Francisco. Am J Drug Alcohol Abuse. 1974;1(2):159-179.

43. Kamali K, Steer RA. Polydrug use by high-school students: involvement and correlates. Int J Addict. 1976;11(2):337-343.

44. Ramer CM, Lodge A. Neonatal addiction: a two-year study. Part I. Clinical and developmental characteristics of infants of mothers on methadone maintenance. Addict Dis. 1975;2(1-2):227-234.

45. Curtis B, Simpson DD. Demographic characteristics of groups classified by patterns of multiple drug abuse: a 1969-1971 sample. Int J Addict. 1976;11(1):161-173.

46. Nixon R. Special Message to the Congress on Drug Abuse Prevention and Control. 1971; http://www.presidency.ucsb.edu/ws/?pid=3048.

47. Caputi CA, Busca G, Fogliardi F, Giugliano F. Evaluation of tolerance in longterm treatment of cancer pain with epidural morphine. Int J Clin Pharmacol Ther Toxicol. 1983;21(11):587-590.

48. Lindgren U, Djupsjo H. Diclofenac for pain after hip surgery. Acta Orthop Scand. 1985;56(1):28-31.

49. Black RG. The chronic pain syndrome. Surg Clin North Am. 1975;55(4):9991011. 
50. Maruta T, Swanson DW, Finlayson RE. Drug abuse and dependency in patients with chronic pain. Mayo Clin Proc. 1979;54(4):241-244.

51. Turner J, Calsyn D, Fordyce W, Ready L. Drug utilization patterns in chronic pain patients. Pain. 1982;12(4):357-363.

52. Porter J, Jick H. Addiction Rare in Patients Treated with Narcotics. New England Journal of Medicine. 1980;302(2):123-123.

53. Pasternak SR. Personality Traits and Cognitive Style in Chronic Pain Patients. In. New York, NY: Columbia University; 1980:181.

54. Taub. Opioid analgesics in the treatment of chronic intractable pain of nonneoplastic origin. Pain. 1984;18.

55. Tennant FS, Uelmen GF. Narcotic maintenance for chronic pain. Medical and legal guidelines. Postgraduate medicine. 1983;73(1):81-83.

56. France RD, Urban BJ, Keefe FJ. Long-term use of narcotic analgesics in chronic pain. Social Science \& Medicine. 1984;19(12):1379-1382.

57. Phillips R, Herning R, London E. Morphine effects on the spontaneous electroencephalogram in polydrug abusers: correlations with subjective selfreports. Neuropsychopharmacology. 1994;10(3):171-181.

58. Lichtigfeld FJ, Gillman MA. Combination therapy with carbamazepine/benzodiazepine for polydrug analgesic/depressant withdrawal. $J$ Subst Abuse Treat. 1991;8(4):293-295.

59. Batts K, Pemberton M, Bose J, et al. Comparing and Evaluating Substance Use Treatment Utilization Estimates from the National Survey on Drug Use and Health and Other Data Sources. In: CBHSQ Data Review. Rockville (MD)2014:1120.

60. Slavova S, Costich JF, Bunn TL, et al. Heroin and fentanyl overdoses in Kentucky: Epidemiology and surveillance. Int J Drug Policy. 2017;46:120-129.

61. Martins SS, Sampson L, Cerda M, Galea S. Worldwide Prevalence and Trends in Unintentional Drug Overdose: A Systematic Review of the Literature. Am J Public Health. 2015;105(11):e29-49.

62. Mars SG, Bourgois P, Karandinos G, Montero F, Ciccarone D. "Every 'never' I ever said came true": transitions from opioid pills to heroin injecting. Int J Drug Policy. 2014;25(2):257-266. 
63. Cerda M, Santaella J, Marshall BD, Kim JH, Martins SS. Nonmedical

Prescription Opioid Use in Childhood and Early Adolescence Predicts Transitions to Heroin Use in Young Adulthood: A National Study. J Pediatr.

2015;167(3):605-612 e601-602.

64. Kennare R, Heard A, Chan A. Substance use during pregnancy: risk factors and obstetric and perinatal outcomes in South Australia. Aust N Z J Obstet Gynaecol. 2005;45(3):220-225.

65. Fischer B, Rehm J, Brissette S, et al. Illicit opioid use in Canada: comparing social, health, and drug use characteristics of untreated users in five cities (OPICAN study). J Urban Health. 2005;82(2):250-266.

66. Mitchell CM, Beals J, Novins DK, Spicer P, American Indian Service Utilization PER, Protective Factors Project T. Drug use among two American Indian populations: prevalence of lifetime use and DSM-IV substance use disorders. Drug Alcohol Depend. 2003;69(1):29-41.

67. Lankenau SE, Teti M, Silva K, Bloom JJ, Harocopos A, Treese M. Initiation into Prescription Opioid Misuse among Young Injection Drug Users. The International journal on drug policy. 2012;23(1):37-44.

68. Edlund MJ, Forman-Hoffman VL, Winder CR, et al. Opioid abuse and depression in adolescents: Results from the National Survey on Drug Use and Health. Drug Alcohol Depend. 2015;152:131-138.

69. Gonek M, Akbarali HI, Henderson G, Dewey WL. Reversal of oxycodone and hydrocodone tolerance by diazepam. Brain Res. 2017;1674:84-90.

70. Guerrieri D, Rapp E, Roman M, Thelander G, Kronstrand R. Acrylfentanyl: Another new psychoactive drug with fatal consequences. Forensic Sci Int. 2017;277:e21-e29.

71. Gunja N. The clinical and forensic toxicology of Z-drugs. J Med Toxicol. 2013;9(2):155-162.

72. Guerrieri D, Rapp E, Roman M, Druid H, Kronstrand R. Postmortem and Toxicological Findings in a Series of Furanylfentanyl-Related Deaths. J Anal Toxicol. 2017;41(3):242-249.

73. Mounteney J, Giraudon I, Denissov G, Griffiths P. Fentanyls: Are we missing the signs? Highly potent and on the rise in Europe. Int J Drug Policy. 2015;26(7):626-631.

74. Algren DA, Monteilh CP, Punja M, et al. Fentanyl-associated fatalities among illicit drug users in Wayne County, Michigan (July 2005-May 2006). J Med Toxicol. 2013;9(1):106-115. 
75. Jalal H, Buchanich JM, Roberts MS, Balmert LC, Zhang K, Burke DS. Changing dynamics of the drug overdose epidemic in the United States from 1979 through 2016. Science. 2018;361(6408).

76. Ciccarone D. Fentanyl in the US heroin supply: A rapidly changing risk environment. Int J Drug Policy. 2017;46:107-111.

77. Seth P, Scholl L, Rudd RA, Bacon S. Overdose Deaths Involving Opioids, Cocaine, and Psychostimulants — United States, 2015-2016. MMWR Morb Mortal Wkly Rep. 2018;67(12):10.

78. Center for Behavioral Health Statistics and Quality. Behavioral health trends in the United States: Results from the 2014 National Survey on Drug Use and Health. 2015.

79. Substance Abuse and Mental Health Services Administration. Key substance use and mental health indicators in the United States: Results from the 2017 National Survey on Drug Use and Health. Rockville, MD: Center for Behavioral Health Statistics and Quality, Substance Abuse and Mental Health Services Administration,;2018.

80. McCabe SE, West BT, Veliz P, McCabe VV, Stoddard SA, Boyd CJ. Trends in Medical and Nonmedical Use of Prescription Opioids Among US Adolescents: 1976-2015. Pediatrics. 2017;139(4).

81. Singhal A, Tien YY, Hsia RY. Racial-Ethnic Disparities in Opioid Prescriptions at Emergency Department Visits for Conditions Commonly Associated with Prescription Drug Abuse. PLoS One. 2016;11(8):e0159224.

82. Dineen KK, DuBois JM. Between a Rock and a Hard Place: Can Physicians Prescribe Opioids to Treat Pain Adequately While Avoiding Legal Sanction. American journal of law \& medicine. 2016;42(1):7-52.

83. Krieger N. Discrimination and health inequities. Int J Health Serv. 2014;44(4):643-710.

84. Netherland J, Hansen H. White opioids: Pharmaceutical race and the war on drugs that wasn't. Biosocieties. 2017;12(2):217-238.

85. Ho JY. The Contribution of Drug Overdose to Educational Gradients in Life Expectancy in the United States, 1992-2011. Demography. 2017;54(3):11751202.

86. Jones CM. The paradox of decreasing nonmedical opioid analgesic use and increasing abuse or dependence - An assessment of demographic and substance use trends, United States, 2003-2014. Addictive Behaviors. 2017;65:229-235. 
87. Han B, Compton WM, Blanco C, Crane E, Lee J, Jones CM. Prescription opioid use, misuse, and use disorders in u.s. adults: 2015 national survey on drug use and health. Annals of Internal Medicine. 2017;167(5):293-301.

88. Han B, Compton WM, Jones CM, Cai R. Nonmedical prescription opioid use and use disorders among adults aged 18 through 64 years in the united states, 20032013. JAMA. 2015;314(14):1468-1478.

89. Ghertner R, Groves L. The Opioid Crisis and Economic Opportunity: Geographic and Economic Trends. In: Department of Health and Human Services, ed. Washington, D.C.2018.

90. Substance Abuse and Mental Health Services Administration. 2016-2017 National Survey on Drug Use and Health National Maps of Prevalence Estimates, by State. 2018.

91. Palamar JJ, Rutherford C, Keyes KM. "Flakka" use among high school seniors in the United States. Drug Alcohol Depend. 2019;196:86-90.

92. Keyes K, Martins S, Hasin D. Past 12-month and lifetime comorbidity and polydrug use of ecstasy users among young adults in the United States: results from the National Epidemiologic Survey on Alcohol and Related Conditions. Drug Alcohol Depend. 2008;97(1-2):139-149.

93. Lankenau SE, Schrager SM, Silva K, et al. Misuse of Prescription and Illicit Drugs Among High-risk Young Adults in Los Angeles and New York. Journal of Public Health Research. 2012a;1(1):22-30.

94. Grigsby TJ, Howard JT. Prescription opioid misuse and comorbid substance use: Past 30-day prevalence, correlates and co-occurring behavioral indicators in the 2016 National Survey on Drug Use and Health. 2019;0(0).

95. Peavy KM, Banta-Green CJ, Kingston S, Hanrahan M, Merrill JO, Coffin PO. "Hooked on" Prescription-Type Opiates Prior to Using Heroin: Results from a Survey of Syringe Exchange Clients. Journal of Psychoactive Drugs. 2012;44(3):259-265.

96. Novak SP, Kral AH. Comparing injection and non-injection routes of administration for heroin, methamphetamine, and cocaine users in the United States. Journal of addictive diseases. 2011;30(3):248-257.

97. Broz D, Zibbell J, Foote C, et al. Multiple injections per injection episode: Highrisk injection practice among people who injected pills during the 2015 HIV outbreak in Indiana. International Journal of Drug Policy. 2018;52:97-101.

98. Zibbell JE, Asher AK, Patel RC, et al. Increases in Acute Hepatitis C Virus Infection Related to a Growing Opioid Epidemic and Associated Injection Drug Use, United States, 2004 to 2014. 2018;108(2):175-181. 
99. Monga N, Rehm J, Fischer B, et al. Using latent class analysis (LCA) to analyze patterns of drug use in a population of illegal opioid users. Drug and Alcohol Dependence. 2007;88(1):1-8.

100. Kuramoto SJ, Bohnert ASB, Latkin CA. Understanding subtypes of inner-city drug users with a latent class approach. Drug and alcohol dependence. 2011;118(2-3):237-243.

101. Zinberg NE. Drug, set, and setting: the basis for controlled intoxicant use. New Haven: Yale University Press; 1984.

102. Hartogsohn I. Constructing drug effects: A history of set and setting. Drug Science, Policy and Law. 2017;3.

103. Reier CE, Nusbaum LM, Anderson RJ. Ethyl alcohol and morphine: a dissociative anesthetic technic. Anesth Analg. 1971;50(6):960-967.

104. Oswald IAN. Drugs and sleep. Pharmacological Reviews. 1968;20(4):273.

105. National Institute on Alcohol Abuse and Alcoholism. Harmful interactions: Mixing alcohol with medicines. In. Bethesda, MD2014.

106. Gudin JA, Mogali S, Jones JD, Comer SD. Risks, management, and monitoring of combination opioid, benzodiazepines, and/or alcohol use. Postgrad Med. 2013;125(4):115-130.

107. Witkiewitz K, Vowles KE. Alcohol and Opioid Use, Co-Use, and Chronic Pain in the Context of the Opioid Epidemic: A Critical Review. Alcohol Clin Exp Res. 2018;42(3):478-488.

108. National Institute on Drug Abuse. Benzodiazepines and Opioids. 2018; https://www.drugabuse.gov/drugs-abuse/opioids/benzodiazepines-opioids\#graph. Accessed July 17, 2018.

109. Institute of Medicine - Division of Health Care Services. An overview of sleep and medication. In: National Academy of Sciences, ed. Report of a study: sleeping pills, insomnia, and medical practice. Washington: National Academy of Sciences; 1979:27-46.

110. Pirnay S, Borron SW, Giudicelli CP, Tourneau J, Baud FJ, Ricordel I. A critical review of the causes of death among post-mortem toxicological investigations: analysis of 34 buprenorphine-associated and 35 methadone-associated deaths. Addiction. 2004;99(8):978-988.

111. Hwang CS, Kang EM, Kornegay CJ, Staffa JA, Jones CM, McAninch JK. Trends in the Concomitant Prescribing of Opioids and Benzodiazepines, 2002-2014. American Journal of Preventive Medicine. 2016;51(2):151-160. 
112. Ditre JW, Brandon TH, Zale EL, Meagher MM. Pain, nicotine, and smoking: research findings and mechanistic considerations. Psychological bulletin. 2011;137(6):1065-1093.

113. Chapman SLC, Wu L-T. Associations between cigarette smoking and pain among veterans. Epidemiologic reviews. 2015;37(1):86-102.

114. Shi Y, Weingarten TN, Mantilla CB, Hooten WM, Warner DO. Smoking and pain: pathophysiology and clinical implications. Anesthesiology. 2010;113(4):977-992.

115. Zvolensky MJ, McMillan K, Gonzalez A, Asmundson GJG. Chronic pain and cigarette smoking and nicotine dependence among a representative sample of adults. Nicotine \& tobacco research : official journal of the Society for Research on Nicotine and Tobacco. 2009;11(12):1407-1414.

116. Mackey I, Sharifzadeh Y, Sturgeon J, Mackey S. Cigarette smoking is a predictor of opioid use in a tertiary care pain clinic sample: a Collaborative Health Outcomes Information Registry (CHOIR) study. The Journal of Pain. 2016;17(4):S15.

117. Rogal SS, Winger D, Bielefeldt K, Szigethy E. Pain and opioid use in chronic liver disease. Digestive diseases and sciences. 2013;58(10):2976-2985.

118. Dobscha SK, Morasco BJ, Duckart JP, Macey T, Deyo RA. Correlates of prescription opioid initiation and long-term opioid use in veterans with persistent pain. The Clinical journal of pain. 2013;29(2):102-108.

119. Vihavainen T, Piltonen M, Tuominen RK, Korpi ER, Ahtee L. Morphine-nicotine interaction in conditioned place preference in mice after chronic nicotine exposure. European Journal of Pharmacology. 2008;587(1):169-174.

120. Hooten WM, Shi Y, Gazelka HM, Warner DO. The effects of depression and smoking on pain severity and opioid use in patients with chronic pain. Pain. 2011;152(1):223-229.

121. John WS, Zhu H, Mannelli P, et al. Prevalence and patterns of opioid misuse and opioid use disorder among primary care patients who use tobacco. Drug Alcohol Depend. 2019;194:468-475.

122. Fu Q, Vaughn MG, Wu L-T, Heath AC. Psychiatric correlates of snuff and chewing tobacco use. PloS one. 2014;9(12):e113196-e113196.

123. National Institute on Drug Abuse. Cocaine. 2018; https://www.drugabuse.gov/publications/drugfacts/cocaine, 2019.

124. Leri F, Bruneau J, Stewart J. Understanding polydrug use: review of heroin and cocaine co-use. Addiction. 2003;98(1):7-22. 
125. Henningfield JE, Luckas SE, Bigelow GE. Human studies of drug as reinforcers. In: Goldberg SR, Stolerman IP, eds. Behavioral Analysis of Drug Dependence. Orlando: Academic Press; 1986.

126. The National Institute on Drug Abuse Blog Team. Real Teens Ask About Speedballs. 2013; https://teens.drugabuse.gov/blog/post/real-teens-ask-aboutspeedballs, 2019.

127. Walsh SL, Sullivan JT, Preston KL, Garner JE, Bigelow GE. Effects of naltrexone on response to intravenous cocaine, hydromorphone and their combination in humans. The Journal of pharmacology and experimental therapeutics. 1996;279(2):524-538.

128. Foltin RW, Fischman MW. Effects of methadone or buprenorphine maintenance on the subjective and reinforcing effects of intravenous cocaine in humans. The Journal of pharmacology and experimental therapeutics. 1996;278(3):1153-1164.

129. Stine SM, Satel S, Kosten T. Cocaine Precipitation of Patient-Identified Opiate Withdrawal. 1993;2(3):255-258.

130. Malow RM, West JA, Corrigan SA, Pena JM, Criss Lott WVAMCNOLUSA. Cocaine and speedball users: Differences in psychopathology. Journal of Substance Abuse Treatment. 1992;9(4):287-291.

131. Hunt DE, Lipton DS, Goldsmith D, Strug D. Street pharmacology: Uses of cocaine and heroin in the treatment of addiction. Drug and Alcohol Dependence. 1984;13(4):375-387.

132. Bronshtein T. Interactive: Explore how illegal drugs have become cheaper and more potent over time. StatNews.com 2016.

133. National Institute on Drug Abuse. Hallucinogens. 2016; https://www.drugabuse.gov/publications/drugfacts/hallucinogens, 2019.

134. National Institute on Drug Abuse. Inhalants. 2012; https://www.drugabuse.gov/publications/research-reports/inhalants/what-areinhalants, 2019.

135. Wu L-T, Woody GE, Yang C, Blazer DG. How do prescription opioid users differ from users of heroin or other drugs in psychopathology: results from the National Epidemiologic Survey on Alcohol and Related Conditions. Journal of addiction medicine. 2011;5(1):28-35.

136. Pisano VD, Putnam NP, Kramer HM, Franciotti KJ, Halpern JH, Holden SC. The association of psychedelic use and opioid use disorders among illicit users in the United States. Journal of Psychopharmacology. 2017;31(5):606-613. 
137. Zhang S. Patients Are Ditching Opioid Pills for Weed: Can marijuana help solve the opioid epidemic? The Atlantic 2017.

138. Miller G. Could pot help solve the U.S. opioid epidemic? Science. 2016.

139. Hayes MJ, Brown MS. Legalization of medical marijuana and incidence of opioid mortality. JAMA Internal Medicine. 2014;174(10):1673-1674.

140. Bachhuber MA, Saloner B, Cunningham CO, Barry CL. Medical cannabis laws and opioid analgesic overdose mortality in the united states, 1999-2010. JAMA Internal Medicine. 2014;174(10):1668-1673.

141. Powell D, Pacula RL, Jacobson M. Do Medical Marijuana Laws Reduce Addictions and Deaths Related to Pain Killers? In. Washington, D.C.: The National Bureau of Economic Research; 2015.

142. Vigil JM, Stith SS, Adams IM, Reeve AP. Associations between medical cannabis and prescription opioid use in chronic pain patients: A preliminary cohort study. PloS one. 2017;12(11):e0187795-e0187795.

143. Olfson M, Wall MM, Liu S-M, Blanco C. Cannabis Use and Risk of Prescription Opioid Use Disorder in the United States. The American journal of psychiatry. 2018;175(1):47-53.

144. Lopez-Quintero C, Perez de los Cobos J, Hasin DS, et al. Probability and predictors of transition from first use to dependence on nicotine, alcohol, cannabis, and cocaine: results of the National Epidemiologic Survey on Alcohol and Related Conditions (NESARC). Drug Alcohol Depend. 2011;115(1-2):120130 .

145. National Institute on Drug Abuse. Prescription stimulants. 2018; https://www.drugabuse.gov/publications/drugfacts/prescription-stimulants, 2019.

146. Wei Y-JJ, Zhu Y, Liu W, Bussing R, Winterstein AG. Prevalence of and Factors Associated With Long-term Concurrent Use of Stimulants and Opioids Among Adults With Attention-Deficit/Hyperactivity DisorderLong-term Concurrent Use of Stimulants and Opioids Among Adults With ADHDLong-term Concurrent Use of Stimulants and Opioids Among Adults With ADHD. JAMA Network Open. 2018;1(4):e181152-e181152.

147. Lord S, Downs G, Furtaw P, et al. Nonmedical use of prescription opioids and stimulants among student pharmacists. Journal of the American Pharmacists Association. 2009;49(4):519-528.

148. Perlmutter AS, Conner SC, Savone M, Kim JH, Segura LE, Martins SS. Is employment status in adults over 25 years old associated with nonmedical prescription opioid and stimulant use? Social psychiatry and psychiatric epidemiology. 2017;52(3):291-298. 
149. McCabe SE, Veliz PT, Boyd CJ, Schepis TS, McCabe VV, Schulenberg JE. A prospective study of nonmedical use of prescription opioids during adolescence and subsequent substance use disorder symptoms in early midlife. Drug Alcohol Depend. 2018;194:377-385.

150. Centers for Disease Control and Prevention. Module 5: Assessing and Addressing Opioid Use Disorder (OUD). 2018; https://www.cdc.gov/drugoverdose/training/oud/accessible/index.html.

151. Substance Abuse and Mental Health Services Administration. Managing Chronic Pain in Adults With or in Recovery From Substance Use Disorders. In. Rockville, MD: Substance Abuse and Mental Health Services Administration,; 2011.

152. Hasin DS, O'Brien CP, Auriacombe M, et al. DSM-5 criteria for substance use disorders: recommendations and rationale. The American journal of psychiatry. 2013;170(8):834-851.

153. Center for Behavioral Health Statistics and Quality. 2015 National Survey on Drug Use and Health Public Use File Codebook. In. Rockville, MD: Substance Abuse and Mental Health Services Administration,; 2016.

154. Donroe JH, Tetrault JM. Substance Use, Intoxication, and Withdrawal in the Critical Care Setting. Crit Care Clin. 2017;33(3):543-558.

155. Degenhardt L, Bucello C, Mathers B, et al. Mortality among regular or dependent users of heroin and other opioids: a systematic review and meta-analysis of cohort studies. Addiction. 2011;106(1):32-51.

156. Wu L-T, Zhu H, Swartz MS. Treatment utilization among persons with opioid use disorder in the United States. Drug and alcohol dependence. 2016;169:117-127.

157. Schepis TS, Hakes JK. Non-medical prescription use increases the risk for the onset and recurrence of psychopathology: results from the National Epidemiological Survey on Alcohol and Related Conditions. Addiction. 2011;106(12):2146-2155.

158. Olfson M, Wall M, Wang S, Crystal S, Blanco C. Service Use Preceding OpioidRelated Fatality. 2018;175(6):538-544.

159. Miller T, Novak SP, Galvin DM, Spicer RS, Cluff L, Kasat S. School and work status, drug-free workplace protections, and prescription drug misuse among Americans ages 15-25. J Stud Alcohol Drugs. 2015;76(2):195-203.

160. Kidorf M, Hollander JR, King VL, Brooner RK. Increasing employment of opioid dependent outpatients: an intensive behavioral intervention. Drug and Alcohol Dependence. 1998;50(1):73-80. 
161. Rigg KK, Monnat SM. Urban vs. rural differences in prescription opioid misuse among adults in the United States: informing region specific drug policies and interventions. Int J Drug Policy. 2015;26(5):484-491.

162. Hegmann KT, Weiss MS, Bowden K, et al. ACOEM Practice Guidelines: Opioids and Safety-Sensitive Work. 2014;56(7):e46-e53.

163. Glied S, Kronick R. The Value of Health Insurance: Few of the Uninsured Have Adequate Resources to Pay Potential Hospital Bills. 2011; https://aspe.hhs.gov/pdf-report/value-health-insurance-few-uninsured-haveadequate-resources-pay-potential-hospital-bills.

164. Christopher AS, McCormick D, Woolhandler S, Himmelstein DU, Bor DH, Wilper AP. Access to Care and Chronic Disease Outcomes Among MedicaidInsured Persons Versus the Uninsured. American journal of public health. 2016;106(1):63-69.

165. Woolhandler S, Himmelstein DU. The Relationship of Health Insurance and Mortality: Is Lack of Insurance Deadly?Relationship of Health Insurance and Mortality. Annals of Internal Medicine. 2017;167(6):424-431.

166. Hasegawa K, Brown DF, Tsugawa Y, Camargo CA, Jr. Epidemiology of emergency department visits for opioid overdose: a population-based study. Mayo Clin Proc. 2014;89(4):462-471.

167. Paulozzi LJ, Xi Y. Recent changes in drug poisoning mortality in the United States by urban-rural status and by drug type. Pharmacoepidemiol Drug Saf. 2008;17(10):997-1005.

168. Young A, Havens J, Leukefeld C. A Comparison of Rural and Urban Nonmedical Prescription Opioid Users' Lifetime and Recent Drug Use. The American Journal of Drug and Alcohol Abuse. 2012;38(3):220-227.

169. Glasheen C, Forman-Hoffman VL, Williams J. Residential Mobility, Transience, Depression, and Marijuana Use Initiation Among Adolescents and Young Adults. Subst Abuse. 2017;11:1178221817711415.

170. Spinner GF, Leaf PJ. Homelessness and drug abuse in New Haven. Hospital \& community psychiatry. 1992;43(2):166-168.

171. Riley ED, Shumway M, Knight KR, Guzman D, Cohen J, Weiser SD. Risk factors for stimulant use among homeless and unstably housed adult women. Drug and alcohol dependence. 2015;153:173-179.

172. Palepu A, Marshall BDL, Lai C, Wood E, Kerr T. Addiction treatment and stable housing among a cohort of injection drug users. PloS one. 2010;5(7):e11697e11697. 
173. Baggett TP, Hwang SW, O'Connell JJ, et al. Mortality among homeless adults in Boston: shifts in causes of death over a 15-year period. JAMA internal medicine. 2013;173(3):189-195.

174. Katherine H. Shelton, Pamela J. Taylor, Adrian Bonner, Marianne van den Bree Risk Factors for Homelessness: Evidence From a Population-Based Study. Psychiatric Services. 2009;60(4):465-472.

175. Forman-Hoffman VL, Glasheen C, Ridenour TA. Residential Transience and Substance Use Disorder Are Independently Associated with Suicidal Thoughts, Plans, and Attempts in a Nationally Representative Sample of U.S. Adults. Suicide Life Threat Behav. 2018;48(4):401-412.

176. Wang L, Min JE, Krebs E, et al. Polydrug use and its association with drug treatment outcomes among primary heroin, methamphetamine, and cocaine users. The International journal on drug policy. 2017;49:32-40.

177. Center for Behavioral Health Statistics and Quality. 2016 National Survey on Drug Use and Health (NSDUH): Screener Specifications for Programming (English Version). Rockville, MD2015. HHS Publication No. SMA 16-4984, NSDUH Series H-51.

178. Center for Behavioral Health Statistics and Quality. National Survey on Drug Use and Health Final Analytic File Codebook. In. Rockville, MD: Substance Abuse and Mental Health Services Administration,; 2002-2017.

179. Center for Behavioral Health Statistics and Quality. National Survey on Drug Use and Health: 2014 and 2015 Redesign Changes. In. Rockville, MD: Substance Abuse and Mental Health Services Administration; 2015.

180. Manchikanti L, Singh A. Therapeutic opioids: a ten-year perspective on the complexities and complications of the escalating use, abuse, and nonmedical use of opioids. Pain Physician. 2008;11(2 Suppl):S63-88.

181. Hedden S, Gfroerer J, Barker P, et al. Comparison of NSDUH Mental Health Data and Methods with Other Data Sources. In: CBHSQ Data Review. Rockville (MD)2012:1-19.

182. Kerridge BT, Saha TD, Smith S, et al. Dimensionality of hallucinogen and inhalant/solvent abuse and dependence criteria: implications for the Diagnostic and Statistical Manual of Mental Disorders-Fifth Edition. Addict Behav. 2011;36(9):912-918.

183. Kuehn BM. Alarming nonfatal overdose rates found for opioids, sedatives, and tranquilizers. JAMA. 2010;303(20):2020-2021. 
184. Wu LT, Parrott AC, Ringwalt CL, Yang C, Blazer DG. The variety of ecstasy/MDMA users: results from the National Epidemiologic Survey on alcohol and related conditions. Am J Addict. 2009;18(6):452-461.

185. Heinz AJ, Wu J, Witkiewitz K, Epstein DH, Preston KL. Marriage and relationship closeness as predictors of cocaine and heroin use. Addictive behaviors. 2009;34(3):258-263.

186. Lex BW. Alcohol and other drug abuse among women. Alcohol Health \& Research World. 1994;18(3):212.

187. Kaestner R. The Effects of Cocaine and Marijuana Use on Marriage and Marital Stability. In. Cambridge: National Bureau of Economic Research, Inc.; 1995:5038.

188. Population Reference Bureau. U.S. Counties by Rural-Urban Continuum Codes in 2003. 2004;

https://www.prb.org/uscountiesbyruralurbancontinuumcodesin2003pdf10mb/, 2018.

189. University of California LA, Institute for Digital Research and Education. Applied Survey Data Analysis using SAS 9.4. 2018; https://stats.idre.ucla.edu/sas/seminars/sas-survey/.

190. Akinyede O, Soyemi K. Joinpoint regression analysis of pertussis crude incidence rates, Illinois, 1990-2014. Am J Infect Control. 2016;44(12):1732-1733.

191. National Cancer Institute. Joinpoint Trend Analysis Software. 2019; https://surveillance.cancer.gov/joinpoint/, 2019.

192. National Cancer Institute. How Joinpoint Selects the Final Model. 2019; https://surveillance.cancer.gov/help/joinpoint/setting-parameters/method-andparameters-tab/model-selection-method, 2019.

193. McCutcheon AL. Latent class analysis. Newbury Park: Sage Publications; 1987.

194. Collins LM, Lanza ST. Latent class and latent transition analysis : with applications in the social behavioral, and health sciences. Hoboken, N.J.: Wiley; 2010.

195. Muthén B, Muthén LK. Integrating person-centered and variable-centered analyses: growth mixture modeling with latent trajectory classes. Alcohol Clin Exp Res. 2000;24(6):882-891.

196. Rao JNK, Scott AJ. On Chi-Squared Tests for Multiway Contingency Tables with Cell Proportions Estimated from Survey Data. The Annals of Statistics. 1984;12(1):46-60. 
197. Celeux G, Soromenho G. An entropy criterion for assessing the number of clusters in a mixture model. Journal of Classification. 1996;13(2):195-212.

198. Tein J-Y, Coxe S, Cham H. Statistical Power to Detect the Correct Number of Classes in Latent Profile Analysis. Structural equation modeling : a multidisciplinary journal. 2013;20(4):640-657.

199. Tavitian-Exley I, Boily M-C, Heimer R, Uusküla A, Levina O, Maheu-Giroux M. Polydrug Use and Heterogeneity in HIV Risk Among People Who Inject Drugs in Estonia and Russia: A Latent Class Analysis. AIDS and behavior. 2018;22(4):1329-1340.

200. Roy E, Richer I, Arruda N, Vandermeerschen J, Bruneau J. Patterns of cocaine and opioid co-use and polyroutes of administration among street-based cocaine users in Montreal, Canada. Int J Drug Policy. 2013;24(2):142-149.

201. Dasgupta N, Beletsky L, Ciccarone D. Opioid Crisis: No Easy Fix to Its Social and Economic Determinants. American journal of public health. 2018;108(2):182-186.

202. Nylund KL, Asparouhov T, Muthén BO. Deciding on the Number of Classes in Latent Class Analysis and Growth Mixture Modeling: A Monte Carlo Simulation Study. Structural Equation Modeling: A Multidisciplinary Journal. 2007;14(4):535-569.

203. Muthén BO, Muthén LK, Asparouhov T. Regression and Mediation Analysis Using Mplus. Muthén \& Muthén; 2016.

204. Kelly PJ, Robinson LD, Baker AL, et al. Polysubstance use in treatment seekers who inject amphetamine: Drug use profiles, injecting practices and quality of life. Addictive Behaviors. 2017;71:25-30.

205. Zoorob M. Polydrug epidemiology: Benzodiazepine prescribing and the drug overdose epidemic in the United States. Pharmacoepidemiology and Drug Safety. 2018;0(0).

206. Winstanley EL, Clark A, Feinberg J, Wilder CM. Barriers to implementation of opioid overdose prevention programs in Ohio. Substance abuse. 2016;37(1):4246.

207. National Institute on Drug Abuse. Cascade of Care model recommended for opioid crisis. 2019; https://www.drugabuse.gov/news-events/newsreleases/2019/01/cascade-care-model-recommended-opioid-crisis, 2019.

208. The Epidemic of Prescription Drug and Heroin Abuse in the United States [press release]. Washington, D.C.: Executive Office of the President: Office of National Drug Control Policy2016. 
209. Elder JW, DePalma G, Pines JM. Optimal Implementation of Prescription Drug Monitoring Programs in the Emergency Department. The western journal of emergency medicine. 2018;19(2):387-391.

210. Evans WN, Lieber E, Power P. How the Reformulation of OxyContin Ignited the Heroin Epidemic. In. Notre Dame, IN2018.

211. Kozlowski LT. Origins in the USA in the 1980s of the warning that smokeless tobacco is not a safe alternative to cigarettes: a historical, documents-based assessment with implications for comparative warnings on less harmful tobacco/nicotine products. Harm Reduction Journal. 2018;15(1):21.

212. IARC Working Group on the Evaluation of Carcinogenic Risk to Humans. Smokeless Tobacco and Some Tobacco-specific N-Nitrosamines. In. Lyon, France: International Agency for Research on Cancer; 2007.

213. Charles A S. Developing universal electronic medical records. Gastroenterology \& hepatology. 2008;4(3):193-195.

214. Harpaz J. Electronic Health Records: A Promising New Way To Fight the Opioid Epidemic. 2019; https://www.forbes.com/sites/joeharpaz/2019/03/15/electronichealth-records-a-promising-new-way-to-fight-the-opioidepidemic/\#14a0075d3c8c, 2019.

215. Stucke RS, Kelly JL, Mathis KA, Hill MV, Barth RJ, Jr. Association of the Use of a Mandatory Prescription Drug Monitoring Program With Prescribing Practices for Patients Undergoing Elective SurgeryPrescription Drug Monitoring Program and Prescribing Practices for Patients Undergoing Elective SurgeryPrescription Drug Monitoring Program and Prescribing Practices for Patients Undergoing Elective Surgery. JAMA Surgery. 2018;153(12):1105-1110.

216. Mannelli P, Wu LT, Peindl KS, Gorelick DA. Smoking and opioid detoxification: behavioral changes and response to treatment. Nicotine Tob Res. 2013;15(10):1705-1713.

217. Kampman KM. New medications for the treatment of cocaine dependence. Psychiatry (Edgmont (Pa : Township)). 2005;2(12):44-48.

218. Throckmorton DC, Gottlieb S, Woodcock J. The FDA and the Next Wave of Drug Abuse - Proactive Pharmacovigilance. New England Journal of Medicine. 2018;379(3):205-207.

219. Moore BA, Fiellin DA, Cutter CJ, et al. Cognitive Behavioral Therapy Improves Treatment Outcomes for Prescription Opioid Users in Primary Care Buprenorphine Treatment. Journal of substance abuse treatment. 2016;71:54-57.

220. Cox J, Zgierska AE, Burzinski CA, et al. Mindfulness Meditation and Cognitive Behavioral Therapy Intervention Reduces Pain Severity and Sensitivity in Opioid- 
Treated Chronic Low Back Pain: Pilot Findings from a Randomized Controlled Trial. Pain Medicine. 2016;17(10):1865-1881.

221. Pemovska T, Bigenzahn JW, Superti-Furga G. Recent advances in combinatorial drug screening and synergy scoring. Current Opinion in Pharmacology. 2018;42:102-110.

222. McAlister S. 'The Living Room' welcoming people in crisis, changing lives. 2019; https://www.whas11.com/article/news/the-living-room-welcoming-people-incrisis-changing-lives/417-c68d3dbe-f7f9-4d17-85b0-ed9a694c6dd0, 2019.

223. Center for Behavioral Health Statistics and Quality. 2014 National Survey on Drug Use and Health: Methodological summary and definitions. In. Rockville, MD: Substance Abuse and Mental Health Services Administration; 2015.

224. Humphreys K. The federal government is systematically undercounting heroin users. The Washington Post 2017.

225. Prendergast ML. Interventions to promote successful re-entry among drugabusing parolees. Addiction science \& clinical practice. 2009;5(1):4-13.

226. Teeters JB, Lancaster CL, Brown DG, Back SE. Substance use disorders in military veterans: prevalence and treatment challenges. Substance abuse and rehabilitation. 2017;8:69-77.

227. Moos RH, Moos BS, Andrassy JM. Outcomes of four treatment approaches in community residential programs for patients with substance use disorders. Psychiatr Serv. 1999;50(12):1577-1583.

228. Tein JY, Coxe S, Cham H. Statistical Power to Detect the Correct Number of Classes in Latent Profile Analysis. Struct Equ Modeling. 2013;20(4):640-657.

229. Dziak JJ, Lanza ST, Tan X. Effect Size, Statistical Power and Sample Size Requirements for the Bootstrap Likelihood Ratio Test in Latent Class Analysis. Struct Equ Modeling. 2014;21(4):534-552.

230. Goldman JE, Waye KM, Periera KA, Krieger MS, Yedinak JL, Marshall BDL. Perspectives on rapid fentanyl test strips as a harm reduction practice among young adults who use drugs: a qualitative study. Harm reduction journal. 2019;16(1):3-3.

231. Williams AR, Nunes EV, Bisaga A, Levin FR, Olfson M. Development of a Cascade of Care for responding to the opioid epidemic. The American Journal of Drug and Alcohol Abuse. 2019;45(1):1-10. 
APPENDIX A: Supplemental Tables (ST) 
ST 4.1 Joinpoint regression results of longitudinal changes in prevalence of PWUO, NSDUH 2002 to $2017^{\mathrm{a}}$

\begin{tabular}{|c|c|c|c|c|c|c|}
\hline Segment & $\begin{array}{c}\text { Lower } \\
\text { endpoint }\end{array}$ & $\begin{array}{c}\text { Upper } \\
\text { endpoint }\end{array}$ & Slope & SE & $\begin{array}{c}\text { Test } \\
\text { statistic } \\
(\mathbf{t})\end{array}$ & p-value \\
\hline $\mathbf{1}$ & 2002 & 2010 & $0.028^{\wedge}$ & 0.01023 & 2.72 & 0.020 \\
\hline $\mathbf{2}$ & 2010 & 2017 & $-0.117^{\wedge}$ & 0.01019 & -11.51 & $<0.001$ \\
\hline
\end{tabular}

Abbreviations: $\mathrm{SE}=$ standard error

${ }^{a}$ Two continuous variables were used to perform the joinpoint regression: the weighted prevalence of past month PWUO and survey year.

${ }^{\wedge}$ Slope is significantly different from zero at alpha $=0.05$.

ST 4.2 | Joinpoint regression results of longitudinal changes in prevalence of past month PPR-only use group, NSDUH 2002 to $2017^{\mathrm{a}}$

\begin{tabular}{|c|c|c|c|c|c|c|}
\hline Segment & $\begin{array}{c}\text { Lower } \\
\text { endpoint }\end{array}$ & $\begin{array}{c}\text { Upper } \\
\text { endpoint }\end{array}$ & Slope & SE & $\begin{array}{c}\text { Test } \\
\text { statistic } \\
(\mathbf{t})\end{array}$ & p-value \\
\hline $\mathbf{1}$ & 2002 & 2017 & $-0.455^{\wedge}$ & 0.099 & -4.59 & 0.0004 \\
\hline
\end{tabular}

Abbreviations: $\mathrm{SE}=$ standard error; $\mathrm{CI}=$ confidence interval

${ }^{a}$ Two continuous variables were used to perform the joinpoint regression: the weighted prevalence of past month PPR-only use group and survey year.

${ }^{\wedge}$ Slope is significantly different from zero at alpha $=0.05$.

ST 4.3 Joinpoint regression results of longitudinal changes in prevalence of past month heroin-only use group, NSDUH 2002 to $2017^{\mathrm{a}}$

\begin{tabular}{|c|c|c|c|c|c|c|}
\hline Segment & $\begin{array}{c}\text { Lower } \\
\text { endpoint }\end{array}$ & $\begin{array}{c}\text { Upper } \\
\text { endpoint }\end{array}$ & Slope & SE & $\begin{array}{c}\text { Test } \\
\text { statistic } \\
(\mathbf{t})\end{array}$ & p-value \\
\hline $\mathbf{1}$ & 2002 & 2010 & 0.051 & 0.245 & 0.21 & 0.84 \\
\hline $\mathbf{2}$ & 2010 & 2017 & $1.062^{\wedge}$ & 0.218 & 4.87 & $<0.001$ \\
\hline
\end{tabular}

Abbreviations: $\mathrm{SE}=$ standard error; $\mathrm{CI}=$ confidence interval

${ }^{a}$ Two continuous variables were used to perform the joinpoint regression: the weighted prevalence of past month heroin-only use group and survey year.

${ }^{\wedge}$ Slope is significantly different from zero at alpha $=0.05$.

ST 4.4 Joinpoint regression results of longitudinal changes in prevalence of past month combination opioid use group, NSDUH 2002 to $2017^{\mathrm{a}}$

\begin{tabular}{|c|c|c|c|c|c|c|}
\hline Segment & $\begin{array}{c}\text { Lower } \\
\text { endpoint }\end{array}$ & $\begin{array}{c}\text { Upper } \\
\text { endpoint }\end{array}$ & Slope & SE & $\begin{array}{c}\text { Test } \\
\text { statistic } \\
(\mathbf{t})\end{array}$ & p-value \\
\hline $\mathbf{1}$ & 2002 & 2017 & $0.35^{\wedge}$ & 0.013 & 27.83 & $<0.001$ \\
\hline
\end{tabular}

Abbreviations: $\mathrm{SE}=$ standard error; $\mathrm{CI}=$ confidence interval

${ }^{a}$ Two continuous variables were used to perform the joinpoint regression: the weighted prevalence of past month PPR-only use group and survey year.

${ }^{\wedge}$ Slope is significantly different from zero at alpha $=0.05$. 
ST 4.5 | Overall weighted prevalence of reported past month core substances use among PWUO, NSDUH 2002 to $2017(\mathrm{n}=16,985)$

\begin{tabular}{|c|c|c|c|c|c|c|}
\hline \multirow{2}{*}{\multicolumn{2}{|c|}{ Core Substance }} & \multirow{2}{*}{$\mathbf{n}$} & \multirow{2}{*}{$\%$} & \multirow{2}{*}{ SE } & \multicolumn{2}{|c|}{$95 \% \mathrm{CI}$} \\
\hline & & & & & $\mathbf{L L}$ & UL \\
\hline \multirow{2}{*}{ Binge alcohol } & $\begin{array}{c}\text { Binge use in the past } \\
\text { month }\end{array}$ & 5523 & 28.80 & 0.62 & 26.82 & 30.78 \\
\hline & $\begin{array}{l}\text { Never or 'no' binge } \\
\text { use in past month }\end{array}$ & 11462 & 71.20 & 0.62 & 69.22 & 73.18 \\
\hline \multirow{2}{*}{ Heavy alcohol } & $\begin{array}{l}\text { Heavy use in the } \\
\text { past month }\end{array}$ & 5211 & 25.32 & 0.60 & 23.41 & 27.23 \\
\hline & $\begin{array}{l}\text { Never or 'no' heavy } \\
\text { use in past month }\end{array}$ & 11774 & 74.68 & 0.60 & 72.77 & 76.59 \\
\hline \multirow{2}{*}{$\begin{array}{l}\text { Tranquilizers/ } \\
\text { sedatives }\end{array}$} & $\begin{array}{l}\text { Used in the past } \\
\text { month }\end{array}$ & 3860 & 20.91 & 0.40 & 19.64 & 22.17 \\
\hline & $\begin{array}{l}\text { Not used in the past } \\
\text { month }\end{array}$ & 13125 & 79.09 & 0.40 & 77.83 & 80.36 \\
\hline \multirow{2}{*}{ Cigarettes } & $\begin{array}{l}\text { Used in the past } \\
\text { month }\end{array}$ & 11346 & 58.74 & 0.37 & 57.57 & 59.92 \\
\hline & $\begin{array}{l}\text { Not used in the past } \\
\text { month }\end{array}$ & 5639 & 41.26 & 0.37 & 40.08 & 42.43 \\
\hline \multirow{2}{*}{$\begin{array}{l}\text { Other tobacco } \\
\text { products }\end{array}$} & $\begin{array}{l}\text { Used in the past } \\
\text { month }\end{array}$ & 4971 & 23.56 & 0.34 & 22.47 & 24.65 \\
\hline & $\begin{array}{l}\text { Not used in the past } \\
\text { month }\end{array}$ & 12014 & 76.44 & 0.34 & 75.35 & 77.53 \\
\hline \multirow{2}{*}{ Marijuana } & $\begin{array}{l}\text { Used in the past } \\
\text { month }\end{array}$ & 8672 & 40.89 & 0.58 & 39.03 & 42.75 \\
\hline & $\begin{array}{l}\text { Not used in the past } \\
\text { month }\end{array}$ & 8313 & 59.11 & 0.58 & 57.25 & 60.97 \\
\hline \multirow{2}{*}{ Cocaine } & $\begin{array}{l}\text { Used in the past } \\
\text { month }\end{array}$ & 2122 & 11.21 & 0.41 & 9.90 & 12.52 \\
\hline & $\begin{array}{l}\text { Not used in the past } \\
\text { month }\end{array}$ & 14863 & 88.79 & 0.41 & 87.48 & 90.10 \\
\hline \multirow{2}{*}{ Crack } & $\begin{array}{l}\text { Used in the past } \\
\text { month }\end{array}$ & 527 & 3.66 & 0.44 & 2.2588 & 5.06 \\
\hline & $\begin{array}{l}\text { Not used in the past } \\
\text { month }\end{array}$ & 16458 & 96.34 & 0.44 & 94.94 & 97.74 \\
\hline \multirow{2}{*}{$\begin{array}{l}\text { Hallucinogens/ } \\
\text { inhalants }\end{array}$} & $\begin{array}{l}\text { Used in the past } \\
\text { month }\end{array}$ & 1602 & 6.76 & 0.26 & 5.91 & 7.60 \\
\hline & $\begin{array}{l}\text { Not used in the past } \\
\text { month }\end{array}$ & 15383 & 93.24 & 0.26 & 92.40 & 94.09 \\
\hline \multirow{2}{*}{ Stimulants } & $\begin{array}{l}\text { Used in the past } \\
\text { month }\end{array}$ & 1609 & 7.78 & 0.20 & 7.14 & 8.41 \\
\hline & $\begin{array}{l}\text { Not used in the past } \\
\text { month }\end{array}$ & 15376 & 92.23 & 0.20 & 91.59 & 92.86 \\
\hline
\end{tabular}

Abbreviations: $\mathrm{n}=$ number of respondents, $\%=$ weighted prevalence; $\mathrm{SE}=$ standard error; $95 \%$ $\mathrm{CI}=95 \%$ confidence interval; $\mathrm{LL}=$ lower limit; $\mathrm{UL}=$ upper limit 
ST 4.6 Weighted prevalence of reported substance use for all PWUO by period ( $\mathrm{n}=16,985)$, NSDUH 2002 to 2017

\begin{tabular}{|c|c|c|c|c|c|c|c|c|c|c|c|}
\hline \multirow{4}{*}{\multicolumn{2}{|c|}{ Core substances }} & \multicolumn{10}{|c|}{ Period } \\
\hline & & \multicolumn{5}{|c|}{ (1) 2002 to 2005} & \multicolumn{5}{|c|}{ (2) 2006 to 2009} \\
\hline & & \multirow{2}{*}{$\mathbf{n}$} & \multirow{2}{*}{$\%^{a}$} & \multirow{2}{*}{ SE } & \multicolumn{2}{|c|}{$95 \% \mathrm{CI}$} & \multirow{2}{*}{$\mathbf{n}$} & \multirow{2}{*}{$\%^{a}$} & \multirow{2}{*}{ SE } & \multicolumn{2}{|c|}{$95 \% \mathrm{CI}$} \\
\hline & & & & & $\mathbf{L L}$ & $\mathbf{U L}$ & & & & $\mathbf{L} \mathbf{L}$ & $\mathbf{U L}$ \\
\hline \multirow[b]{2}{*}{ Binge alcohol } & Binge use in the past month & 1423 & 28.13 & 1.66 & 22.84 & 33.42 & 1565 & 30.04 & 0.46 & 28.57 & 31.51 \\
\hline & $\begin{array}{l}\text { Never or 'no' binge use in past } \\
\text { month }\end{array}$ & 3034 & 71.87 & 1.66 & 66.58 & 77.16 & 3331 & 69.96 & 0.46 & 68.49 & 71.43 \\
\hline \multirow[b]{2}{*}{ Heavy alcohol } & Heavy use in the past month & 1571 & 28.06 & 1.72 & 22.60 & 33.53 & 1687 & 27.96 & 0.54 & 26.25 & 29.66 \\
\hline & $\begin{array}{l}\text { Never or 'no' heavy use in past } \\
\text { month }\end{array}$ & 2886 & 71.94 & 1.72 & 66.47 & 77.40 & 3300 & 72.04 & 0.54 & 70.34 & 73.75 \\
\hline \multirow{2}{*}{$\begin{array}{l}\text { Tranquilizers/ } \\
\text { sedatives }\end{array}$} & Used in the past month & 1019 & 21.61 & 0.44 & 20.22 & 23.00 & 1166 & 20.50 & 0.30 & 19.53 & 21.46 \\
\hline & Not used in the past month & 3438 & 78.39 & 0.44 & 77.00 & 79.78 & 3821 & 79.50 & 0.30 & 78.54 & 80.47 \\
\hline \multirow{2}{*}{ Cigarettes } & Used in the past month & 3039 & 59.95 & 1.04 & 56.63 & 63.27 & 3439 & 60.57 & 0.61 & 58.62 & 62.52 \\
\hline & Not used in the past month & 1418 & 40.05 & 1.04 & 36.73 & 43.37 & 1548 & 39.43 & 0.61 & 37.48 & 41.38 \\
\hline \multirow{2}{*}{$\begin{array}{c}\text { Other tobacco } \\
\text { products }\end{array}$} & Used in the past month & 1314 & 23.89 & 1.07 & 20.48 & 27.31 & 1523 & 24.74 & 0.33 & 23.68 & 25.80 \\
\hline & Not used in the past month & 3143 & 76.11 & 1.07 & 72.69 & 79.52 & 3464 & 75.26 & 0.33 & 74.20 & 76.32 \\
\hline \multirow{2}{*}{ Marijuana } & Used in the past month & 2287 & 40.54 & 2.33 & 33.14 & 47.95 & 2547 & 39.71 & 1.11 & 36.18 & 43.24 \\
\hline & Not used in the past month & 2170 & 59.46 & 2.33 & 52.05 & 66.86 & 2440 & 60.29 & 1.11 & 56.76 & 63.82 \\
\hline \multirow{2}{*}{ Cocaine } & Used in the past month & 692 & 13.56 & 1.06 & 10.20 & 16.92 & 603 & 10.98 & 1.95 & 4.76 & 17.20 \\
\hline & Not used in the past month & 3765 & 86.44 & 1.06 & 83.08 & 89.80 & 4384 & 89.02 & 1.95 & 82.80 & 95.24 \\
\hline \multirow{2}{*}{ Crack } & Used in the past month & 144 & 4.00 & 1.08 & 0.5431 & 7.45 & 151 & 3.634 & 1.30 & 0.00 & 7.79 \\
\hline & Not used in the past month & 4313 & 96.01 & 1.08 & 92.55 & 99.46 & 4836 & 96.37 & 1.30 & 92.21 & 100.00 \\
\hline \multirow{2}{*}{$\begin{array}{l}\text { Hallucinogens/ } \\
\text { inhalants }\end{array}$} & Used in the past month & 468 & 6.86 & 0.40 & 5.59 & 8.13 & 472 & 6.41 & 0.28 & 5.52 & 7.31 \\
\hline & Not used in the past month & 3989 & 93.14 & 0.40 & 91.87 & 94.41 & 4515 & 93.59 & 0.28 & 92.69 & 94.48 \\
\hline \multirow{2}{*}{ Stimulants } & Used in the past month & 448 & 7.85 & 0.37 & 6.66 & 9.03 & 436 & 7.47 & 0.55 & 5.72 & 9.22 \\
\hline & Not used in the past month & 4009 & 92.15 & 0.37 & 90.97 & 93.34 & 4551 & 92.53 & 0.55 & 90.78 & 94.28 \\
\hline
\end{tabular}

Note: Full notes at the end of ST 4.6. 
(ST 4.6 continued)

\begin{tabular}{|c|c|c|c|c|c|c|c|c|c|c|c|}
\hline & \multirow{4}{*}{ ore substances } & \multicolumn{10}{|c|}{ Period } \\
\hline & & \multicolumn{5}{|c|}{ (3) 2010 to 2013} & \multicolumn{5}{|c|}{ (4) 2014 to 2017} \\
\hline & & \multirow{2}{*}{$\mathbf{n}$} & \multirow{2}{*}{$\%^{a}$} & \multirow{2}{*}{ SE } & \multicolumn{2}{|c|}{$95 \% \mathrm{CI}$} & \multirow{2}{*}{$\mathbf{n}$} & \multirow{2}{*}{$\%^{a}$} & \multirow{2}{*}{ SE } & \multicolumn{2}{|c|}{$95 \% \mathrm{CI}$} \\
\hline & & & & & $\mathbf{L} \mathbf{L}$ & $\mathbf{U L}$ & & & & $\mathbf{L L}$ & $\mathbf{U L}$ \\
\hline \multirow[b]{2}{*}{ Binge alcohol } & Binge use in the past month & 1454 & 28.70 & 2.30 & 21.39 & 36.01 & 990 & 28.09 & 1.244 & 24.13 & 32.05 \\
\hline & $\begin{array}{l}\text { Never or 'no' binge use in past } \\
\text { month }\end{array}$ & 2905 & 71.30 & 2.30 & 63.99 & 78.61 & 2192 & 71.91 & 1.244 & 67.95 & 75.87 \\
\hline \multirow[b]{2}{*}{ Heavy alcohol } & Heavy use in the past month & 1215 & 23.35 & 0.88 & 20.53 & 26.16 & 738 & 21.50 & 0.392 & 20.26 & 22.75 \\
\hline & $\begin{array}{c}\text { Never or 'no' heavy use in past } \\
\text { month }\end{array}$ & 3144 & 76.65 & 0.88 & 73.84 & 79.47 & 2444 & 78.50 & 0.392 & 77.25 & 79.74 \\
\hline \multirow{2}{*}{$\begin{array}{c}\text { Tranquilizers/ } \\
\text { sedatives }\end{array}$} & Used in the past month & 963 & 21.21 & 1.06 & 17.85 & 24.57 & 712 & 20.30 & 0.483 & 18.77 & 21.84 \\
\hline & Not used in the past month & 3396 & 78.79 & 1.06 & 75.43 & 82.15 & 2470 & 79.70 & 0.483 & 78.16 & 81.23 \\
\hline \multirow{2}{*}{ Cigarettes } & Used in the past month & 2895 & 57.85 & 0.49 & 56.29 & 59.41 & 1973 & 56.26 & 1.088 & 52.79 & 59.72 \\
\hline & Not used in the past month & 1464 & 42.15 & 0.49 & 40.59 & 43.71 & 1209 & 43.74 & 1.088 & 40.28 & 47.21 \\
\hline \multirow{2}{*}{$\begin{array}{c}\text { Other tobacco } \\
\text { products }\end{array}$} & Used in the past month & 1299 & 23.23 & 0.14 & 22.79 & 23.66 & 835 & 22.12 & 0.064 & 21.92 & 22.33 \\
\hline & Not used in the past month & 3060 & 76.77 & 0.14 & 76.34 & 77.21 & 2347 & 77.88 & 0.064 & 77.67 & 78.08 \\
\hline \multirow{2}{*}{ Marijuana } & Used in the past month & 2251 & 40.68 & 1.19 & 36.88 & 44.48 & 1587 & 42.96 & 0.883 & 40.15 & 45.77 \\
\hline & Not used in the past month & 2108 & 59.32 & 1.19 & 55.52 & 63.12 & 1595 & 57.04 & 0.883 & 54.23 & 59.85 \\
\hline \multirow{2}{*}{ Cocaine } & Used in the past month & 465 & 10.13 & 0.54 & 8.40 & 11.85 & 362 & 10.31 & 0.998 & 7.14 & 13.49 \\
\hline & Not used in the past month & 3894 & 89.87 & 0.54 & 88.15 & 91.60 & 2820 & 89.69 & 0.998 & 86.51 & 92.86 \\
\hline \multirow{2}{*}{ Crack } & Used in the past month & 116 & 3.215 & 0.37 & 2.02 & 4.41 & 116 & 3.868 & 0.482 & 2.33 & 5.40 \\
\hline & Not used in the past month & 4243 & 96.79 & 0.37 & 95.59 & 97.98 & 3066 & 96.13 & 0.482 & 94.60 & 97.67 \\
\hline \multirow{2}{*}{$\begin{array}{c}\text { Hallucinogens/ } \\
\text { inhalants }\end{array}$} & Used in the past month & 416 & 7.79 & 0.62 & 5.83 & 9.76 & 246 & 5.831 & 0.656 & 3.743 & 7.92 \\
\hline & Not used in the past month & 3943 & 92.21 & 0.62 & 90.24 & 94.17 & 2936 & 94.17 & 0.656 & 92.08 & 96.26 \\
\hline \multirow{2}{*}{ Stimulants } & Used in the past month & 353 & 6.79 & 0.05 & 6.63 & 6.94 & 372 & 9.27 & 0.820 & 6.66 & 11.88 \\
\hline & Not used in the past month & 4006 & 93.21 & 0.05 & 93.06 & 93.37 & 2810 & 90.73 & 0.820 & 88.12 & 93.34 \\
\hline
\end{tabular}




\section{Table notes for ST 4.6}

Abbreviations: $\mathrm{n}=$ number of respondents, $\%=$ weighted prevalence; $\mathrm{SE}=$ standard error; $95 \% \mathrm{CI}=95 \%$ confidence interval; $\mathrm{LL}$ $=$ lower limit; $\mathrm{UL}=$ upper limit

${ }^{a}$ All weighted prevalence are based on the total number of those who reported past month use of each core substance within each period 
ST 4.7a-e | Joinpoint regression results of longitudinal changes in prevalence of significantly different past month core substance use among all PWUO, NSDUH 2002 to $2017^{\mathrm{a}, \mathrm{b}, \mathrm{c}}$

(Full table notes appear after ST 4.7e).

a) Heavy alcohol use

\begin{tabular}{|c|c|c|c|c|c|c|c|}
\hline Segment & $\begin{array}{c}\text { Lower } \\
\text { endpoint }\end{array}$ & $\begin{array}{c}\text { Upper } \\
\text { endpoint }\end{array}$ & APC & $\begin{array}{c}\text { Lower } \\
\text { CI }\end{array}$ & $\begin{array}{c}\text { Upper } \\
\text { CI }\end{array}$ & $\begin{array}{c}\text { Test } \\
\text { statistic }\end{array}$ & p-value \\
\hline $\mathbf{1}$ & 2002 & 2006 & 1.50 & -0.9 & 3.8 & 1.40 & 0.20 \\
\hline $\mathbf{2}$ & 2006 & 2017 & $-2.70^{\wedge}$ & -4.2 & -1.3 & -4.10 & $<0.001$ \\
\hline
\end{tabular}

b) Cigarette smoking

\begin{tabular}{|c|c|c|c|c|c|c|c|}
\hline Segment & $\begin{array}{c}\text { Lower } \\
\text { endpoint }\end{array}$ & $\begin{array}{c}\text { Upper } \\
\text { endpoint }\end{array}$ & APC & $\begin{array}{c}\text { Lower } \\
\text { CI }\end{array}$ & $\begin{array}{c}\text { Upper } \\
\text { CI }\end{array}$ & $\begin{array}{c}\text { Test } \\
\text { statistic }\end{array}$ & p-value \\
\hline $\mathbf{1}$ & 2002 & 2017 & 0.10 & -0.2 & 0.4 & 0.80 & 0.50 \\
\hline
\end{tabular}

c) Other tobacco products use

\begin{tabular}{|c|c|c|c|c|c|c|c|}
\hline Segment & $\begin{array}{c}\text { Lower } \\
\text { endpoint }\end{array}$ & $\begin{array}{c}\text { Upper } \\
\text { endpoint }\end{array}$ & APC & $\begin{array}{c}\text { Lower } \\
\text { CI }\end{array}$ & $\begin{array}{c}\text { Upper } \\
\text { CI }\end{array}$ & $\begin{array}{c}\text { Test } \\
\text { statistic }\end{array}$ & p-value \\
\hline $\mathbf{1}$ & 2002 & 2017 & 0.20 & -0.4 & 0.4 & 3.90 & 0.50 \\
\hline
\end{tabular}

d) Hallucinogen and/or inhalant use

\begin{tabular}{|c|c|c|c|c|c|c|c|}
\hline Segment & $\begin{array}{c}\text { Lower } \\
\text { endpoint }\end{array}$ & $\begin{array}{c}\text { Upper } \\
\text { endpoint }\end{array}$ & APC & $\begin{array}{c}\text { Lower } \\
\text { CI }\end{array}$ & $\begin{array}{c}\text { Upper } \\
\text { CI }\end{array}$ & $\begin{array}{c}\text { Test } \\
\text { statistic }\end{array}$ & p-value \\
\hline $\mathbf{1}$ & 2002 & 2017 & 0.10 & -1.0 & 1.3 & 0.30 & 0.80 \\
\hline
\end{tabular}

e) Stimulant use

\begin{tabular}{|c|c|c|c|c|c|c|c|}
\hline Segment & $\begin{array}{c}\text { Lower } \\
\text { endpoint }\end{array}$ & $\begin{array}{c}\text { Upper } \\
\text { endpoint }\end{array}$ & APC & $\begin{array}{c}\text { Lower } \\
\text { CI }\end{array}$ & $\begin{array}{c}\text { Upper } \\
\text { CI }\end{array}$ & $\begin{array}{c}\text { Test } \\
\text { statistic }\end{array}$ & p-value \\
\hline $\mathbf{1}$ & 2002 & 2004 & 5.70 & -23.8 & 46.7 & 0.40 & 0.70 \\
\hline $\mathbf{2}$ & 2004 & 2007 & -14.10 & -50.6 & 49.2 & -0.60 & 0.50 \\
\hline $\mathbf{3}$ & 2007 & 2017 & $5.4^{\wedge}$ & 2.3 & 8.5 & 4.1 & $<0.001$ \\
\hline
\end{tabular}

Abbreviations: $\mathrm{APC}=$ annual percentage change; $\mathrm{CI}=$ confidence interval

aTwo continuous variables were used to perform the joinpoint regression: 1) the weighted prevalence of Rao-Scott significant past month core substance use and 2) survey year among PWUO.

${ }^{\wedge} \mathrm{APC}$ is significantly different from zero at alpha $=0.05$. 
ST 4.8 Weighted prevalence of reported past month core substances use among the past month PPR-only use group $(n=15,897)$, NSDUH 2002 to 2017

\begin{tabular}{|c|c|c|c|c|c|c|}
\hline \multirow{2}{*}{\multicolumn{2}{|c|}{ Core Substance }} & \multirow{2}{*}{$\mathbf{n}$} & \multirow{2}{*}{$\%$} & \multirow{2}{*}{ SE } & \multicolumn{2}{|c|}{$95 \% \mathrm{CI}$} \\
\hline & & & & & $\mathbf{L L}$ & $\mathbf{U L}$ \\
\hline \multirow{2}{*}{ Binge alcohol } & $\begin{array}{l}\text { Binge use in the past } \\
\text { month }\end{array}$ & 5221 & 29.14 & 0.55 & 27.37 & 30.90 \\
\hline & $\begin{array}{l}\text { Never or 'no' binge } \\
\text { use in past month }\end{array}$ & 10676 & 70.86 & 0.55 & 69.10 & 72.63 \\
\hline \multirow{2}{*}{ Heavy alcohol } & $\begin{array}{l}\text { Heavy use in the } \\
\text { past month }\end{array}$ & 4955 & 25.53 & 0.59 & 23.66 & 27.39 \\
\hline & $\begin{array}{l}\text { Never or 'no' heavy } \\
\text { use in past month }\end{array}$ & 10942 & 74.47 & 0.59 & 72.61 & 76.34 \\
\hline \multirow{2}{*}{$\begin{array}{l}\text { Tranquilizers/ } \\
\text { sedatives }\end{array}$} & $\begin{array}{l}\text { Used in the past } \\
\text { month }\end{array}$ & 3547 & 20.43 & 0.35 & 19.31 & 21.55 \\
\hline & $\begin{array}{l}\text { Not used in the past } \\
\text { month }\end{array}$ & 12350 & 79.57 & 0.35 & 78.45 & 80.69 \\
\hline \multirow{2}{*}{ Cigarettes } & $\begin{array}{l}\text { Used in the past } \\
\text { month }\end{array}$ & 10358 & 56.66 & 0.31 & 55.68 & 57.63 \\
\hline & $\begin{array}{l}\text { Not used in the past } \\
\text { month }\end{array}$ & 5539 & 43.34 & 0.31 & 42.37 & 44.32 \\
\hline \multirow{2}{*}{$\begin{array}{l}\text { Other tobacco } \\
\text { products }\end{array}$} & $\begin{array}{l}\text { Used in the past } \\
\text { month }\end{array}$ & 4619 & 23.09 & 0.31 & 22.11 & 24.07 \\
\hline & $\begin{array}{l}\text { Not used in the past } \\
\text { month }\end{array}$ & 11278 & 76.91 & 0.31 & 75.93 & 77.89 \\
\hline \multirow{2}{*}{ Marijuana } & $\begin{array}{l}\text { Used in the past } \\
\text { month }\end{array}$ & 7998 & 39.83 & 0.54 & 38.13 & 41.54 \\
\hline & $\begin{array}{l}\text { Not used in the past } \\
\text { month }\end{array}$ & 7899 & 60.17 & 0.54 & 58.46 & 61.87 \\
\hline \multirow{2}{*}{ Cocaine } & $\begin{array}{l}\text { Used in the past } \\
\text { month }\end{array}$ & 1703 & 9.15 & 0.35 & 8.05 & 10.25 \\
\hline & $\begin{array}{l}\text { Not used in the past } \\
\text { month }\end{array}$ & 14194 & 90.85 & 0.35 & 89.75 & 91.95 \\
\hline \multirow{2}{*}{ Crack } & $\begin{array}{l}\text { Used in the past } \\
\text { month }\end{array}$ & 304 & 2.28 & 0.41 & 0.98 & 3.57 \\
\hline & $\begin{array}{l}\text { Not used in the past } \\
\text { month }\end{array}$ & 15593 & 97.72 & 0.41 & 96.43 & 99.02 \\
\hline \multirow{2}{*}{$\begin{array}{l}\text { Hallucinogens/ } \\
\text { inhalants }\end{array}$} & $\begin{array}{l}\text { Used in the past } \\
\text { month }\end{array}$ & 1441 & 6.37 & 0.20 & 5.72 & 7.01 \\
\hline & $\begin{array}{l}\text { Not used in the past } \\
\text { month }\end{array}$ & 14456 & 93.63 & 0.20 & 92.99 & 94.28 \\
\hline \multirow{2}{*}{ Stimulants } & $\begin{array}{l}\text { Used in the past } \\
\text { month }\end{array}$ & 1483 & 7.63 & 0.23 & 6.91 & 8.35 \\
\hline & $\begin{array}{l}\text { Not used in the past } \\
\text { month }\end{array}$ & 14414 & 92.37 & 0.23 & 91.65 & 93.09 \\
\hline
\end{tabular}

Abbreviations: $\mathrm{n}=$ number of respondents, $\%=$ weighted prevalence; $\mathrm{SE}=$ standard error; $95 \%$ $\mathrm{CI}=95 \%$ confidence interval; $\mathrm{LL}=$ lower limit; $\mathrm{UL}=$ upper limit 
ST 4.9 Weighted prevalence of reported past month core substances use among the past month heroin-only use group $(\mathrm{n}=553)$, NSDUH 2002 to 2017

\begin{tabular}{|c|c|c|c|c|c|c|}
\hline \multirow{2}{*}{\multicolumn{2}{|c|}{ Core Substance }} & \multirow{2}{*}{$\mathbf{n}$} & \multirow{2}{*}{$\%$} & \multirow{2}{*}{ SE } & \multicolumn{2}{|c|}{$95 \% \mathrm{CI}$} \\
\hline & & & & & $\mathbf{L} \mathbf{L}$ & $\mathbf{U L}$ \\
\hline \multirow{2}{*}{ Binge alcohol } & $\begin{array}{l}\text { Binge use in the past } \\
\text { month }\end{array}$ & 138 & 23.37 & 1.33 & 19.13 & 27.61 \\
\hline & $\begin{array}{l}\text { Never or 'no' binge } \\
\text { use in past month }\end{array}$ & 415 & 76.63 & 1.33 & 72.39 & 80.87 \\
\hline \multirow{2}{*}{ Heavy alcohol } & $\begin{array}{l}\text { Heavy use in the past } \\
\text { month }\end{array}$ & 99 & 16.74 & 2.35 & 9.26 & 24.21 \\
\hline & $\begin{array}{l}\text { Never or 'no' heavy } \\
\text { use in past month }\end{array}$ & 454 & 83.26 & 2.35 & 75.79 & 90.74 \\
\hline \multirow{2}{*}{$\begin{array}{l}\text { Tranquilizers/ } \\
\text { sedatives }\end{array}$} & $\begin{array}{l}\text { Used in the past } \\
\text { month }\end{array}$ & 79 & 13.39 & 0.91 & 10.48 & 16.29 \\
\hline & $\begin{array}{l}\text { Not used in the past } \\
\text { month }\end{array}$ & 474 & 86.61 & 0.91 & 83.71 & 89.52 \\
\hline \multirow{2}{*}{ Cigarettes } & $\begin{array}{l}\text { Used in the past } \\
\text { month }\end{array}$ & 495 & 88.63 & 1.06 & 85.25 & 92.01 \\
\hline & $\begin{array}{l}\text { Not used in the past } \\
\text { month }\end{array}$ & 58 & 11.37 & 1.06 & 7.99 & 14.75 \\
\hline \multirow{2}{*}{$\begin{array}{l}\text { Other tobacco } \\
\text { products }\end{array}$} & $\begin{array}{l}\text { Used in the past } \\
\text { month }\end{array}$ & 148 & 22.75 & 2.35 & 15.27 & 30.22 \\
\hline & $\begin{array}{l}\text { Not used in the past } \\
\text { month }\end{array}$ & 405 & 77.25 & 2.35 & 69.78 & 84.73 \\
\hline \multirow{2}{*}{ Marijuana } & $\begin{array}{l}\text { Used in the past } \\
\text { month }\end{array}$ & 303 & 48.13 & 4.84 & 32.71 & 63.55 \\
\hline & $\begin{array}{l}\text { Not used in the past } \\
\text { month }\end{array}$ & 250 & 51.87 & 4.84 & 36.45 & 67.29 \\
\hline \multirow{2}{*}{ Cocaine } & $\begin{array}{l}\text { Used in the past } \\
\text { month }\end{array}$ & 189 & 35.36 & 1.50 & 30.57 & 40.15 \\
\hline & $\begin{array}{l}\text { Not used in the past } \\
\text { month }\end{array}$ & 364 & 64.64 & 1.50 & 59.85 & 69.43 \\
\hline \multirow{2}{*}{ Crack } & $\begin{array}{l}\text { Used in the past } \\
\text { month }\end{array}$ & 102 & 20.00 & 2.45 & 12.19 & 27.81 \\
\hline & $\begin{array}{l}\text { Not used in the past } \\
\text { month }\end{array}$ & 451 & 80.00 & 2.45 & 72.19 & 87.81 \\
\hline \multirow{2}{*}{$\begin{array}{l}\text { Hallucinogens/ } \\
\text { inhalants }\end{array}$} & $\begin{array}{l}\text { Used in the past } \\
\text { month }\end{array}$ & 58 & 8.66 & 1.45 & 4.04 & 13.28 \\
\hline & $\begin{array}{l}\text { Not used in the past } \\
\text { month }\end{array}$ & 495 & 91.34 & 1.45 & 86.72 & 95.96 \\
\hline \multirow{2}{*}{ Stimulants } & $\begin{array}{l}\text { Used in the past } \\
\text { month }\end{array}$ & 26 & 3.96 & 1.50 & 0.00 & 8.74 \\
\hline & $\begin{array}{l}\text { Not used in the past } \\
\text { month }\end{array}$ & 527 & 96.04 & 1.50 & 91.26 & 100.00 \\
\hline
\end{tabular}

Abbreviations: $\mathrm{n}=$ number of respondents, $\%=$ weighted prevalence; $\mathrm{SE}=$ standard error; $95 \%$ $\mathrm{CI}=95 \%$ confidence interval; $\mathrm{LL}=$ lower limit; $\mathrm{UL}=$ upper limit 
ST 4.10 | Weighted prevalence of reported past month core substances use among the past month combination opioid use group $(n=535)$, NSDUH 2002 to 2017

\begin{tabular}{|c|c|c|c|c|c|c|}
\hline \multicolumn{2}{|c|}{ Core Substance } & \multirow[t]{2}{*}{$\mathbf{n}$} & \multirow[t]{2}{*}{$\%$} & \multirow[t]{2}{*}{ SE } & \multicolumn{2}{|c|}{$95 \% \mathrm{CI}$} \\
\hline & & & & & $\mathbf{L L}$ & $\mathbf{U L}$ \\
\hline \multirow[t]{2}{*}{ Binge alcohol } & $\begin{array}{l}\text { Binge use in the past } \\
\text { month }\end{array}$ & 164 & 24.95 & 2.86 & 15.85 & 34.06 \\
\hline & $\begin{array}{l}\text { Never or 'no' binge } \\
\text { use in past month }\end{array}$ & 371 & 75.05 & 2.86 & 65.94 & 84.15 \\
\hline \multirow[t]{2}{*}{ Heavy alcohol } & $\begin{array}{l}\text { Heavy use in the past } \\
\text { month }\end{array}$ & 157 & 29.27 & 5.37 & 12.17 & 46.37 \\
\hline & $\begin{array}{l}\text { Never or 'no' heavy } \\
\text { use in past month }\end{array}$ & 378 & 70.73 & 5.37 & 53.63 & 87.83 \\
\hline \multirow[t]{2}{*}{$\begin{array}{l}\text { Tranquilizers/ } \\
\text { sedatives }\end{array}$} & $\begin{array}{l}\text { Used in the past } \\
\text { month }\end{array}$ & 234 & 44.48 & 2.88 & 35.32 & 53.65 \\
\hline & $\begin{array}{l}\text { Not used in the past } \\
\text { month }\end{array}$ & 301 & 55.52 & 2.88 & 46.35 & 64.68 \\
\hline \multirow[t]{2}{*}{ Cigarettes } & $\begin{array}{l}\text { Used in the past } \\
\text { month }\end{array}$ & 493 & 87.38 & 2.02 & 80.96 & 93.80 \\
\hline & $\begin{array}{l}\text { Not used in the past } \\
\text { month }\end{array}$ & 42 & 12.62 & 2.02 & 6.21 & 19.04 \\
\hline \multirow[t]{2}{*}{$\begin{array}{l}\text { Other tobacco } \\
\text { products }\end{array}$} & $\begin{array}{l}\text { Used in the past } \\
\text { month }\end{array}$ & 204 & 38.91 & 0.70 & 36.67 & 41.14 \\
\hline & $\begin{array}{l}\text { Not used in the past } \\
\text { month }\end{array}$ & 331 & 61.09 & 0.70 & 58.86 & 63.33 \\
\hline \multirow[t]{2}{*}{ Marijuana } & $\begin{array}{l}\text { Used in the past } \\
\text { month }\end{array}$ & 371 & 64.75 & 2.41 & 57.08 & 72.43 \\
\hline & $\begin{array}{l}\text { Not used in the past } \\
\text { month }\end{array}$ & 164 & 35.25 & 2.41 & 27.57 & 42.92 \\
\hline \multirow[t]{2}{*}{ Cocaine } & $\begin{array}{l}\text { Used in the past } \\
\text { month }\end{array}$ & 230 & 45.96 & 2.88 & 36.81 & 55.11 \\
\hline & $\begin{array}{l}\text { Not used in the past } \\
\text { month }\end{array}$ & 305 & 54.04 & 2.88 & 44.89 & 63.19 \\
\hline \multirow[t]{2}{*}{ Crack } & $\begin{array}{l}\text { Used in the past } \\
\text { month }\end{array}$ & 121 & 26.80 & 1.14 & 23.18 & 30.42 \\
\hline & $\begin{array}{l}\text { Not used in the past } \\
\text { month }\end{array}$ & 414 & 73.20 & 1.14 & 69.58 & 76.82 \\
\hline \multirow[t]{2}{*}{$\begin{array}{l}\text { Hallucinogens/ } \\
\text { inhalants }\end{array}$} & $\begin{array}{l}\text { Used in the past } \\
\text { month }\end{array}$ & 103 & 16.45 & 2.99 & 6.93 & 25.97 \\
\hline & $\begin{array}{l}\text { Not used in the past } \\
\text { month }\end{array}$ & 432 & 83.55 & 2.99 & 74.03 & 93.07 \\
\hline \multirow[t]{2}{*}{ Stimulants } & $\begin{array}{l}\text { Used in the past } \\
\text { month }\end{array}$ & 100 & 16.75 & 2.20 & 9.76 & 23.75 \\
\hline & $\begin{array}{l}\text { Not used in the past } \\
\text { month }\end{array}$ & 435 & 83.25 & 2.20 & 76.25 & 90.24 \\
\hline
\end{tabular}

Abbreviations: $\mathrm{n}=$ number of respondents, $\%=$ weighted prevalence; $\mathrm{SE}=$ standard error; $95 \%$ $\mathrm{CI}=95 \%$ confidence interval; $\mathrm{LL}=$ lower limit; $\mathrm{UL}=$ upper limit 
ST 4.11 | Weighted prevalence of reported substance use for past month PPR-only use group by period ( $\mathrm{n}=15,897)$, NSDUH 2002 to 2017

\begin{tabular}{|c|c|c|c|c|c|c|c|c|c|c|c|}
\hline \multirow{4}{*}{\multicolumn{2}{|c|}{ Core substances }} & \multicolumn{10}{|c|}{ Period } \\
\hline & & \multicolumn{5}{|c|}{ (1) 2002 to 2005} & \multicolumn{5}{|c|}{ (2) 2006 to 2009} \\
\hline & & \multirow{2}{*}{$\mathbf{n}$} & \multirow{2}{*}{$\%^{a}$} & \multirow{2}{*}{ SE } & \multicolumn{2}{|c|}{$95 \% \mathrm{CI}$} & \multirow{2}{*}{$\mathbf{n}$} & \multirow{2}{*}{$\%^{a}$} & \multirow{2}{*}{ SE } & \multicolumn{2}{|c|}{$95 \% \mathrm{CI}$} \\
\hline & & & & & $\mathbf{L} \mathbf{L}$ & UL & & & & $\mathbf{L L}$ & UL \\
\hline \multirow[b]{2}{*}{ Binge alcohol } & Binge use in the past month & 1379 & 28.06 & 1.76 & 22.66 & 33.86 & 1598 & 27.96 & 0.15 & 30.15 & 31.11 \\
\hline & $\begin{array}{l}\text { Never or 'no' binge use in } \\
\text { past month }\end{array}$ & 2927 & 71.74 & 1.76 & 66.14 & 77.34 & 3172 & 69.37 & 0.15 & 68.89 & 69.85 \\
\hline \multirow[b]{2}{*}{ Heavy alcohol } & Heavy use in the past month & 1526 & 28.25 & 1.71 & 22.81 & 33.68 & 1610 & 27.50 & 0.21 & 26.82 & 28.17 \\
\hline & $\begin{array}{l}\text { Never or 'no' heavy use in } \\
\text { past month }\end{array}$ & 2780 & 71.75 & 1.71 & 66.32 & 77.19 & 3160 & 72.50 & 0.21 & 71.83 & 73.18 \\
\hline \multirow{2}{*}{$\begin{array}{l}\text { Tranquilizers/ } \\
\text { sedatives }\end{array}$} & Used in the past month & 977 & 21.68 & 0.48 & 20.16 & 23.21 & 1101 & 20.56 & 0.04 & 20.44 & 20.67 \\
\hline & Not used in the past month & 3329 & 78.32 & 0.48 & 76.79 & 79.84 & 3669 & 79.44 & 0.04 & 79.33 & 79.56 \\
\hline \multirow{2}{*}{ Cigarettes } & Used in the past month & 2905 & 59.09 & 0.94 & 56.10 & 62.08 & 3239 & 58.96 & 0.39 & 57.72 & 60.20 \\
\hline & Not used in the past month & 1401 & 40.91 & 0.94 & 37.92 & 43.90 & 1531 & 41.04 & 0.39 & 39.80 & 42.28 \\
\hline \multirow{2}{*}{$\begin{array}{l}\text { Other tobacco } \\
\text { products }\end{array}$} & Used in the past month & 1267 & 23.81 & 1.03 & 20.52 & 27.09 & 1448 & 24.27 & 0.07 & 24.04 & 24.49 \\
\hline & Not used in the past month & 3039 & 76.19 & 1.03 & 72.91 & 79.48 & 3322 & 75.73 & 0.07 & 75.51 & 75.96 \\
\hline \multirow{2}{*}{ Marijuana } & Used in the past month & 2193 & 40.11 & 2.10 & 33.42 & 46.80 & 2418 & 39.41 & 1.75 & 33.83 & 44.99 \\
\hline & Not used in the past month & 2113 & 35.98 & 1.32 & 31.79 & 40.18 & 2352 & 60.59 & 1.75 & 55.01 & 66.17 \\
\hline \multirow{2}{*}{ Cocaine } & Used in the past month & 623 & 12.44 & 1.07 & 9.05 & 15.83 & 511 & 9.21 & 2.07 & 2.62 & 15.79 \\
\hline & Not used in the past month & 3683 & 87.56 & 1.07 & 84.17 & 90.95 & 4259 & 90.80 & 2.07 & 84.21 & 97.38 \\
\hline \multirow{2}{*}{ Crack } & Used in the past month & 113 & 3.42 & 0.81 & 0.83 & 6.01 & 97 & 2.43 & 1.45 & 0.00 & 7.05 \\
\hline & Not used in the past month & 4193 & 96.58 & 0.81 & 93.99 & 99.17 & 4673 & 97.57 & 1.45 & 92.95 & 100.00 \\
\hline \multirow{2}{*}{$\begin{array}{c}\text { Hallucinogens/ } \\
\text { inhalants }\end{array}$} & Used in the past month & 436 & 6.63 & 0.44 & 5.23 & 8.03 & 435 & 6.23 & 0.31 & 5.24 & 7.21 \\
\hline & Not used in the past month & 3870 & 93.37 & 0.44 & 91.97 & 94.77 & 4335 & 93.77 & 0.31 & 92.79 & 94.76 \\
\hline \multirow{2}{*}{ Stimulants } & Used in the past month & 433 & 7.98 & 0.40 & 6.72 & 9.24 & 415 & 7.58 & 0.52 & 5.93 & 9.23 \\
\hline & Not used in the past month & 3873 & 92.02 & 0.40 & 90.76 & 93.28 & 4355 & 92.42 & 0.52 & 90.78 & 94.07 \\
\hline
\end{tabular}

Note: Full notes at the end of the ST 4.11. 
(ST 4.11 continued)

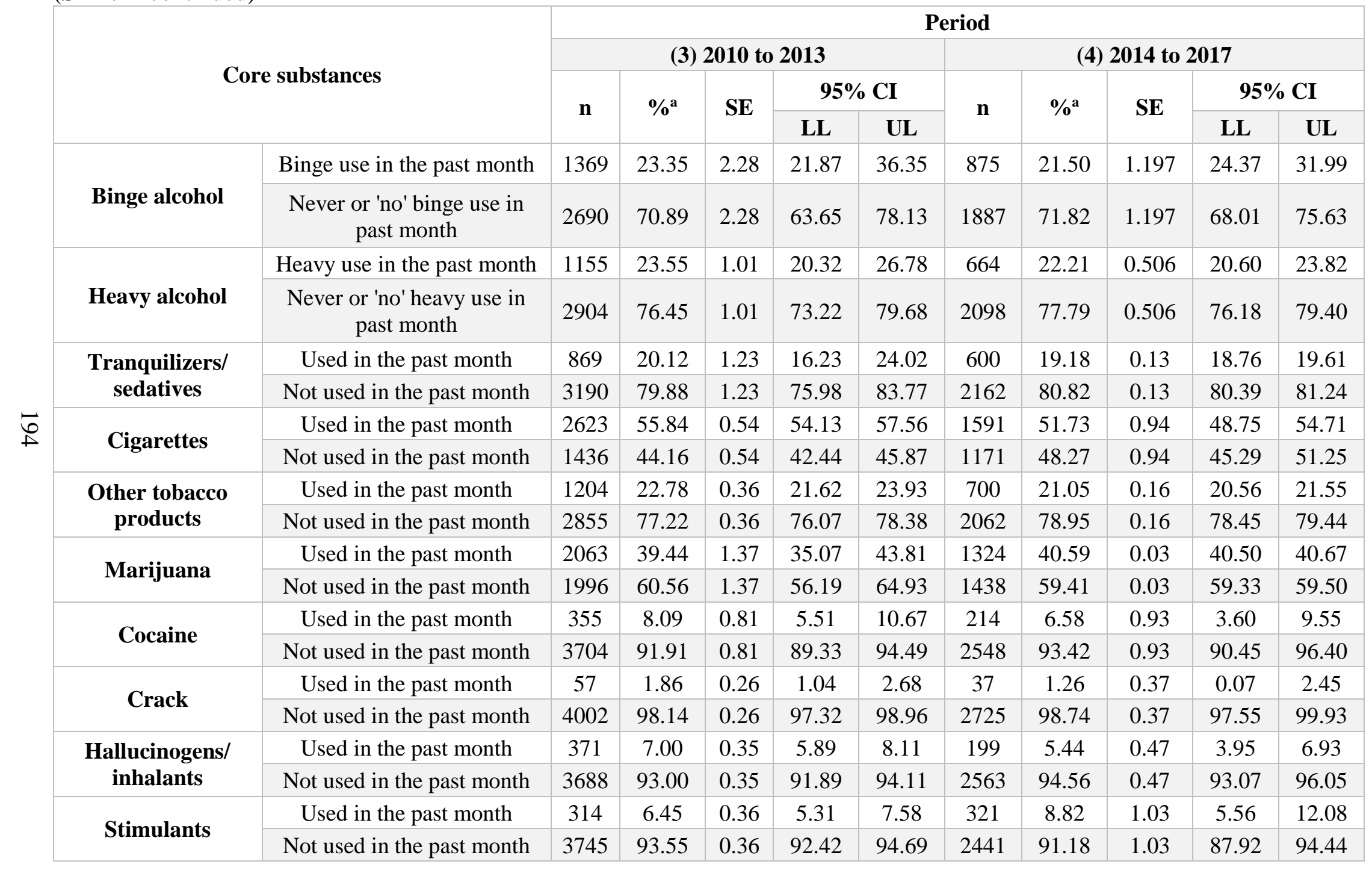




\section{Table notes for ST 4.11}

Abbreviations: $\mathrm{n}=$ number of respondents, $\%=$ weighted prevalence; $\mathrm{SE}=$ standard error; $95 \% \mathrm{CI}=95 \%$ confidence interval; $\mathrm{LL}$ = lower limit; UL = upper limit

${ }^{a}$ All weighted prevalence are based on the total number of those who reported past month use of each core substance within each period 
ST 4.12a-c $\mid$ Joinpoint regression results of longitudinal changes in prevalence of significantly different past month core substance use among past month PPR-only use group, NSDUH 2002 to $2017^{\mathrm{a}, \mathrm{b}, \mathrm{c}}$

\section{a) Heavy alcohol use}

\begin{tabular}{|c|c|c|c|c|c|c|c|}
\hline Segment & $\begin{array}{c}\text { Lower } \\
\text { endpoint }\end{array}$ & $\begin{array}{c}\text { Upper } \\
\text { endpoint }\end{array}$ & APC & $\begin{array}{c}\text { Lower } \\
\text { CI }\end{array}$ & $\begin{array}{c}\text { Upper } \\
\text { CI }\end{array}$ & $\begin{array}{c}\text { Test } \\
\text { statistic }\end{array}$ & p-value \\
\hline $\mathbf{1}$ & 2002 & 2007 & 0.60 & -1.1 & 2.3 & 0.80 & 0.40 \\
\hline $\mathbf{2}$ & 2007 & 2017 & $-2.70^{\wedge}$ & -3.9 & -1.5 & -4.80 & $<0.001$ \\
\hline
\end{tabular}

b) Cigarette smoking

\begin{tabular}{|c|c|c|c|c|c|c|c|}
\hline Segment & $\begin{array}{c}\text { Lower } \\
\text { endpoint }\end{array}$ & $\begin{array}{c}\text { Upper } \\
\text { endpoint }\end{array}$ & APC & $\begin{array}{c}\text { Lower } \\
\text { CI }\end{array}$ & $\begin{array}{c}\text { Upper } \\
\text { CI }\end{array}$ & $\begin{array}{c}\text { Test } \\
\text { statistic }\end{array}$ & p-value \\
\hline $\mathbf{1}$ & 2002 & 2017 & $-0.90^{\wedge}$ & -1.8 & -0.1 & -2.40 & $<0.001$ \\
\hline
\end{tabular}

c) Other tobacco products use

\begin{tabular}{|c|c|c|c|c|c|c|c|}
\hline Segment & $\begin{array}{c}\text { Lower } \\
\text { endpoint }\end{array}$ & $\begin{array}{c}\text { Upper } \\
\text { endpoint }\end{array}$ & APC & $\begin{array}{c}\text { Lower } \\
\text { CI }\end{array}$ & $\begin{array}{c}\text { Upper } \\
\text { CI }\end{array}$ & $\begin{array}{c}\text { Test } \\
\text { statistic }\end{array}$ & p-value \\
\hline $\mathbf{1}$ & 2002 & 2017 & $0.40^{\wedge}$ & 0.0 & 0.7 & 2.20 & $<0.001$ \\
\hline
\end{tabular}

\section{d) Hallucinogen and/or inhalant use}

\begin{tabular}{|c|c|c|c|c|c|c|c|}
\hline Segment & $\begin{array}{c}\text { Lower } \\
\text { endpoint }\end{array}$ & $\begin{array}{c}\text { Upper } \\
\text { endpoint }\end{array}$ & APC & $\begin{array}{c}\text { Lower } \\
\text { CI }\end{array}$ & $\begin{array}{c}\text { Upper } \\
\text { CI }\end{array}$ & $\begin{array}{c}\text { Test } \\
\text { statistic }\end{array}$ & p-value \\
\hline $\mathbf{1}$ & 2002 & 2017 & -1.10 & -2.5 & 0.2 & -1.80 & 0.10 \\
\hline
\end{tabular}

Abbreviations: $\mathrm{APC}=$ annual percentage change; $\mathrm{CI}=$ confidence interval

${ }^{a}$ Two continuous variables were used to perform the joinpoint regression: 1) the weighted prevalence of Rao-Scott significant past month core substance use and 2) survey year among past month PPR-only use group.

${ }^{\wedge} \mathrm{APC}$ is significantly different from zero at alpha $=0.05$. 
ST 4.13 Weighted prevalence of reported substance use for past month heroin-only use group by period ( $\mathrm{n}=553)$, NSDUH 2002 to 2017

\begin{tabular}{|c|c|c|c|c|c|c|c|c|c|c|c|}
\hline \multirow{4}{*}{\multicolumn{2}{|c|}{ Core substances }} & \multicolumn{10}{|c|}{ Period } \\
\hline & & \multicolumn{5}{|c|}{ (1) 2002 to 2005} & \multicolumn{5}{|c|}{ (2) 2006 to 2009} \\
\hline & & \multirow[b]{3}{*}{19} & \multirow{3}{*}{$\begin{array}{c}\%^{\mathbf{a}} \\
22.86\end{array}$} & \multirow{3}{*}{$\begin{array}{l}\mathbf{S E} \\
2.35\end{array}$} & \multicolumn{2}{|c|}{$95 \%$ CI } & \multirow{3}{*}{$\begin{array}{l}\mathbf{n} \\
22\end{array}$} & \multirow{3}{*}{$\begin{array}{c}\%^{\mathrm{a}} \\
18.50\end{array}$} & \multirow{3}{*}{$\begin{array}{c}\text { SE } \\
7.50\end{array}$} & \multicolumn{2}{|c|}{$95 \% \mathrm{CI}$} \\
\hline & & & & & $\mathbf{L L}$ & $\mathbf{U L}$ & & & & $\mathbf{L L}$ & $\mathbf{U L}$ \\
\hline \multirow[b]{2}{*}{ Binge alcohol } & Binge use in the past month & & & & 15.37 & 30.35 & & & & 0.00 & 42.36 \\
\hline & $\begin{array}{l}\text { Never or 'no' binge use in } \\
\text { past month }\end{array}$ & 61 & 77.14 & 2.35 & 69.65 & 84.63 & 66 & 81.50 & 7.50 & 57.64 & 100.00 \\
\hline \multirow[b]{2}{*}{ Heavy alcohol } & Heavy use in the past month & 17 & 14.21 & 3.19 & 4.06 & 24.35 & 27 & 27.57 & 9.63 & 0.00 & 58.21 \\
\hline & $\begin{array}{c}\text { Never or 'no' heavy use in } \\
\text { past month }\end{array}$ & 63 & 85.80 & 3.19 & 75.65 & 95.94 & 61 & 72.43 & 9.63 & 41.79 & 100.00 \\
\hline \multirow{2}{*}{$\begin{array}{l}\text { Tranquilizers/ } \\
\text { sedatives }\end{array}$} & Used in the past month & 8 & 8.36 & 3.54 & 0.00 & 19.63 & 13 & 10.30 & 5.94 & 0.00 & 29.21 \\
\hline & Not used in the past month & 72 & 91.64 & 3.54 & 80.37 & 100.00 & 75 & 89.70 & 5.94 & 70.79 & 100.00 \\
\hline \multirow{2}{*}{ Cigarettes } & Used in the past month & 69 & 86.70 & 2.53 & 78.64 & 94.76 & 82 & 95.43 & 2.52 & 87.42 & 100.00 \\
\hline & Not used in the past month & 11 & 13.30 & 2.53 & 5.24 & 21.36 & 6 & 4.57 & 2.52 & 0.00 & 12.58 \\
\hline \multirow{2}{*}{$\begin{array}{l}\text { Other tobacco } \\
\text { products }\end{array}$} & Used in the past month & 19 & 20.27 & 3.24 & 9.95 & 30.58 & 23 & 25.50 & 12.26 & 0.00 & 64.52 \\
\hline & Not used in the past month & 61 & 79.73 & 3.24 & 69.42 & 90.05 & 65 & 74.50 & 12.26 & 35.48 & 100.00 \\
\hline \multirow{2}{*}{ Marijuana } & Used in the past month & 40 & 39.94 & 14.25 & 0.00 & 85.27 & 45 & 33.39 & 11.05 & 0.00 & 68.57 \\
\hline & Not used in the past month & 40 & 60.06 & 14.25 & 14.73 & 100.00 & 43 & 66.61 & 11.05 & 31.43 & 100.00 \\
\hline \multirow{2}{*}{ Cocaine } & Used in the past month & 34 & 42.32 & 6.18 & 22.65 & 61.99 & 34 & 38.29 & 8.72 & 10.52 & 66.05 \\
\hline & Not used in the past month & 46 & 57.68 & 6.18 & 38.01 & 77.35 & 54 & 61.71 & 8.72 & 33.95 & 89.48 \\
\hline \multirow{2}{*}{ Crack } & Used in the past month & 14 & 18.47 & 13.26 & 0.00 & 60.66 & 21 & 22.11 & 1.75 & 16.54 & 27.69 \\
\hline & Not used in the past month & 66 & 81.53 & 13.26 & 39.34 & 100.00 & 67 & 77.89 & 1.75 & 72.31 & 83.46 \\
\hline \multirow{2}{*}{$\begin{array}{l}\text { Hallucinogens/ } \\
\text { inhalants }\end{array}$} & Used in the past month & 11 & 5.28 & 1.87 & 0.00 & 11.22 & 6 & 5.85 & 2.70 & 0.00 & 14.43 \\
\hline & Not used in the past month & 69 & 94.72 & 1.87 & 88.78 & 100.00 & 82 & 94.15 & 2.70 & 85.57 & 100.00 \\
\hline \multirow{2}{*}{ Stimulants } & Used in the past month & 3 & 1.09 & 0.46 & 0.00 & 2.56 & 3 & 6.09 & 2.76 & 0.00 & 14.86 \\
\hline & Not used in the past month & 77 & 98.91 & 0.46 & 97.44 & 100.00 & 85 & 93.91 & 2.76 & 85.14 & 100.00 \\
\hline
\end{tabular}

Note: Full notes at the end of ST 4.13. 
(ST 4.13 continued)

\begin{tabular}{|c|c|c|c|c|c|c|c|c|c|c|c|}
\hline \multirow{4}{*}{\multicolumn{2}{|c|}{ Core substances }} & \multicolumn{10}{|c|}{ Period } \\
\hline & & \multicolumn{5}{|c|}{ (3) 2010 to 2013} & \multicolumn{5}{|c|}{ (4) 2014 to 2017} \\
\hline & & \multirow{2}{*}{$\mathbf{n}$} & \multirow{2}{*}{$\%^{a}$} & \multirow{2}{*}{ SE } & \multicolumn{2}{|c|}{$95 \% \mathrm{CI}$} & \multirow{2}{*}{$\mathbf{n}$} & \multirow{2}{*}{$\%^{a}$} & \multirow{2}{*}{$\mathbf{S E}$} & \multicolumn{2}{|c|}{$95 \% \mathrm{CI}$} \\
\hline & & & & & $\mathbf{L L}$ & $\mathbf{U L}$ & & & & $\mathbf{L L}$ & $\mathbf{U L}$ \\
\hline \multirow[b]{2}{*}{ Binge alcohol } & Binge use in the past month & 36 & 19.05 & 1.91 & 12.99 & 25.11 & 61 & 27.26 & 0.003 & 27.25 & 27.27 \\
\hline & $\begin{array}{l}\text { Never or 'no' binge use in } \\
\text { past month }\end{array}$ & 108 & 80.95 & 1.91 & 74.89 & 87.02 & 180 & 72.74 & 0.003 & 72.73 & 72.75 \\
\hline \multirow[b]{2}{*}{ Heavy alcohol } & Heavy use in the past month & 21 & 17.68 & 2.64 & 9.28 & 26.08 & 34 & 13.04 & 1.588 & 7.98 & 18.09 \\
\hline & $\begin{array}{l}\text { Never or 'no' heavy use in } \\
\text { past month }\end{array}$ & 123 & 82.32 & 2.64 & 73.92 & 90.72 & 207 & 86.96 & 1.588 & 81.91 & 92.02 \\
\hline \multirow{2}{*}{$\begin{array}{l}\text { Tranquilizers/ } \\
\text { sedatives }\end{array}$} & Used in the past month & 17 & 12.87 & 0.40 & 11.59 & 14.15 & 41 & 16.37 & 1.09 & 12.90 & 19.84 \\
\hline & Not used in the past month & 127 & 87.13 & 0.40 & 85.85 & 88.42 & 200 & 83.63 & 1.09 & 80.16 & 87.10 \\
\hline \multirow{2}{*}{ Cigarettes } & Used in the past month & 123 & 85.75 & 1.81 & 80.00 & 91.50 & 221 & 87.93 & 1.52 & 83.10 & 92.77 \\
\hline & Not used in the past month & 21 & 14.25 & 1.81 & 8.50 & 20.00 & 20 & 12.07 & 1.52 & 7.23 & 16.90 \\
\hline \multirow{2}{*}{$\begin{array}{l}\text { Other tobacco } \\
\text { products }\end{array}$} & Used in the past month & 36 & 15.75 & 0.48 & 14.21 & 17.29 & 70 & 25.56 & 2.99 & 16.03 & 35.08 \\
\hline & Not used in the past month & 108 & 84.25 & 0.48 & 82.71 & 85.79 & 171 & 74.44 & 2.99 & 64.92 & 83.97 \\
\hline \multirow{2}{*}{ Marijuana } & Used in the past month & 76 & 50.25 & 0.03 & 50.16 & 50.35 & 142 & 55.35 & 7.77 & 30.63 & 80.07 \\
\hline & Not used in the past month & 68 & 49.75 & 0.03 & 49.65 & 49.84 & 99 & 44.65 & 7.77 & 19.93 & 69.37 \\
\hline \multirow{2}{*}{ Cocaine } & Used in the past month & 41 & 32.87 & 8.91 & 4.51 & 61.23 & 80 & 33.13 & 2.36 & 25.63 & 40.63 \\
\hline & Not used in the past month & 103 & 67.13 & 8.91 & 38.77 & 95.49 & 161 & 66.87 & 2.36 & 59.37 & 74.37 \\
\hline \multirow{2}{*}{ Crack } & Used in the past month & 22 & 18.15 & 8.94 & 0.00 & 46.59 & 45 & 20.50 & 2.26 & 13.31 & 27.69 \\
\hline & Not used in the past month & 122 & 81.85 & 8.94 & 53.41 & 100.00 & 196 & 79.50 & 2.26 & 72.31 & 86.69 \\
\hline \multirow{2}{*}{$\begin{array}{l}\text { Hallucinogens/ } \\
\text { inhalants }\end{array}$} & Used in the past month & 15 & 12.33 & 1.04 & 9.01 & 15.66 & 26 & 9.19 & 2.79 & 0.32 & 18.05 \\
\hline & Not used in the past month & 129 & 87.67 & 1.04 & 84.34 & 90.99 & 215 & 90.81 & 2.79 & 81.95 & 99.68 \\
\hline \multirow{2}{*}{ Stimulants } & Used in the past month & 10 & 4.36 & 1.98 & 0.00 & 10.65 & 10 & 3.89 & 2.53 & 0.00 & 11.94 \\
\hline & Not used in the past month & 134 & 95.64 & 1.98 & 89.35 & 100.00 & 231 & 96.11 & 2.53 & 88.06 & 100.00 \\
\hline
\end{tabular}




\section{Table notes for ST 4.13}

Abbreviations: $\mathrm{n}=$ number of respondents, $\%=$ weighted prevalence; $\mathrm{SE}=$ standard error; $95 \% \mathrm{CI}=95 \%$ confidence interval; $\mathrm{LL}$ $=$ lower limit; $\mathrm{UL}=$ upper limit

${ }^{a}$ All weighted prevalence are based on the total number of those who reported past month use of each core substance within each period 
ST 4.14a-b | Joinpoint regression results of longitudinal changes in prevalence of significantly different past month core substance use among past month heroin-only use group, NSDUH 2002 to $2017^{\mathrm{a}, \mathrm{b}, \mathrm{c}}$

\section{a) Heavy alcohol use}

\begin{tabular}{|c|c|c|c|c|c|c|}
\hline Segment & $\begin{array}{c}\text { Lower } \\
\text { endpoint }\end{array}$ & $\begin{array}{c}\text { Upper } \\
\text { endpoint }\end{array}$ & Slope & SE & $\begin{array}{c}\text { Test } \\
\text { statistic }\end{array}$ & p-value \\
\hline $\mathbf{1}$ & 2002 & 2017 & -0.66 & 0.4 & -1.79 & 0.10 \\
\hline
\end{tabular}

\section{b) Cigarette smoking}

\begin{tabular}{|c|c|c|c|c|c|c|c|}
\hline Segment & $\begin{array}{c}\text { Lower } \\
\text { endpoint }\end{array}$ & $\begin{array}{c}\text { Upper } \\
\text { endpoint }\end{array}$ & APC & $\begin{array}{c}\text { Lower } \\
\text { CI }\end{array}$ & $\begin{array}{c}\text { Upper } \\
\text { CI }\end{array}$ & $\begin{array}{c}\text { Test } \\
\text { statistic }\end{array}$ & p-value \\
\hline $\mathbf{1}$ & 2002 & 2008 & 2.60 & -1.4 & 6.9 & 1.40 & 0.20 \\
\hline $\mathbf{2}$ & 2008 & 2017 & $-2.30^{\wedge}$ & -3.6 & -0.9 & -3.7 & $<0.001$ \\
\hline
\end{tabular}

Abbreviations: $\mathrm{APC}=$ annual percentage change $\mathrm{CI}=$ confidence interval aTwo continuous variables were used to perform the joinpoint regression: 1) the weighted prevalence of Rao-Scott significant past month core substance use and 2) survey year among past month heroin-only use group.

${ }^{\wedge} \mathrm{APC}$ is significantly different from zero at alpha $=0.05$. 
ST 4.15 Weighted prevalence of reported substance use for past month combination opioid use group by period $(\mathrm{n}=535)$, NSDUH 2002 to 2017

\begin{tabular}{|c|c|c|c|c|c|c|c|c|c|c|c|}
\hline \multirow{4}{*}{\multicolumn{2}{|c|}{ Core substances }} & \multicolumn{10}{|c|}{ Period } \\
\hline & & \multicolumn{5}{|c|}{ (1) 2002 to 2005} & \multicolumn{5}{|c|}{ (2) 2006 to 2009} \\
\hline & & \multirow{3}{*}{$\begin{array}{l}\mathbf{n} \\
25\end{array}$} & \multirow{3}{*}{$\begin{array}{c}\%^{\mathbf{a}} \\
27.81\end{array}$} & \multirow{3}{*}{$\begin{array}{c}\text { SE } \\
5.05\end{array}$} & \multicolumn{2}{|c|}{$95 \% \mathrm{CI}$} & \multirow{3}{*}{$\begin{array}{l}\mathbf{n} \\
36\end{array}$} & \multirow{3}{*}{$\begin{array}{c}\%^{\mathbf{a}} \\
18.70\end{array}$} & \multirow{3}{*}{$\begin{array}{c}\text { SE } \\
5.44\end{array}$} & \multicolumn{2}{|c|}{$95 \% \mathrm{CI}$} \\
\hline & & & & & $\mathbf{L} \mathbf{L}$ & UL & & & & $\mathbf{L L}$ & $\mathbf{U L}$ \\
\hline \multirow[b]{2}{*}{ Binge alcohol } & Binge use in the past month & & & & 11.73 & 43.90 & & & & 1.39 & 36.00 \\
\hline & $\begin{array}{l}\text { Never or 'no' binge use in } \\
\text { past month }\end{array}$ & 46 & 72.19 & 5.05 & 56.10 & 88.27 & 93 & 81.30 & 5.44 & 64.00 & 98.61 \\
\hline \multirow[b]{2}{*}{ Heavy alcohol } & Heavy use in the past month & 28 & 39.05 & 12.32 & 0.00 & 78.27 & 50 & 45.23 & 19.57 & 0.00 & 100.00 \\
\hline & $\begin{array}{l}\text { Never or 'no' heavy use in } \\
\text { past month }\end{array}$ & 43 & 60.95 & 12.32 & 21.73 & 100.00 & 79 & 54.77 & 19.57 & 0.00 & 100.00 \\
\hline \multirow{2}{*}{$\begin{array}{l}\text { Tranquilizers/ } \\
\text { sedatives }\end{array}$} & Used in the past month & 34 & 39.60 & 6.59 & 18.62 & 60.58 & 52 & 27.39 & 9.45 & 0.00 & 57.47 \\
\hline & Not used in the past month & 37 & 60.40 & 6.59 & 39.42 & 81.38 & 77 & 72.61 & 9.45 & 42.53 & 100.00 \\
\hline \multirow{2}{*}{ Cigarettes } & Used in the past month & 65 & 77.40 & 12.26 & 38.37 & 100.00 & 118 & 88.46 & 7.31 & 65.21 & 100.00 \\
\hline & Not used in the past month & 6 & 22.60 & 12.26 & 0.00 & 61.63 & 11 & 11.54 & 7.31 & 0.00 & 34.79 \\
\hline \multirow{2}{*}{$\begin{array}{l}\text { Other tobacco } \\
\text { products }\end{array}$} & Used in the past month & 28 & 37.05 & 3.98 & 24.40 & 49.70 & 52 & 41.29 & 2.73 & 32.60 & 49.97 \\
\hline & Not used in the past month & 43 & 62.95 & 3.98 & 50.30 & 75.60 & 77 & 58.71 & 2.73 & 50.03 & 67.40 \\
\hline \multirow{2}{*}{ Marijuana } & Used in the past month & 54 & 74.79 & 5.60 & 56.96 & 92.62 & 84 & 56.59 & 9.24 & 27.18 & 85.99 \\
\hline & Not used in the past month & 17 & 25.21 & 5.60 & 7.38 & 43.04 & 45 & 43.41 & 9.24 & 14.01 & 72.82 \\
\hline \multirow{2}{*}{ Cocaine } & Used in the past month & 35 & 47.26 & 10.45 & 14.00 & 80.52 & 58 & 51.80 & 5.31 & 34.91 & 68.68 \\
\hline & Not used in the past month & 36 & 52.74 & 10.45 & 19.48 & 86.00 & 71 & 48.20 & 5.31 & 31.32 & 65.09 \\
\hline \multirow{2}{*}{ Crack } & Used in the past month & 17 & 21.90 & 2.53 & 13.87 & 29.94 & 33 & 31.36 & 2.71 & 22.73 & 40.00 \\
\hline & Not used in the past month & 54 & 78.10 & 2.53 & 70.06 & 86.13 & 96 & 68.64 & 2.71 & 60.00 & 77.27 \\
\hline \multirow{2}{*}{$\begin{array}{c}\text { Hallucinogens/ } \\
\text { inhalants }\end{array}$} & Used in the past month & 21 & 27.21 & 1.56 & 22.25 & 32.17 & 31 & 13.72 & 1.00 & 10.55 & 16.88 \\
\hline & Not used in the past month & 50 & 72.79 & 1.56 & 67.83 & 77.75 & 98 & 86.28 & 1.00 & 83.12 & 89.45 \\
\hline \multirow{2}{*}{ Stimulants } & Used in the past month & 12 & 9.94 & 4.12 & 0.00 & 23.05 & 18 & 4.71 & 0.43 & 3.35 & 6.07 \\
\hline & Not used in the past month & 59 & 90.06 & 4.12 & 76.95 & 100.00 & 111 & 95.29 & 0.43 & 93.93 & 96.65 \\
\hline
\end{tabular}

Note: Full notes are at the end of ST 4.15. 
(ST 4.15 continued)

\begin{tabular}{|c|c|c|c|c|c|c|c|c|c|c|c|}
\hline \multirow{4}{*}{\multicolumn{2}{|c|}{ Core substances }} & \multicolumn{10}{|c|}{ Period } \\
\hline & & \multicolumn{5}{|c|}{ (3) 2010 to 2013} & \multicolumn{5}{|c|}{ (4) 2014 to 2017} \\
\hline & & \multirow{2}{*}{$\mathbf{n}$} & \multirow{2}{*}{$\%^{a}$} & \multirow{2}{*}{ SE } & \multicolumn{2}{|c|}{$95 \% \mathrm{CI}$} & \multirow{2}{*}{$\mathbf{n}$} & \multirow{2}{*}{$\%^{a}$} & \multirow{2}{*}{$\mathbf{S E}$} & \multicolumn{2}{|c|}{$95 \% \mathrm{CI}$} \\
\hline & & & & & $\mathbf{L} \mathbf{L}$ & $\mathbf{U L}$ & & & & $\mathbf{L L}$ & $\mathbf{U L}$ \\
\hline \multirow[b]{2}{*}{ Binge alcohol } & Binge use in the past month & 49 & 25.47 & 4.81 & 10.18 & 40.77 & 54 & 27.86 & 4.090 & 14.84 & 40.88 \\
\hline & $\begin{array}{l}\text { Never or 'no' binge use in } \\
\text { past month }\end{array}$ & 107 & 74.53 & 4.81 & 59.23 & 89.82 & 125 & 72.14 & 4.090 & 59.12 & 85.16 \\
\hline \multirow[b]{2}{*}{ Heavy alcohol } & Heavy use in the past month & 39 & 22.27 & 1.10 & 18.78 & 25.77 & 40 & 22.07 & 2.142 & 15.26 & 28.89 \\
\hline & $\begin{array}{l}\text { Never or 'no' heavy use in } \\
\text { past month }\end{array}$ & 117 & 77.73 & 1.10 & 74.23 & 81.22 & 139 & 77.93 & 2.142 & 71.11 & 84.74 \\
\hline \multirow{2}{*}{$\begin{array}{l}\text { Tranquilizers/ } \\
\text { sedatives }\end{array}$} & Used in the past month & 77 & 56.48 & 9.73 & 25.51 & 87.46 & 71 & 46.68 & 8.06 & 21.03 & 72.33 \\
\hline & Not used in the past month & 79 & 43.52 & 9.73 & 12.54 & 74.49 & 108 & 53.32 & 8.06 & 27.67 & 78.97 \\
\hline \multirow{2}{*}{ Cigarettes } & Used in the past month & 149 & 89.03 & 1.55 & 84.10 & 93.95 & 161 & 87.99 & 2.96 & 78.56 & 97.42 \\
\hline & Not used in the past month & 7 & 10.97 & 1.55 & 6.05 & 15.90 & 18 & 12.01 & 2.96 & 2.58 & 21.44 \\
\hline \multirow{2}{*}{$\begin{array}{l}\text { Other tobacco } \\
\text { products }\end{array}$} & Used in the past month & 59 & 41.02 & 2.07 & 34.45 & 47.60 & 65 & 35.98 & 1.32 & 31.79 & 40.18 \\
\hline & Not used in the past month & 97 & 58.98 & 2.07 & 52.40 & 65.55 & 114 & 64.02 & 1.32 & 59.82 & 68.21 \\
\hline \multirow{2}{*}{ Marijuana } & Used in the past month & 112 & 66.04 & 0.59 & 64.16 & 67.91 & 121 & 66.27 & 5.72 & 48.08 & 84.46 \\
\hline & Not used in the past month & 44 & 33.96 & 0.59 & 32.09 & 35.84 & 58 & 33.73 & 5.72 & 15.54 & 51.92 \\
\hline \multirow{2}{*}{ Cocaine } & Used in the past month & 69 & 45.96 & 0.23 & 45.22 & 46.70 & 68 & 41.73 & 6.90 & 19.77 & 63.68 \\
\hline & Not used in the past month & 87 & 54.04 & 0.23 & 53.30 & 54.78 & 111 & 58.27 & 6.90 & 36.32 & 80.23 \\
\hline \multirow{2}{*}{ Crack } & Used in the past month & 37 & 27.25 & 2.45 & 19.44 & 35.06 & 34 & 24.73 & 0.60 & 22.83 & 26.63 \\
\hline & Not used in the past month & 119 & 72.75 & 2.45 & 64.94 & 80.56 & 145 & 75.27 & 0.60 & 73.37 & 77.17 \\
\hline \multirow{2}{*}{$\begin{array}{l}\text { Hallucinogens/ } \\
\text { inhalants }\end{array}$} & Used in the past month & 30 & 25.17 & 10.77 & 0.00 & 59.45 & 21 & 7.64 & 0.83 & 5.00 & 10.28 \\
\hline & Not used in the past month & 126 & 74.83 & 10.77 & 40.55 & 100.00 & 158 & 92.36 & 0.83 & 89.72 & 95.00 \\
\hline \multirow{2}{*}{ Stimulants } & Used in the past month & 29 & 17.76 & 4.39 & 3.78 & 31.74 & 41 & 25.76 & 3.95 & 13.20 & 38.32 \\
\hline & Not used in the past month & 127 & 82.24 & 4.39 & 68.26 & 96.22 & 138 & 74.24 & 3.95 & 61.68 & 86.80 \\
\hline
\end{tabular}




\section{Table notes for ST 4.15}

Abbreviations: $\mathrm{n}=$ number of respondents, $\%=$ weighted prevalence; $\mathrm{SE}=$ standard error; $95 \% \mathrm{CI}=95 \%$ confidence interval; $\mathrm{LL}$ = lower limit; $\mathrm{UL}=$ upper limit

${ }^{a}$ All weighted prevalence are based on the total number of those who reported past month use of each core substance within each period 
ST 4.12a-c | Joinpoint regression results of longitudinal changes in prevalence of significantly different past month core substance use among past month combination opioid use group, NSDUH 2002 to $2017^{\mathrm{a}}$

a) Crack use $e^{\text {b }}$

\begin{tabular}{|c|c|c|c|c|c|c|}
\hline Segment & $\begin{array}{c}\text { Lower } \\
\text { endpoint }\end{array}$ & $\begin{array}{c}\text { Upper } \\
\text { endpoint }\end{array}$ & Slope & SE & $\begin{array}{c}\text { Test } \\
\text { statistic (t) }\end{array}$ & p-value \\
\hline $\mathbf{1}$ & 2002 & 2017 & 0.46 & 0.1 & 3.5 & 0.004 \\
\hline
\end{tabular}

b) Hallucinogen and/or inhalant use

\begin{tabular}{|c|c|c|c|c|c|c|}
\hline Segment & $\begin{array}{c}\text { Lower } \\
\text { endpoint }\end{array}$ & $\begin{array}{c}\text { Upper } \\
\text { endpoint }\end{array}$ & Slope & SE & $\begin{array}{c}\text { Test } \\
\text { statistic (t) }\end{array}$ & p-value \\
\hline $\mathbf{1}$ & 2002 & 2006 & -3.733 & 3.58 & -1.04 & 0.33 \\
\hline $\mathbf{2}$ & 2006 & 2011 & 3.0843 & 4.90 & 0.63 & 0.55 \\
\hline $\mathbf{3}$ & 2011 & 2017 & $-3.985^{\wedge}$ & 1.25 & -3.18 & 0.013 \\
\hline
\end{tabular}

c) Stimulant use $e^{\mathrm{b}}$

\begin{tabular}{|c|c|c|c|c|c|c|}
\hline Segment & $\begin{array}{c}\text { Lower } \\
\text { endpoint }\end{array}$ & $\begin{array}{c}\text { Upper } \\
\text { endpoint }\end{array}$ & Slope & SE & $\begin{array}{c}\text { Test } \\
\text { statistic (t) }\end{array}$ & p-value \\
\hline $\mathbf{1}$ & 2002 & 2009 & $0.034^{\wedge}$ & 0.0092 & 3.69 & 0.0036 \\
\hline $\mathbf{2}$ & 2009 & 2017 & $0.253^{\wedge}$ & 0.0458 & 5.53 & 0.00018 \\
\hline
\end{tabular}

Abbreviations: $\mathrm{APC}=$ annual percentage change; $\mathrm{SE}=$ standard error; $\mathrm{CI}=$ confidence interval ${ }^{a}$ Two continuous variables were used to perform the joinpoint regression: the weighted prevalence of past month core substance use and survey year for among PWUO. ${ }^{\wedge}$ Slope is significantly different from zero at alpha $=0.05$. 
ST 4.13 Comparison of different alcohol use levels by longitudinal prevalence differences and predicting PWUO/opioid use group class membership (for Aim 1) (n=16,985), NSDUH 2002 to 2017

\begin{tabular}{|c|c|c|c|c|c|c|c|c|}
\hline \multirow{5}{*}{$\begin{array}{c}\text { Alcohol } \\
\text { Level }\end{array}$} & \multicolumn{8}{|c|}{ Significance } \\
\hline & \multicolumn{8}{|c|}{ Aim 1} \\
\hline & \multirow{2}{*}{\multicolumn{2}{|c|}{ PWUO }} & \multicolumn{6}{|c|}{ Opioid use groups } \\
\hline & & & \multicolumn{2}{|c|}{ PPR-only } & \multicolumn{2}{|c|}{ Heroin-only } & \multicolumn{2}{|c|}{ Combination opioid } \\
\hline & $\begin{array}{l}\chi^{2}(\mathrm{df}), \mathrm{p}- \\
\text { value }\end{array}$ & LR (p-value) & $\begin{array}{l}\chi^{2}(\mathrm{df}), \mathrm{p}- \\
\text { value }\end{array}$ & LR (p-value) & $\begin{array}{l}\chi^{2}(\mathrm{df}) \\
\mathrm{p} \text {-value }\end{array}$ & LR (p-value) & $\begin{array}{l}\chi^{2}(\mathrm{df}), \\
\mathrm{p} \text {-value }\end{array}$ & LR (p-value) \\
\hline Heavy & $\begin{array}{l}38.31(3), \\
<0.0001\end{array}$ & $\begin{array}{c}<\mathbf{0 . 0 0 0 1} \\
\text { (compared to } \\
\text { non-PWUO) }\end{array}$ & $\begin{array}{l}27.26(3), \\
<0.0001\end{array}$ & $\begin{array}{c}\mathbf{0 . 0 1 1} \\
\text { (compared to } \\
\text { heroin-only); } \\
0.28 \text { (compared } \\
\text { to combination) }\end{array}$ & $\begin{array}{c}8.13(3), \\
0.043\end{array}$ & $\begin{array}{c}\mathbf{0 . 0 1 1} \\
\text { (compared to } \\
\text { PPR-only); } \\
0.082 \\
\text { (compared to } \\
\text { heroin-only) }\end{array}$ & $\begin{array}{c}3.07(3) \\
0.38\end{array}$ & $\begin{array}{c}0.28 \text { (compared } \\
\text { to PPR-only); } \\
0.082 \text { (compared } \\
\text { to heroin-only) }\end{array}$ \\
\hline Binge & $\begin{array}{c}0.93(3) \\
0.82\end{array}$ & $\begin{array}{c}<\mathbf{0 . 0 0 0 1} \\
\text { (compared to } \\
\text { non-PWUO) }\end{array}$ & $\begin{array}{c}1.40(3) \\
0.70\end{array}$ & $\begin{array}{c}<\mathbf{0 . 0 0 0 1} \\
\text { (compared to } \\
\text { heroin-only); } \\
0.076 \\
\text { (compared to } \\
\text { combination) }\end{array}$ & $\begin{array}{c}3.88(3) \\
0.27\end{array}$ & $\begin{array}{c}<\mathbf{0 . 0 0 0 1} \\
\text { (compared to } \\
\text { PPR-only); } \\
\text { 0.37 (compared } \\
\text { to combination) }\end{array}$ & $\begin{array}{c}2.56(3) \\
0.46\end{array}$ & $\begin{array}{c}0.0758 \\
\text { (compared to } \\
\text { PPR-only); } 0.37 \\
\text { (compared to } \\
\text { heroin-only) }\end{array}$ \\
\hline $\begin{array}{c}\text { Past } \\
\text { month }\end{array}$ & $\begin{array}{c}2.41(3) \\
0.49\end{array}$ & $\begin{array}{c}<\mathbf{0 . 0 0 0 1} \\
\text { (compared to } \\
\text { non-PWUO) }\end{array}$ & $\begin{array}{c}2.63(3) \\
0.46\end{array}$ & $\begin{array}{c}\mathbf{0 . 0 0 6 2} \\
\text { (compared to } \\
\text { heroin-only); } \\
0.82 \text { (compared } \\
\text { to combination) }\end{array}$ & $\begin{array}{c}3.30(3) \\
0.35\end{array}$ & $\begin{array}{c}\mathbf{0 . 0 0 6 2} \\
\text { (compared to } \\
\text { PPR-only); } \\
\text { 0.63 (compared } \\
\text { to combination) }\end{array}$ & $\begin{array}{c}1.91(3) \\
0.59\end{array}$ & $\begin{array}{l}0.82 \text { (compared } \\
\text { to PPR-only); } \\
0.63 \text { (compared } \\
\text { to heroin-only) }\end{array}$ \\
\hline
\end{tabular}

Abbreviations: $\chi^{2}(\mathrm{df})=$ Chi-square (degrees of freedom); LR $=$ logistic regression

Note: Bolded values denote statistical significance at $\mathrm{p}<0.05$. 
ST 4.14 | Fit statistics of latent class models with up to 8 classes using 11 categorical core substances from the NSDUH $(\mathrm{n}=$ 16,985), 2002 to 2017

\begin{tabular}{|c|c|c|c|c|c|c|c|c|c|}
\hline \multirow{2}{*}{ Classes } & \multirow{2}{*}{$\mathbf{n}$} & \multirow{2}{*}{ Parameters } & \multirow{2}{*}{$\begin{array}{c}\text { Log } \\
\text { Likelihood }\end{array}$} & \multicolumn{2}{|c|}{$\begin{array}{c}\text { Lo, Mendell, Rubin } \\
\text { Likelihood Ratio } \\
\text { Test }\end{array}$} & \multirow{2}{*}{ AIC } & \multirow{2}{*}{ BIC } & \multirow{2}{*}{ ssBIC } & \multirow{2}{*}{ Entropy } \\
\hline & & & & $\begin{array}{l}\text { Classes } \\
\text { compared }\end{array}$ & p-value & & & & \\
\hline 1 & 16985 & 12 & -38103.378 & $\mathrm{n} / \mathrm{a}$ & $\mathrm{n} / \mathrm{a}$ & 76230.756 & 76313.893 & 76275.760 & $\mathrm{n} / \mathrm{a}$ \\
\hline 2 & 16985 & 25 & -35816.954 & 1 versus 2 & 0.2063 & 71683.907 & 71857.11 & 71777.665 & 0.653 \\
\hline 3 & 16985 & 38 & -34868.002 & 2 versus 3 & 0.223 & 69812.004 & 70075.272 & 69954.516 & 0.752 \\
\hline 4 & 16985 & 51 & -34139.581 & 3 versus 4 & 0.3876 & 68381.162 & 68734.496 & 68572.429 & 0.804 \\
\hline 5 & 16985 & 64 & -33729.321 & 4 versus 5 & 0.5195 & 67586.641 & 68030.04 & 67826.662 & 0.829 \\
\hline 6 & 16985 & 77 & -33534.328 & 5 versus 6 & 0.5283 & 67222.656 & 67756.121 & 67511.431 & 0.779 \\
\hline 7 & 16985 & 90 & -33457.214 & 6 versus 7 & 0.606 & 67094.427 & 67717.957 & 67431.956 & 0.794 \\
\hline 8 & 16985 & 103 & -33390.517 & 7 versus 8 & 0.5693 & 66987.034 & 67700.629 & 67373.317 & 0.757 \\
\hline
\end{tabular}

Abbreviations: AIC = Akaike Information Criterion; BIC = Bayesian Information Criterion; ssBIC = sample size-adjusted Bayesian Information Criterion 
ST 4.15 | Joinpoint regression results of longitudinal changes in prevalence of Class 1, NSDUH 2002 to $2017^{\mathrm{a}}$

\begin{tabular}{|c|c|c|c|c|c|c|}
\hline Segment & $\begin{array}{c}\text { Lower } \\
\text { endpoint }\end{array}$ & $\begin{array}{c}\text { Upper } \\
\text { endpoint }\end{array}$ & Slope & SE & $\begin{array}{c}\text { Test } \\
\text { statistic } \\
(\mathbf{t})\end{array}$ & p-value \\
\hline $\mathbf{1}$ & 2002 & 2009 & 0.04 & 0.5 & 0.1 & 0.939 \\
\hline $\mathbf{2}$ & 2009 & 2012 & 1.63 & 0.8 & 2.1 & 0.07 \\
\hline $\mathbf{3}$ & 2012 & 2017 & $-0.71^{\wedge}$ & 0.17 & -4.16 & $<0.001$ \\
\hline
\end{tabular}

Abbreviations: $\mathrm{SE}=$ standard error; $\mathrm{CI}=$ confidence interval

${ }^{a}$ Two continuous variables were used to perform the joinpoint regression: the weighted prevalence of Class 1 and survey year.

${ }^{\wedge}$ Slope is significantly different from zero at alpha $=0.05$.

ST 4.16 Joinpoint regression results of longitudinal changes in prevalence of Class 2, NSDUH 2002 to $2017^{\mathrm{a}}$

\begin{tabular}{|c|c|c|c|c|c|c|}
\hline Segment & $\begin{array}{c}\text { Lower } \\
\text { endpoint }\end{array}$ & $\begin{array}{c}\text { Upper } \\
\text { endpoint }\end{array}$ & Slope & SE & $\begin{array}{c}\text { Test } \\
\text { statistic } \\
(\mathbf{t})\end{array}$ & p-value \\
\hline $\mathbf{1}$ & 2002 & 2009 & $0.87^{\wedge}$ & 0.3 & 2.5 & 0.029 \\
\hline $\mathbf{2}$ & 2009 & 2017 & $-0.3^{\wedge}$ & 0.0 & -32.8 & $<0.001$ \\
\hline
\end{tabular}

Abbreviations: $\mathrm{SE}=$ standard error; $\mathrm{CI}=$ confidence interval

${ }^{a}$ Two continuous variables were used to perform the joinpoint regression: the weighted prevalence of Class 2 and survey year.

${ }^{\wedge}$ Slope is significantly different from zero at alpha $=0.05$.

ST 4.17 | Joinpoint regression results of longitudinal changes in prevalence of Class 3, NSDUH 2002 to $2017^{\mathrm{a}}$

\begin{tabular}{|c|c|c|c|c|c|c|}
\hline Segment & $\begin{array}{c}\text { Lower } \\
\text { endpoint }\end{array}$ & $\begin{array}{c}\text { Upper } \\
\text { endpoint }\end{array}$ & Slope & SE & $\begin{array}{c}\text { Test } \\
\text { statistic } \\
(\mathbf{t})\end{array}$ & p-value \\
\hline $\mathbf{1}$ & 2002 & 2010 & 0.05 & 0.2 & 0.2 & 0.840 \\
\hline $\mathbf{2}$ & 2010 & 2017 & $1.06^{\wedge}$ & 0.2 & 4.9 & $<0.001$ \\
\hline
\end{tabular}

Abbreviations: $\mathrm{SE}=$ standard error; $\mathrm{CI}=$ confidence interval ${ }^{a}$ Two continuous variables were used to perform the joinpoint regression: the weighted prevalence of Class 3 and survey year.

${ }^{\wedge}$ Slope is significantly different from zero at alpha $=0.05$. 
ST 4.18 | Joinpoint regression results of longitudinal changes in prevalence of Class 4, NSDUH 2002 to $2017^{\mathrm{a}}$

\begin{tabular}{|c|c|c|c|c|c|c|}
\hline Segment & $\begin{array}{c}\text { Lower } \\
\text { endpoint }\end{array}$ & $\begin{array}{c}\text { Upper } \\
\text { endpoint }\end{array}$ & Slope & SE & $\begin{array}{c}\text { Test } \\
\text { statistic } \\
(\mathbf{t})\end{array}$ & p-value \\
\hline $\mathbf{1}$ & 2002 & 2017 & $-0.32^{\wedge}$ & 0.1 & -4.1 & 0.001 \\
\hline
\end{tabular}

Abbreviations: $\mathrm{SE}=$ standard error; $\mathrm{CI}=$ confidence interval

${ }^{a}$ Two continuous variables were used to perform the joinpoint regression: the weighted prevalence of Class 4 and survey year.

${ }^{\wedge}$ Slope is significantly different from zero at alpha $=0.05$. 
ST 4.19| Fit latent class models with up to 8 classes to the 11 categorical core substances $(n=7,541)$, NSDUH 2010 to 2017

\begin{tabular}{|c|c|c|c|c|c|c|c|c|c|}
\hline \multirow[t]{2}{*}{ Classes } & \multirow[t]{2}{*}{$\mathbf{N}$} & \multirow[t]{2}{*}{ Parameters } & \multirow{2}{*}{$\begin{array}{c}\text { Log } \\
\text { Likelihood }\end{array}$} & \multicolumn{2}{|c|}{$\begin{array}{c}\text { Lo, Mendell, Rubin } \\
\text { Likelihood Ratio } \\
\text { Test }\end{array}$} & \multirow[t]{2}{*}{ AIC } & \multirow[t]{2}{*}{ BIC } & \multirow[t]{2}{*}{ ssBIC } & \multirow{2}{*}{ Entropy } \\
\hline & & & & $\begin{array}{l}\text { Classes } \\
\text { compared }\end{array}$ & p-value & & & & \\
\hline 1 & 16985 & 11 & -33602.112 & & & 67226.225 & 67302.434 & 67267.478 & \\
\hline 2 & 16985 & 23 & -31315.958 & 1 versus 2 & 0.1432 & 62677.917 & 62837.263 & 62764.174 & 0.654 \\
\hline 3 & 16985 & 35 & -30374.076 & 2 versus 3 & 0.1565 & 60818.152 & 61060.636 & 60949.413 & 0.753 \\
\hline 4 & 16985 & 47 & -29971.592 & 3 versus 4 & 0.4046 & 60037.184 & 60362.805 & 60213.449 & 0.794 \\
\hline 5 & 16985 & 59 & -29807.924 & 4 versus 5 & 0.619 & 59733.849 & 60142.607 & 59955.118 & 0.737 \\
\hline 6 & 16985 & 71 & -29737.842 & 5 versus 6 & 0.5635 & 59617.683 & 60109.579 & 59883.956 & 0.76 \\
\hline 7 & 16985 & 83 & -29671.762 & 6 versus 7 & 0.5964 & 59509.524 & 60084.557 & 59820.801 & 0.72 \\
\hline 8 & 16985 & 95 & -29629.711 & 7 versus 8 & 0.7003 & 59449.423 & 60107.593 & 59805.703 & 0.711 \\
\hline
\end{tabular}

Abbreviations: AIC = Akaike Information Criterion; BIC = Bayesian Information Criterion; ssBIC = sample size-adjusted Bayesian Information Criterion 
Table 4.20 | Overall size and weighted prevalence of the latent polydrug use subpopulations among PWUO ( $\mathrm{n}=7,541)$, NSDUH 2010 to 2017

\begin{tabular}{|c|c|c|c|c|c|}
\hline \multirow{2}{*}{ Polydrug latent class } & \multirow{2}{*}{$\mathbf{n}$} & \multirow{2}{*}{$\%$} & \multirow{2}{*}{ SE } & \multicolumn{2}{|c|}{$95 \% \mathrm{CI}$} \\
\hline & & & & LL & UL \\
\hline 1: mainly PPR & 3432 & 54.88 & 0.42 & 53.07 & 56.69 \\
\hline $\begin{array}{l}\text { 2: PPR - heavy alcohol - benzo- } \\
\text { stimulant - marijuana }\end{array}$ & 3508 & 37.02 & 0.20 & 36.14 & 37.89 \\
\hline 3: Heroin - marijuana & 341 & 4.22 & 0.27 & 3.03 & 5.40 \\
\hline 4: Combination opioid - polydrug & 260 & 3.88 & 0.32 & 2.52 & 5.25 \\
\hline
\end{tabular}

Note: $\mathrm{n}=$ total number of survey respondents; $\%=$ weighted percentage; $\mathrm{SE}=$ standard error; 95\% CI $=95 \%$ confidence interval $; \mathrm{LL}=$ lower limit; $\mathrm{UL}=$ upper limit

Table 4.21 | Endorsement probabilities for each core substance by latent polydrug class $(\mathrm{n}=7,541)$, NSDUH 2010 to 2017

\begin{tabular}{|c|c|c|c|c|}
\hline \multirow{2}{*}{$\begin{array}{c}\text { Substances used in the } \\
\text { past 30-days }\end{array}$} & 1: mainly PPR & $\begin{array}{c}\text { Polydrug latent classes among PWUO } \\
\text { 2: PPR - heavy } \\
\text { alcohol - benzo- } \\
\text { stimulant - } \\
\text { marijuana }\end{array}$ & $\begin{array}{c}\text { 3: Heroin - } \\
\text { marijuana }\end{array}$ & $\begin{array}{c}\text { Combination } \\
\text { opioid - } \\
\text { polydrug }\end{array}$ \\
\hline PPR & 1 & 1 & 0.121 & 0.741 \\
\hline Heroin & 0 & 0.058 & 1 & 0.605 \\
\hline Heavy alcohol & 0.112 & 0.391 & 0.09 & 0.307 \\
\hline Cigarettes & 0.339 & 0.83 & 0.87 & 0.879 \\
\hline Other tobacco products & 0.101 & 0.395 & 0.229 & 0.325 \\
\hline Tranquilizers/sedatives & 0.101 & 0.341 & 0.147 & 0.438 \\
\hline Stimulants & 0.022 & 0.155 & 0.05 & 0.146 \\
\hline Marijuana & 0.149 & 0.76 & 0.482 & 0.667 \\
\hline Cocaine & 0.007 & 0.133 & 0.147 & 1 \\
\hline Crack & 0 & 0 & 0 & 0.858 \\
\hline Hallucinogens/inhalants & 0.012 & 0.136 & 0.069 & 0.185 \\
\hline
\end{tabular}


APPENDIX B: Supplemental Graphs (SG) 
SG 4.1a-d | Differences in prevalence and standard errors of reported substance use for period 1 (2002-2005) among all opioid use groups for a) alcohol drinking, b) cigarette, other tobacco, and marijuana use, c) prescription stimulant and tranquilizer/sedative use, and d) illicit substance (cocaine, crack, hallucinogens/inhalants)

a) alcohol drinking

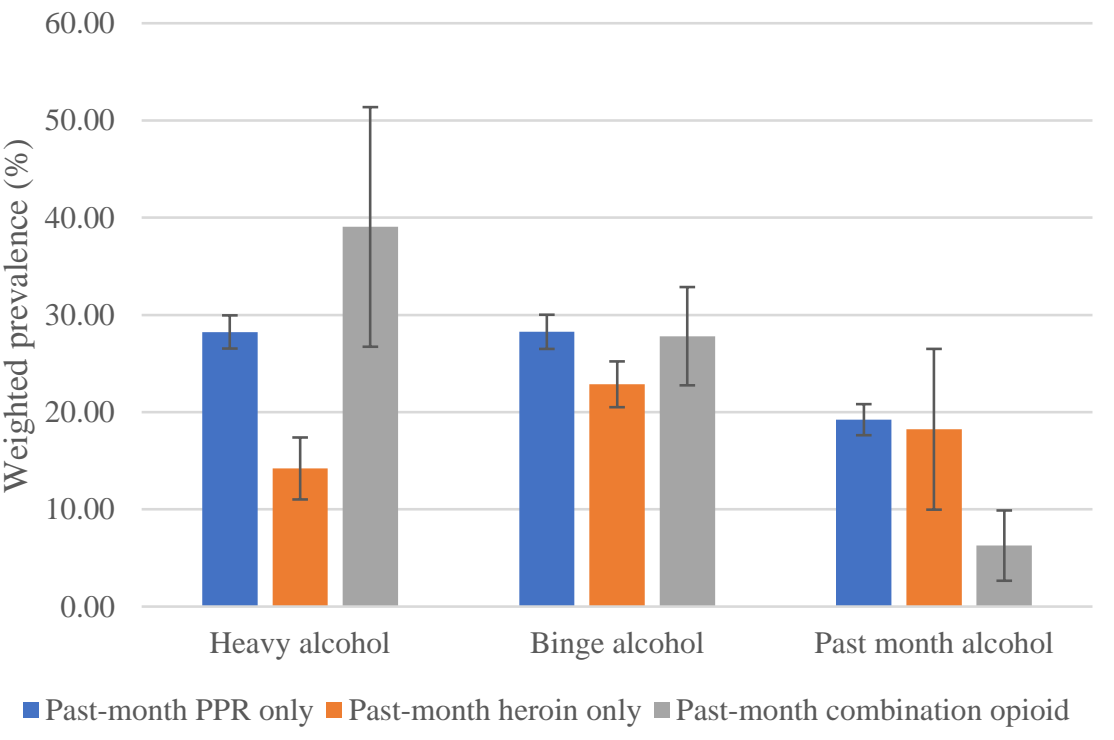

b) cigarette, other tobacco, and marijuana use

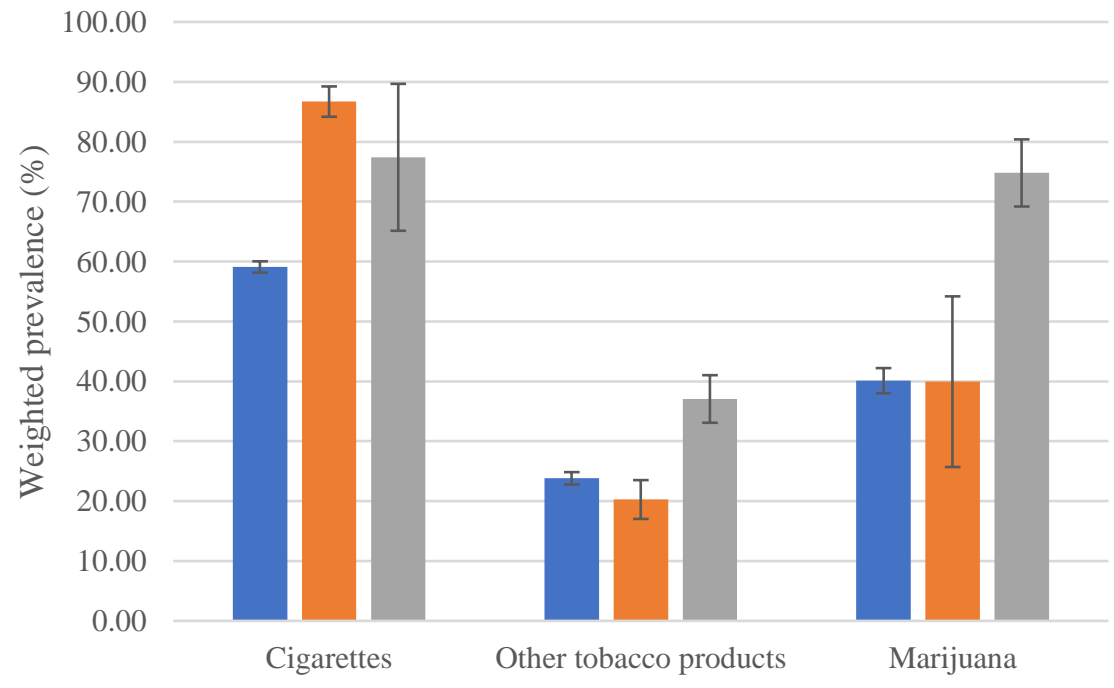

a Past-month PPR only a Past-month heroin only a Past-month combination opioid 
c) prescription stimulant and tranquilizer/sedative use

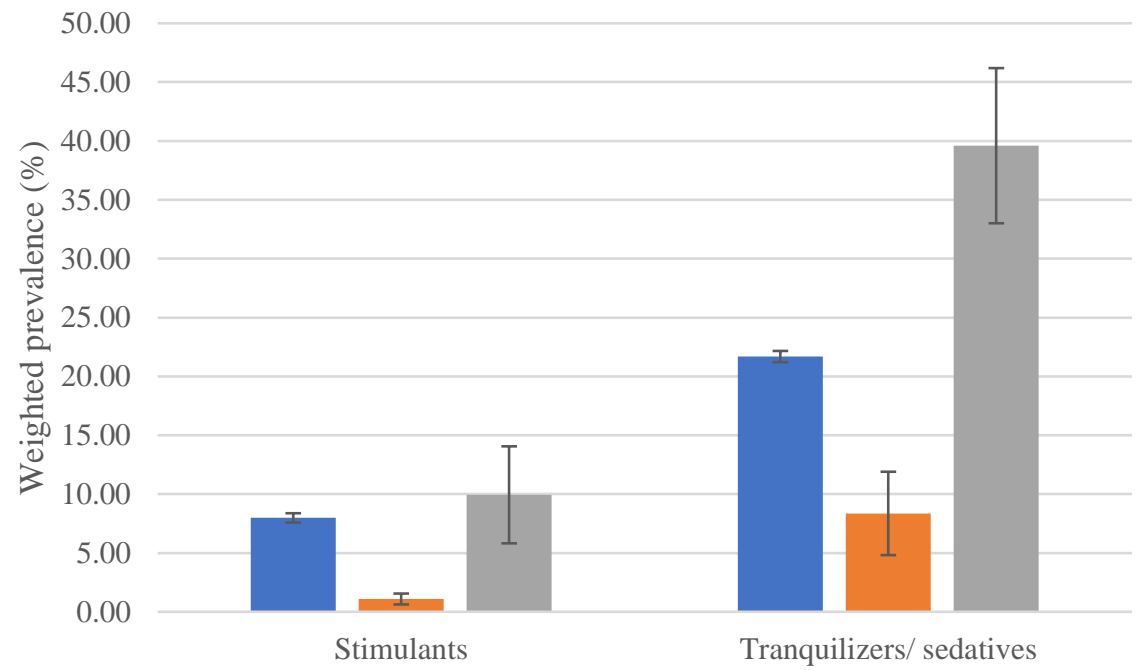

घ Past-month PPR only $\square$ Past-month heroin only $\square$ Past-month combination opioid

d) illicit substance use

70.00

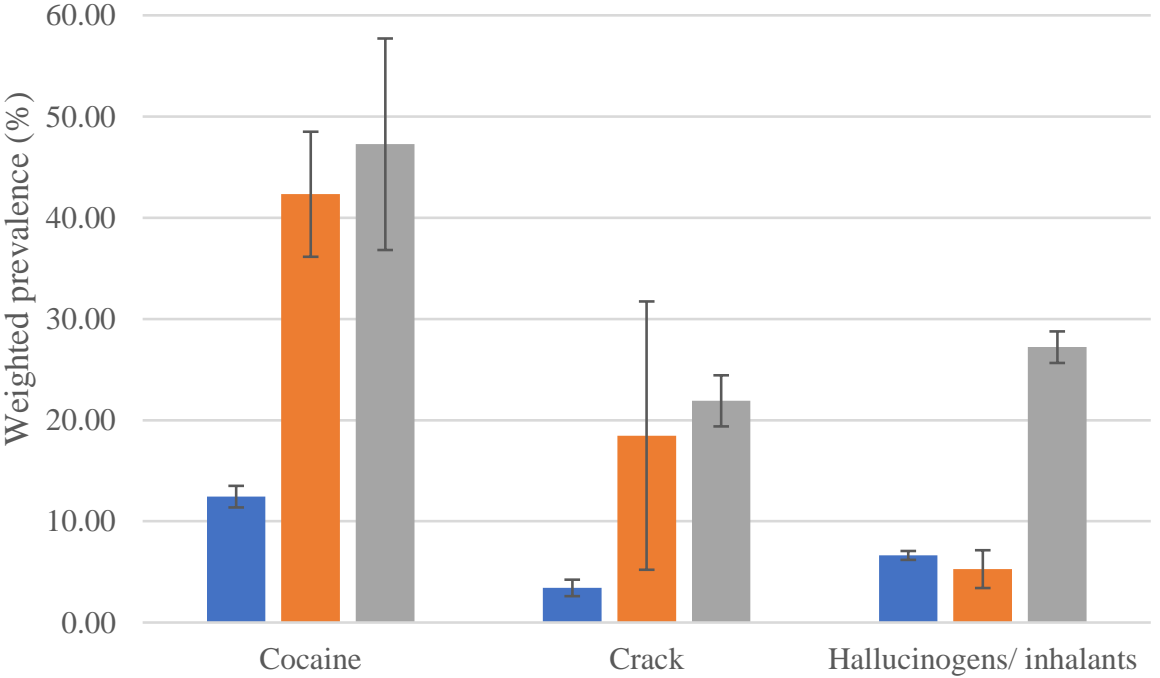

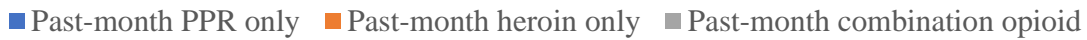


SG 4.2a-d | Differences in prevalence and standard errors of reported substance use for period 2 (2006-2009) among all opioid use groups for a) alcohol drinking, b) cigarette, other tobacco, and marijuana use, c) prescription stimulant and tranquilizer/sedative use, and d) illicit substance (cocaine, crack, hallucinogens/inhalants)

\section{a) alcohol drinking}

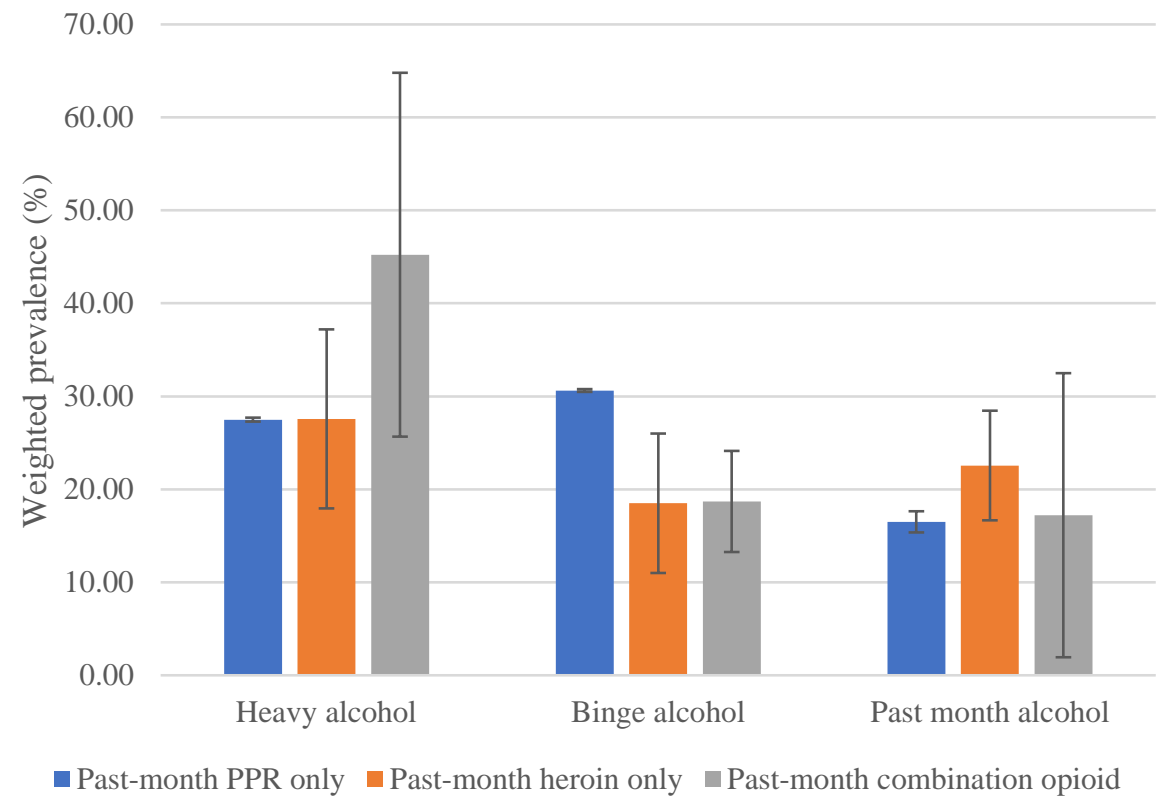

\section{b) cigarette, other tobacco, and marijuana use}

120.00

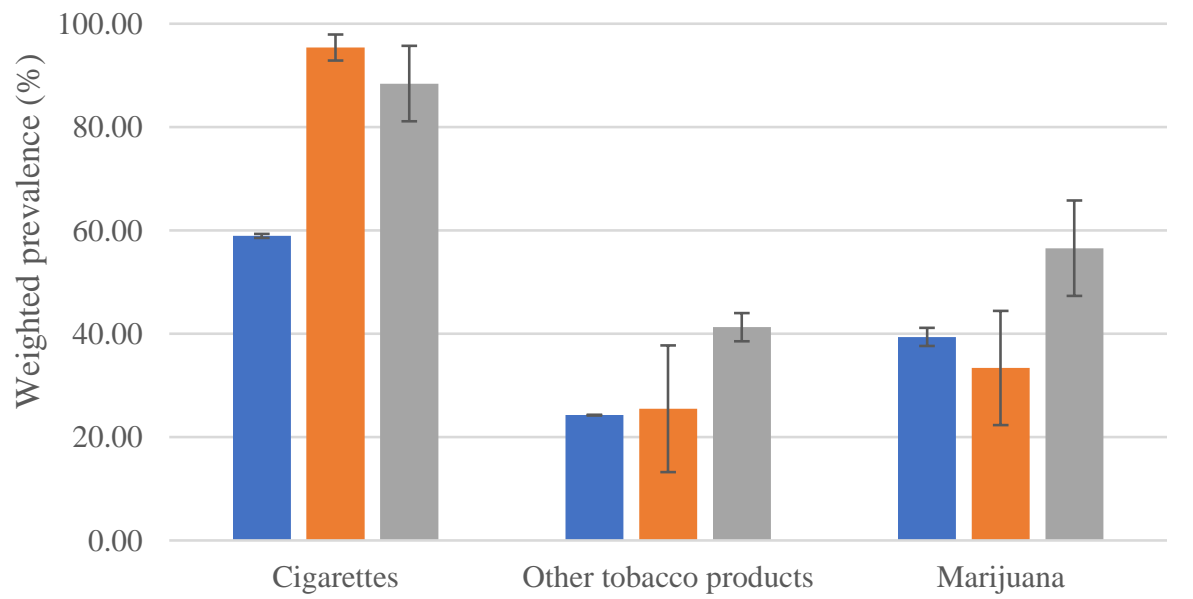

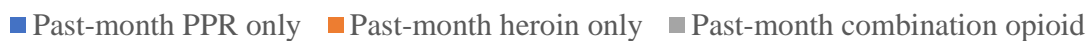




\section{c) prescription stimulant and tranquilizer/sedative use}

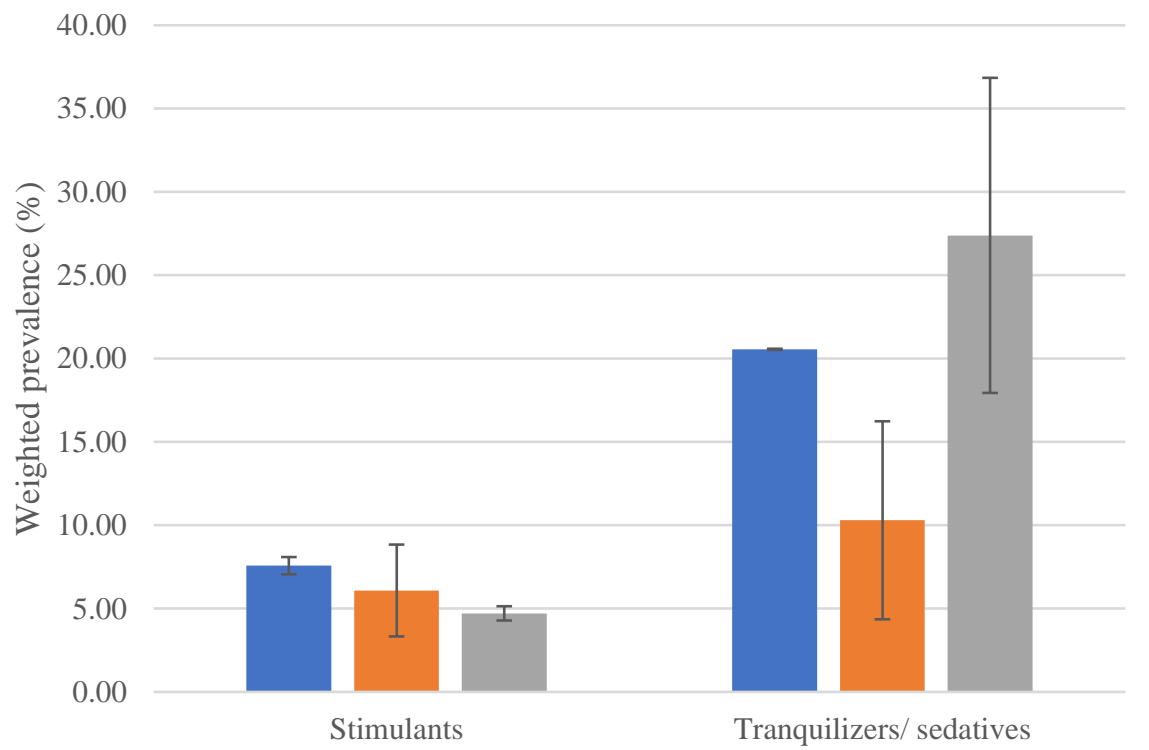

- Past-month PPR only a Past-month heroin only $\square$ Past-month combination opioid

\section{d) illicit substance use}

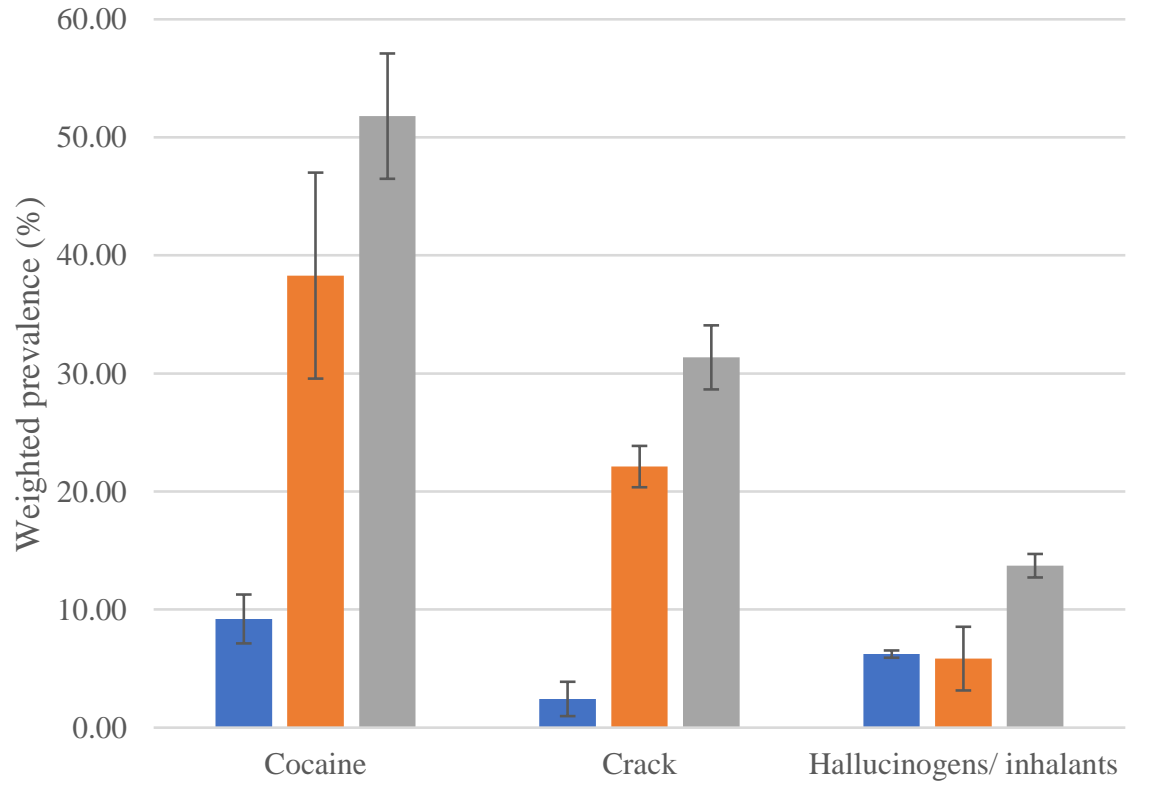

- Past-month PPR only $\square$ Past-month heroin only $\square$ Past-month combination opioid 
SG 4.3a-d | Differences in prevalence and standard errors of reported substance use for period 3 (2010-2013) among all opioid use groups for a) alcohol drinking, b) cigarette, other tobacco, and marijuana use, c) prescription stimulant and tranquilizer/sedative use, and d) illicit substance (cocaine, crack, hallucinogens/inhalants)

\section{a) alcohol drinking}

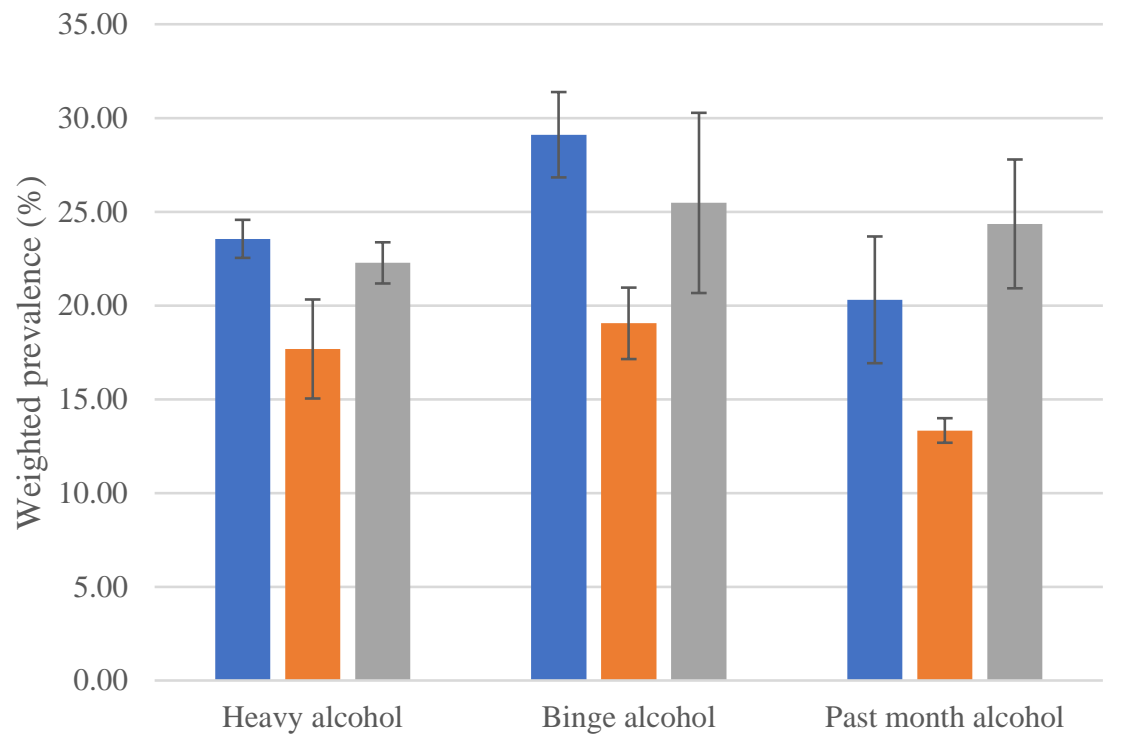

- Past-month PPR only $\square$ Past-month heroin only $\square$ Past-month combination opioid

\section{b) cigarette, other tobacco, and marijuana use}

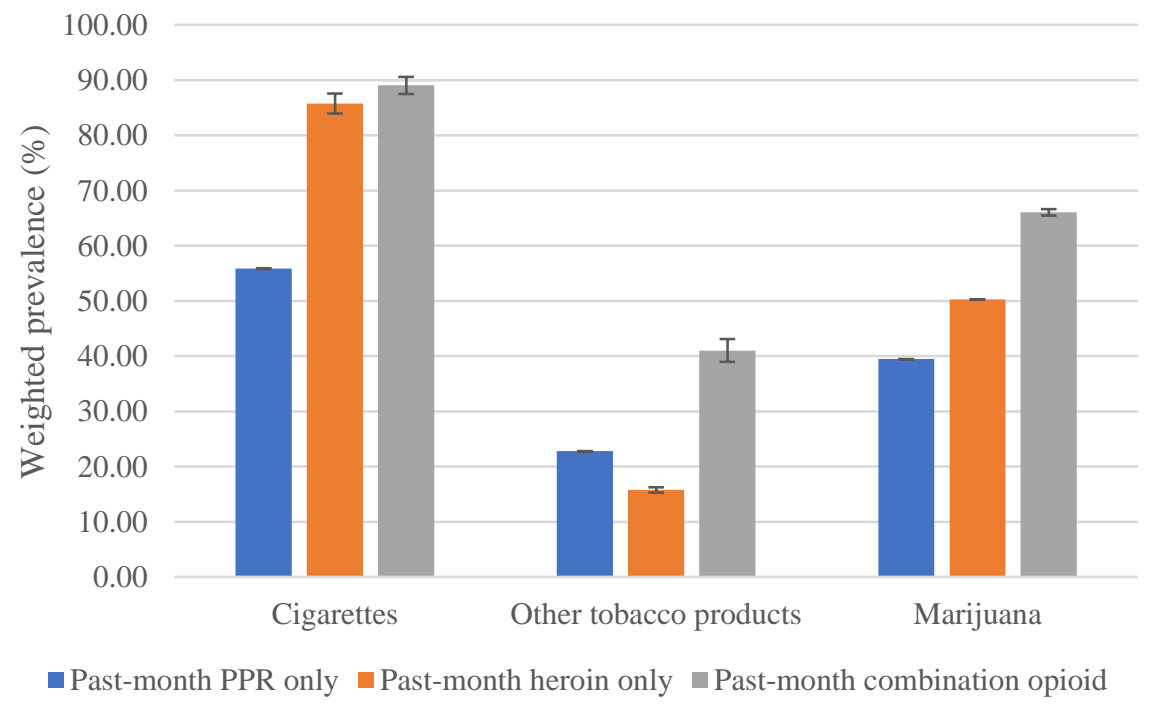


c) prescription stimulant and tranquilizer/sedative use

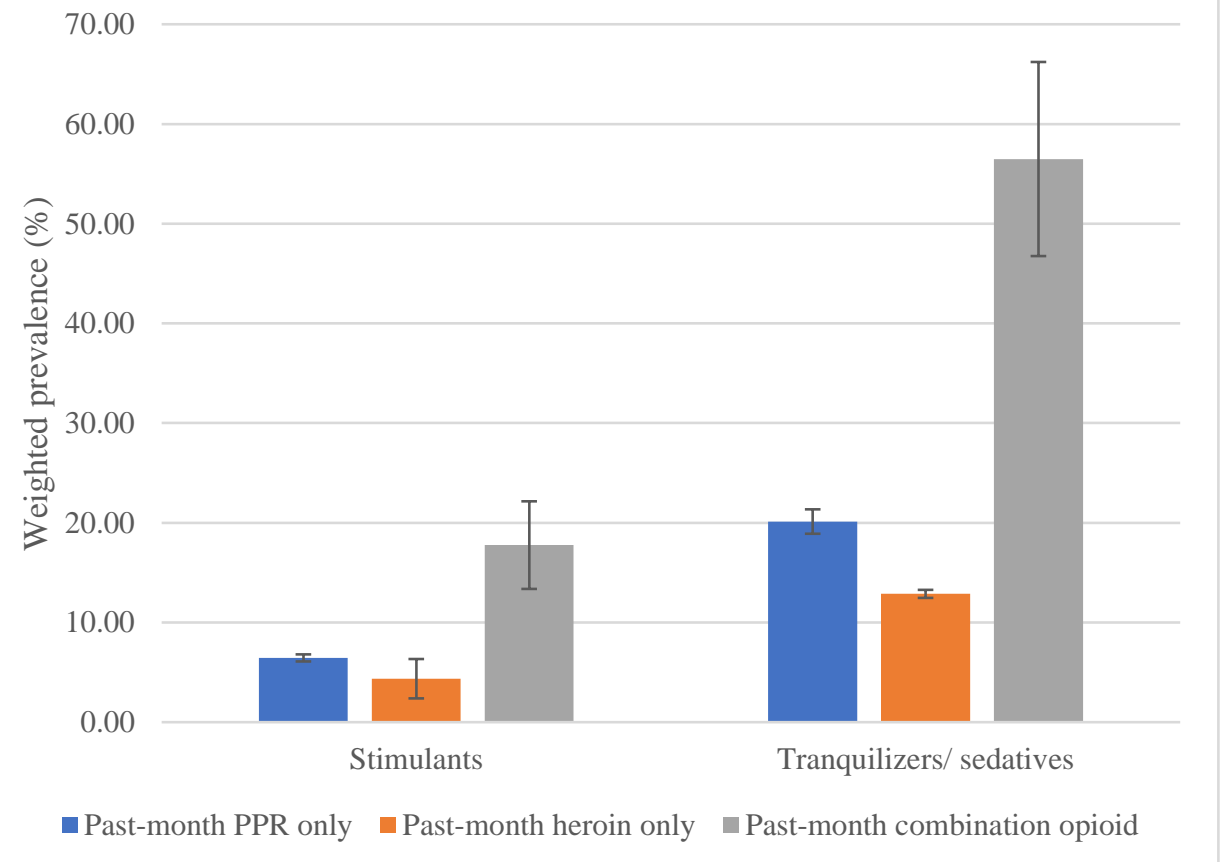

\section{d) illicit substance use}

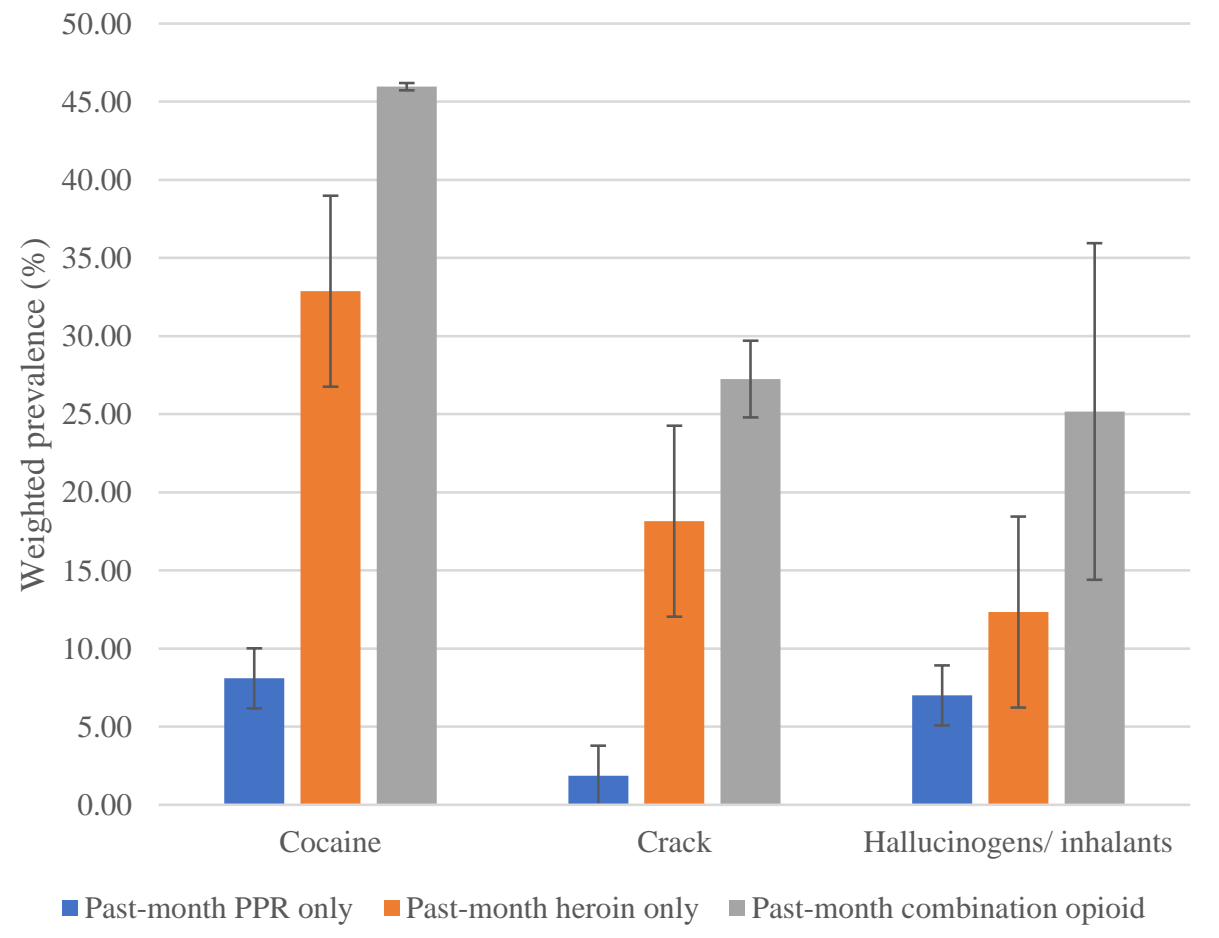


SG 4.4a-d | Differences in prevalence and standard errors of reported substance use for period 4 (2014-2017) among all opioid use groups for a) alcohol drinking, b) cigarette, other tobacco, and marijuana use, c) prescription stimulant and tranquilizer/sedative use, and d) illicit substance (cocaine, crack, hallucinogens/inhalants)

\section{a) alcohol drinking}

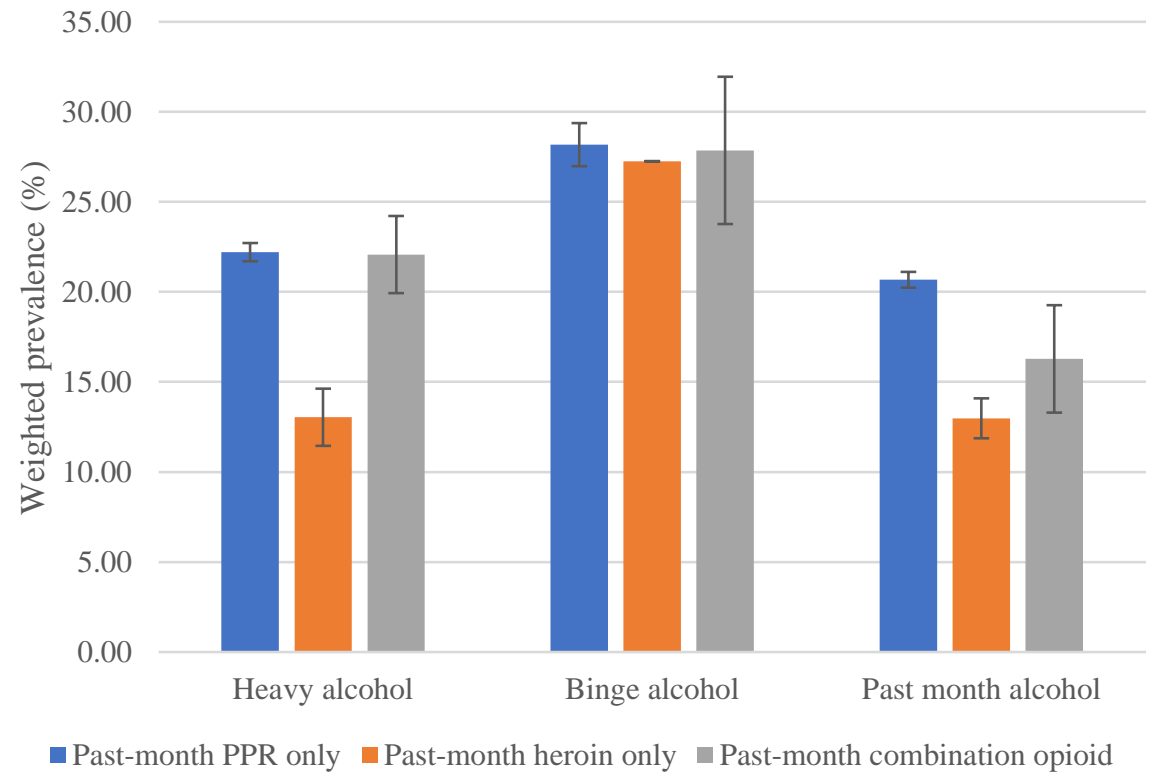

\section{b) cigarette, other tobacco, and marijuana use}

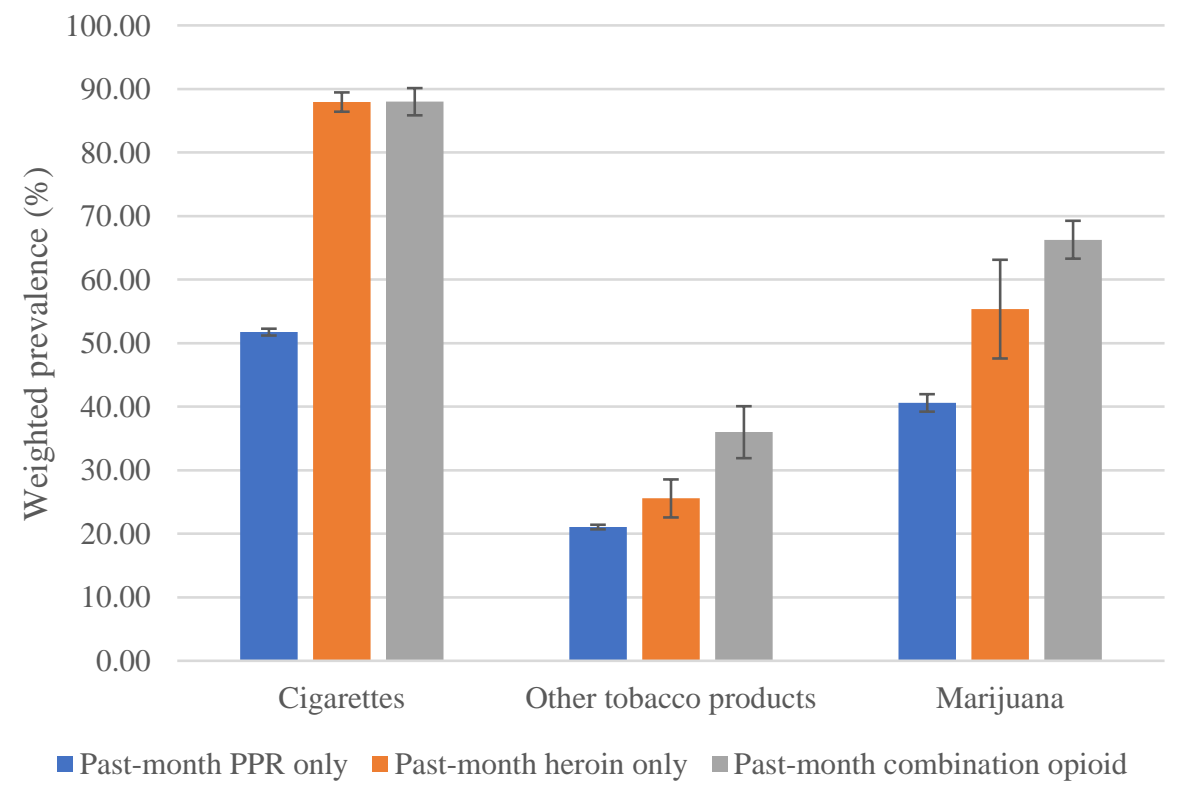




\section{c) prescription stimulant and tranquilizer/sedative use}

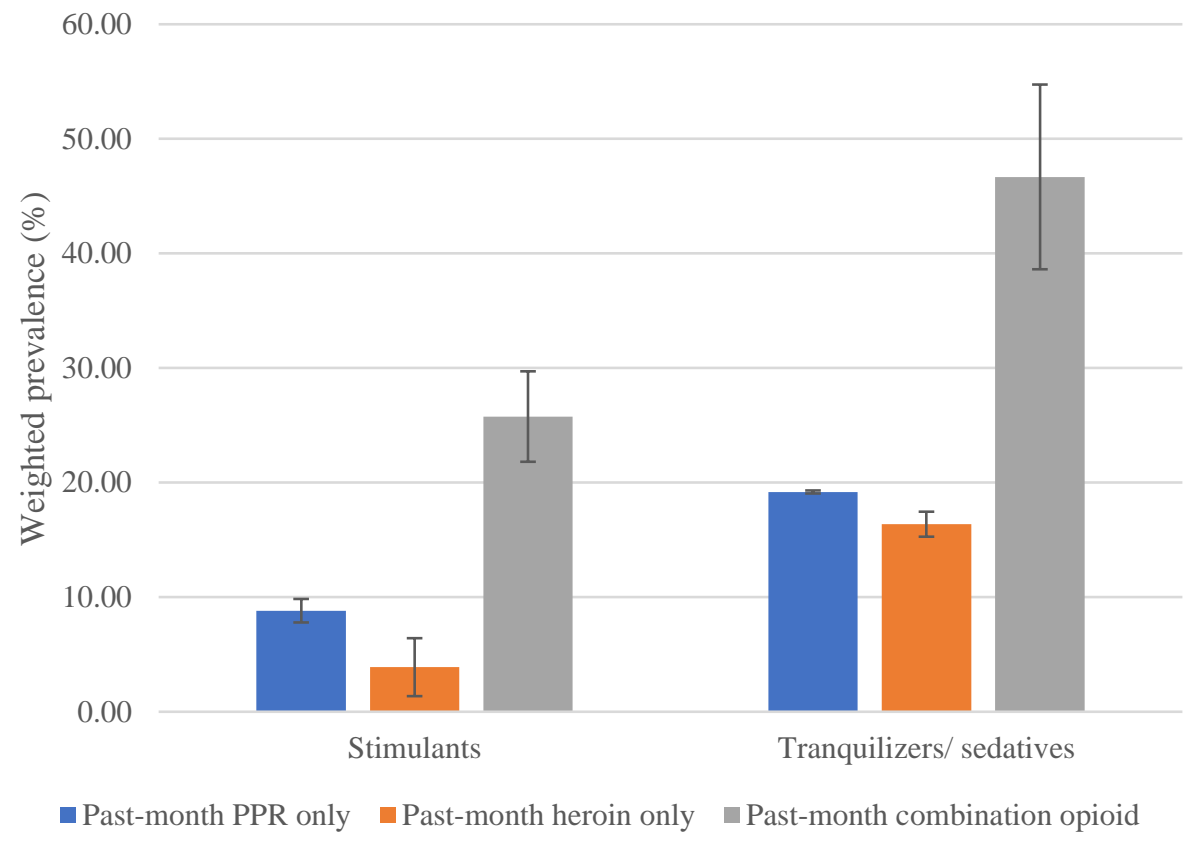

\section{d) illicit substance use}

60.00

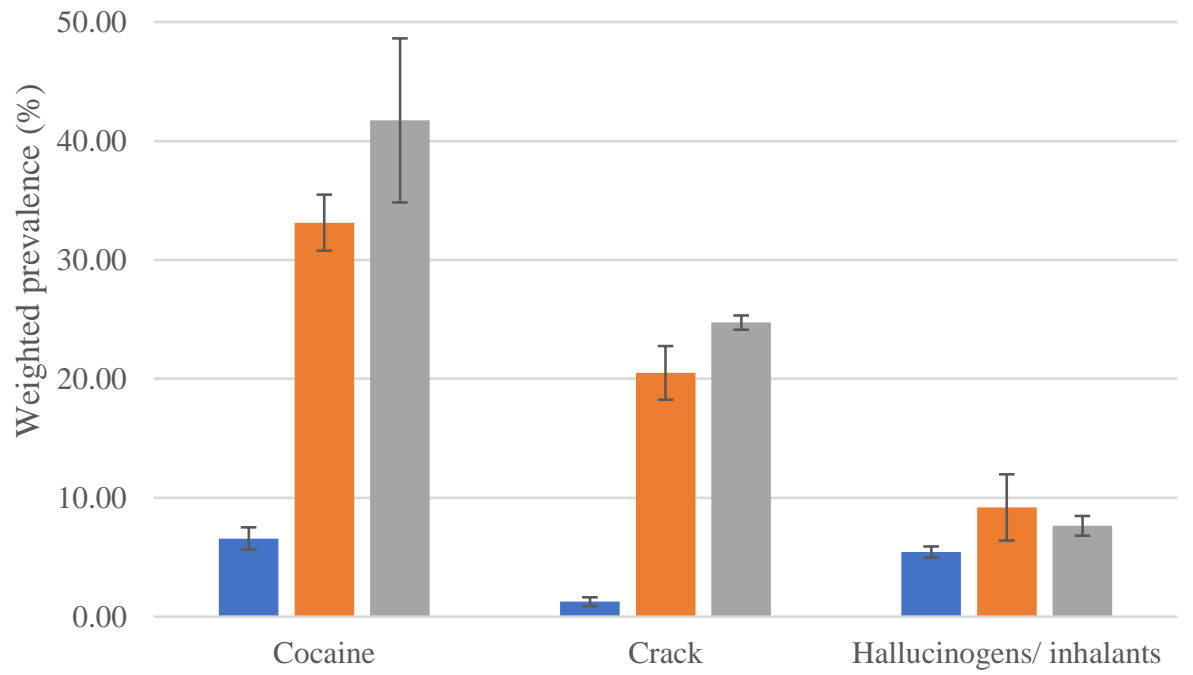

घ Past-month PPR only $\quad$ Past-month heroin only $\quad$ Past-month combination opioid 
APPENDIX C: List of Acronyms 
$\mathrm{aOR}$

APC

ASPE

AUD

CAPI

CARA

CBHSQ

CDC

CI

CUD

DAC

DEA

DSM

DUD

ED

GSL

HCC

HIV

HUD

ICD

IMF

LCA

LVMM
Adjusted odds ratio

Annual Percent Change

Assistant Secretary for Planning and Evaluation

Alcohol use disorder

Computer assisted personal interview

Comprehensive Addiction and Recovery Act

Center for Behavioral Health Statistics and Quality

Centers for Disease Control and Prevention

Confidence interval

Cannabis use disorder

The Drug Abuse Council

Drug Enforcement Agency

Diagnostic and Statistical Manual of Mental Disorders

Any drug use disorder

Emergency department

Good Samaritan Laws

Healthcare for Communities survey

Human immunodeficiency virus

Heroin use disorder

International Classification of Diseases

Illegally manufactured fentanyl

Latent class analysis

Latent variable mixture modeling 


\begin{tabular}{|c|c|}
\hline $\mathrm{m}$ & Slope \\
\hline MAT & Medication-assisted therapy \\
\hline MCL & Medical cannabis laws \\
\hline MCP & Medical cannabis program \\
\hline MMWR & Morbidity and Mortality Weekly Report \\
\hline MTF & Monitoring the Future \\
\hline NASPER & $\begin{array}{l}\text { National All Schedules Prescription Electronic Reporting } \\
\text { Act }\end{array}$ \\
\hline NESARC & $\begin{array}{l}\text { National Epidemiologic Survey on Alcohol and Related } \\
\text { Conditions }\end{array}$ \\
\hline NHIS & National Vital Statistics Survey \\
\hline NMUPO & Nonmedical use of prescription opioids \\
\hline NMUPS & Nonmedical use of prescription stimulants \\
\hline NPS & Novel psychoactive substances \\
\hline NSDUH & National Survey of Drug Use and Health \\
\hline NVSS & National Vital Statistics System \\
\hline ODUD & Other drug use disorder \\
\hline OPR & Opioid pain relievers \\
\hline OUD & Opioid use disorder \\
\hline PDAPP & Prescription Drug Abuse Prevention Plan \\
\hline PMDP & Prescription drug monitoring program \\
\hline PPR & Prescription pain relievers \\
\hline PR & Weighted prevalence; prevalence rate \\
\hline PWID & People who inject drugs \\
\hline
\end{tabular}




$\begin{array}{ll}\text { PWUO } & \text { People who use opioids } \\ \text { RTI } & \text { Research Triangle Institute } \\ \text { RX-OUD } & \text { Prescription opioid use disorder } \\ \text { SAMHSA } & \begin{array}{l}\text { Substance Abuse and Mental Health Services } \\ \text { Administration }\end{array} \\ \text { SE } & \text { Standard error } \\ \text { SG } & \text { Supplemental Graph } \\ \text { SID } & \text { State Inpatient Databases } \\ \text { ST } & \text { Supplemental Table } \\ \text { STRIDE } & \text { System to Retrieve Information from Drug Evidence } \\ \text { SUD } & \text { Substance use disorder } \\ \text { U.S. } & \text { United States } \\ \text { USD } & \text { United States dollars } \\ \text { USDHHS } & \text { Wide-ranging online data for epidemiologic research } \\ \text { WONDER } & \end{array}$




\title{
CURRICULUM VITAE
}

\author{
Diana Kuo Stojda \\ 5439 Pine Top Circle \\ Raleigh, NC 27612
}

(856) 520-0089 | diana.kuo@louisville.edu

\section{Education}

University of Louisville

Doctor of Philosophy, Epidemiology

The University of North Carolina - Chapel Hill

Bachelor of Science, Clinical Laboratory Science

Boston University

Master of Public Health, Epidemiology

University of Connecticut

Bachelor of Science, Pathobiology

Bachelor of Arts, German Studies
August 2015 - current

September 2010 - May 2012

September 2005 - January 2007

September 2001 - May 2005

\section{Teaching experience}

\section{University of Louisville - Louisville, KY}

Spring 2018

Fall 2017

Spring 2017
PHUN441 Epidemiology for Public Health (undergraduate course)

PHEP619 Biology of Disease (Masters-level course)

PHEP650 Advanced Topics: Epidemiology of Communicable Diseases (Masters-level course) 


\section{Presentations}

Stojda DK, Hollenbach R, Jones VF, Harris MJ, Klein JM, Owusu Kwarteng NA, Odoh C. (2019, March). Healthy Food Options Contributed by International Food Markets in Jefferson County, Kentucky. Poster presentation at the annual meeting of the American Academy of Health Behavior, Greenville, SC.

Stojda DK, Jackson T, Niyongabo D, Muvuka B, Harris M. (2018, November). Advocating for effective mining policies: A study of health effects of gold mining in Obuasi, Ghana. Oral presentation at the annual meeting of the American Public Health Association, San Diego, CA.

\section{Research experience}

University of Louisville - Health and Social Justice Scholar Project; Louisville, KY Principal Investigator, September 2017 - present

- Project title: Examining the Effects and Contributions of International Food Markets on Food Access in Jefferson County, Kentucky

- Designing a project assessing disparities related to food availability and barriers to access in Jefferson County, KY

- Responsible for submitting protocol and supplemental documents to Institutional Review Board

- Coordinating study activities and managing study team data collection and analyses responsibilities

- Engage local international food market businesses to understand how they contribute to the food ecosystem

- Collaborate with multiple stakeholders in Jefferson County, KY to recruit participants for individual interviews aimed at understanding barriers to food access in the Hispanic/Latino community

- Serve as a mentor to Master-level students by providing research feedback and technical training

University of Louisville - Department of Health Promotion and Health Behavior; Louisville, KY

Research Assistant, May 2017 - June 2017

- Project title: Health Effects of Gold Mining in Anyinam, Obuasi, Ghana

- Recruited and consented study participants in the village of Anyinam, Ghana

- Interviewed participants using a semi-structured questionnaire to determine the participant's perceived health and factors affecting their health

- Analyzed qualitative data with study team to ascertain themes related to their perceived health outcomes and causes 
University of Louisville - Department of Education Counseling and Psychology;

Louisville, KY

Research Assistant, January 2017 - present

- Project title: Families and Schools Together (FAST) for improving outcomes for children and their families (under review)

- Screened abstracts for studies to be included in this meta-analysis using specific eligibility criteria

- Retrieved literature and created reference trees for other studies

- Assisted in coding studies for eligible outcome measures and risk of bias

\section{University of Louisville - Department of Epidemiology and Population Health;}

Louisville, KY

Graduate Research Assistant, October 2015 - December 2017

- Project title: Coal Ash and Neurobehavioral Symptoms in Children Aged 6-14 Years Old

- Engaged in recruitment efforts, including in-person and mailings

- Assisted in participant consent/assent and neurotesting for child participants

- Set up in home air monitoring and filtering equipment

University of Louisville - School of Interdisciplinary and Graduate Studies, Louisville, KY

Graduate Research Assistant, February 2016 - August 2016

- Utilized SAS for data management functions and basic analyses

- Coordinated with various faculty and staff members of SIGS to correctly classify data

- Assisted in editing department web pages

- Trained to use SAS Visual Analytics to assist in creating reports for the University Provost

University of Louisville - Division of Infectious Diseases, Louisville, KY

Research Assistant, October 2015 - February 2016

- Project title: Streptococcus Pneumoniae Serotypes IN Adults 18 Years and Older with Radiographically-Confirmed Community-Acquired Pneumonia $(C A P)$ Interacted with patients, health care professionals, and insurance companies to retrieve and verify pneumonia vaccination history

- Worked collectively with other members of vaccine study team to ensure timely data collection

- Upheld all aspects of patient confidentiality and privacy 


\section{Other work experience}

Laboratory Corporation of America - Molecular Genetics; Research Triangle Park, $\mathrm{NC}$

Molecular Genetics Technologist, October 2014 - August 2015

- Conducted patient testing for various genetic mutations (e.g. Factor V, MTHFR) using a combination of PCR and gel electrophoresis technologies

- Provided training to technologists new to assays

- Evaluated and resulted high volume assays efficiently with minimal single repeat failures

- Ensured rapid turn-around-time by using multiple laboratory software systems

- Supported quality management efforts by completing daily and weekly 5S duties as well as providing input on improvement of methodology

\section{North Carolina Depart. of Agriculture \& Consumer Services, Rollins Laboratory;}

Raleigh, NC

Medical Laboratory Technologist II, December 2012 - October 2014

- Developed new procedures for testing for epidemiologic surveillance of reemerging zoonotic diseases

- Utilized advanced PCR technology with a high degree of accuracy and precision for veterinary diagnostic purposes while exceeding quality management expectations

- Acted as technologist-in-charge to facilitate training of new staff members and maintain workflow

- Constructed new appendices and forms to existing standard operating procedures to increase productivity

UNC Health Care - McLendon Laboratories; Chapel Hill, NC

Medical Technologist I, March 2012 - December 2012

- Worked independently and efficiently to perform a multitude of microbiological tests to ensure fast and correct results for patients without compromising medical ethics, in accordance with proper quality control measures

- Served as primary operator of testing not regularly run on the overnight weekend shift (e.g. PNA FISH)

- Acted as a liaison between laboratory management and healthcare staff by educating them on

China Care Foundation, Inc.; Westport, CT

Development Coordinator/Administrative Assistant, September 2007 - August 2010

- Improved constituent relationships by using multiple software to create donor reports/medical funding proposals, updating website content

- Completed activities essential for good office functionality

- Acted as primary in-house computer support employee

- Conducted research and advised on potential grants for the Foundation

- Reviewed and advised on suitability of applicants for volunteer and financial aid programs, as well as new China Care staff member 


\section{Skills}

- Technical: Microsoft Word, Excel, PowerPoint, Outlook, SAS, SPSS

- Languages: Fluent English and Taiwanese, conversational German

\section{Awards, Honors, and Certifications}

- Health and Social Justice Scholars - University of Louisville Health Science Campus, Fall 2016

- Out-of-State Merit Scholar Award - University of Louisville, Fall 2015

- Human Subjects Research Certification - Collaborative Institutional Training Initiative, Fall 2015

- $\operatorname{MLS}(\mathrm{ASCP})^{\mathrm{CM}}$ certification - American Society for Clinical Pathology, May 2012

- McClendon Laboratory Scholarship - UNC Health Care, August 2010

\section{Professional Affiliations}

- Society for Epidemiologic Research; January 2016 - current

- American Public Health Association; January 2016 - current

- American College of Epidemiology; January 2017 - current

- American Academy of Health Behavior; January 2018 - current 